\title{
Petrological Systematics of Mid-Ocean Ridge Basalts: Constraints on Melt Generation Beneath Ocean Ridges
}

\author{
CHARLES H. LANGMUIR ${ }^{1}$, EMILY M. KLEIN ${ }^{2}$, AND TERRY PLANK ${ }^{1}$
}

1. Lamont-Doherty Geological Observatory of Columbia University, Palisades, NY 10964

2. Department of Geology, Duke University, Durham, NC 27706

\begin{abstract}
Mid-ocean ridge basalts (MORB) are a consequence of pressure-release melting beneath ocean ridges, and contain much information concerning melt formation, melt migration and heterogeneity within the upper mantle. MORB major element chemical systematics can be divided into global and local aspects, once they have been corrected for low pressure fractionation and interlaboratory biases. Regional average compositions for ridges unaffected by hot spots ("normal" ridges) can be used to define the global correlations among normalized $\mathrm{Na}_{2} \mathrm{O}, \mathrm{FeO}, \mathrm{TiO}_{2}$ and $\mathrm{SiO}$ contents, $\mathrm{CaO} / \mathrm{Al}_{2} \mathrm{O}_{3}$ ratios, axial depth and crustal thickness. Back-arc basins show similar correlations, but are offset to lower $\mathrm{FeO}$ and $\mathrm{TiO}_{2}$ contents. Some hot spots, such as the Azores and Galapagos, disrupt the systematics of nearby ridges and have the opposite relationships between $\mathrm{FeO}, \mathrm{Na}_{2} \mathrm{O}$ and depth over distances of $1000 \mathrm{~km}$.

Local variations in basalt chemistry from slow- and fast-spreading ridges are distinct from one another. On slow-spreading ridges, correlations among the elements cross the global vector of variability at a high angle. On the fast-spreading East Pacific Rise (EPR), correlations among the elements are distinct from both global and slow-spreading compositional vectors, and involve two components of variation. Spreading rate does not control the global correlations, but influences the standard deviations of axial depth, crustal thickness, and $\mathrm{MgO}$ contents of basalts.

Global correlations are not found in very incompatible trace elements, even for samples far from hot spots. Moderately compatible trace elements for normal ridges, however, correlate with the major elements. Trace element systematics are significantly different for the EPR and the mid-Atlantic Ridge (MAR). Normal portions of the MAR are very depleted in REE, with litule variability; hot spots cause large long wavelength variations in REE abundances. Nomal EPR basalts are significantly more enriched than MAR basalts from normal ridges, and still more enriched basalts can erupt sporadically along the entire length of the EPR. This leads to very different histograms of distribution for the data sets as a whole, and a very different distribution of chemistry along strike for the two ridges. Despite these differences, the mean $\mathrm{Ce} / \mathrm{Sm}$ ratios from the two ridges are identical.

Existing methods for calculating the major element compositions of mantle melts [Klein and Langmuir, 1987; McKenzie and Bickle, 1988; Niu and Batiza, 1991] are critically examined. New quantitative methods for mantle melting and high pressure fractionation are developed to evaluate the chemical consequences of melting and fractionation processes and mantle heterogeneity. The new methods rely on new equations for partition coefficients for the major elements between mantle minerals and melts. The melting calculations can be used to investigate the chemical compositions produced by small extents of melting or high pressures of melting that cannot yet be determined experimentally. Application of the new models to the observations described above leads to two major conclusions: (1) The global correlations for normal ridges are caused by variations in mantle temperature, as suggested by Klein and Langmuir [1987] and not by mantle heterogeneity. (2) Local variations are caused by melting processes, but are not yet quantitatively accounted for. On slower spreading ridges, local variations are controlled by the melting regime in the mantle. On the EPR, local variations are predominantly controlled by ubiquitous, small scale heterogeneites. Volatile content may be an important and as yet undetermined factor in affecting the observed variations in major
\end{abstract} elements.

We propose a hypothesis, similar to one proposed by Allegre et al [1984] for isotopic data, to explain the differences between the Atlantic and Pacific local trends, and the trace element systematics of the two ocean basins, as consequences of spreading rate and a different distribution of enriched components from hot spots in the two ocean basins. In the Atlantic, the hot spot influence is in discrete areas, and produces clear depth and chemical anomalies. Ridge segments far from hot spots do not contain enriched

Mantle Flow and Melt Generation at Mid-Ocean Ridges

Geophysical Monograph 71

Copyright 1992 American Geophysical Union 
basalts. Melting processes associated with slow-spreading ridges vary substantially over short distances along strike and lead to the local trends discussed above, irrespective of hot spot influence. In the Pacific, enriched components appear to have been more thoroughly mixed into the mantle, leading to ubiquitous small scale heterogeneities. Melting processes do not vary appreciably along strike, so local chemical variations are dominated by the relative contribution of enriched component on short time and length scales. Thus the extent of mixing and distribution of enriched components influences strongly the contrasting local major element trends. Despite the difference in the distribution of enriched components, the mean compositions of each data set are equivalent. This suggests that the hot spot influence is similar in the two ocean basins, but its distribution in the upper mantle is different. . These contrasting relationships between hot spots and ridges may result from differences in both spreading rate and tectonic history. Unrecognized hot spots may play an important role in diverse aspects of EPR volcanism, and in the chemical systematics of the enupted basalts

The observations and successful models have consequences for melt formation and segregation. (1) The melting process must be closer to fractional melting than equilibrium melting. This result is in accord with inferences from abyssal peridotites [Johnson et al., 1990]. (2) Small melt fractions generated over a range of pressures must be extracted rapidly and efficiently from high pressures within the mantle without experiencing low pressure equilibration during ascent. This requires movement in large channels, and possibly more efficient extraction mechanisms than normally envisaged in porous flow models with small residual porosity. (3) Diverse melts from the melting regime produce variations in basalts that are observable at the surface. (4) Basalt data can be used to constrain the melting process (e.g. active vs. passive upwelling) and its relationship to segmentation. The data cannot be used to constrain the shape of the melting regime, however, for many shapes lead to similar chemical results. (5) Highly incompatible elements and U-series disequilibria results appear not yet to be explained by melting models, and may require additional processes not yet clearly envisaged.

\section{INTRODUCTION}

The basalts that form the upper portion of the ocean crust are the end product of melting of the mantle, migration of melt through the mantle towards the surface, and cooling and differentiation of magma near the surface. Because the basalts have experienced each of these processes, there is the possibility that they contain a chemical record of these events. Our challenge is to decipher their chemical codes to reveal their history.

Evidence to help us decipher the codes comes from observation, experiment, and theory. The observations show the chemical systematics that exist in mid-ocean ridge basalts (MORB), and the relationships between the chemical compositions and physical aspects of the ocean floor such as axial depth, crustal thickness and tectonic setting. Experiments provide the necessary data to model chemical changes that occur under different conditions of temperature, pressure and composition. Theory aids in imagining what processes could occur that cause chemical changes in basalts, and is the sole means of relating the observations and experiments to quantitative models of the entire crust formation process.

Currently, three major factors are considered to contribute to the diversity of MORB compositions. (1) Crystallization and differentiation processes at shallow levels in the crust and uppermost mantle modify the compositions of mantle melts. (2) Melting itself can lead to differences in composition, depending on the temperatures and pressures involved, and the modes of melt migration and segregation. (3) The mantle source may $v$ ary in its major element, crace element and isotopic composition, which can lead to inherited variations in basalt compositions.

Recognizing and isolating the different effects of melting and crystallization on the compositions of erupted basalts is a fundamental and ongoing problem in igneous petrology.
Historically, the debate centered on whether basalt compositions are close to those of actual melts of the mantle [Green and Ringwood, 1967; Green, 1971; Gast, 1968], or whether their compositions have been so modified by differentiation in crustal magma chambers [O'Hara, 1965; $\left.O^{\prime} H a r a, 1977\right]$ or complex melting processes [O'Hara, 1985] that their melting histories are completely obscured. Although O'Hara's original argument that most basalts lie on lowpressure cotectics and thus are not "primary" is correct, it is now generally accepted that basalts still preserve aspects of their mantle melting history. The question of the relative importance of melting and differentiation processes is still not fully resolved, however, as will be clear from later discussion in this paper. Certainly one of the major questions is how well simple models of melting and differentiation approximate the more complex natural systems [e.g., O'Hara, 1977, 1985; Allegre and Minster, 1978; Richter, 1986; Plank and Langmuir, 1992].

It also has long been recognized that the mantle must be chemically heterogeneous on various scales. For example, hot spots are associated with large-scale enrichments of incompatible trace elements, such as $\mathrm{K}$ and $\mathrm{Ba}$, and radiogenic isotope ratios, such as ${ }^{87} \mathrm{Sr} /{ }^{86} \mathrm{Sr}$ [e.g., Schilling, 1975; Verma and Schilling, 1982; Schilling et al., 1983], compared to ocean ridges of average depth. The isotopic enrichments, in particular, require isolation of a mantle reservoir with enriched parent/daughter ratios for substantial portions of Earth history. On a smaller scale, there is isotopic variability in single seamounts [Zindler et al., 1984] and over short time intervals for an individual ridge segment [e.g., Shirey et al., 1987], and heterogeneities on the scale of meters are observed in ultramafic massifs such as the Ronda peridotite [Reisberg and Zindler, 1986/87). Although it is more difficult to distinguish major element source heterogeneity from the overprinting effects of melting and crystallization, some of the major 
element variability in MORB has been ascribed to major element variations in the mantle beneath ridges [e.g., Langmuir and Hanson, 1980; Sigurdsson, 1981; Bryan and Dick, 1982; Klein and Langmuir, 1987, hereafter referred to as $K L 87]$.

Thus, the effects of source composition, melting and crystallization form a complex web of chemical signatures. Our task is to understand what aspects of the chemical signature tell us about each process. Only then can one investigate the implications of basalt chemistry concerning melt formation and segregation.

We believe that developments in petrology in the past several years allow a much clearer separation among the effects of source composition, melting and crystallization, although there are still areas of dispute. Such developments include improvements in quantitative modeling of igneous processes, the acquisition of high-quality chemical data for basalts from diverse ridge segments, and more detailed experimental studies on peridotite melting and melt differentiation. It is now possible to better constrain those aspects of the data that may be attributed to each process, and to identify more clearly those aspects of the data for which interpretation remains ambiguous.

Our approach in this paper is first to present a working model for overall variability in MORB, which serves as a framework for subsequent presentation and discussion. We then present some basic concepts as necessary vocabulary. Some of this material is quite elementary, but is included to provide the necessary background to make these notes more accessible to non-petrologists. We then present observations of the major element chemistry of MORB, emphasizing aspects of the data that are critical to the problems of melting and melt migration. The basic major element observations are used to develop specific questions concerning their origin. We then consider modeling techniques, and present a new quantitative model for major elements that is able to treat fractional melting and mantle heterogeneity quantitatively. These modeling techniques are then applied to the questions raised by the data. Finally, we examine trace element data to test how they correspond to the major element models, and what new light they throw on the issues of melt formation and migration.

There are other important petrological approaches that we do not include in this paper. Notably, recent work on abyssal peridotites [Dick et al., 1984; Michael and Bonatti, 1985; Johnson et al., 1990; Johnson and Dick, 1992] has provided important evidence concerning melt formation and segregation, and has been able to address from a different point of view many of the same problems discussed in this paper. The abyssal peridotite literature is an important and necessary scientific complement to the work on basalts that is discussed here.

This paper includes a significant amount of background and review material for the non-specialist, in an effort to make the reasoning clear. However, it also includes new observations of the systematics of MORB compositions, new developments in modeling mantle melting and crystallization at high pressure, new constraints on the nature of mantle melting and crystallization at high pressure, new constraints on mantle melting chemistry and processes, and a new model for the origin of the local systematics of ocean ridges and the differences between MORB erupted on the Mid-Atlantic Ridge and East Pacific Rise.

\subsection{A Working Model}

It will help to understand the relevance of much of the material that follows if it can be placed in an overall framework for ocean crust creation.

It has been understood for many years that the ocean crust is formed by melting of the mantle as a result of decompression during upwelling beneath ocean ridges [e.g., Carmichael et al., 1974; Oxburgh, 1980 and references therein]. Mantle ascending beneath the ridge will follow an adiabatic gradient of $1^{\circ}$ to $2^{\circ} \mathrm{C}$ of temperature drop for each kilobar of ascent. The mantle solidus that controls where melting begins has a very different temperature gradient of about $13^{\circ} / \mathrm{kbar}$, so that ascending mantle inevitably intersects the solidus and begins to melt (Figure 1). The pressure at which such melting begins is controlled by the overall temperature of the mantle: hotter mantle intersects the solidus deeper than cooler mantle [e.g., Schilling, 1973].

The breaking of crystal bonds that occurs during melting requires heat, and this heat of fusion is provided internally by the portion of mantle that is ascending. This causes the mantle to cool as it melts, and therefore its temperature decreases with decreasing pressure more rapidly than the adiabatic gradient (Figure 1). Because the energy available for melting depends on the temperature difference between the melting adiabat and the solidus at a given pressure, the more the parcel of mantle ascends, the more it melts. The amount of melting that results is about $1-2 \%$ per kbar of pressure release.

These considerations need to be combined with the overall geometry of the melting regime beneath ridges. Various possible geometries were discussed by Oxburgh [1980], but the triangular shape with a flat bottom that results from adiabatic upwelling of mantle with a laterally uniform temperature is now most accepted (see McKenzie and Bickle, [1988] for a useful discussion; Figure 2). The amount of melt in the melting regime is controlled by the amount of pressure release that each individual parcel of mantle within the melting regime has undergone (Figure 2). The higher the mantle temperature, the more melt that is produced, and hence the greater the thickness of the oceanic crust [e.g., McKenzie, 1984].

Because the melt is less dense than the mantle, it will move as the mantle melts. Therefore the many different melts present at any one time within the melting regime may erupt independently, mix, or react with the mantle through which they pass, in a collection of processes that have been called "dynamic melting" [Langmuir et al., 1977]. The mean composition of the ocean crust, however, will be the mean composition of melts pooled from the melting regime.

The mean melt composition, and its relationship to mantle temperature and crustal thickness, can be envisioned simply by considering the "residual mantle column" that emerges outside the melting regime for each increment of spreading (Figure 2). 


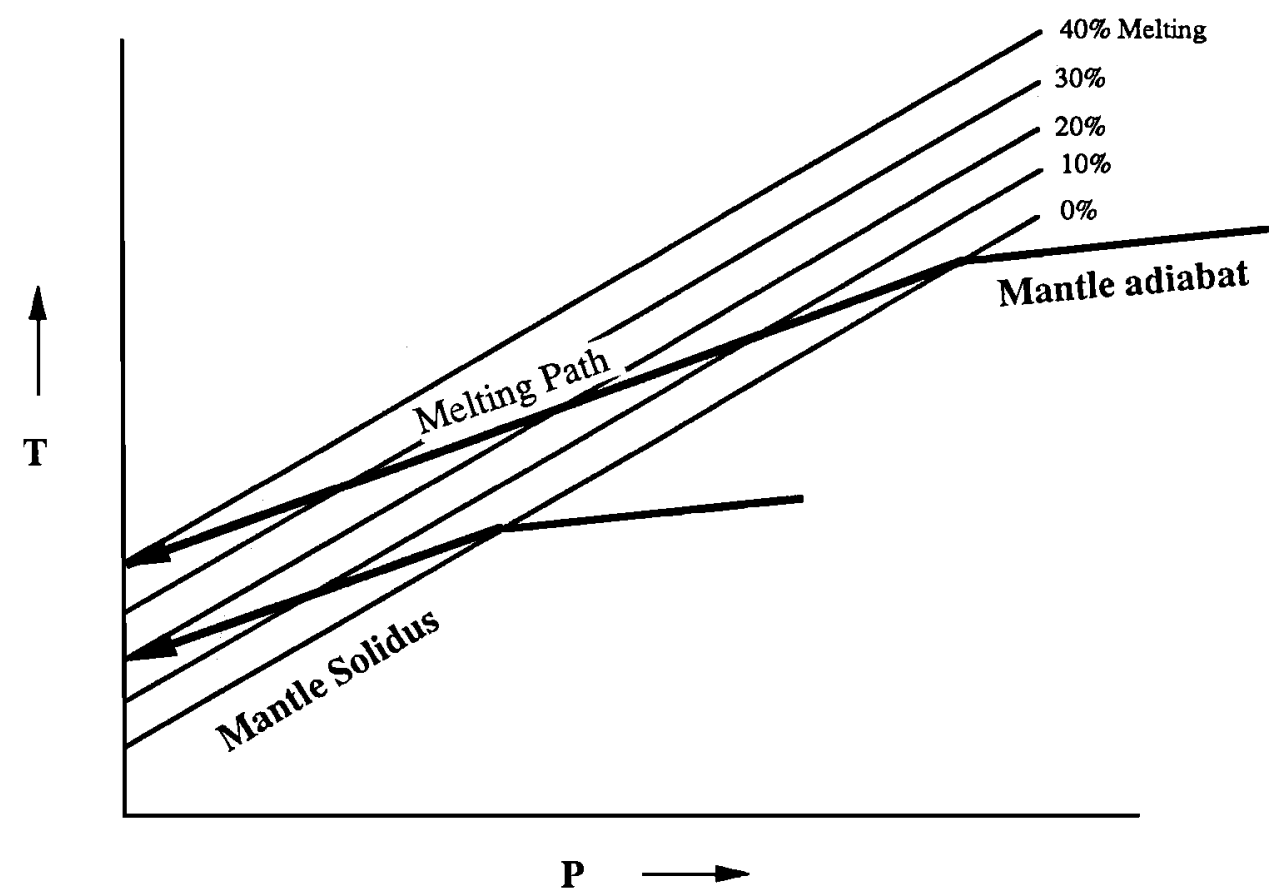

Fig. 1. Schematic pressure $(P)$ vs. temperature $(T)$ diagram showing melting paths for adiabatically ascending mantle. Melting contours are evenly spaced for illustration only. The two paths are for two different mantle temperatures. The further a mantle parcel ascends, the greater the extent of melting.

This column reflects all that has happened within the melting regime [Plank and Langmuir, 1992], and therefore provides a one dimensional summary of the melting regime. The deeper portions of the residual mantle column are produced at the outer corner of the melting regime, while the shallowest are produced at the center of the melting regime. The residual mantle column shows increasing depletion in melt upwards in the column, reflecting the increasing extent of melting upwards in the melting regime. The mean melt reflects the pooling together of all the various melts that were extracted to form the column. The mean pressure reflects the average pressure from which the melts are derived. Crustal thickness is the total amount of melt that is extracted, which is simply the mean extent of melting times the column height, if extraction is complete.

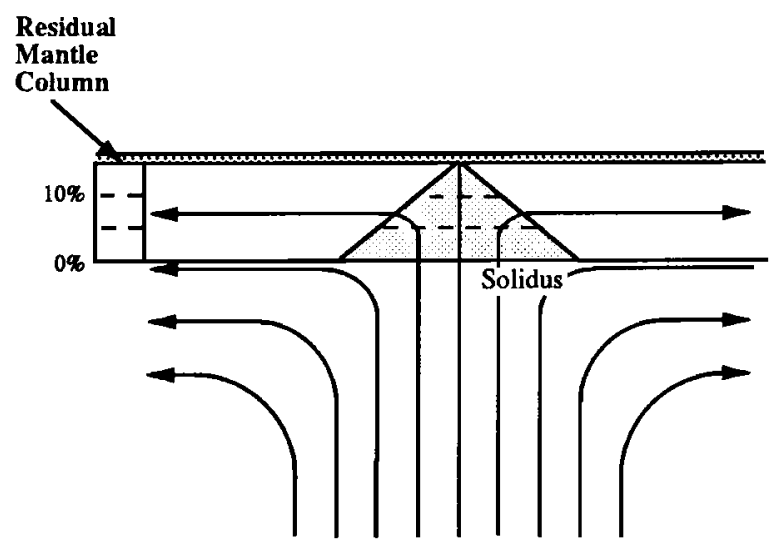

Cold Mantle

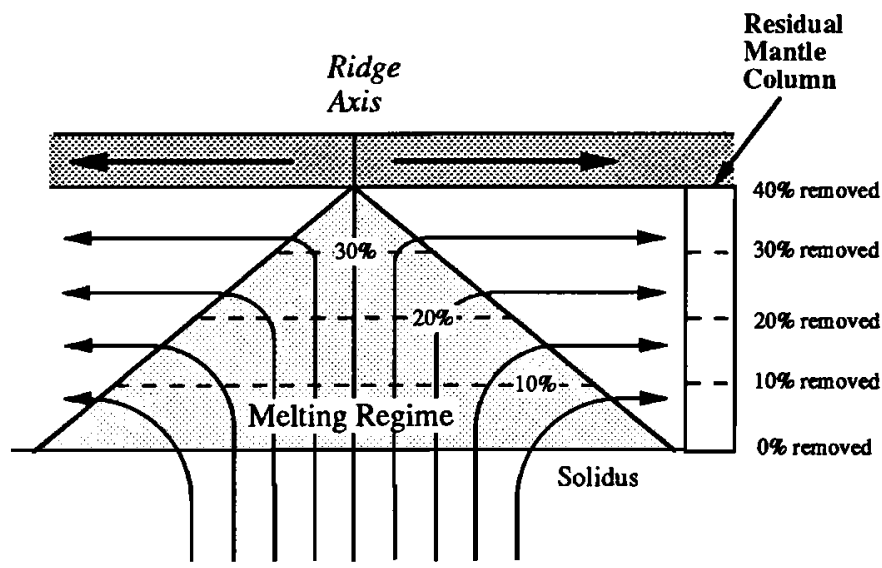

Hot Mantle

Fig. 2. Idealized steady-state melting regimes produced by spreading of the plates [after Plank and Langmuir, 1992]. Solid curves with arrows are mantle flow paths through the melting regime. Dashed lines are contours of the extent of melting in the melting regime or the extent of melt removed in the residual mantle column (RMC). The two melting regimes are for two mantle temperatures. Hotter mantle intersects the solidus deeper (see Figure 1), leading to greater extents of melting, a taller RMC, and thicker crust. 
These considerations can be combined with simple chemical considerations for a general model of the composition and thickness of the ocean crust in response to lateral variations in mantle temperature [KL87]. The chemical considerations are that some elements, such as $\mathrm{Na}_{2} \mathrm{O}$, strongly prefer the melt to the solid mantle, and hence their concentrations in mantle melts are roughly inversely proportional to the extent of melting [Jaques and Green, 1980; Dick et al., 1984]. Other elements, such as $\mathrm{FeO}$, are sensitive to the pressure of melting, with higher pressures leading to higher $\mathrm{FeO}$ contents in the melt [Langmuir and Hanson, 1980]. The eritical observation from regionally averaged MORB is that the extent of melting (inferred from $\mathrm{Na}_{2} \mathrm{O}$ ) and the pressure of melting (inferred from $\mathrm{FeO})$ correlate positively [KL87]. This requires a model where higher mean extents of melting occur at higher mean pressures. To accomplish this, extraction must be very efficient, with melts from deep in the melting regime migrating toward the surface without re-equilibrating at shallow levels. Then higher mantle temperatures lead to taller melting columns, greater mean extents and pressures of melting, and greater crustal thickness. Ocean ridge depth is controlled largely by three factors: crustal thickness, and the temperature and composition of the underlying mantle. All of these factors lead to shallower depths for hotter mantle. Therefore relationships among crustal composition, crustal thickness and ridge depth can be envisioned as a response to variations in mantle temperature along the ocean ridge system.

At the same time, consideration of the melting process above indicates the likelihood of substantial compositional variability within the melting regime. Within any single melting regime created by a given mantle temperature, the smallest degree melts occur at the greaiest pressures. This could lead to the opposite relationship between the extent of melting and pressure of melting for diverse melts from a single melting regime, as compared to average melts from diverse melting regimes created by variations in mantle temperature. Therefore, chemical variations can occur within a single melting regime that lead to diverse melts of the mantle in local regions. These chemical variations can have the opposite relationship between pressure and temperature of melting than that observed for mean melts among different melting regimes. Such variations could lead to local compositional variations that are distinct from global compositional variations.

The melts of the mantle do not arrive at the surface in an unadulterated state. They may pass through colder lithosphere, or react or undergo fractionation at high pressure. They certainly pass through magma fractionation systems at lower pressures, just beneath or within the crust, where substantial differentiation of their compositions may take place by a variety of processes. The simplest form of differentiation occurs in a closed system at low pressure, which we will refer to as "low pressure differentiation." All other processes of differentiation, such as high-pressure or in situ fractionation, we refer to as "complex differentiation processes."

This working model provides a qualitative picture of ocean crust formation: lateral variations in mantle temperature control the mean melt composition; variations in melt composition within a single melting regime control local chemical variability; and differentiation processes modify everything. We can see from the model the difficulty and complexity of addressing many important questions concerning melt formation and segregation. The model does not address the details of the melting process, such as whether melting occurs by fractional or equilibrium melting, or to what extent extraction is efficient. It assumes a homogeneous mantle when we know the mantle is heterogeneous in many aspects of its composition. It is a two-dimensional, acrossstrike model, when much of the excitement in the study of ridges in the past decade has concerned along-strike variations, and the next decade will reveal new discoveries about changes through time. It assumes passive upwelling when upwelling in some regions may be actively buoyant. It is spreading rateindependent when spreading rate has long been considered to be a primary control on the variability of ocean ridges.

The model serves, however, as a useful mental framework to aid in the digestion of what is presented on the following pages. It provides a structure within which to view the great complexity and variability of MORB, while the data from MORB can in turn be used to test the overall validity of the model. As we will see, the model requires elaboration and modification in order to account for aspects of the data that probably result from the complexities mentioned above.

\section{BASIC THEORETICAL CONCEPTS}

\subsection{Phase Diagrams}

The classical approach to petrologic problems is through phase diagrams. In a simple ternary system consisting of phases $\mathrm{A}, \mathrm{B}$ and $\mathrm{C}$ (Figure $3 a$ ), the major element composition of a liquid produced by partial melting of the three-phase solid assemblage will be the eutectic composition, regardless of the initial phase proportions. The eutectic melt is invariant and conveys no information concerning source composition or extent of melting. Many experimental studies, in the interests of thermodynamic rigor and in the traditicn of Bowen, have concentrated on such simple systems (e.g., the $\mathrm{CaO}-\mathrm{MgO}$ $\mathrm{Al}_{2} \mathrm{O}_{3}-\mathrm{SiO}_{2}$ or CMAS system). If the $\epsilon$ arth were like these systems, and the magmas were on low-pressure cotectics, then the major element composition of the magma would contain no information about extent of melting or source composition.

Simple phase diagrams have provided the framework for petrologic studies throughout most of the century; they are the basis for how petrology is taught, and how petrologists think about petrologic problems. Because it is difficult to graphically represent even four components in a phase diagram, an aim of many petrologic studies in recent years has been to reduce the multi-component mantle system to a more tractable three or four component system [e.g., Stolper, 1980; Elthon and Scarfe, 1984]. An example of such a "pseudoternary" phase diagram is shown in Figure $3 b$. Pseudoinvariant points and pseudo-invariant curves on these diagrams are really regions in multi-component space [Elthon and Scarfe, 1984; Stolper, 1980; Presnall and Hoover, 1984; Fujii 


\section{a. Ternary Phase Diagram}

$\mathbf{A}$

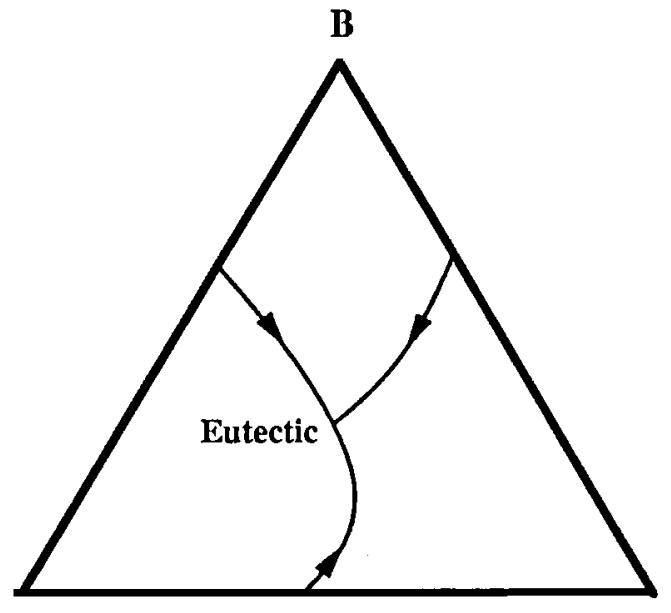

b. Pseudo-Ternary Phase Diagram

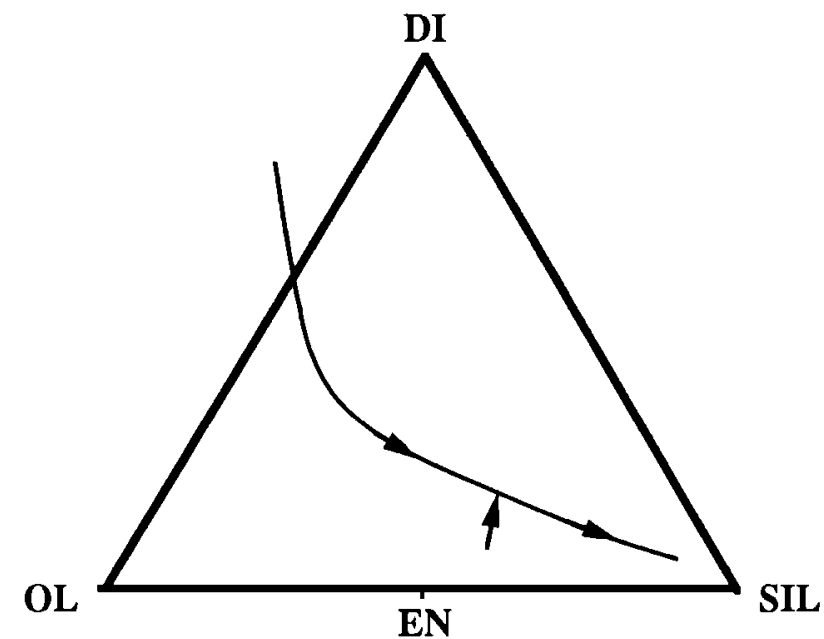

Fig. 3. a) A simple temary phase diagram with a eutectic. Any bulk composition within the triangle would yield an initial melt composition at the temary eutectic. b) Example of a pseudo-ternary phase diagram [after Stolper, 1980].

and Scarfe, 1985]. On such pseudo-ternary diagrams, however, one is naturally drawn to think of true invariant points, and by extension, one might conclude that mantle melts reflect neither source composition nor extent of melting. Indeed, earlier studies described mantle melting as "eutectic-like" [e.g., Yoder, 1976; Mysen and Kushiro, 1977], and many later studies have interpreted these diagrams as if melting were eutectic-like.

As many have recognized, however, there are three fundamental differences between melting in simple systems and in the mantle. First, there are eight important components (Si$\mathrm{Al}-\mathrm{Fe}-\mathrm{Mg}-\mathrm{Ca}-\mathrm{Na}-\mathrm{Ti}-\mathrm{Cr}$ ) and additional minor components (e.g., $\mathrm{K}, \mathrm{Fe}^{3+}, \mathrm{P}, \mathrm{CO}_{2}$, and $\mathrm{H}_{2} \mathrm{O}$ ) in the real system. For a given number of mineral and melt phases, the number of components governs the degrees of freedom: the large number of components and limited number of phase $i$ involved in mantle melting precludes invariant melting, which means that the melt composition can vary as melting proceeds. Second, all mantle phases show solid solution; for example, the $\mathrm{Mg} / \mathrm{Fe}$ ratio of liquids in equilibrium with olivine increases as melting proceeds. Third, the mantle melts polybarically, so the constant pressure restriction of most phase diagrams does not hold. These theoretical considerations have been conclusively verified by recent experimental studies that show a wide range of melt compositions in equilibrium with lherzolite for a single bulk composition and a single pressure [e.g., Jaques and Green, 1980; Fujii and Scarfe, 1985; Takahashi and Kushiro, 1983; Falloon and Green, 1988; Falloon et al., 1988]. It is nonetheless tempting to draw a pseudo-ternary diagram and use it in a classical manner to discuss mantle melting. Therefore, it is important to emphasize that:

1. There are no mantle eutectics, even on pseudo-temary diagrams, and therefore mantle melting produces a range of compositions;

2. Melting is a polybaric process, and melts form and probably segregate over a range of pressures.

Phase diagrams provide a convenient graphical representation of complex compositions; they often can accurately predict what phases are stable; and they are the conventional vocabulary of petrologists. They can be used to illustrate principles and to demonstrate in a straightforward way the effects of complex processes. They can also be used with care to consider complex equilibria. These aspects make them a useful tool, as illustrated by othe: papers in this volume [Hess, this volume; Grove et al., this volume]. For our purposes, however, the complexity and texture of the chemical signal is often reduced after projecting onto a pseudo-ternary diagram, and thus some of the information contained in the signal is lost. More subtly, the use of over-simplified phase diagrams can lead one who is not aware of the full complexity to think of multi-component equilibria incorrectly. For these reasons, in this paper we do not emphasize the phase diagram presentation of equilibria, and rely instead on the quantitative calculation of equilibria using individual elements or components.

\subsection{Partition Coefficients}

Almost all the chemical variability we will discuss reflects the fractionation of elements that occurs during the separation of liquid and crystal. A very simple concept that describes this fractionation is the partition coefficient, $K_{d}$, defined for a given single element or component as follows:

$$
\mathrm{K}_{\mathrm{d}}=\mathrm{C}_{\mathrm{s}} / \mathrm{C}_{\mathrm{L}}
$$

where $C_{s}$ is the concentration of an element in a particular mineral, and $C_{L}$ the concentration of the element in the liquid. 
For a multiphase solid assemblage the $K_{d}$ 's for each individual mineral are combined into a "bulk distribution coefficient" (D) which is simply the concentration in the total solid $\left(C_{s}\right)$ divided by the concentration in the liquid. Hence:

$$
\mathrm{D}=\mathrm{C}_{\mathrm{S}} / \mathrm{C}_{\mathrm{L}}
$$

$=($ fraction mineral 1$) *\left(\mathrm{~K}_{\mathrm{d}}\right.$ for mineral 1$)+($ fraction mineral $2) *\left(K_{d}\right.$ for mineral 2$)+$ etc.

$$
=\Sigma X_{j} K_{d}^{j}
$$

where $X$ is the fraction of the mineral and $K_{d}$ is the $K_{d}$ for element $i$ between phase $j$ and the liquid. (Symbols used throughout the paper are given in Table 1.)

The $K_{d}$ is actually a synthesis of a great deal of thermodynamic information. Determining $K_{d} s$ for real, nonideal systems from thermodynamic data on pure components involves complex, empirical solution models. However, the $\mathrm{K}_{\mathrm{d}}$ contains this information in a single value, and can be measured directly from experimental or natural mineral-melt pairs. Thus, if $\mathbf{K}_{d}$ 's can be parameterized as a function of temperature, pressure, and composition, a thermodynamically valid and relatively simple description of equilibrium can result [Langmuir and Hanson, 1981; Nielsen and Dungan, 1983]. We will use this $K_{d}$ approach to calculate both crystallization and melting. It is important to note that temperature, pressure and compositional variations affect the absolute value of the partition coefficient, as illustrated for the olivine $\mathrm{K}_{\mathrm{dM}_{\mathrm{B}}}$ in Appendix B. Careful attention to the influence of these variables is particularly important for quartitative modeling of major element variations using a $K_{d}$ approach, as shown in subsequent sections.

Another advantage of the $K_{d}$ approach is that it unifies the treatment of major and trace elements. Trace elements have long been modeled with partition coefficients [Gast, 1968; Shaw, 1970; Schilling, 1971]. Elements with partition coefficients $<1$ are concentrated into the liquid and thus are "incompatible" in the solid phases. With respect to mantle minerals, such incompatible elements include the heavy alkali elements, the light rare earth elements, $\mathrm{Ba}, \mathrm{Th}, \mathrm{U}, \mathrm{Ta}, \mathrm{Nb}$ and $\mathrm{Pb}$. Some major elements, such as $\mathrm{Na}$ and $\mathrm{Ti}$, have small $\mathrm{K}_{\mathrm{d}}$ 's for most mantle minerals, and hence behave incompatibly. Elements that are preferentially incorporated into a particular mineral are "compatible" with respect to that mineral. For example, $\mathrm{Sr}$ is preferentially incorporated into plagioclase; it is thus incompatible if plagioclase is absent, but becomes compatible if plagioclase forms a significant fraction of the total solid assemblage. Trace elements can be more or less compatible than major elements. For exarr.ple, $\mathrm{Fe}, \mathrm{Mg}$ and $\mathrm{Ni}$ are all compatible in olivine, but $\mathrm{Ni}$ is the most compatible. Thus, the same principles can be applied to both major and trace elements, and each element becomes a part of a single spectrum of variability when viewed from the perspective of their $\mathbf{K}_{d}$ 's. This perspective is exemplified by the use of "Onuma diagrams" [e.g., Onuma et al., 1968; Philpotts, 1978] which show that different elements that occupy the same site in a mineral have partition coefficients that vary smoothly as a function of atomic radius, independent of their concentration in the liquid.

This idea, that each element is part of a continuum of variability, has been put to great use in the interpretation of trace element variations, and in particular in the interpretation of the rare earth elements (REE). The REE vary progressively in the extent of their incompatibility with atomic number, because all of them have a similar outer electron shell, and therefore the change in their chemical behavior results principally from the progressive change in ionic radius with atomic number. With respect to the primary mantle minerals, the light rare earth elements (LREE), such as La or Ce, are most incompatible, while the heavy rare earth elements (HREE), such as $\mathrm{Yb}$ or $\mathrm{Lu}$, are only moderately incompatible and, if garnet is present, may become compatible. In order to make use of this regular variation in compatibility, it is common to plot the REE abundances in rocks as a function of increasing atomic number (called a Coryell-Masuda diagram) and normalize these abundances to a standard, such as chondritic meteorites, to eliminate the natural saw-toothed abundance pattern that results from the preferential creation of even numbered nuclides during nucleosynthesis. The resulting REE pattern can often reveal, at a glance, important features of the petrogenesis of the rock.

\subsection{Mass Balance and Simple Equations for Melting and Crystallization}

The simplest constraint for both major and trace elements is mass balance between solid and liquid:

$$
\mathrm{C}_{\mathrm{o}}=\left(\mathrm{F} * \mathrm{C}_{\mathrm{L}}\right)+\left[(1-\mathrm{F}) * \mathrm{C}_{\mathrm{S}}\right]
$$

where $C_{0}$ is the initial concentration of the element in the system, often called the "bulk composition", $F$ is the fraction of liquid, and as noted above, $\mathrm{C}_{\mathrm{S}}$ is the concentration of the element in the total solid assemblage. Mass balance applies strictly to closed systems, although there are mass balance constraints even in open systems.

It is useful to combine the simple concept of a distribution coefficient with mass balance. By sutstituting equation (2) into equation (4) we arrive at:

$$
\mathrm{C}_{\mathrm{L}} / \mathrm{C}_{\mathrm{o}}=1 /[\mathrm{F}+\mathrm{D} *(1-\mathrm{F})]
$$

This simple equation was developed by Schilling [1966] and Gast [1968], and has formed the basis for most trace element modeling. It is called the "batch melting" or "equilibrium melting" equation when applied to melting, and the "equilibrium crystallization" equation when applied to crystallization.

Although this equation is simple, it is also general and powerful. First, it applies to all elements, major elements as well as trace elements. Second, provided there is enough time for liquid and solid to reach equilibrium, equation (5) describes any point in the melting or crystallization process. Phases may disappear and melt may move, but at any given point in time the concentrations in the solid and liquid define the distribution coefficient, and thus equation (5) holds. 
TABLE 1. Definitions \& Acronyms

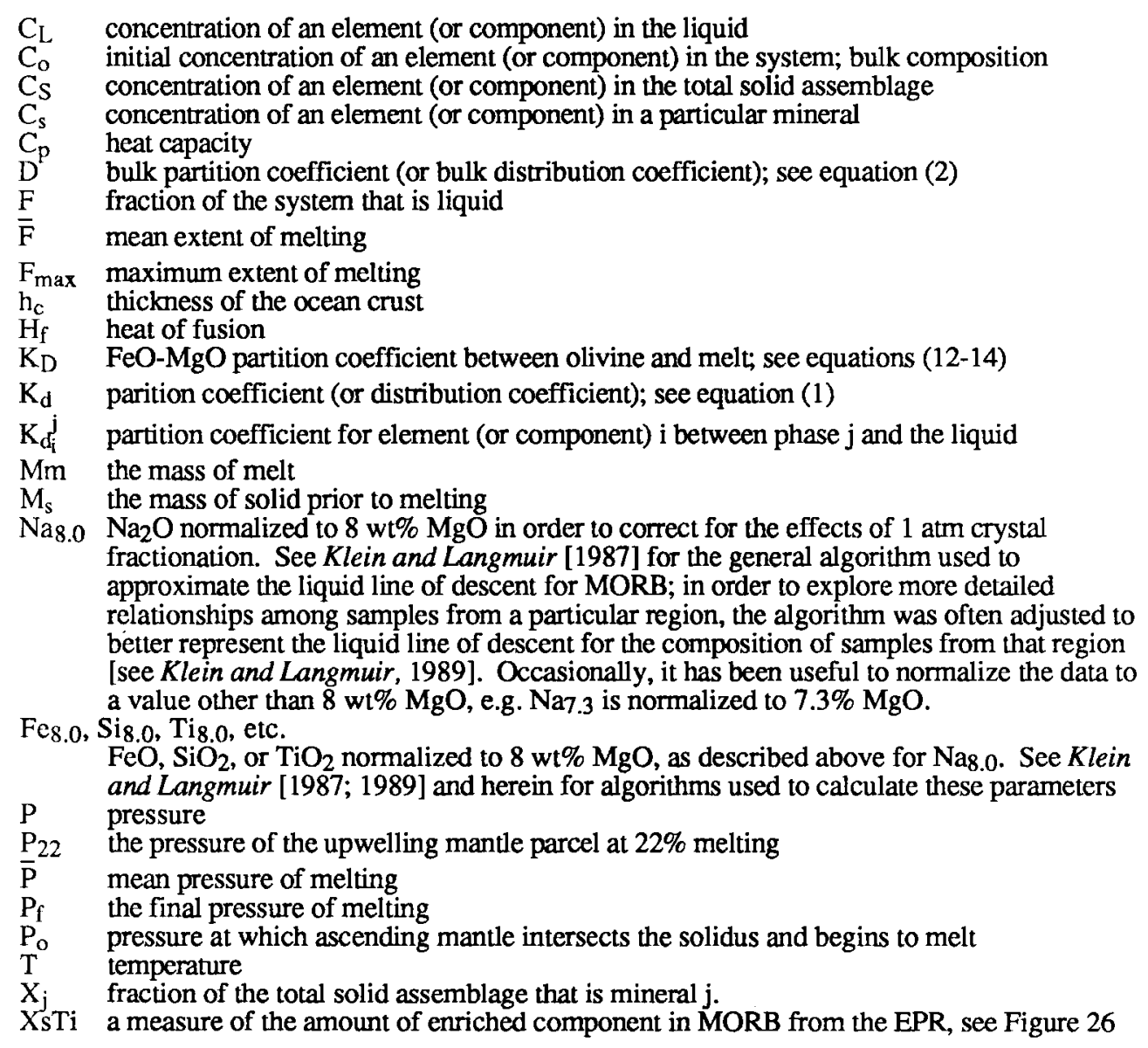

$\begin{array}{ll}\text { AFM } & \text { Accumulated fractional melting } \\ \text { AMAR } & \text { Alvin at the MAR, region near } 36^{\circ} \mathrm{N} \text { on the MAR } \\ \text { EPR } & \text { East Pacific Rise } \\ \text { FAMOUS } & \text { French American Mid-Ocean Undersea Study, } \\ & \text { a region near } 36^{\circ} 30^{\prime} \mathrm{N} \text { on the MAR } \\ \text { JDF } & \text { Juan De Fuca Ridge } \\ \text { LLD } & \text { Liquid lines of descent } \\ \text { LREE } & \text { Light rare Earth elements } \\ \text { MAR } & \text { Mid-Atlantic Ridge } \\ \text { Mg\# } & \text { Mg/(Mg+Fe), atomic ratio } \\ \text { MORB } & \text { Mid-ocean ridge basalt(s) } \\ \text { N-MORB } & \text { Normal mid-ocean ridge basalt } \\ \text { E-MORB } & \text { Enriched mid-ocean ridge basalt } \\ \text { T-MORB } & \text { Transitional mid-ocean ridge basalt } \\ \text { OFZ } & \text { Oceanographer Fracture Zone } \\ \text { PRMC } & \text { Periodically replenished magma chambers } \\ \text { REE } & \text { Rare earth elements } \\ \text { HREE } & \text { Heavy rare earth elements } \\ \text { LREE } & \text { Light rare earth elements } \\ \text { RMC } & \text { Residual mantle column }\end{array}$


The simple mass balance equation illustrates several important principles concerning the relative effects of melt fraction and partition coefficient on liquid composition. Figure $4 a$ shows the concentrations in the melt of elements with different D's as a function of $F$ for batch melting. Note first that as $F$ goes to 1 (all melt), $C_{L} / C_{o}$ of all elements converge on 1 , the initial starting concentration. Highly incompatible elements $(D \sim 0)$ vary as $1 / F$, and thus can be sensitive indicators of the extent of melting (for a given $C_{0}$ ) or crystallization. For all values of $F$, compatible elements $(D>$ 1) are preferentially partitioned into the solid, and therefore relative to the source concentration are in lower abundance in the melt. As $\mathrm{F}$ becomes smaller than $\mathrm{D}$, the concentration of all elements approaches 1/D. This is true even for highly incompatible elements as $F$ approaches 0 . Thus, for incompatible elements, the maximum enrichment that can be achieved is $1 / \mathrm{D}$, and the most any two elements in the liquid can be fractionated from one another is the ratio of their D's (Figure $4 b$ ). This $1 / \mathrm{D}$ constraint applies to a multitude of melting models, and thus is a very useful relationship.

Equation (5) and Figures $4 a$ and $4 b$ apply to both equilibrium melting and equilibrium crystallization. For melting the system begins as total solid ( $F=0)$, whereas for crystallization the system begins as total melt $(F=1)$. The greatest incompatible element enrichments and fractionations occur at small values of $F$, and change little at large values of $F$. Compatible elements, on the other hand, change most at large values of $F$ and are constrained by the $1 / \mathrm{D}$ limit over a wide range of $F$ as $F$ gets small. Thus small extents of partial melting affect incompatible elements substantially, but compatible elements less, while small amounts of crystallization affect compatible elements substantially, but incompatible elements and particularly incompatible element ratios (Figure $4 b$ ) very little. For these reasons, variations in the ratio of two incompatible elements are a more useful indicator of processes other than crystallization, such as variability in the extent of melting or in the source composition.

More complex processes affect these co:clusions to a certain extent. For example, if the phases enter the melt in different proportions than they are present in the solid, then the D for any element changes as a function of $F$. This leads to:

$$
\left.C_{L} / C_{o}=1 /\left[F^{*}(1-p)+D_{o}\right)\right]
$$

where $D_{0}$ is the bulk partition coefficient when $F$ is zero [calculated using equation (3)], and $p$ is a distribution coefficient weighted according to the phase proportions entering the liquid (calculated using an equation similar to equation (3) except that $X_{j}$ would refer to the fraction of each mineral entering the melt). The difference in results using equation (6) as compared to equation (5) becomes apparent for elements that have high partition coefficients in the minerals that are melting preferentially (i.e., they have p's approaching or exceeding 1). For incompatible elements (both $D_{0}$ and $p$ near 0 ), there is little difference. Equation (6) rather than equation (5) is more applicable to mantle melting, since aluminous phases and clinopyroxene enter the liquid preferentially to olivine and orthopyroxene. Note that the $1 / \mathrm{D}$ limit applies equally to this equation as $F$ becomes small relative to $D_{0}$, since $D_{o}$ and $p$ are usually similar in magnitude.

Chemical variations produced during fractional (in contrast to equilibrium) crystallization and melting, where solid and
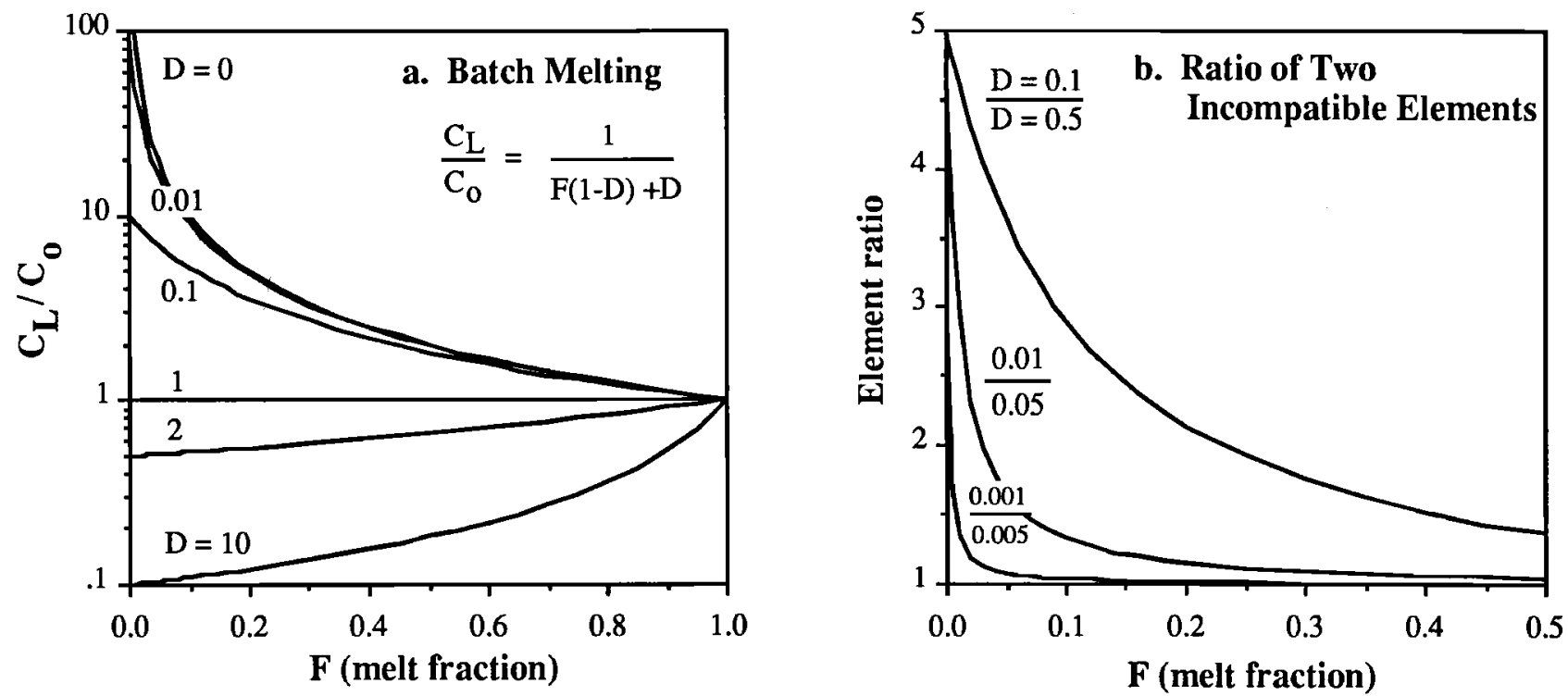

Fig. 4. Illustration of the effects of equilibrium melting on trace element abundances using equation (5) (batch melting equation). a) Variation in liquid concentration $\left(C_{1}\right)$ (normalized to unit source concentration $C_{0}=1$ ) as a function of melt fraction (F) for six "elements" with different bulk distribution coefficients (D). b) Change in the ratios of incompatible elements with different D's as a function of F. Each curve is for a different pair of "elements" that have the D's indicated. Note that for $\mathrm{D}<0.1$, incompatible element ratios can be changed only at very low extents of melting. 


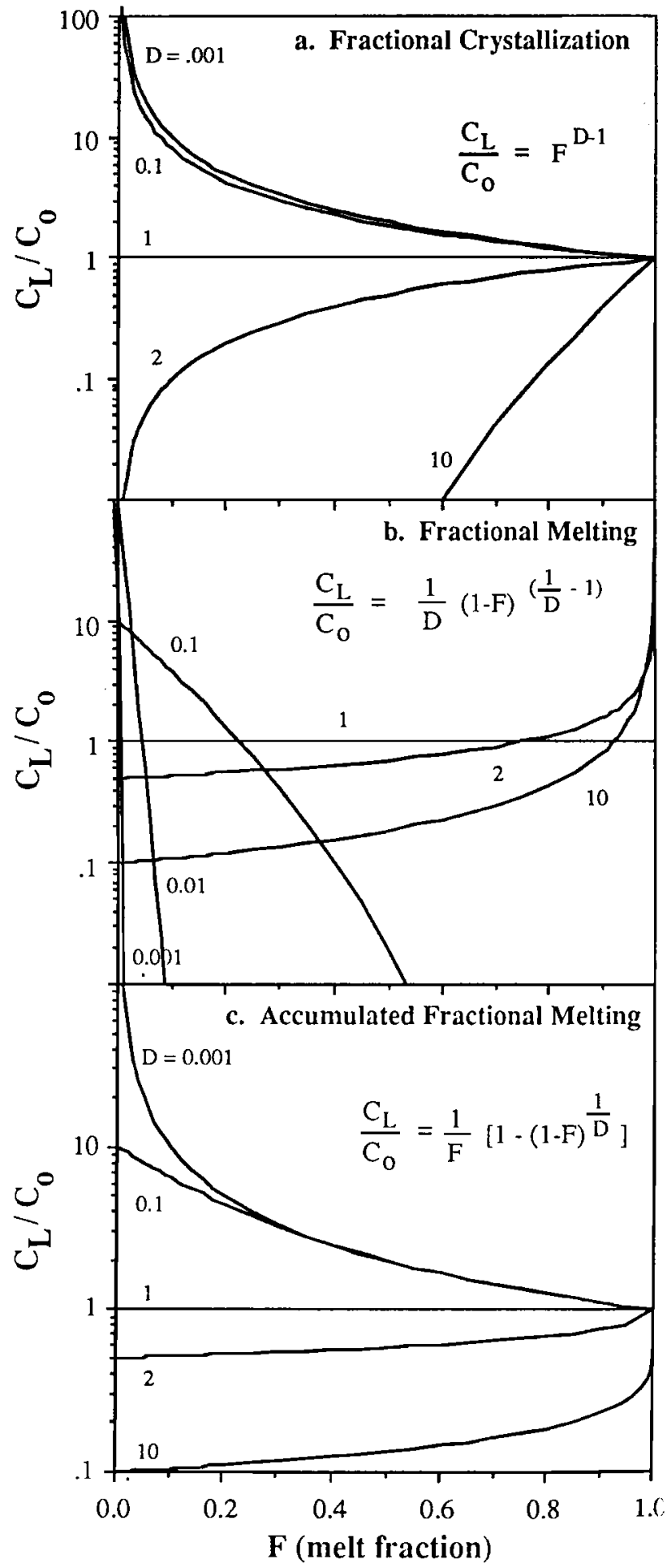

Fig. 5. Illustration of the effects of fractional crystallization and melting on trace element abundances. These results should be compared to Figure 4 - the notation is the same for the two figures. a) Fractional crystallization for five different values of D (equation 7). b) Fractional melting (equation 8). c) Accumulated fractional melting (equation 23). Note the very similar results produced by accumulated fractional melting to the equilibrium melting results of Figure 4. liquid are separated from each other instantaneously throughout the process, are illustrated in Figures $5 a-5 c$. The equations describing fractional crystallization and melting, assuming D remains constant during each process, are respectively [Shaw, 1970]:

$$
\mathrm{C}_{\mathrm{L}} / \mathrm{C}_{\mathrm{o}}=\mathrm{F}^{(\mathrm{D}-1)}
$$

and

$$
C_{L} / C_{o}=(1 / D)^{*}(1-F)^{(1 / D)-1)}
$$

Like equilibrium crystallization, fractional crystallization has little effect on incompatible element ratios, but it causes compatible elements to change even more rapidly with decreasing F. Successive melts produced by fractional melting show strong depletions in incompatible trace elements, due to the rapid removal of these incompatible elements during the first increments of melting. On the other hand, if the melts produced during fractional melting are mixed prior to sampling, the pooled product of fractional melting is nearly identical to a melt produced by equilibrium melting to the same maximum $F$ (Figure 5c).

\subsubsection{Trace Element Patterns}

The chemical systematics presented above lead to straightforward conclusions concerning the significance of trace element variations in MORB. For example, Figure 6 shows three REE patterns for MORB, one typical of "normal" MORB, one from near a hot spot that is highly "enriched", and one that is transitional. Since the LREE are incompatible, the

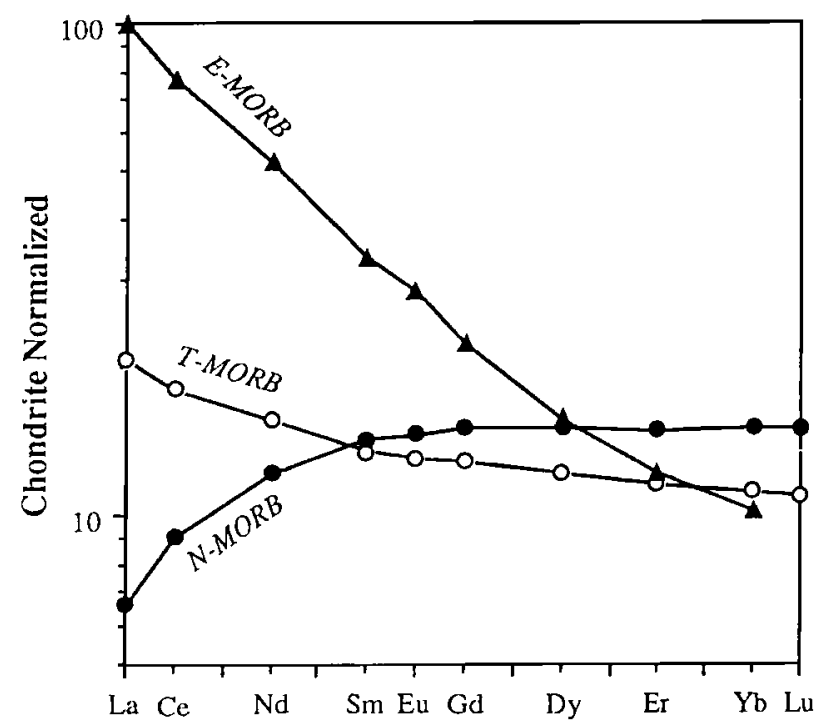

Rare Earth Elements

Fig. 6. Rare earth element patterns for three mi.1-ocean ridge basalts (MORB) from the Mid-Atlantic Ridge (MAR). Inriched E-MORB is from the Azores platform [C.H. Langmuir, unpublished data]; from the Azores platform [C.H. Langmuir, unpublished data]; 1977]; Normal N-MORB is from the MAR, between the Atlantis and Hayes Fracture Zones (MAR standard [LDGO, unpt blished data]). 
mantle source is depleted relative to the melts derived from it (in most cases - see Langmuir et al., 1977). Therefore the LREE depletion of normal MORB requires a LREE depletion of its source. The elevated LREE of the "enriched" MORB requires a source that is enriched relative to that of normal MORB. A conventional trace element interpretation might also be that the enriched MORB is derived by lower extents of melting (leading to higher levels of the relatively incompatible lighter REE). The heavy REE depletion of the enriched MORB suggests that garnet, which preferentially retains the HREE, was a residual phase.

The smooth variations of the REE provide a context for explaining the variations of other trace elements. Two elements that have virtually identical D's maintain a constant ratio through all extents of melting, and may not be fractionated from one another during many of the processes that lead to mantle heterogeneity. For example, $\mathrm{P}$ and $\mathrm{Nd}, \mathrm{Zr}$ and $\mathrm{Sm}, \mathrm{Y}$ and the HREE, and Ti and Eu are all pairs of elements that show a relatively constant ratio in all ocean ridge basalts (Figures $7 a$ and $7 b$, Table 2), which suggests that the D's for each pair of elements are very similar. $\mathrm{P}, \mathrm{Z}_{\mathrm{r}}, \mathrm{Ti}$ and $\mathrm{Y}$ are thus "analogues" for certain of the REE.

In principal, all of the trace elements could be plotted on a single diagram similar to a REE patterns, with the order determined by relative differences in their partition coefficients. These diagrams, commonly called "spidergrams," are often used to present an abundance of trace element data [e.g., Wood, 1979; Sun, 1980; Thompson et al., 1983]. A spidergram is simply a REE diagram such as Figure 6, with other elements added to it. All elements more incompatible than La would be plotted to the left of La. These elements include $\mathrm{Cs}, \mathrm{Pb}, \mathrm{Ba}, \mathrm{Nb}, \mathrm{Ta}, \mathrm{Th}, \mathrm{U}, \mathrm{Rb}$ and $\mathrm{K}$. Analogue elements that behave similarly to the REE would plot in the middle of the REE pattern. Elements more compatible than $\mathrm{Yb}$, such as Sc and V, would plot to the right of the heaviest REE. Spidergrams have the advantage of extending the range of D's that can be examined graphically in a single diagram, and of constructing a REE-like diagram in the absence of REE data. In practice, however, such diagrams often show irregular systematics from element to element, particularly for the most incompatible elements. Nonetheless, the principle behind these diagrams is based on the trace element equations: that a family of elements exhibit systematic relationships that depend primarily on their relative D's, provided the residual mineralogy is similar.

Trace elements provide a widely used method of classification for ocean ridge basalts. Schilling in particular has shown that basalts from near hot spots are relatively enriched in incompatible elements, and that this enrichment often decreased progressively with distance from the hot spot center. This variability in trace element enrichment has led to a simple three-fold descriptive classification for MORB. NMORB (normal) have the characteristic depletion in the most incompatible elements, E-MORB (enriched) are enriched in incompatible elements, and are most prevalent on ridges influenced by hot spots, and T-MORB (transitional) are in between the two. On spidergrams, N-MORB have a positive
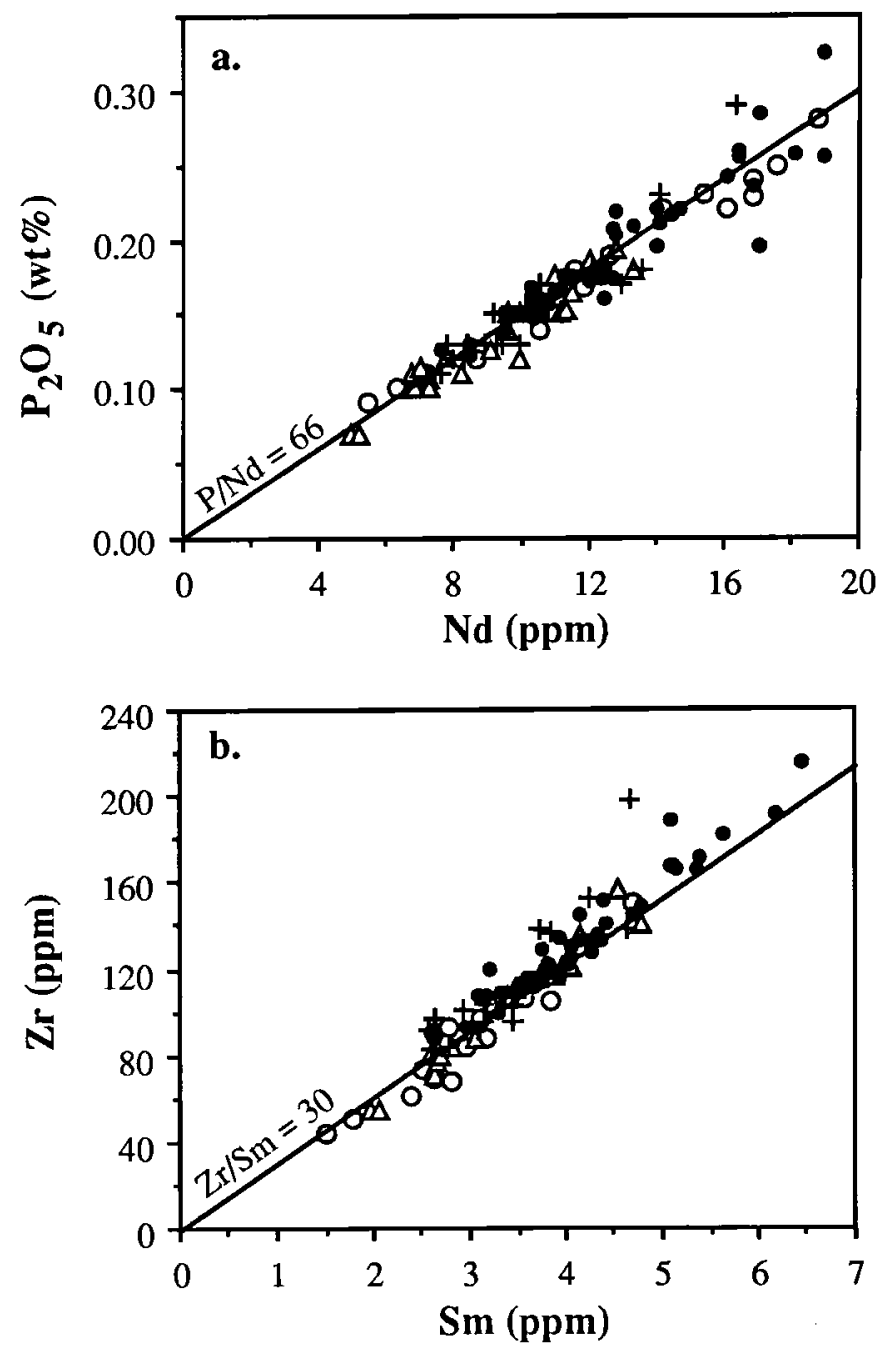

Fig. 7. Analogue element plots showing how some elements behave very similarly to certain REE during the melting and crystallization processes that occur on ocean ridges. a) $\mathrm{P}_{2} \mathrm{O}_{5}$ and $\mathrm{Nd}$, b) $\mathrm{Zr}$ and $\mathrm{Sm}$ concentrations in MORBs. $\mathrm{P}$ and $\mathrm{Zr}$ are analogue elements to the rare earth elements $\mathrm{Nd}$ and $\mathrm{Sm}$, respectively. Plusses for Australian Antarctic Discordance; open circles for Oceanographer Fracture Zone; triangles for Tamayo Fracture Zone; solid circles for CHEPR EPR data. Data sources in Table 2.

slope, T-MORB have a flat slope, and E-MORB have a negative slope.

\section{$2.4 K_{d}$ s and Major Element Equilibria}

Application of the simple batch melting equation to MORB data appears to be problematic because $\mathrm{D}$ can be a function of $\mathrm{T}$, $P$ and $C_{L}$. But at any point in a process, when $T, P$, and $C_{L}$ are fixed, the equation applies. So if we are able to develop equations for $D$ as a function of these variables, we can calculate compositional variations as a function of extent of melting (or crystallization).

Langmuir and Hanson [1980, 1981] showed how the combination of equations from $K_{d}$ 's, mass balance and mineral 
TABLE 2. Analogue Element Ratios for MORB Suites

\begin{tabular}{|c|c|c|c|c|c|c|c|c|c|c|}
\hline & \multirow[b]{2}{*}{$\mathrm{N}$} & \multirow[b]{2}{*}{ Ref. } & \multicolumn{2}{|c|}{$\mathrm{P} / \mathrm{Nd}$} & \multicolumn{2}{|c|}{$\mathrm{Zr} / \mathrm{Sm}$} & \multicolumn{2}{|c|}{$\mathrm{Ti} / \mathrm{Eu}^{*}$} & \multicolumn{2}{|c|}{$\mathrm{Y} / \mathrm{Yb}$} \\
\hline & & & Mean & $\mathrm{SD}$ & Mean & $S D$ & Mean & $\mathrm{SD}$ & Mean & $\mathrm{SD}$ \\
\hline Oceanographer & 19 & 1,2 & 65 & 5 & 29 & 2.7 & 6876 & 261 & 10.7 & 0.6 \\
\hline Tamayo & 29 & 2,3 & 63 & 4 & 29.9 & 2 & 6939 & 224 & 10.2 & 0.4 \\
\hline CHEPR & 48 & $4,2,5$ & 65 & 5 & 32.1 & 1.7 & 6853 & 279 & 10.2 & 0.4 \\
\hline FAMOUS & 12 & 4,2 & 69 & 3 & 30.5 & 3.9 & 6875 & 279 & 10.4 & 0.6 \\
\hline $\mathrm{AAD}$ & 20 & 6 & 65 & 6 & 33 & 3.8 & 6695 & 493 & 10.2 & 0.7 \\
\hline Kolbeinsey & 23 & 7 & 62 & 9 & -- & --- & 6662 & 447 & -- & -- \\
\hline Galapagos & 10 & 8 & 59 & 22 & 28.9 & 2.8 & 6820 & 916 & 10.8 & 0.8 \\
\hline TOTAL & 161 & & 64.0 & 3.2 & 30.6 & 1.7 & 6817 & 101.61 & 10.4 & .27 \\
\hline ID/DCP only & 108 & & 65.7 & 2.4 & 30.4 & 1.3 & 6886 & 26 & 10.4 & 0.24 \\
\hline
\end{tabular}

$\begin{array}{lll}\text { References: } & \begin{array}{l}\text { 1 Shirey et al., } 1987 \\ \text { 2 Bender and Langmuir, } \\ \text { unpublished data }\end{array} & 6 \text { Langmuir et al., } 1986 \\ & \text { 3 Bender et al., 1984 et al., 1991 } \\ \text { 4 Langmuir et al., 1977 } & 7 \text { Schilling et al., 1981 } \\ & 8 \text { Perfit et al., 1983 }\end{array}$

stoichiometry can be used for the general calculation of multiphase equilibria. They showed that, given $K_{d}$ 's and a bulk composition, the stability, proportions and compositions of all phases in the system can be uniquely determined. Obviously, this approach allows calculation of liquid compositions during either crystallization or melting, if expressions for the $K_{d}$ 's are known. We present here a simple illustration of the approach, using olivine, because the constraints from olivine are important for understanding the chemical consequences of mantle melting.

The olivine formula $(\mathrm{Mg}, \mathrm{Fe})_{2} \mathrm{SiO}_{4}$ imposes a stoichiometric constraint in terms of cation fractions:

$$
\mathrm{C}_{\text {olivine }}^{\text {MgO }}+\mathrm{C}_{\text {olivine }}^{\mathrm{FeO}}=0.6667
$$

Roeder and Emslie [1970] showed that for a limited compositional range the partition coefficients between olivine and liquid have a simple linear dependence on temperature:

$$
\begin{aligned}
& \log \mathrm{K}_{\mathrm{dMgO}}=3740 / \mathrm{T}-1.87 \\
& \log \mathrm{K}_{\mathrm{dFeO}}=3911 / \mathrm{T}-2.50
\end{aligned}
$$

(We will see below that the compositional dependencies become important for mantle melting. Variations of olivine $\mathrm{K}_{\mathrm{d}}$ 's with temperature, pressure, and liquid composition are presented in Appendix B). Since the temperature dependence of the two $K_{d}$ 's is very similar, the ratio of the two is nearly constant. This ratio, $K_{D}$, is about 0.3 , and is relatively insensitive to changes in $T, P$ or composition in the geological conditions of interest. $K_{D}$ is defined as

$$
\mathrm{K}_{\mathrm{D}}=\left[\mathrm{C}_{\mathrm{al}}^{\mathrm{FoO}} / \mathrm{C}_{\mathrm{L}}^{\mathrm{FeO}}\right] /\left[\mathrm{C}_{\mathrm{ol}}^{\mathrm{MgO}} / \mathrm{C}_{\mathrm{L}}^{\mathrm{MgO}}\right]
$$

From the definition of $K_{d}$ in equation (1):

$$
\begin{aligned}
& \mathrm{K}_{\mathrm{dMgO}}^{\mathrm{O} / \mathrm{L}}=\mathrm{C}_{\mathrm{s}}^{\mathrm{MgO}} / \mathrm{C}_{\mathrm{L}}^{\mathrm{MgO}} \\
& \mathrm{K}_{\mathrm{dF} \mathrm{OO}}^{\mathrm{O}}=\mathrm{C}_{\mathrm{s}}^{\mathrm{FeO}} / \mathrm{C}_{\mathrm{L}}^{\mathrm{FeO}}
\end{aligned}
$$

Substituting these expressions for $\mathrm{C}_{\mathrm{s}}$, and the generalized $\mathrm{K}_{\mathrm{d}}$ expressions of equations (10) and (11) into equation (9) yields the following relation:

$$
\mathrm{C}_{\mathrm{L}}^{\mathrm{MgO}} * 10^{(\mathrm{A} 1 / \mathrm{T})-\mathrm{B} 1}+\mathrm{C}_{\mathrm{L}}^{\mathrm{FeO}} * 10^{(\mathrm{A} 2 / \mathrm{T})-\mathrm{B} 2}=0.6667
$$

where $A 1, A 2, B 1$ and $B 2$ are constants.

This is the equation for the olivine saturation surface first presented by Roeder and Emslie [1970], and provides the temperature at which any given liquid composition will be in equilibrium with olivine, and the composition of the olivine. The equation represents the olivine-liquid phase diagram, for real basaltic systems. A similar equation could also be written that would include complex compositional dependences - the $\mathrm{K}_{\mathrm{d}}$ expressions simply become more complicated. Simultaneous solution for many phases gives phase compositions and proportions for any bulk composition at any $P$ and $T$ for which $K_{d}$ 's are known.

The olivine saturation surface is particularly useful for our purposes because olivine is the dominant mineral in the mantle, and all mantle melts are in equilibrium with olivine. Since the olivine composition in the mantle source is well known, all initial liquids must be in equilibrium with an olivine of this particular composition, and lie on the olivine saturation surface at the $P$ and $T$ of interest. The fact that $K_{D}$ is constant requires (equation (12)) that the $\mathrm{Fe} / \mathrm{Mg}$ ratio of the liquid is fixed. Figure 8 presents a simplified illustration of the olivine 


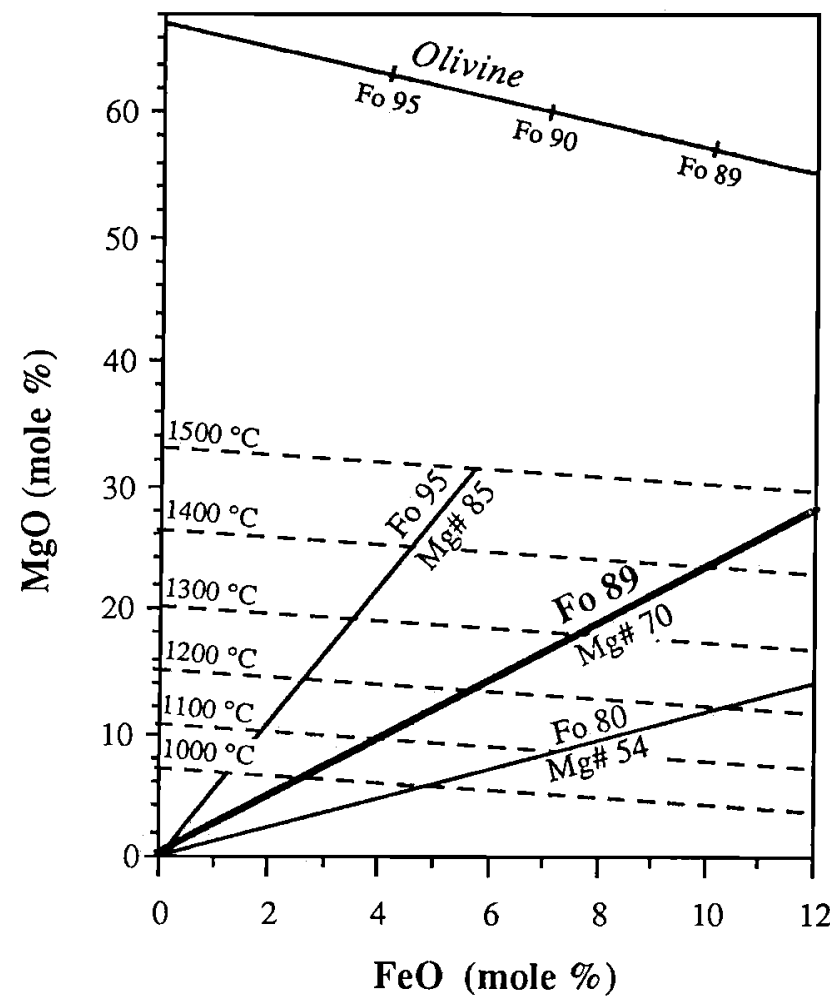

Fig. 8. Olivine saturation surface using the data of Roeder and Emslie [1970] and after Langmuir and Hanson [1981]. The forsterite (Fo) compositions above the lines radiating from the origin are olivine compositions that coexist with liquid compositions along the line $(\mathrm{Mg} \#=\mathrm{MgO} /[\mathrm{MgO}+\mathrm{FeO}]$ in cation mole\%).

saturation surface. Melts in equilibrium with a mantle containing $\mathrm{Fo}_{89}$ olivine must plot on the line labelled $\mathrm{Fo}_{89}$. Higher pressure melts of the mantle plot along the $\mathrm{Fo}_{89}$ melt line toward higher $\mathrm{MgO}$ and $\mathrm{FeO}$ contents. Crystallization of olivine from the melt leads to almost vertical trends on this diagram (until very high degrees of crystallization); therefore, provided olivine is the only mineral that has crystallized en route to the surface, liquids formed at higher pressures remain higher in $\mathrm{FeO}$ than those formed at lower pressures.

These simple principles of element partitioning provide a useful framework in which the observed variations of some elements in oceanic basalts can be understood. The behavior of the elements in more complex and open system models will be considered further in section 4.0. A more sophisticated approach that allows calculation of $\mathrm{FeO}$ and $\mathrm{MgO}$ in mantle melts for a variety of conditions and processes will be discussed at length in section 5.0.

\section{OBSERVATIONS}

\subsection{Influence of Low Pressure Fractionation on MORB Compositions.}

The chemical changes that occur during low-pressure crystallization are well documented by abundant experimental data [e.g., Bender et al., 1978; Walker et al., 1979; Fisk et al., 1980; Grove and Bryan, 1983]. Computer programs are available that use the experimental data to calculate the changes in melt composition produced during crystallization, also called the liquid lines of descent [LLD; Langmuir and Hanson, 1981; Ghiorso et al., 1983; Nielsen, 1985; 1990; Weaver and Langmuir, 1990; Longhi, 1991]. The programs generally are calibrated using a large experimental data base from many laboratories, and problems of interlaboratory biases commonly lead to errors in the temperatures of phase appearances of $10^{\circ}-15^{\circ} \mathrm{C}$. Such errors make the programs useful for general considerations of crystallization, but inadequate for detailed petrogenetic modeling of an individual rock suite. For this latter purpose, the program must be "tuned" to the data set of interest, preferably by having experiments on the same samples, analyzed in the same laboratory as the experiments themselves. Many of the programs have an advantage over experiments, however, in that bulk composition is preserved, and there are no kinetic effects. Thus LLDs calculated on the basis of experimental results may more accurately reflect crystallization processes than the experiments themselves.

Examples of $1 \mathrm{~atm}$ LLDs calculated for a high $\mathrm{MgO}$ sample from the East Pacific Rise near the Clipperton transform fault using the programs of Weaver and Langmuir [1990] and Nielsen [1985] are shown in Figure 9. The LLD's generally track the trends of the data, suggesting that these samples are related, to first order, by low-pressure fractionation.

The fit of both calculated LLDs in Figure 9 to the basalt data is particularly good for elements like $\mathrm{Na}_{2} \mathrm{O}$ and $\mathrm{TiO}_{2}$ that are incompatible with respect to the fractionating phases. Elements whose variations are strongly linked to a single mineral phase (such as $\mathrm{Ca}$ to clinopyroxene or $\mathrm{Al}$ to plagioclase) are more sensitive to the detailed fit of the calculated LLD. Note, for example, that both calculated LLDs in Figure 9 show a marked decrease in $\mathrm{CaO}$ with the arrival of clinopyroxene on the liquidus, producing a kink in each trend. The two LLDs differ in the precise position of the kink on this diagram due to differences in the clinopyroxene $K_{d}$ relationships in the two programs. The offset, however, represents only a $12^{\circ} \mathrm{C}$ difference in the appearance of clinopyroxene on the liquidus, and this temperature difference is within the combined uncertainties in the experimental and analytical measurements that direct the programs. The fact that a mere $12^{\circ}$ difference in the appearance of a phase can significantly affect the systematics of sensitive elements is the reason that detailed modeling of data from a particular region requires a program that is well calibrated by experimental data for those particular samples. This example underscores the importance of "tuning" the LLD program to the data set of interest for detailed petrogenetic modeling.

These diagrams illustrate some of the simplest and yet most important observations concerning MORB. The first is that samples from individual regions usually form rather smooth trends on plots of elemental (or oxide) abundances as a function of $\mathrm{MgO}$. Petrologists often plot elemental variations as a function of $\mathrm{MgO}$ (or $\mathrm{Mg} \#=\mathrm{Mg} /(\mathrm{Mg}+\mathrm{Fe}$ ) in cation mole \%) 

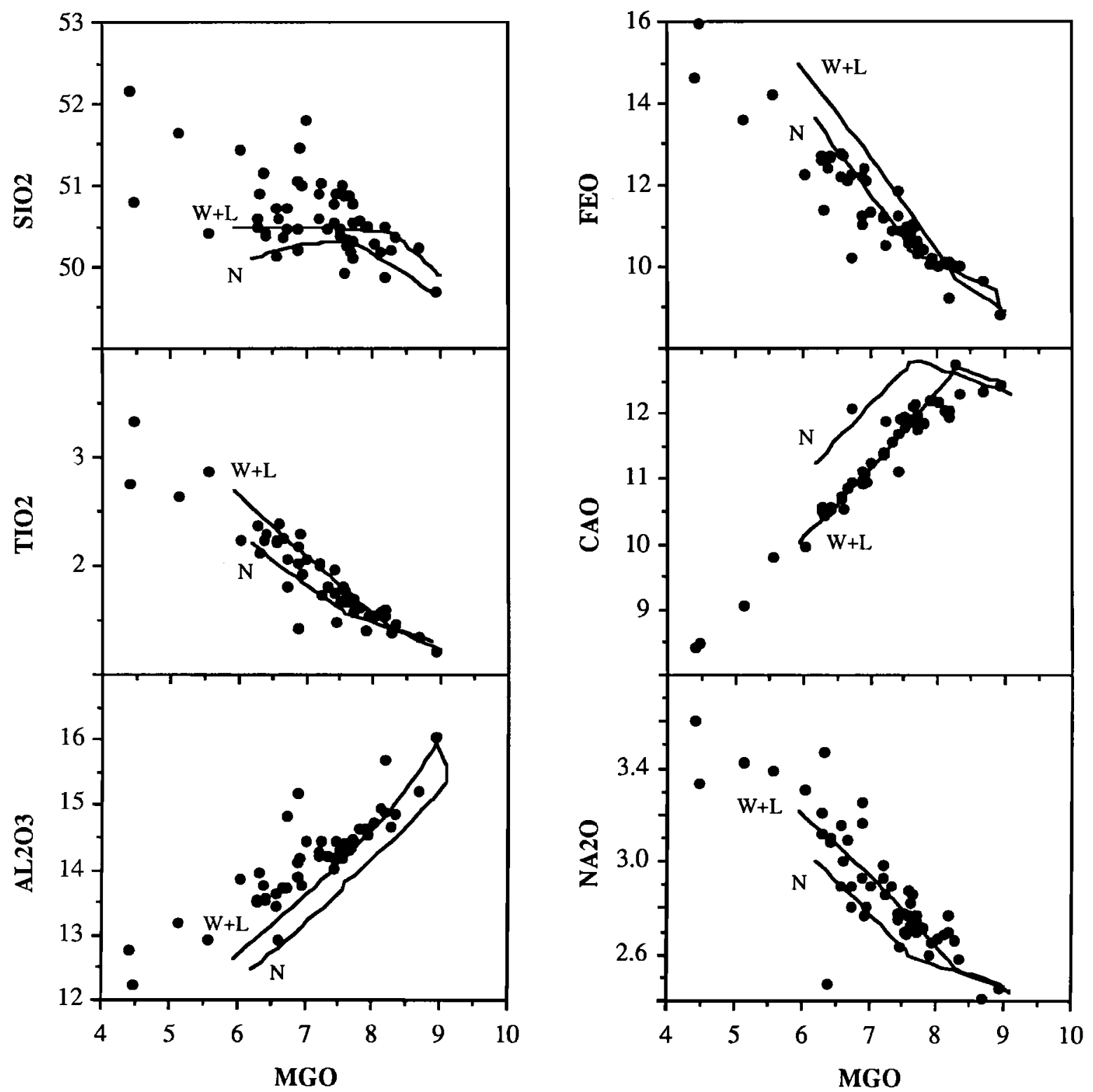

Fig. 9. $\mathrm{MgO}$ variation diagrams for microprobe data for basalt glasses from near the Clipperton Fracture Zone [J. $F$. Bender and C. H. Langmuir, unpublished data]. Lines are calculated liquid lines of descent (LLD) using the programs of Weaver and Langmuir [1990] (labeled W+L) and Nielsen [1985] (labeled N) for the primitive composition at around $9 \mathrm{wt} \%$ $\mathrm{MgO}$. A more recent version of Nielsen's LLD program can be found in Nielsen [1990]. Oxides in wt\%.

because $\mathrm{MgO}$ is compatible in olivine and therefore decreases during cooling and crystallization at low-pressure. Indeed, there is a simple relationship between $\mathrm{MgO}$ contents and magmatic temperature, as shown in Figure 10. Rocks with the highest $\mathrm{MgO}$ are the highest temperature magmas and so represent compositions least modified from primary mantle melts. However, as Figures 9 and 10 make clear, the vast majority of recovered basalts have experienced substantial cooling since their generation within the mantle, and this cooling has produced changes in the concentrations of all the elements. These chemical changes must be corrected for if we are to see through the substantial chemical variability caused by differentiation processes at low pressure.

Examination of data from many regions of the ocean floor shows the ubiquitous influence of the low-pressure fractionation signature on MORB composicions. Figures $11 a$ and $11 b$ shows $\mathrm{MgO}$ and $\mathrm{Na}_{2} \mathrm{O}$ variations in samples from five regions that encompass most of the global range in $\mathrm{Na}_{2} \mathrm{O}$ contents for MORBs. The data from the five regions form subparallel trends, and within each region, the $\mathrm{Na}_{2} \mathrm{O}$ contents 


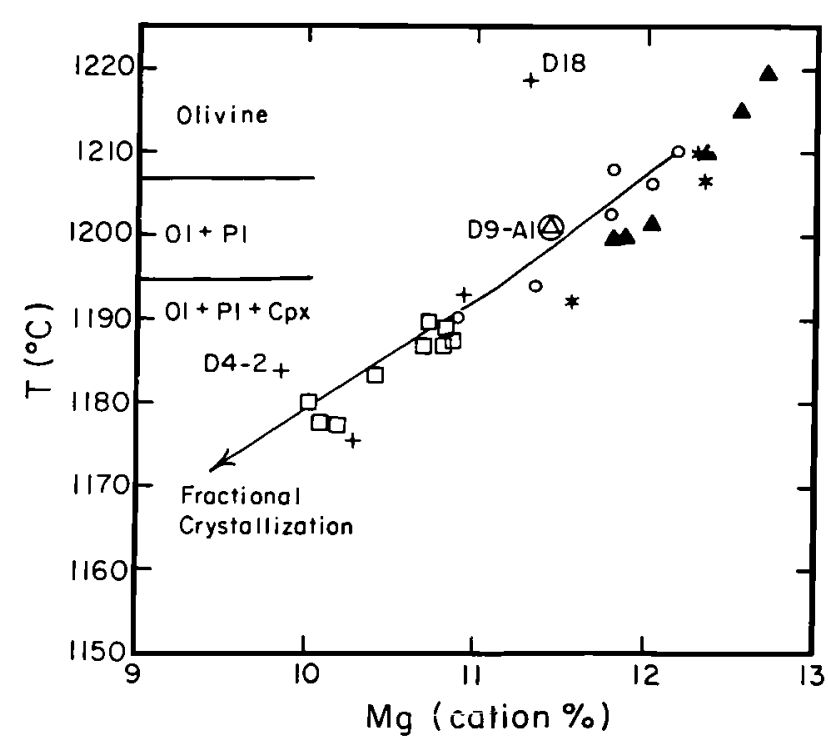

Fig. 10. Calculated liquidus temperatures for basalt glasses from the Tamayo region of the East Pacific Rise vs. MgO. The LLD for one of the samples is shown for reference. LLD, liquidus temperatures, and phase appearances are calculated from the method of Weaver and Langmuir [1990]. Figure is from Bender et al. [1984].

increase gradually with decreasing $\mathrm{MgO}$ contents. The trends of the data for each region are similar to calculated low-pressure LLDs.

In addition to the changes in melt ccmposition produced during low-pressure crystallization, it is clear from the data presented in Figures $11 a$ and $11 b$ that there are at least two other aspects of chemical variability. First, the five regions form sub-parallel trends, so that at a given $\mathrm{MgO}$ content, each region shows a distinct and characteristic narrow range in $\mathrm{Na}_{2} \mathrm{O}$ contents. Anticipating a topic that will be discussed in greater detail below, these differences in $\mathrm{Na}_{2} \mathrm{O}$ contents (and other elements) at a given value of $\mathrm{MgO}$ from region to region along the mid-ocean ridge system appear to reflect global variations in parental magma composition. Second, it is apparent that even in the data from a single region, there is variation in $\mathrm{Na}_{2} \mathrm{O}$ (and other elements) at a single value of $\mathrm{MgO}$. While some component of this local variability may result from analytica! error, there are often compositional systematics to this variability as well (shown below).

To summarize, we can divide the observed compositional variations into three categories. First, there is the variability that appears to result largely from low-pressure crystallization and varies systematically with $\mathrm{MgO}$. Second, among samples collected from an individual ridge segment, there is local chemical variability that cannot be accounted for by lowpressure crystallization. Last, there is global chemical variability manifest as differences in average compositions (at a given $\mathrm{MgO}$ content) from region to region. Figure 12 illustrates the three levels of compositional variability: the lines show low pressure fractionation, the shaded boxes show the local variability at a given value of $\mathrm{MgO}$, and the two open circles show the average difference between the two regions at a given value of $\mathrm{MgO}$. Because the chemical changes produced by low-pressure crystallization obscure the local and global chemical systematics, it is necessary to correct the basalt data for the effects of differentiation. In essence, we want to determine the residual signal left after low-pressure differentiation. It is this residual signa. that must then be accounted for either by some more complex fractionation process, by processes of melt generation and segregation, or by source heterogeneity.

\subsection{Correction of Data for Low Pressure Fractionation}

The existence of sub-parallel trends for the $\mathrm{Na}_{2} \mathrm{O}$ LLDs (e.g., Figures $11 a$ and $11 b$ ) provides a ready basis for "correcting" the data for the effects of low pressure fractionation. Figure $11 a$ illustrates the method used by $K L 87$ where $\mathrm{Na}_{2} \mathrm{O}$ values are extrapolated along a constant slope to $8 \mathrm{wt} \% \mathrm{MgO}$ (the calculated value of $\mathrm{Na}_{2} \mathrm{O}$ at $8 \mathrm{wt} \% \mathrm{MgO}$ is called $\left.\mathrm{Na}_{\mathbf{8} .0}\right)$. This constant slope is meant to approximate the slope of the olivine+plagioclase \pm clinopyroxene LLD. In this way, samples that lie along a perfectly linear fractionation trend of this slope would collapse to a single value of $\mathrm{Na}_{8.0}$. Figure $11 a$ shows the slope of the line used by $K L 87$ to calculate $\mathrm{Na}_{8.0}$, superimposed on five MORB suites. The constant slope method is clearly not appropriate for all data sets. For some it approximates the trend of the data and so a calculated value of $\mathrm{Na}_{8.0}$ is similar to the real $\mathrm{Na}_{2} \mathrm{O}$ contents of samples with $8 \mathrm{wt} \%$ $\mathrm{MgO}$. For other regions, however, there are substantial differences between the slopes of the real data, and the slope used in the algorithm, so that calculated values of $\mathrm{Na}_{8.0}$ give inaccurate estimates of $\mathrm{Na}_{2} \mathrm{O}$ at $8 \mathrm{wt} \% \mathrm{MgO}$.

One problem with the constant slope used to normalize the data is that the slope of a fractionation trend varies naturally as a function of the composition of each MORB suite and is steeper with increasing $\mathrm{Na}_{2} \mathrm{O}$ contents from region to region (Figure $11 b$ ). KL87 recognized this problem for the low $\mathrm{Na}_{2} \mathrm{O}$ samples from the Kolbeinsey Ridge, which fall on a shallower trend than that assumed in the $\mathrm{Na}_{8.0}$ calculation, but did not take this differences into account. Thus the $\mathrm{Na}_{8.0}$ values calculated for fractionated samples from the Kolbeinsey Ridge are lower than the real $\mathrm{Na}_{2} \mathrm{O}$ contents of samples with $8 \mathrm{wt} \% \mathrm{MgO}$.

Another problem with the constant slope used for fractionation correction is that an abrupt change in slope often occurs when a new mineral joins the fractionating assemblage. For example, there is a slight kink in $\mathrm{Na}_{2} \mathrm{O}-\mathrm{MgO}$ LLDs where clinopyroxene joins olivine and plagioclase as a fractionating phase (Figure 11b). This change in slope is apparent in some of the natural suites as well; samples above $8.0 \mathrm{wt} \% \mathrm{MgO}$ from the ridges adjacent to the Kane Fracture Zone, for example, lie on a shallow slope reflecting only olivine and plagioclase crystallization (Figures $11 a$ and $11 b$ ). Because the $\mathrm{Na}_{8.0}$ equation from $K L 87$ was based on a steeper olivineplagioclase-clinopyroxene fractionation trend, the $\mathrm{Na}_{8.0}$ values calculated from this equation will be erroneously high for the high $\mathrm{MgO}$ Kane samples.

Thus, the variations in slope predicted by both changes in starting composition and position along the LLD could lead to 


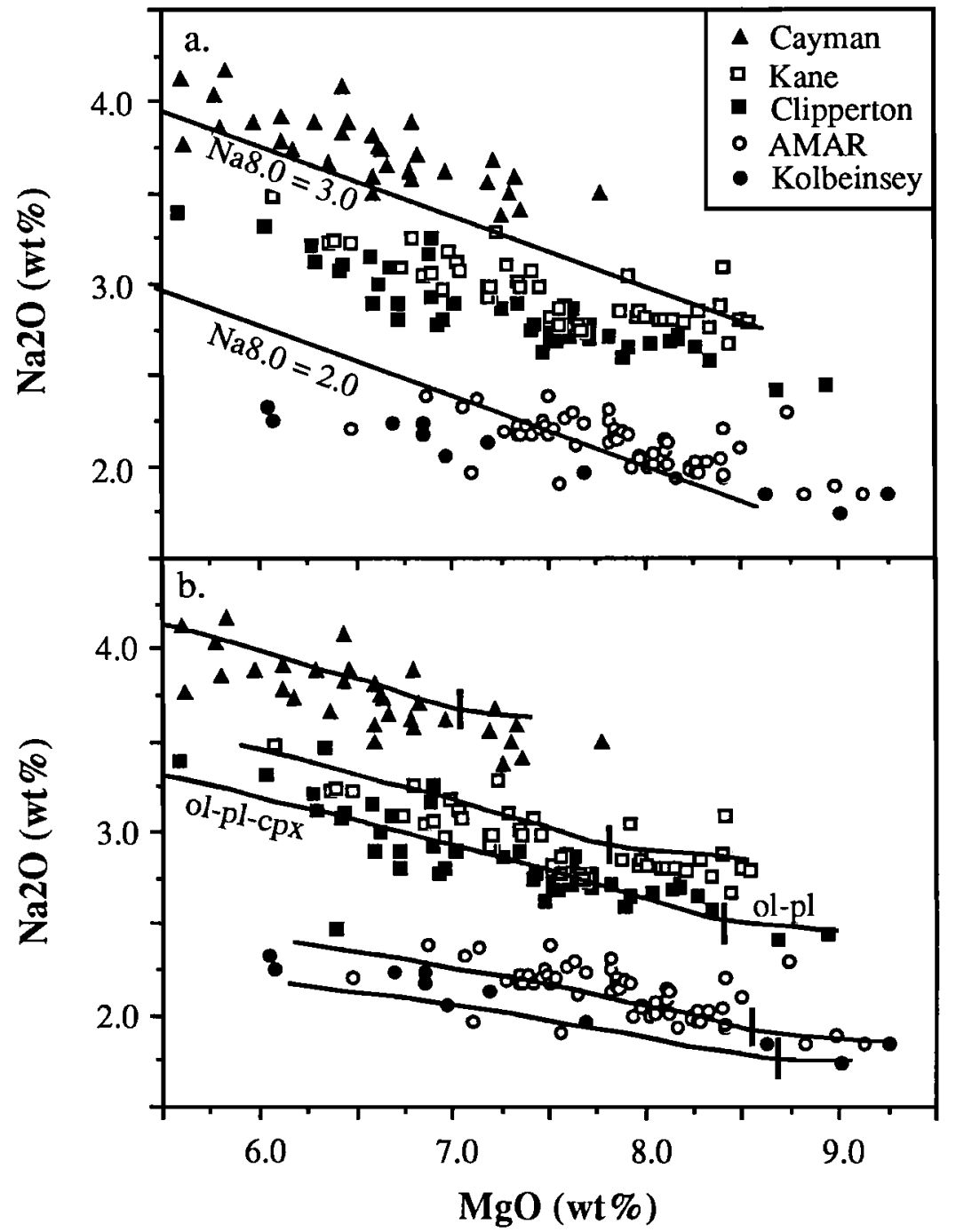

Fig. 11. a) $\mathrm{MgO}$ vs. $\mathrm{Na}_{2} \mathrm{O}$ in MORBs from five regions. Samples are from the Mid-Cayman Rise, from near the Kane Fracture Zone at $23.22-23.67^{\circ} \mathrm{N}$ on the Mid-Atlantic Ridge (MAR), from the AMAR region at $36.42-36.79^{\circ} \mathrm{N}$ on the MAR, from near the Clipperton Fracture Zone on the EPR, and from the Kolbeinsey Ridge north of Iceland. Data from the Smithsonian Catalogue of Basalt Glasses [Melson et al., 1977; and pers. comm.], except for Clipperton data (as in Figure 9), and Kolbeinsey data [Schilling et al., 1983]. Lines show slope used by Klein and Langmuir [1987] to calculate $\mathrm{Na}_{8.0}$ $\left(\mathrm{Na}_{2} \mathrm{O}\right.$ at $8 \% \mathrm{MgO}$ ) b) bame data with calculated LLDs for a primitive composition from each of the five suites using the program of Weaver and Langmuir [1990]. Vertical bar indicates the point at which clinopyroxene (cpx) joins olivine and plagioclase (ol-pl) as a fractionating phase, at which point the slope of the LLD steepens.

emors in $\mathrm{Na}_{8.0}$ values calculated assuming a constant slope. It is difficult, however, to develop a single algorithm that takes into account these effects. One way to improve upon the constant slope method would be to use the LLD program to develop a $\mathrm{Na}_{8.0}$ algorithm specific to the region of interest. For example, in modeling samples from the Kane region, different slopes could be adopted on either side of the clinopyroxene-in kink at $8 \% \mathrm{MgO}$. Samples with greater Lian $8 \% \mathrm{MgO}$ can be projected to $8 \% \mathrm{MgO}$ along the shallow olivine-plagioclase slope, and the samples with less than $8 \% \mathrm{MgO}$ along the steeper olivine-plagioclase-clinopyroxe te slope. Because local variability is defined as the deviation from a low pressure LLD, it is imperative that one pay particular attention to the details of the LLD in modeling local variations [Klein and Langmuir, 1989].

Will a more accurate $\mathrm{Na}_{8.0}$ correction affect the global $\mathrm{Na}_{8.0}$ variations as well? In order to assess any artifacts in the $\mathrm{Na}_{8.0}$ global averaging that may have resulted from the constant slope method used by $K L 87$, we have recalculated $\mathrm{Na}_{8.0}$ averages for the five suites shown in Figures $11 a$ and $11 b$ using the slopes and kinks given in the LLDs calculated for those regions. These results are shown in Figure 13, along with the 


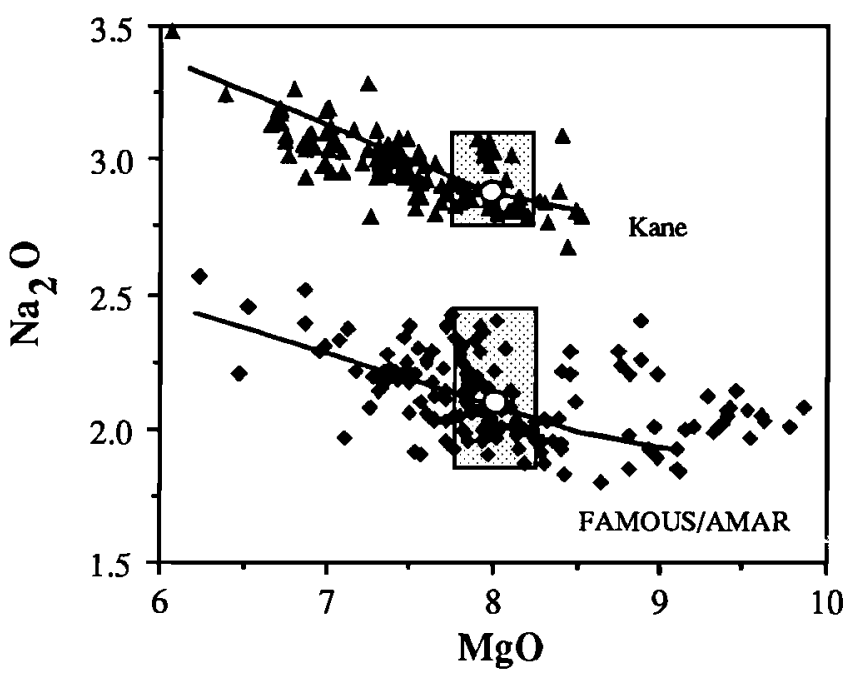

Fig. 12. $\mathrm{MgO}$ vs. $\mathrm{Na}_{2} \mathrm{O}$ for $\mathrm{Kane}$ and FAMOUS/AMAR basalt glasses. Data from Smithsonian Catalogue of Basalt Glasses [Melson et al., 1977; and pers. comm.]. Lines are LLD's for one composition from each of the suites. Shaded bar is variability in each of the suites at $\sim 8$ $\mathrm{wt} \% \mathrm{MgO}$. Open circle is the average $\mathrm{Na}_{2} \mathrm{O}$ content at $8 \mathrm{wt} \% \mathrm{MgO}$ for each suite. Thus there are three levels of variability in the data: variability due to low-pressure crystal fractionation, local variability at a constant $\mathrm{MgO}$ value, and average differences among different regions that contribute to a global signal.

original determinations of $\mathrm{Na}_{8.0}$ using the $K L 87$ method. All of the new $\mathrm{Na}_{8.0}$ averages are higher than the original ones. This is because the constant slope (0.373) used is often too steep for the data, and so overcorrects the $\mathrm{Na}_{2} \mathrm{O}$ values of fractionated samples. For example, the constant slope of 0.373 is too steep for both the entire Kolbeinsey suite, and the region of the Cayman LLD above $7 \% \mathrm{MgO}$ where only olivine and plagioclase fractionate. Taking into account these complexities for the Kolbeinsey and Cayman LLDs, leads to higher average $\mathrm{Na}_{8.0}$ values for both regions.

The change in the $\mathrm{Na}_{8.0}$ averages using the new and more accurate correction scheme, however, is typically only $0.2 \mathrm{wt} \%$ $\mathrm{Na}_{2} \mathrm{O}$, and most importantly, the relative changes among the regions are essentially the same. Because these effects are small and systematic, we have continued to use the $K L 87$ correction scheme for global averages in this paper. The local systematics, however, have been investigated using the LLD appropriate for each particular region.

The method presented above for correcting $\mathrm{Na}_{2} \mathrm{O}$ values for fractionation can be developed for any element that varies predictably with differentiation such as $\mathrm{FeO}$ and $\mathrm{SiO}_{2}$ (see $K L 87$ and Klein and Langmuir, 1989, for the algorithms used to calculate $\mathrm{Fe}_{8.0}$ and $\mathrm{Si}_{8,0}$ ). Since incomfatible trace elements vary inversely with $F$, and are insensitive to the appearances of specific mineral phases, an equation can be derived simply from the relationship between $\mathrm{F}$ and $\mathrm{MgO}$ given by the LLD program to correct highly incompatible elements for fractionation. Such an equation for Ce, for example, is:

$$
\log \left(\mathrm{Ce}_{8.0}\right)=\log (\mathrm{Ce})-0.11 *(8 \cdot \mathrm{MgO})
$$

\subsubsection{Interlaboratory Bias}

Different laboratories calibrate their analyses to different standards, which often leads to a significant and systematic offset between analyses from different laboratories. Because these interlaboratory differences can be large, it is important to apply corrections to the various data in order to make them consistent with one another. The largest number of analyses of MORB have been determined by electron microprobe at the Smithsonian Institution in the laboratory of William Melson. Two other important sources of data for this paper are the analyses from Jean-Guy Schilling at the University of Rhode Island, and more recent analyses at Lamont.

Two independent approaches provide information on the necessary interlaboratory correction factors. There has been sufficient overlap in the samples analyzed by electron microprobe at the Smithsonian, and by microprobe and direct current plasma spectrometry at Lamont to determine systematic biases between these laboratories [e.g., Klein et al., 1991]. This leads to correction factors for each element that can be applied to individual analyses. These factors cause $\mathrm{Na}_{8.0}$ and $\mathrm{Fe}_{8.0}$ calculated from Lamont data to be respectively $6-8 \%$ and 3 $7 \%$ higher than the same samples measured at the Smithsonian.

It is also possible to compare regional averages for groups of samples determined by the different methods. This latter approach can also be used to evaluate the Rhode Island data. Figure 14 compares regionally averaged data from seven different ridge segments. Both the Lamont and Rhode Island data are similarly offset from the Smithsonian data. The offsets in the regional averages are similar to those calculated from the elemental correction factors for individual analyses (as above). In the following presentation of MORB data, we have applied

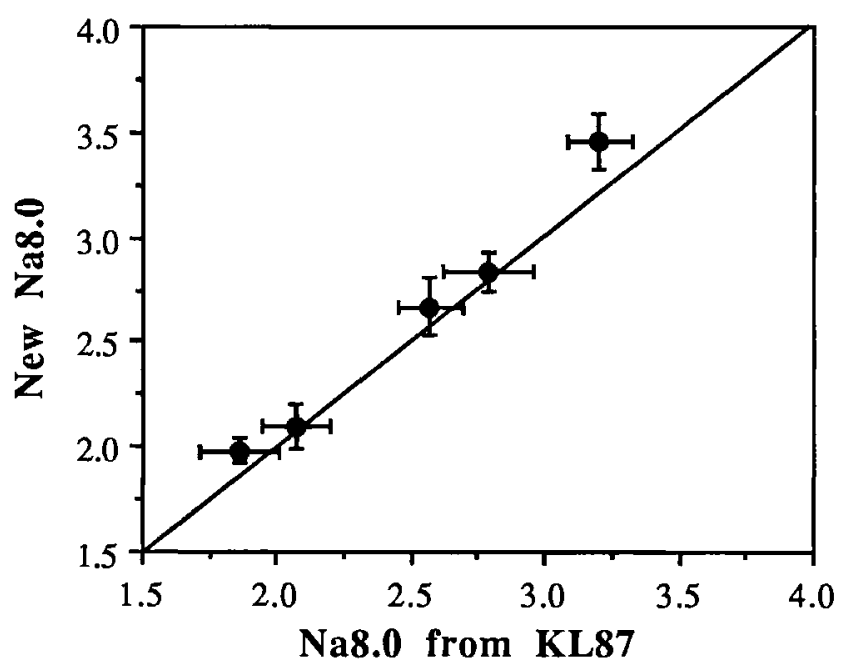

Fig. 13. $\mathrm{Na}_{\mathbf{8 . 0}}$ for the five suites shown in Figure 11 . The original values given in Klein and Langmuir [1987] are compared to those calculated by taking into account the slopes of the individual LLDs shown in Figure $11 b$. Each point is an average of the $\mathrm{Na}_{8,0}$ values for each region, and the error bars are one standard deviation of the $\mathrm{Na}_{8.0}$ values. 


\section{REGIONAL AVERAGES}
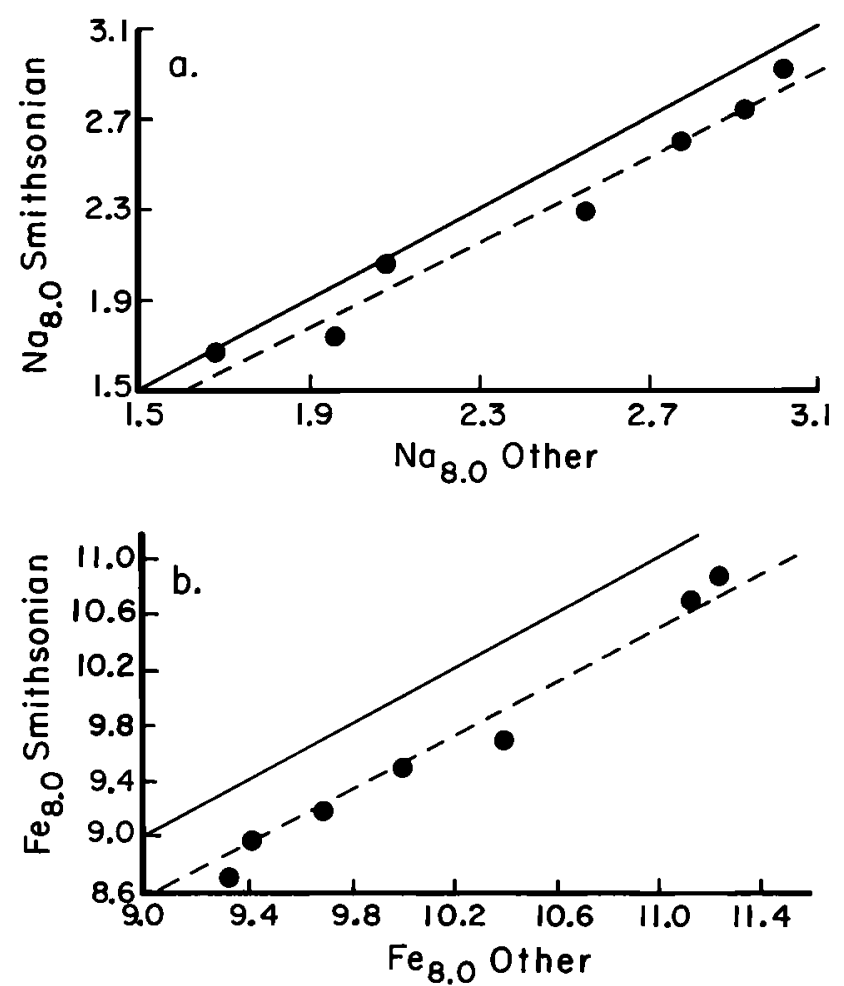

Fig. 14. A comparison of regional mean $a$ ) $\mathrm{Na}_{8.0}$ and $b$ ) $\mathrm{Fe}_{8.0}$ calculated from measurements by different laboratories. Ths individual samples are not identical in all cases, so the comparisen includes sampling biases as well as calibration differences among the laboratories and analytical methods. "Smithsonian" data is by nicroprobe analysis [e.g., Melson et al., 1977]. "Other" data is by nicroprobe or direct current plasma emission spectrometry at Lamont [e.g., Klein et al., 1991] or by classical chemical methods at J.-G. Schilling's laboratory at the University of Rhode Island [e.g., Schilling et al., 1983]. The data points, which span most of the global range, are from the following regions: Reykjanes Ridge (2), EPR near the Tamayo Transform, S. Kane, FAMOUS, and the Australian Antarctic Discordance (2). Solid line is the line for perfect agreement; dashed line through the data is a line for a constant percentage change. The interlaboratory correction factor is $7.5 \%$ for $\mathrm{Na}_{8.0}$ and $5 \%$ for $\mathrm{Fe}_{8.0}$.

corrections to analyses such that they are consistent with Lamont and Rhode Island analyses. Applying these corrections reveals systematics in the data that are not apparent in the uncorrected data.

\subsection{Global Variability of Major Elements in MORB}

Our examination of the major element systematics observed among mid-ocean ridge basalts begins with the systematics observed on a global scale, for regionally averaged data. For more than a decade, petrologists have noted systematic differences in MORB major element chemistry from region to region. Early, predominantly reconnaissance sampling led to the classification of MORB into broad petrographic provinces, with particular emphasis on compositional differences between MORB recovered near hot spots and those recovered far from hot spots, as well as differences among hot spots [e.g.,
Miyashiro et al., 1969; Melson and O'Hearn, 1979; Morel and Hekinian, 1980; Sigurdsson, 1981; Schilling et al., 1983; Dmitriev et al., 1984]. With further sampling and study, differences in parental magma composition were recognized even among basalts recovered far from hot spots [e.g., Langmuir and Hanson, 1981; Bryan and Dick, 1982; Langmuir and Bender, 1984]. Dick et al. [1984] examined basalts from thirteen ridge segments in the Atlantic and Indian Oceans and suggested that they exhibited regional differences in parental magma composition as a result of variations in the extent of melting.

$K L 87$ extended the investigation of global systematics of major element variations in basalts to include all ridge segments for which data were available as of about 1986 . $K L 87$ normalized the $\mathrm{Na}_{2} \mathrm{O}$ and $\mathrm{FeO}$ data to a constant value of $\mathrm{MgO}$ in order to correct for low-pressure fractionation, as described above, and averaged the data from each region. They found that regional averages of $\mathrm{Na}_{8.0}, \mathrm{Fe}_{8.0}$ and $\mathrm{CaO} / \mathrm{Al}_{2} \mathrm{O}_{3}$ correlated with the mean axial depth of the ridge, with the exception of data for samples collected very close to some hot spots. Application of interlaboratory corrections to the global data set and the availability of some new data clarifies important aspects of the global correlations (Figure 15). Each data point is an average of samples from approximately 100 $\mathrm{km}$ of ridge length. Although all data points together exhibit rough correlations between the chemical parameters and axial depth, it is clear from the figure that a useful separation can be made between (1) basalts from all normal ridges; (2) basalts from back-arc basins; and (3) basalts from ridge segments that have been influenced by certain hot spots (Azores, Jan Mayen, Bouvet, Tristan/Gough, Galapagos). For the normal ridges, $\mathrm{Na}_{8.0}, \mathrm{Fe}_{8.0}$ and axial depth correlate strongly. We call these relationships the "global correlations" since they apply to the global system of ridges that have not been substantially influenced by hot spots. Back-arc basins as a group also show correlations among $\mathrm{Fe}_{8.0}, \mathrm{Na}_{8.0}$ and depth, but the data are systematically offset to lower $\mathrm{Fe}_{8.0}$ in comparison to normal ridges. Some ridges influenced by hot spots have anomalous major element compositions in comparison to normal ridges (i.e., low $\mathrm{Fe}_{8.0}$ and sometime $\mathrm{Na}_{8.0}$ ). For these ridges, there are also different relationships between chemistry and axial depth than normal ocean ridges, as discussed further below.

Careful examination of the regionally averaged data reveals finer distinctions within the global correlations. Basalts from the Indian Ocean have systematically lower $\mathrm{Fe}_{8.0}$ (Figure 16a) and higher $\mathrm{Na}_{8.0}$ than basalts from the Atlantic for a given depth. Normal sections of the EPR and the northern MAR are distinct-- basalts from the MAR between $23^{\circ}$ and $32^{\circ} \mathrm{N}$ are high in $\mathrm{Fe}_{8.0}$ for a given $\mathrm{Na}_{8.0}$ (Figure $16 b$ ). It appears that substantial portions of the ocean ridge system have their own chemical signature, which is a cause of the width of the overall field of the global correlations.

The discussion above shows that the effects of hot spots, back-arc basins and regional variability lead to noise in the 

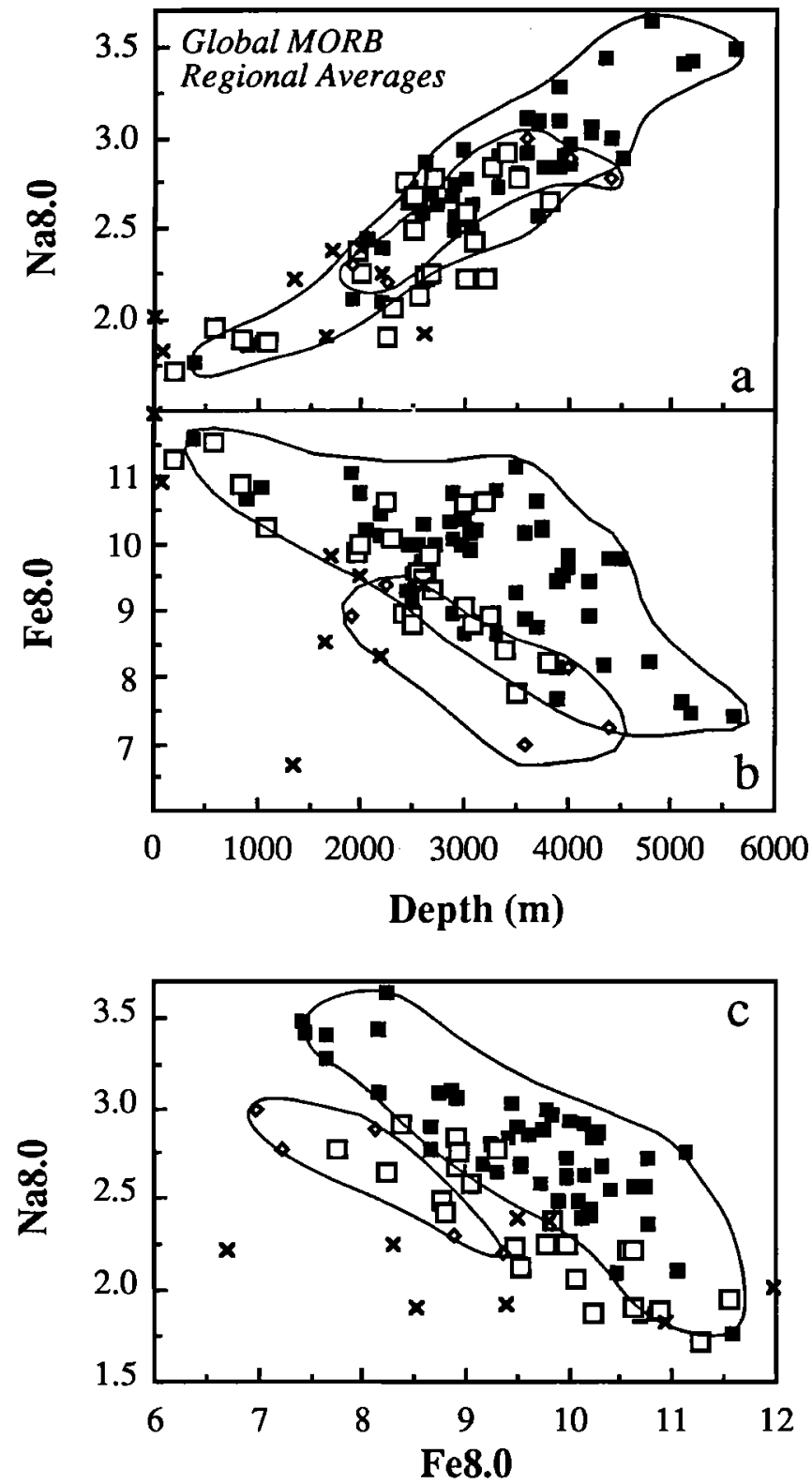

Fig. 15. Global correlations between regional averages of smoothed axial depth and the $\mathrm{Na}_{8.0}$ and $\mathrm{Fe}_{8.0}$ of MORBs. This figure is the same as Figure 2 in $K L 87$, except all analyses have been recalculated to sum to $100 \%$ (all iron as $\mathrm{FeO}$ ) and have been corrected for interlaboratory differences. Corrections were applied to all analyses determined at the Smithsonian Institution [Melson et al. 1977] or that were reported to be consistent with the Smithsonian data [i.e., Schilling et al., 1985], based on the factors reported in $K L 89$ (their Figure 5), so that all data are consistent with those determined at Lamont and by wet chemistry at URI. Data sources as in $K L 87$, with updates from the most recent Smithsonian Catalogue of Basalt Glasses [Mulson, pers. comm.] Batiza et al. [1988], Fryer et al. [1990], and LDGO unpublished data from $23-32^{\circ} \mathrm{N}$ on the MAR. Different groups of MORB are distinguished: solid boxes are MORB from "nornal" ridge segments; diamonds are from back-arc basins; open squ ures are from ridges influenced by the Galapagos, Azores, Jan Mayen, Tristan, Iceland, and Bouvet hot spots; Xs are from ridge segments inim, ediately adjacent to these hot spots. Note that Iceland and adja :ent ridges are also coincident with the normal MORB array. global data set, but that global systematics emerge for different subsets of the data. For $\mathrm{TiO}_{2}$, the regional effects are even more pronounced, so that the data set as a whole does not show obvious systematic relationships with other chemical parameters or with depth (Figure 17). However, separation of the data into different groups does clarify the $\mathrm{Ti}_{8.0}$ observations. Regional averages of $\mathrm{Ti}_{8.0}$ for normal ridges exhibit a positive correlation with $\mathrm{Na}_{8,0}$, and hence with depth. Ridges influenced by hot spots show variable behavior. Basalts from Iceland are very high in $\mathrm{TiO}_{2}$, which is characteristic of many hot spots world wide [e.g., Feigenson et al., 1983; Albarede and Tamagnan, 1988]. Some other hot spot-influenced ridges (e.g. MAR near the Azores), however, have lower $\mathrm{TiO}_{2}$ than normal ridges. Back-arc basins are all
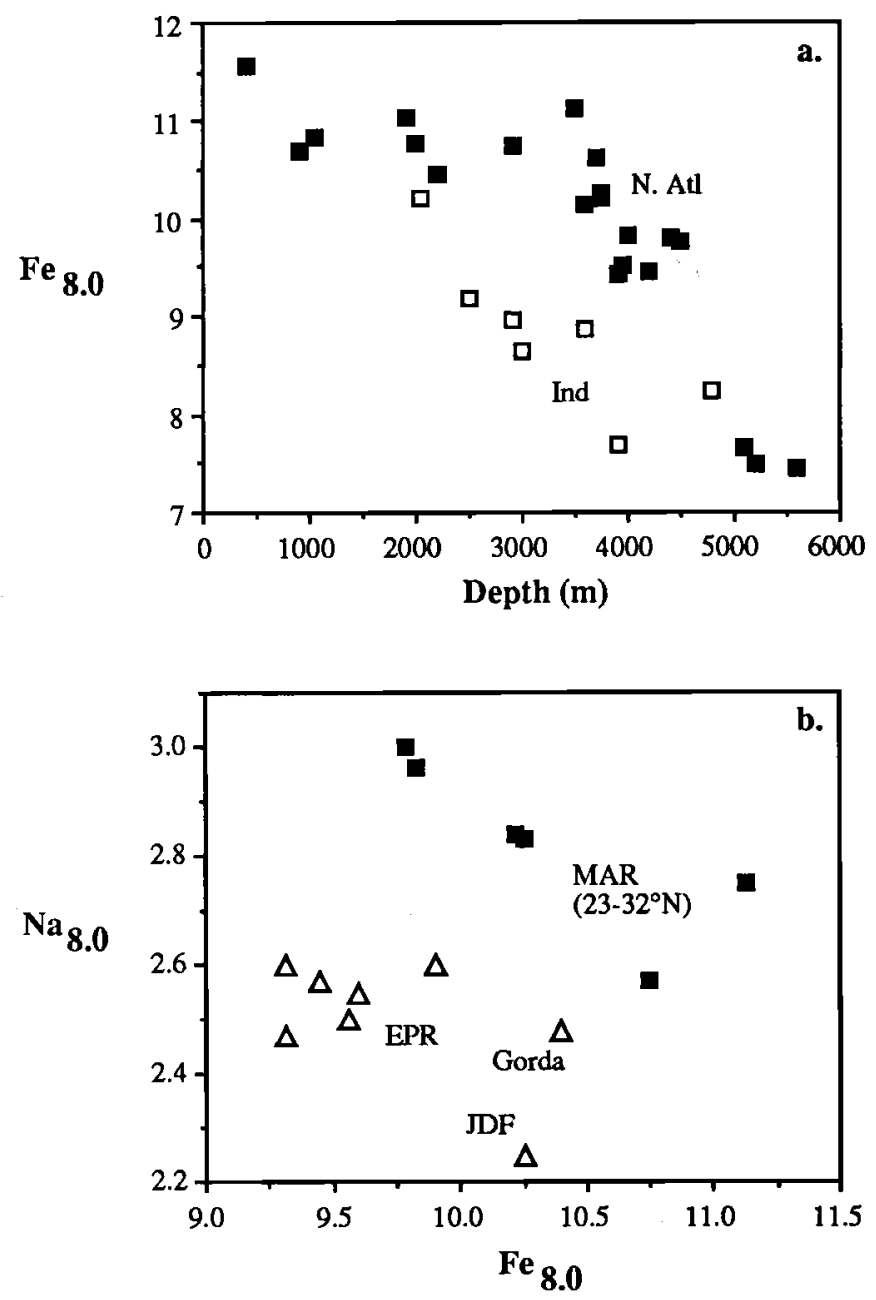

Fig. 16. Systematics within the "normal" ridge data for different ocean basins, as in Figure 15 , a) Indian Ocean MOP B are off-set to lower $\mathrm{Fe}_{80}$ (shown) and higher $\mathrm{Na}_{\mathrm{g}, 0}$ (not shown) than N. Atlantic MORB for the same axial depth $b$ ) There is a systematic offset between $\mathrm{N}$. Atlantic and Pacific MORB. These subtle inter-ocean differences contribute to the observed "width" of the global correlations for normal ridges of Figure 15. 


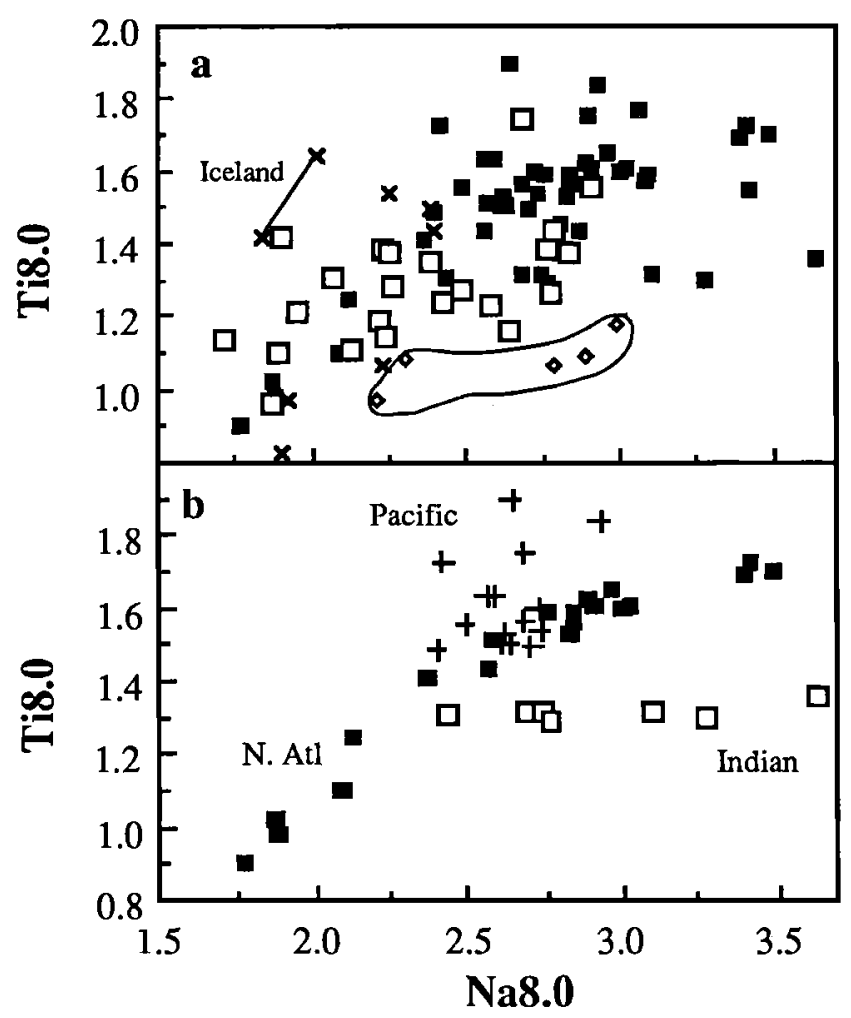

Fig. 17. Regional averages for $\mathrm{Ti}_{8.0}$ in MORB. a) Global variations in MORB. Symbols as in Figure 15. o) Systematics within the "normal" MORB data for different ocean basink. Data sources as in Figure 15 . $\mathrm{Ti}_{8.0}$ calculated for samples with $5-8.5 \mathrm{wt} \% \mathrm{MgO}$ from: $\mathrm{Ti}_{8.0}=\mathrm{TiO}_{2}+0.351(\mathrm{MgO}-8)$.

low in $\mathrm{TiO}_{2}$. Within the normal ridge population, MORB from Indian Ocean Ridges are low in $\mathrm{TiO}_{2}$, and MORB from the Pacific are slightly high in $\mathrm{TiO}_{2}$ (Figure $17 b$ ). $\mathrm{Ti}_{8.0}$ in basalts from the North Atlantic correlates very well with both depth and $\mathrm{Na}_{8.0}$. Thus $\mathrm{Ti}_{8.0}$ appears to show similar global systematics to $\mathrm{Na}_{8.0}$, but is strongly affected by the differences among different hot spots, by the back-arc environment, and by regional differences.

One of the most important aspects of the global correlations for normal ridges (Figure 15) is that they relate the composition of the ocean crust to a fundamental physical characteristic of the ridge: axial depth. In addition, $K L 87$ showed that $\mathrm{Na}_{8.0}$ correlates roughly with crustal thickness, a result that remains with the inclusion of more recent data (Figure 18). The relationship with crustal thickness shown in Figure 18 relies to some extent on Iceland and the Mid-Cayman rise (the thick and thin end-members), and the Mid-Cayman Rise crustal thickness value is inferred from geologic rather than seismic constraints [Stroup and Fox, 1981]. However, seismic data from the northern part of the Kolbeinsey Ridge [Evans and Sacks, 1979; unintentionally omitted from the compilation in $K L 87$ ] suggest a crustal thickness of $15 \mathrm{~km}$, confirming the association of low $\mathrm{Na}_{8.0}$ values with anomalously thick crust, and data from the Arctic Ridge suggests the association of very thin crust with deep ridges.

In order to better fully characterize the relationship between crustal chemistry and crustal thickness, it is important that crustal thicknesses be determined in other regions, particularly those that are anomalously shallow or deep. One needs to bear in mind, however, the intrinsic limitations of seismic measurements of "crustal thickness." Ultzamafic cumulates are petrologically part of the crust, and yet they may have high seismic velocities similar to mantle values. Crust with a significant thickness of such cumulates may thus appear anomalously thin seismically. There is also evidence in the Atlantic for substantial disruption of crust, and the presence of abundant serpentinized peridotite at high crustal levels [e.g., Karson et al., 1987; Bougault, personal communication]. In this case, seismic "crustal thickness" may reflect tectonic disturbance or depth of hydrothermal alteration rather than the volume of magma derived from the mantle.

\subsection{Regional Variability Around Hot Spots}

Certain hot spots influence the chemical composition of ridges so that they do not show the same relationship between

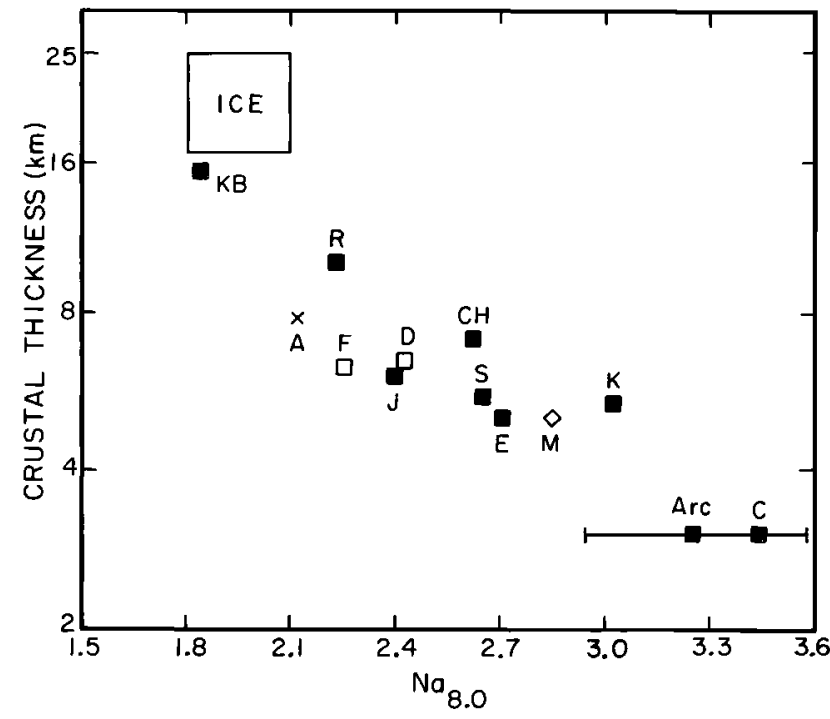

Fig. 18. Regional averages of $\mathrm{Na}_{8.0}$ vs. crustal thickness. Data and references are the same as in Klein and Langmuir [1987] (their Figure 14) except Smithsonian data has been corrected for interlaboratory bias as in Figure 14 and new data points have betn added. Kolbeinsey crustal thickness is from Evans and Sacks [1979]. The crustal thickness for the Reykjanes Ridge has been increased to $10 \mathrm{~km}$ based on considerations of the off-axis profile of Bunch and Kennett [1980], since this reflects fully mature crust. For the Arctic Ridge, there is a crustal thickness estimate [Reid and Jackson, 1981; Jackson and Reid, 1980] but no $\mathrm{Na}_{8,0}$ data. $\mathrm{Na}_{8.0}$ is estimated from tie global $\mathrm{Na}_{8.0}$-depth correlation (Figure 15). The error bar on the point represents the variation in $\mathrm{Na}_{8.0}$ that would be possible across the entire global array for a mean depth for the Arctic Ridge of $4500 \mathrm{~m}$ [IIIO/IOC/CHS, 1984]. Other regions shown are I (Iceland), R (Reykjaniss Ridge), F (MAR at $37^{\circ} \mathrm{N}$ ), A (Azores region), D (MAR at $45^{\circ} \mathrm{N}$ ), J (Juan de Fuca), S (EPR near Siqueiros transform), E (East Pacific Rise), K (MAR, Kane transform), M (Mariana Trough), $\mathrm{CH}\left(5-14^{\circ} \mathrm{N}\right.$ on EPR), KB (Kolbeinsey Ridge), Arc (Arctic ridge), C (Cayman Trough). 
chemistry and axial depth as normal ocean ridges. This is apparent for MORB from the Galapagos platform (Figure 19). Note that these data are for individual locations rather than the regional averages discussed above. $\mathrm{Na}_{8.0}$ increases, $\mathrm{Fe}_{8.0}$ decreases, and depth shallows systematically towards the center of the hot spot. $\mathrm{Na}_{8.0}$ and $\mathrm{Fe}_{8.0}$ correlate negatively with a similiar slope to the global variability, but the relationship with depth is the opposite of that observed globally. The shallowest regions have the lowest $\mathrm{Fe}_{8.0}$ and the highest $\mathrm{Na}_{8.0}$ (Figure 19).

Not all hot spots, however, exhibit this anomalous relationship. For data from the Reykjanes Ridge, south of
Iceland (Figure 20), $\mathrm{Na}_{8.0}$ decreases and $\mathrm{Fe}_{8.0}$ increases as the depth shallows. This is consistent with the overall global relationships among these parameters, although $\mathrm{Na}_{8.0}$ may increase slightly over the Iceland hot spot.

As pointed out above, $\mathrm{TiO}_{2}$ may also show a distinctive hot spot effect. The positive correlation between $\mathrm{Ti}_{8.0}$ and $\mathrm{Na}_{8.0}$ for normal ridges may breakdown near high- $\mathrm{TiO}_{2}$ hot spots such as Iceland. Indeed, basalts from Iceland and the northern Reykjanes Ridge have among the highest $\mathrm{TiO}_{2}$ and lowest $\mathrm{Na}_{8.0}$ of any MORBs (Figure 17).

These observations from near hot spots indicate that major element chemical effects associated with some hot spots are

\section{Galapagos Spreading Center}
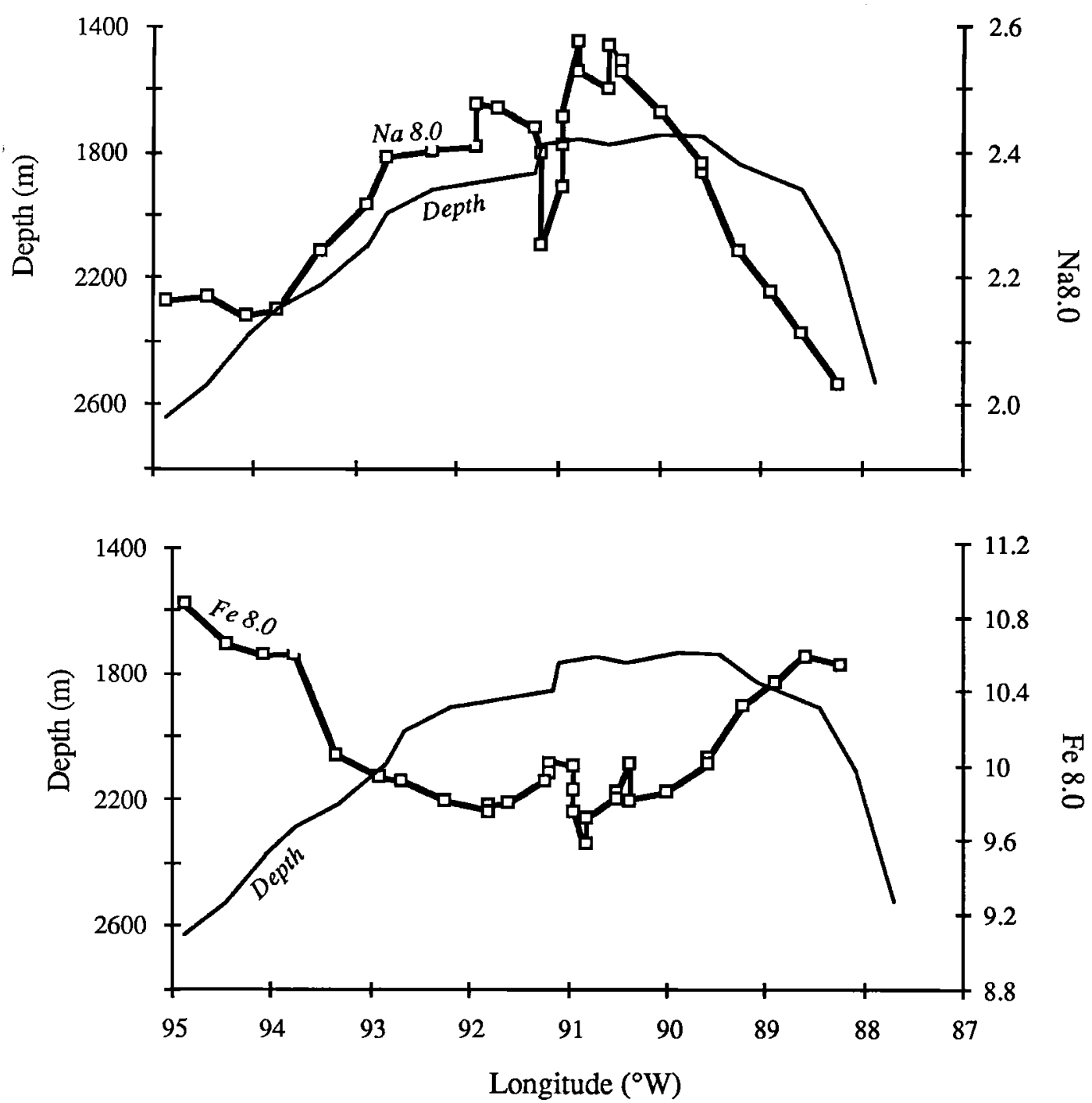

Fig. 19. Variations in basalt composition and axial depth across the Galapagos platform. Microprobe data from Fisk et al. [1982] and depths from Schilling et al. [1982]. Points plotted are five-point running averages of the data, in order to give a regional perspective and smooth out the smallest scale noise from individual dredges. The Galapagos Islands themselves are between $90^{\circ} \mathrm{W}$ and $92^{\circ} \mathrm{W}$. 

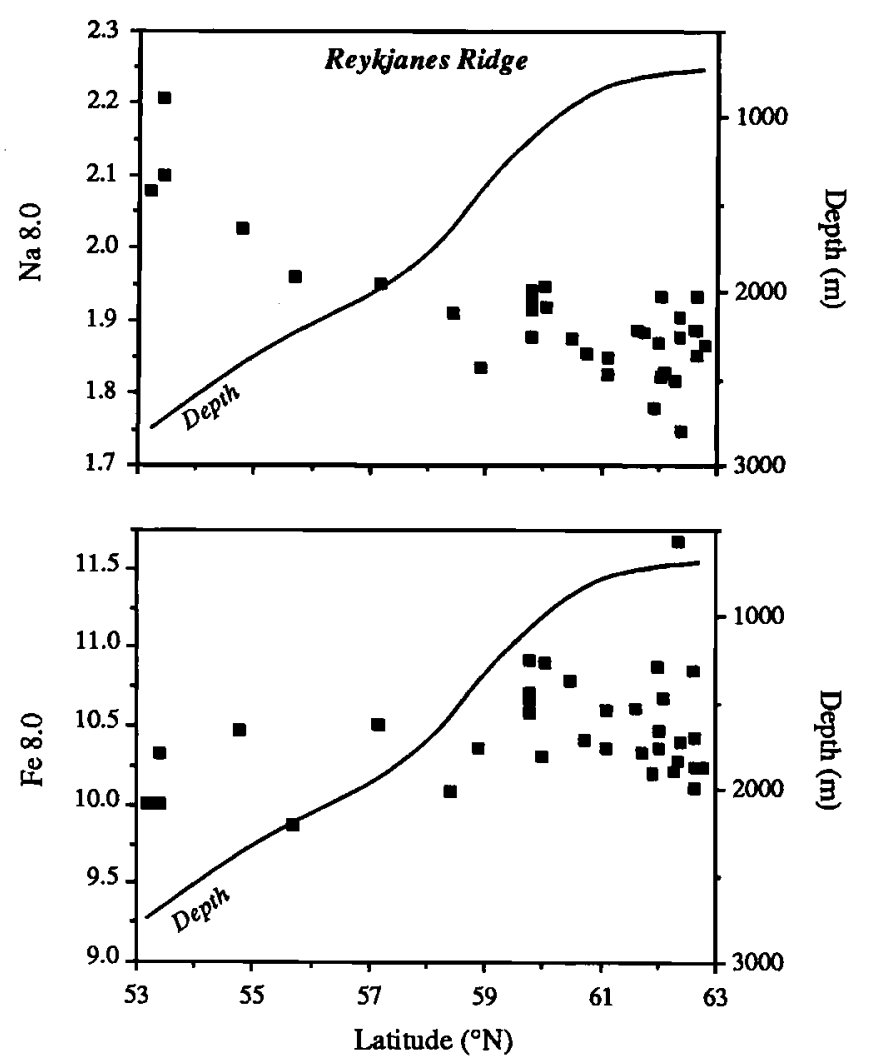

Fig. 20. Variations in basalt composition and axial depth along the Reykjanes Ridge. Each point is for a single sa nple group within a dredge. Microprobe data from Melson et al. [1977 and pers. comm.]. Depth curve is from dredge depths. The Reykjanes Ridge extends onto Iceland at about $63.8^{\circ} \mathrm{N}$.

distinct from those exhibited in other regions, and therefore a single explanation cannot apply to the major element systematics of all MORB. It is also clear that different hot spots (e.g., Iceland and the Azores lead to distinct major element effects on MORB. This latter point has been emphasized previously by Langmuir and Hanson [1980] and Schilling et al. [1982].

\subsection{Local Variability of Major Elements in MORB}

Above, we discussed the systematic global variability in average MORB chemistry over distances of hundreds to thousands of kilometers. Looking at the data from a global point of view is a comparatively recent development, because it has only been in recent years that enough high-quality data have been collected from diverse ridge segments. This global coverage is made up of a large number of investigations, representing more than two decades of studies of the local compositional variations among MORB recovered from individual ridge segments [e.g. Muir et al., 1964]. Such studies have identified significant local chemical systematics for both major and trace elements. This chemical variability has been attributed to both magma chamber [Bryan and Moore, 1977;
Dungan and Rhodes, 1978] and melting processes [White and Bryan, 1977; Langmuir et al., 1977], although the evidence for variations in the extent of melting has in the past relied almost exclusively on trace element modeling.

Klein and Langmuir [1989; hereafter KL89], prompted by the work of Brodholt and Batiza [1989], explored the nature of local chemical variability and how it compares to global variability, using the fractionation-corrected parameters of $K L 87$. Examining data from densely sampled ridge segments, $K L 89$ found that there were systematic local chemical variations, and that these systematics can differ substantially from the global vector of variability. $K L 89$ emphasized data from the Atlantic, but noted that the systematics for the East Pacific Rise (EPR) and the mid-Atlantic ridge (MAR) were very different. Here we present the nature of the local variability for the MAR and EPR, which may be representative of slow- and fast-spreading ridges, respectively.

\subsubsection{Local Variability on Slow-Spreading Ridges}

Figure 21 shows $\mathrm{Na}_{8.0}$,vs. $\mathrm{Fe}_{8.0}$ for densely sampled segments of slow-spreading ridges (mostly in the North Atlantic). Local trends from "normal" ridges are compared to the "normal" global trend (Figure 21a) and local trends from ridges near hot spots are compared to the global trend for hot spot-influenced regions (Figure $21 b$ ). The data from each individual region form short, sub-parallel trends stacked in the direction of the global vector, but the trends for each local region are oblique to the global trend on each plot. Particularly striking are the data from the section of the MAR between the Kane and Hayes Fracture Zones (Figure 21a). In this region, erupted basalts are normal MORB, yet the ridge depth varies by 1500 meters. Thus this region provides a natural experiment of the relationship between chemistry and depth independent of the anomalous effects produced by hot spots. Averages over long distances $(100 \mathrm{~km})$ from this region are consistent with the global correlations, and yet the local variability over short distances is of the opposite slope to the global variation on plots of $\mathrm{Na}_{\mathbf{8 . 0}}$ vs. $\mathrm{Fe}_{\mathbf{8 . 0}}$. Thus the local chemical variability has its own chemical systematics which are distinct from the global systematics among regional averases.

It is significant that a clear local vector also occurs for one of the most enriched portions of the ocean ridge system, near the Oceanographer Fracture Zone (Figure 21b). In this region, several mantle components have been identified on the basis of radiogenic isotope data, and very large variations exist in the REE patterns of erupted basalts [Shirey et al., 1987]. Within each compositional group, the data exhibit clear positive correlations between $\mathrm{Na}_{8.0}$ and $\mathrm{Fe}_{8.0}$. This region, therefore, would potentially allow a clear distinction to be made between the processes causing the local vector and the effects of heterogenerous components in the mantle.

These observed chemical systematics appear to reflect processes that can occur within or beneath individual ridge segments, and will be referred to as the "local" correlations. Recall that in our discussion of the variations observed on $\mathrm{MgO}-\mathrm{Na}_{2} \mathrm{O}$ diagrams (Figure 12 ), we referred to local variability 


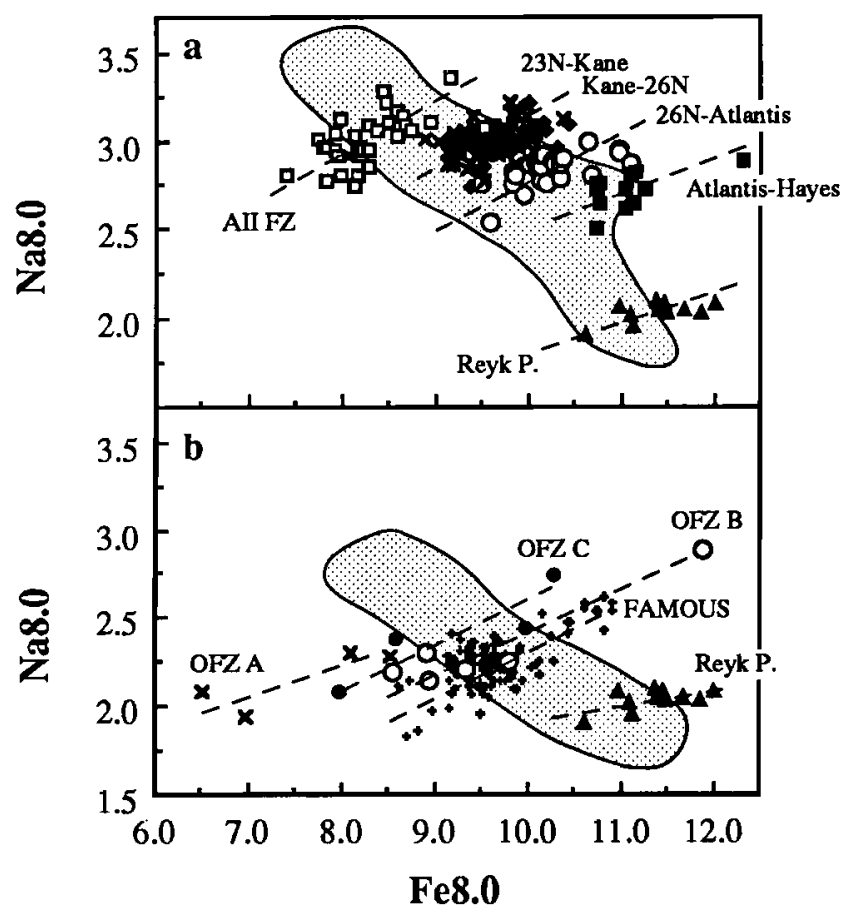

Fig. 21. $\mathrm{Fe}_{80}$ vs. $\mathrm{Na}_{30}$ for individual samples from various densely sampled ridge segments. a) Samples from "nomal" ridge segments. Shaded field is for regional averages of data from "normal" ocean ridges from Figure 15. Solid triangles for Reykjanes Peninsula [Jakobsson et al., 1978]; Xs for $23^{\circ} \mathrm{N}$ to the Kane Fracture Zone [W. Melson, pers. comm.]; filled diamonds from the Mid-Atlantic Ridge $23^{\circ} \mathrm{N}-26^{\circ} \mathrm{N}$ (Langmuir, unpublished data); open circles from $26^{\circ} \mathrm{N}$-Atlantis FZ (Langmuir, unpublished data); filled squares from Atlantis FZ to Hayes FZ (Langmuir, unpublished data) ; open squares for Atlantis II FZ [Johnson and Dick, 1992]. Note how Atlantic data are offset to the high side of the normal field, while the Indian data are on the low side (see Figure 16). b) Samples from ridge segments influenced by hot spots, compared to shaded field for regional averages of hot spotinfluenced ridges from Figure 15. Solid triangles for Reykjanes Peninsula as above; plusses for FAMOUS and AMAR regions of the MAR [W. Melson, pers. comm.]. Samples from near the Oceanographer Fracture Zone (OFZ) [Bender and Langmuir, unpublished data] are in three groups based on $\mathrm{Ce} / \mathrm{Yb}$ as follows [J. Bender, unpublished isotope dilution data; Shirey et al., 1987]: $\mathrm{Ce} / \mathrm{Yb}=9.7$ 10.7 for $A,=7.4-9.1$ for $B,=4.2-6.5$ for $C$. (Note that groupings $A-C$ here are not the same as the A-B-C groups of Shirey et al. [1987]). All data have been renormalized to $100 \%$, and corrected for interlaboratory biases as for Figure 15. The $\mathrm{Na}_{8.0}$ and $\mathrm{Fe}_{8.0}$ algorithms for samples south of Kane and the Reykjanes Peninsula have been adjusted as in Klein and Langmuir [1989], their Figure 5.

as the "scatter" in the data from an individual region at a constant value of $\mathrm{MgO}$, that could not be explained by lowpressure crystal fractionation. Our examination of local variability here shows that there is fine-structure within the scatter of the data from individual regions that is revealed when the effects of fractionation are removed.

The local correlations are also not artifacts of the fractionation-correction applied to the data. This can be demonstrated by examining a region for which a large number of samples exist. The FAMOUS/AMAR region of the MidAtlantic Ridge is among the most densely sampled segments of the ocean ridge system, and spans less than $50 \mathrm{~km}$ of ridge length. In the Smithsonian data file, there are over 160 individual analyses from this region (Figure 22a). The 62 analyses that fall within the narrow range of $8.0+/-0.25 \mathrm{wt} \%$ $\mathrm{MgO}$ (Figure $22 b$ ) show no obvious correlations between $\mathrm{Na}_{2} \mathrm{O}$ with $\mathrm{MgO}$, yet $\mathrm{Na}_{2} \mathrm{O}$ contents range from $1.85 \mathrm{wt} \%$ to 2.45 wt\%. These samples do exhibit systematic variations between $\mathrm{Na}_{2} \mathrm{O}$ and other major and minor elements (Figure 23). The trends in $\mathrm{Na}_{2} \mathrm{O}, \mathrm{FeO}$ and $\mathrm{SiO}_{2}$ shown in Figure 23 are similar in slope and magnitude to the $\mathrm{Na}_{8.0}, \mathrm{Fe}_{8.0}$ and $\mathrm{Si}_{8.0}$ variations for this region shown by $K L 89$, although they do not span as great a range in values. Furthermore, although the local trends were originally recognized by $K L 89$ on the basis of $\mathrm{Na}, \mathrm{Fe}$ and $\mathrm{Si}$ covariations, other significant correlations also exist between $\mathrm{Na}_{2} \mathrm{O}$ and $\mathrm{CaO}, \mathrm{TiO}_{2}$ and $\mathrm{K}_{2} \mathrm{O}$, but not $\mathrm{Al}_{2} \mathrm{O}_{3}$ (Figure 23). Thus, it is clear from the FAMOUS/AMAR data that the Atlantic-type local trends occur not only in samples corrected to $8 \mathrm{wt} \% \mathrm{MgO}$ but also in the raw data for samples with approximately $8 \mathrm{wt} \%$

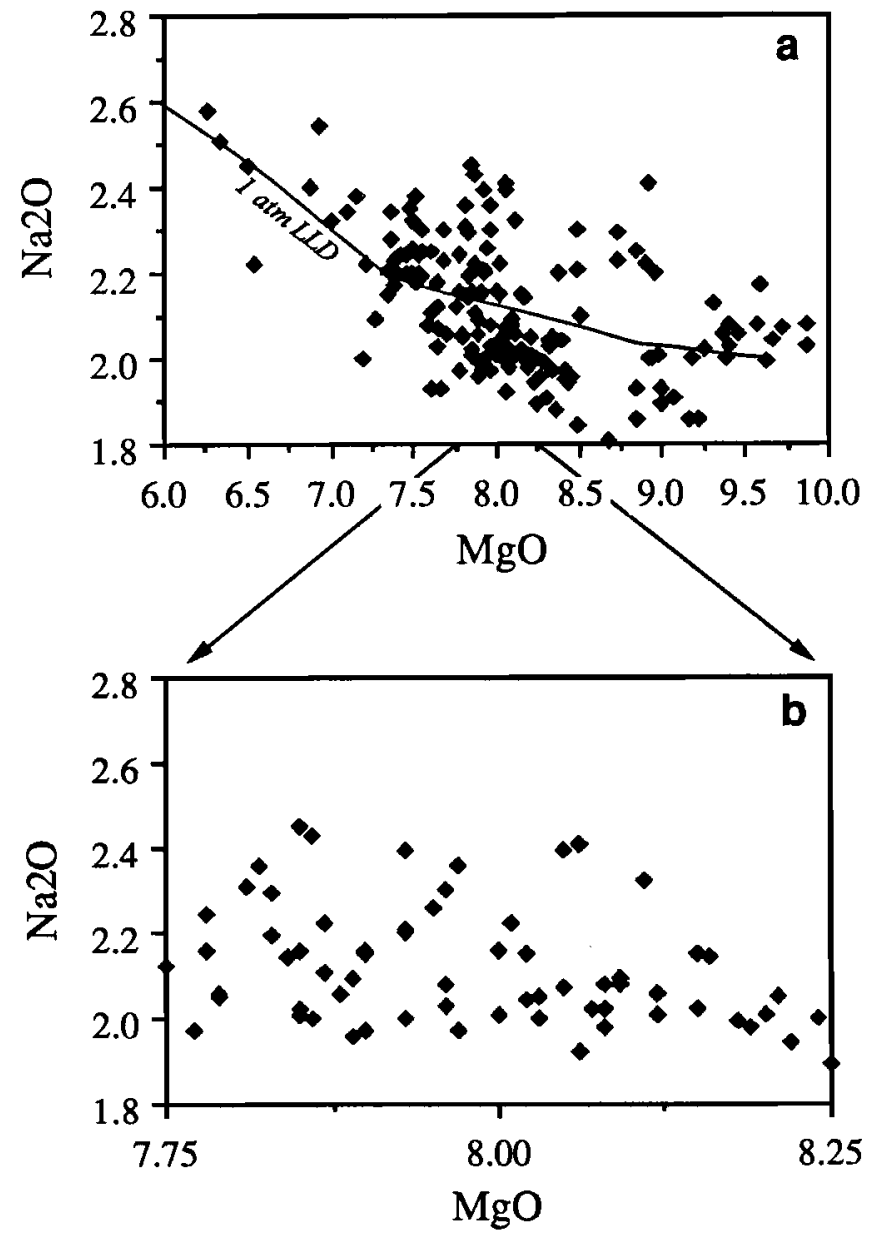

Fig. 22. Illustration of local variability for well-sampled ridge segment. $\mathrm{MgO}$ vs. $\mathrm{Na}_{2} \mathrm{O}$ for $a$ ) all samples from the FAMOUS/AMAR region of the MAR included in the Smithsonian Catalogue of Basal Glasses [Melson et al., 1977; and pers. comm /, and b) only those analyses with $7.75-8.25$ wt\% $\mathrm{MgO}$. Data have toen re-normalized to $100 \%$, but interlaboratory correction factors have not been applied. 

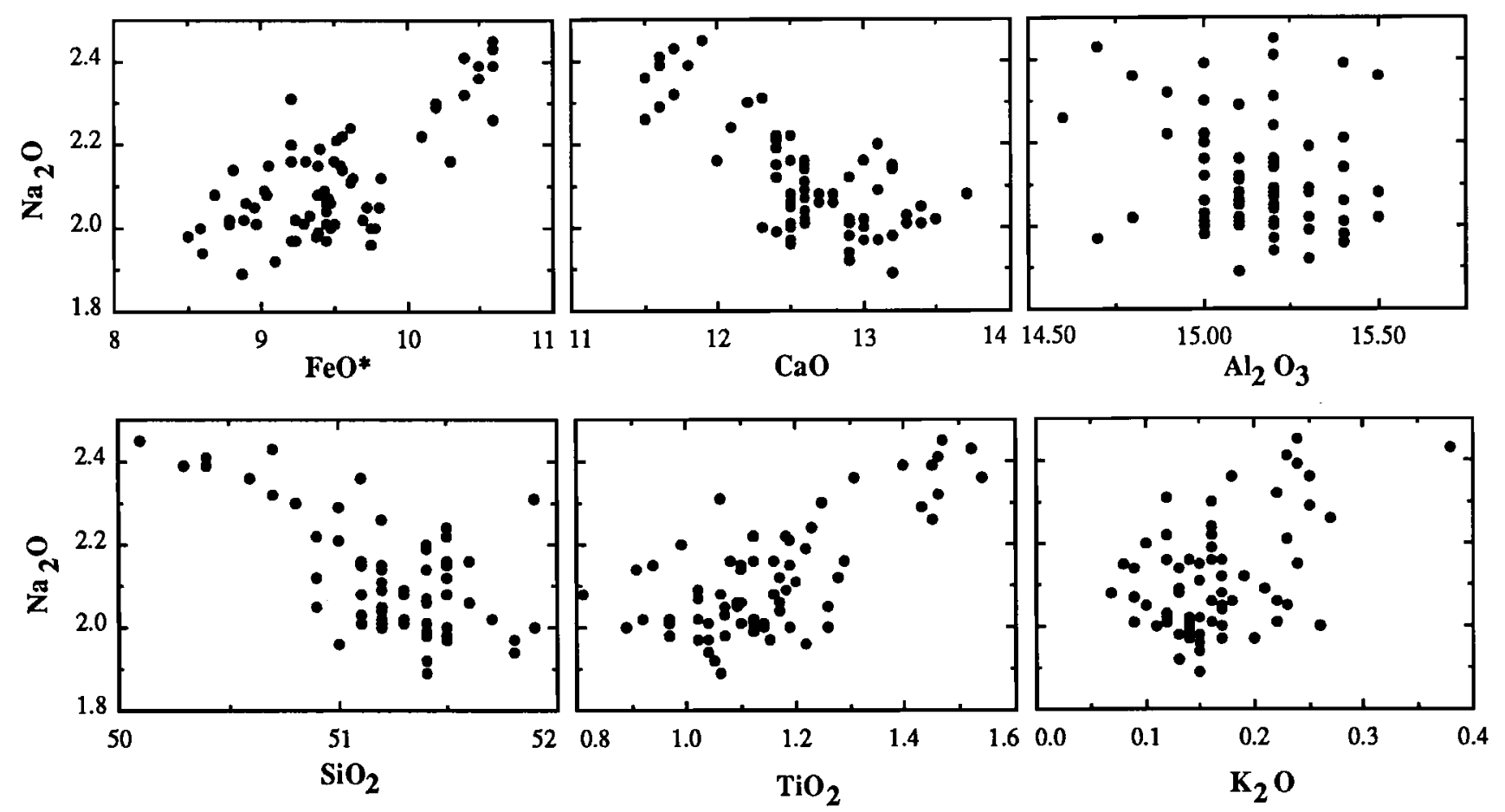

Fig. 23. Local variability for a narrow window of $\mathrm{MgO}$, in order to remove all errors that could be associated with fractionation correction, using samples from FAMOUS/AMAR region with $7.75-8.25 \mathrm{wt} \% \mathrm{MgO}$, as in Figure $22 b$. Note that most elements show systematic correlations with one another even at constant $\mathrm{MgO}$ content.

$\mathrm{MgO}$. This conceptually simplifies the problem of explaining the origin of these local trends; we simply need to identify processes that produce the observed variability at $8 \mathrm{wt} \% \mathrm{MgO}$.

The fact that clear local vectors occur for the FAMOUS/AMAR region, with transitional trace element chemistry characteristic of a hot spot margin, for the Oceanographer region, which is one of the most enriched parts of the ocean floor, and for the Kane to Hayes region, which is of normal MORB composition, shows that the local chemical systematics are not controlled by mantle heterogeneity or other effects that contribute to hot spots. Instead, it appears that Atlantic local variability is characteristic of the slow spreading ridge environment. Similar local vectors have recently also been found on the very deep and slow-spreading Southwest Indian Ridge by Johnson and Dick [1992] (Figure 21 ), so that these local trends occur over the entire range of axial depth and $\mathrm{Na}_{8.0}$ values at slow spreading ridges.

\subsubsection{Local Variability on the East Pacific Rise}

The data from the fast-spreading East Pacific Rise (EPR) have very different systematics than those observed at slowspreading ridges. In order to present the rariability along the EPR, we have normalized the data to the nean $\mathrm{MgO}$ value for a large number of EPR samples (7.3 wt\% $\mathrm{MgO})$, because the EPR contains few samples with greater than 8 wt\% MgO. Choosing this normalization value ensures that the fractionation corrections themselves have minimal influence on the distribution of the normalized data.

The EPR shows much less depth variation than the MAR, over both short and long distances along the axis. Nonetheless, individual EPR basalts have a large range in composition after correction for fractionation. The $\mathrm{Na}_{7.3}-\mathrm{Fe}_{7.3}$ variations for EPR data sets are parallel to the global vector, and the range for individual samples (Figure 24) is almost as large as the global range for regional averages! Note, however, that average EPR data plots in the middle of the global field which corresponds with its typical depth of about $2700 \mathrm{~m}$ (see Figure 15).

This variability for the EPR as a whole is in fact a form of local variability because it can occur between small ridge offsets along as little as $50 \mathrm{~km}$ of the axis. Figure $24 \mathrm{~b}$ compares data from about $50 \mathrm{~km}$ of the EPR north of $12^{\circ} \mathrm{N}$ [Langmuir et al., 1986; Reynolds et al., 1992 in press] with the entire EPR from $5-14^{\circ} \mathrm{N}$ and shows that there is virtually complete overlap. Within this $50 \mathrm{~km}$ region there are no major ridge offsets and only a $100 \mathrm{~m}$ change in axial depth, so the chemical variations clearly occur on a local scale, even in the absence of significant disturbances of the crust or thermal perturbations of the mantle. The local EPR variability is similar to the total range of EPR data partly because the regional depth along the EPR varies little.

From the $\mathrm{Na}_{8.0}-\mathrm{Fe}_{8.0}$ perspective, there is striking overlap between the EPR local and global avaraged data. But the 


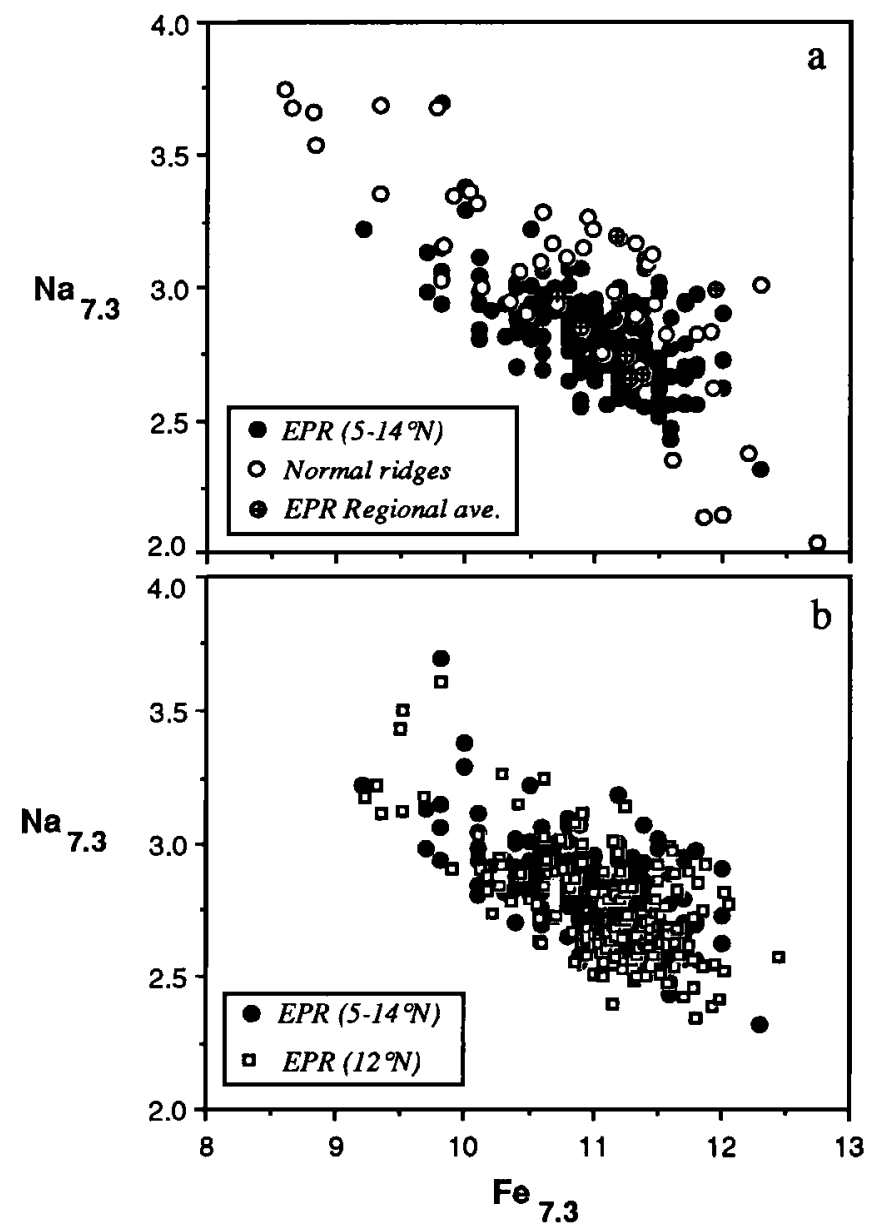

Fig. 24. $\mathrm{Na}_{73}$ vs. $\mathrm{Fe}_{7}$ for individual samples from $5-14^{\circ} \mathrm{N}$ on the East Pacific Rise (EPR) from the CHEPR expedition [microprobe analyses of glasses, $J$. F. Bender and $C$. H. Langmuir, unpublished data] compared to a) global regional averages for normal ocean ridges and EPR averages (from Figure 15), and $b$ ) individual samples from $12^{\circ} \mathrm{N}$ on the EPR from the VENTURE expedition [microprobe analyses of glasses, Reynolds et al., 1992 and unpublished data]. Compositions are compared at $7.3 \mathrm{wt} \% \mathrm{MgO}$ instead of $8 \mathrm{wt} \% \mathrm{MgO}$ because $7.3 \%$ is the mean for all EPR basalts. Although the $\mathrm{Na}$-Fe variations of EPR and the global vector are similar, this similarity does not extend to other elements.

similarity ends there. The systematics of the EPR data are not similar to the global vector for all elements. Plots of $\mathrm{Na}_{7.3} \mathrm{vs}$. $\mathrm{Si}_{7.3}$ and $\mathrm{Fe}_{7.3}$ vs. $\mathrm{Si}_{7.3}$ do not exhibit the characteristic slopes of the global data (Figure 25, see also KL89). In fact, some individual areas show weak positive correlations between $\mathrm{Fe}_{7.3}$ and $\mathrm{Si}_{7,3}$. There are a few samples from the EPR which have high $\mathrm{Na}_{7.3}$ and low $\mathrm{Si}_{7.3}$, creating one sparse limb of the EPR field that is parallel to the Atlantic data. Yet these same samples have low $\mathrm{Fe}_{7.3}$, which is opposite to the MAR local vector. The characteristic inverse relationship between $\mathrm{Fe}$ and $\mathrm{Si}$ that is present for the global correlations and the slowspreading local vector is completely absent in the EPR data. Therefore the EPR local systematics are distinct from both the global vector and the Atlantic local vector.
Two components of EPR local variability. Examination of the other major elements suggests there may be two components of local variability in the EPR data, one within "normal" MORB (N-MORB, with low $\mathrm{K}_{2} \mathrm{O} / \mathrm{TiO}_{2}$ ), and one between N-MORB and "transitional" MORB (T-MORB, with higher $\mathrm{K}$ contents). In the sample group as a whole, plots of many of the major elements show scatter (Figure 26), until the N-MORB are separated from the T-NOR3. Reynolds et al. [1992, in press] have pointed out that $\mathrm{Fe}_{7.3}$ and $\mathrm{Ti}_{7.3}$ correlate positively for the N-MORB alone in the $12^{\circ} \mathrm{N}$ region, and that temporal variations emerge much more clearly from consideration of the N-MORB alone.

This variation for the N-MORB, however, does not produce the striking negative $\mathrm{Na}_{7.3}-\mathrm{Fe}_{7.3}$ correlation for the EPR, because the $\mathrm{N}$-MORB alone exhibit little variation in $\mathrm{Na}_{7.3}$. Instead, the range in $\mathrm{Na}_{7.3}$ results from a different compositional vector, that extends from N-MORB to T-MORB. The high $\mathrm{K}_{2} \mathrm{O}$, T-MORB component has low $\mathrm{FeO}$ and high $\mathrm{Na}_{2} \mathrm{O}$, and also high $\mathrm{TiO}_{2}$, low $\mathrm{SiO}_{2}$, and high $\mathrm{Al}_{2} \mathrm{O}_{3}$. These characteristics of the transitional component correlate quantitatively. For example, the excess $\mathrm{TiO}_{2}$ (defined as the $\mathrm{Ti}$ in excess of the N-MORB Fe-Ti correlation line shown in Figure 26) correlates well with most of the major elements, as illustrated in Figure 27. The transitional component is slightly variable along the EPR, leading to local arrays that are slightly offset from one another for some elements.

Thus the Pacific local variability requires two compositional components. The component of variability that occurs within $\mathrm{N}$-MORB has little variation in $\mathrm{Na}_{7.3}$, and limited variation in many other elements. The substantial variability that occurs in EPR lavas results from a second chemical component that is related to the variable presence of "enriched" material. The major chemical variations correlate with the amount of this enriched component. This is apparent in Figure 28, where it can be seen that the spread in $\mathrm{Na}_{7.3}$ and $\mathrm{Fe}_{7.3}$ of EPR lavas relates to the presence and absence of the enriched component that creates T-MORB. It is the presence of this enriched component, therefore, that gives to the EPR data their unique geochemical signature, which contrasts with the slowspreading local vector and the global vector. Although we have documented these local variations using data from the northem EPR, the superfast spreading region of the southem EPR [Sinton et al., 1991] shows very similar chemical characteristics (Figure 29). As Sinton et al. [1991] point out, however, there is substantial variability in $\mathrm{K}_{2} \mathrm{O} / \mathrm{TiO}_{2}$ along the southern EPR.

\subsection{Summary of Observations}

The observations presented above show that once the compositional effects of low-pressure frastionation have been removed, the major element compositions of ocean ridge basalts display certain fundamental systematics. It is our aim to explore the cause of these systematics with quantitative models.

First, on the largest scale of sampling, regional averages of basalt composition from most ridges form a global component 

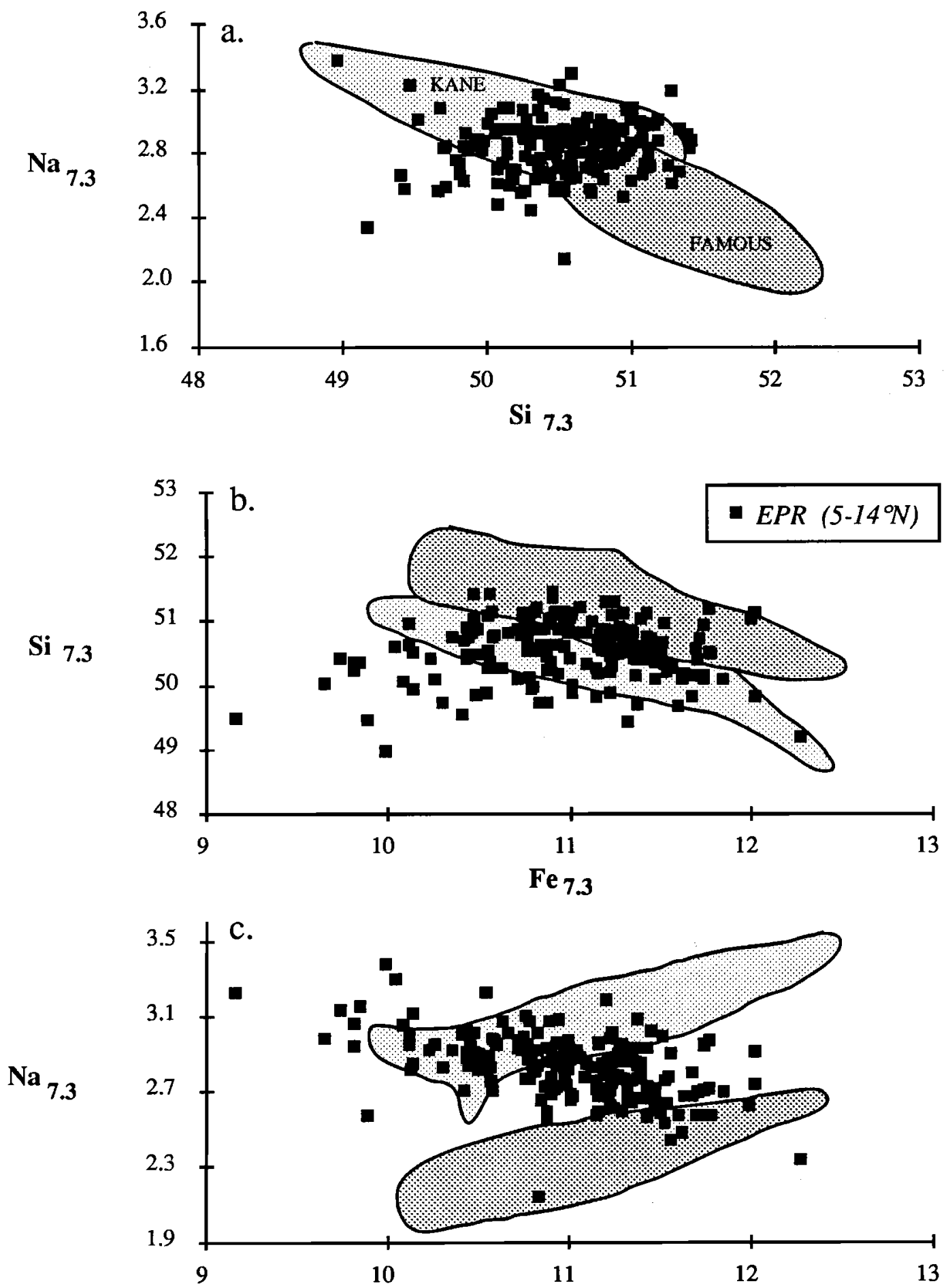

Fe 7.3

Fig. 25. a) $\mathrm{Si}_{7.3}$ vs. $\mathrm{Na}_{7.3}$, b) $\mathrm{Fe}_{73}$ vs. $\mathrm{Si}_{7,3}$, and c) $\mathrm{Fe}_{7.3}$ vs. $\mathrm{Na}_{7.3}$ for individual samples from $5-14^{\circ} \mathrm{N}$ on the EPR (CHEPR data as in Figure 24) compared to Atlantic local i ectors. Fields for the Atlantic enclose individual samples from the MAR near the Kane Fracture Zone and the FAMOUS region, corrected for interlaboratory biases (data sources as in Figure 21; note that data have been normalized to $7.3 \mathrm{wt} \% \mathrm{MgO}$ instead of $8 \mathrm{wt} \% \mathrm{MgO}$ ). EPR local variability is distinct from both the global and the local Atlantic vectors of varialility (see discussion in text). 


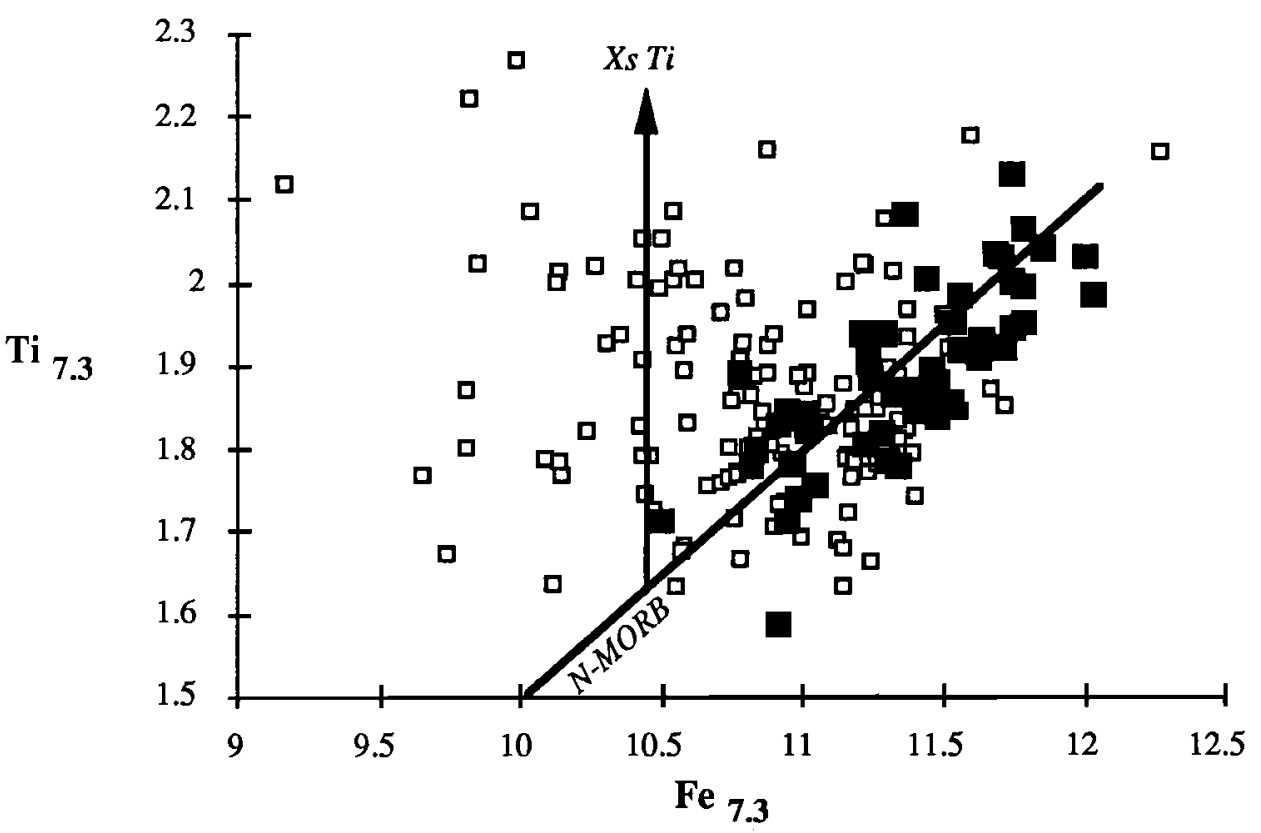

Fig. 26. $\mathrm{Fe}_{7.3}$ vs. $\mathrm{Ti}_{7.3}$ variations for individual sa uples from $5-14^{\circ} \mathrm{N}$ on the EPR (CHEPR data as in Figure 24). The most depleted $\mathrm{CHEPR}$ samples with $\mathrm{K}_{2} \mathrm{O} / \mathrm{TiO}_{2}<0.06 \%$ (solid squares) define a positive trend (N-MORB line), while the entire CHEPR data set is scattered. $\mathrm{Xs} T \mathrm{Ti}$ is defined as $\mathrm{Ti}_{7.3}$ in excess of the $\mathrm{N}-\mathrm{MORB}$ correlation line, as indicated by arrow.

of chemical variability (Figure 15). A key feature of these global chemical systematics is that they also correlate with physical characteristics of the ridge, namely axial depth and crustal thickness (Figures 15 and 18). Some hot spots, however, are anomalous in this global context; regional averages taken over the Galapagos platform, for example, show a relationship between chemistry and depth that is opposite to the global correlations (Figures 15 and 19). Backarc basins exhibit the same correlations es normal ridges, but the data are offset to lower $\mathrm{Fe}_{8.0}$. Iceland generally plots with the global correlations for most elements, but is slightly high in $\mathrm{Na}_{8.0}$ and very high in $\mathrm{Ti}_{8.0}$. Thus, although the global systematics represent one of the most prominent signals in the basalt data, there are exceptions, and no single explanation is likely to account for all characteristics of the data.

When we examine the local variability that is observed among individual samples from individual spreading segments, entirely different chemical systematics emerge. Among samples from slow-spreading ridges, chemical relationships are observed that have the opposite slope to the global $\mathrm{Na}_{8.0}$, $\mathrm{Fe}_{8.0}$ and $\mathrm{Si}_{8.0}$ trends (Figure 21). These characteristics occur over the entire global range of depth and chemistry, and for ridge segments near and far from hot spot influence. On fastspreading ridges, there are two important local chemical components that are distinct from botin the global and the slow-spreading local compositional vectors (Figures 25 and 27). The amount of the enriched component appears to be a dominant control on the range of $\mathrm{Na}_{8.0}$ and $\mathrm{Z}_{8.0}$ observed.

Our challenge, then, is to understand this complicated yet systematic fabric of compositional variability. Our approach is through quantitative models that include complex fractionation, mantle melting and melt segregation processes, and the effects of source heterogeneity. The comparison of these model results with the observations will then allow us to isolate that part of the chemical signal that is governed by the processes of melt formation and migration.

\subsection{Modeling Of Mantle Melting: BACKGROUND AND PREVIOUS WORK}

The basic geochemical observations presented above need to be explained in terms of physical models of melting. differentiation or source heterogeneity. It is clear at the outset that each of the three factors will play some role: no crust can be created without melting; mantle heterogeneity is evident on all scales and in all types of analyses by which the mantle has been studied; complex processes of differentiation are evident in MORB and in all modern models of magma cooling. Our aim is to see how we can distinguish the chemical effects of the various processes in order to better constrain the melting process.

Constraints on the various processes arise from the comparison of MORB compositions to quantitative calculations of the chemical consequences of the possible differentiation and melting processes. The next sections, therefore, consider quantitative modeling approaches. Because we are ultimately concerned with melting processes, modeling of melting is considered in the main body of the text. Complex differentiation processes, however, can also contribute to the chemical systematics observed. Methodology for modeling these complex differentiation processes is presented in 


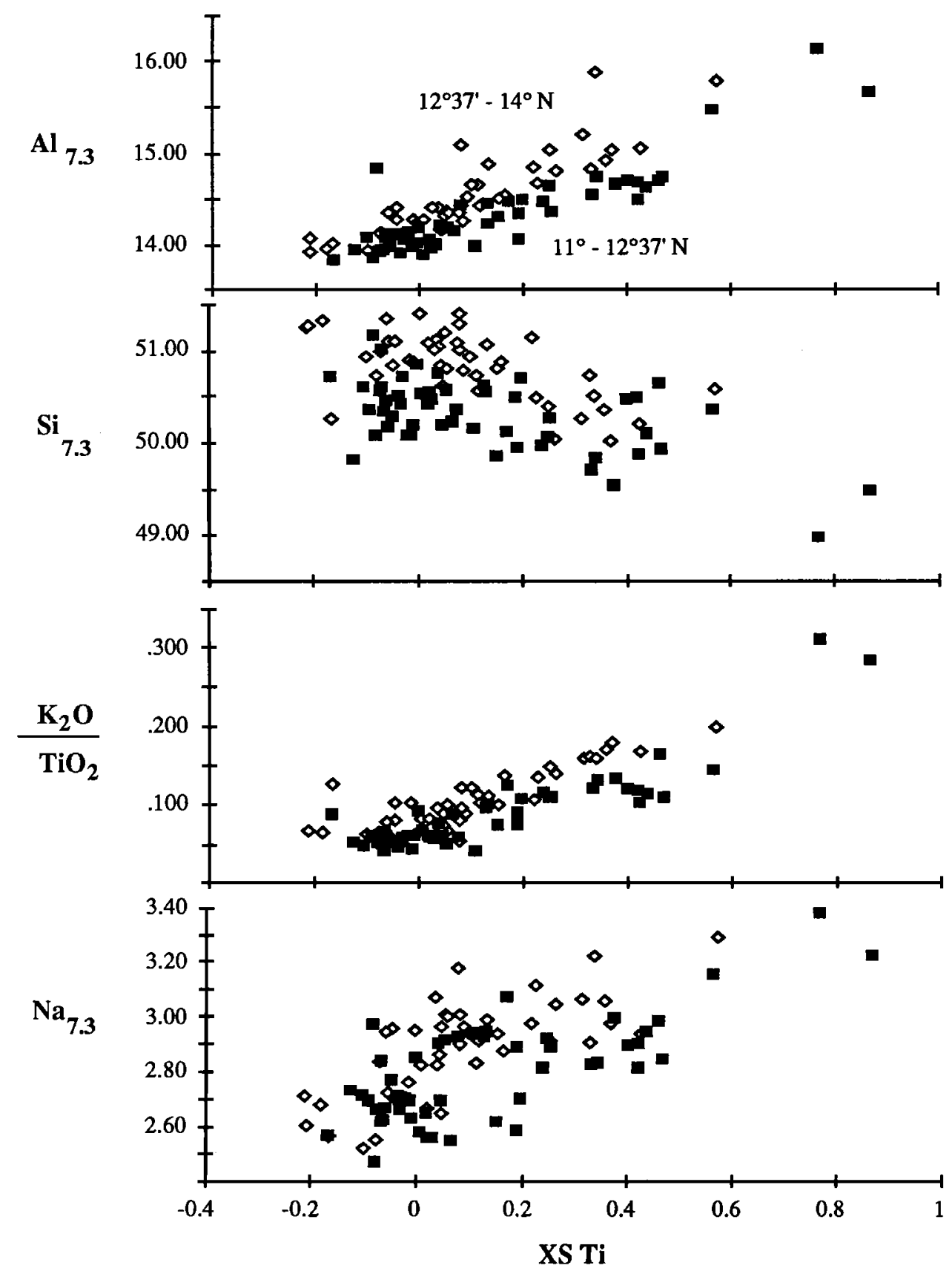

Fig. 27. XS Ti (defined in Figure 26) vs. other chemical parameters for individual analyses from two regions of the northern EPR (CHEPR data as in Figure 24). High XS Ti correlates with $\mathrm{K}_{2} \mathrm{O} / \mathrm{TiO}_{2}$, and therefore reflects the T-MORB component in these samples. Note that samples with high $\mathrm{Na}_{7.3}$ are most influenced by the T-MORB component, therefore it is this component that is controlling much of the EPR local variability.

Appendix $C$, and the results of these calculations are then incorporated into the discussion.

As we saw from the working model, modeling mantle melting requires consideration of three separate but related aspects of the problem. We need quantitative expressions for (a) a melting function, which gives the extent of melting as a function of decompression during adiabatic ascent; (b) a mixing function, which indicates how much of each particular melt composition is produced and pooled; and (c) a chemistry function, which gives the chemical compositions of the melts that are present throughout the melting regime. Each function has its own methodology and uncertainties. Additional 


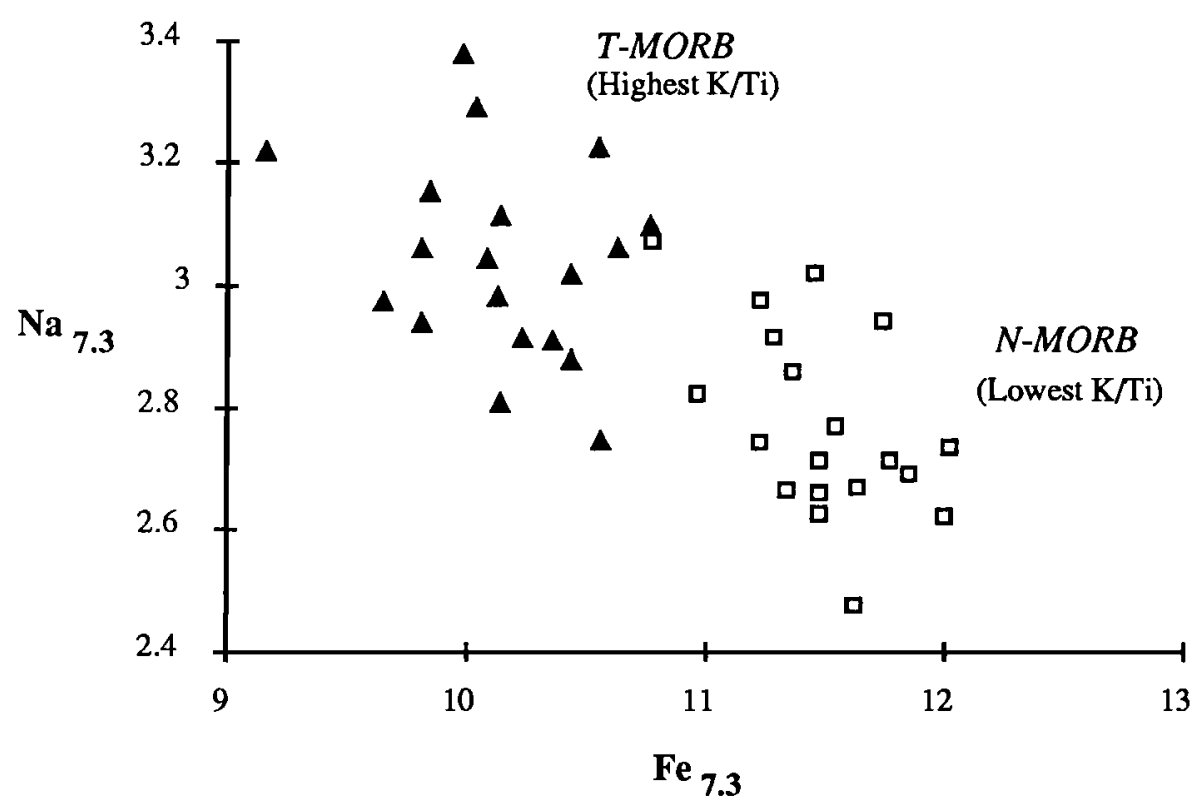

Fig. 28. The control on the $\mathrm{EPR} \mathrm{Na} a_{7.3}-\mathrm{Fe}_{7.3}$ local vector by enriched components. The most enriched samples (filled diamonds; $\mathrm{K}_{2} \mathrm{O} / \mathrm{TiO}_{2}>0.136$ ) have the lowest $\mathrm{Fe}_{7.3}$ and highest $\mathrm{Na}_{7.3}$, while the most depleted samples (open squares; $\mathrm{K}_{2} 0 / \mathrm{TiO}_{2}<0.055$ ) have the highest $\mathrm{Fe}_{7.3}$ and lowest $\mathrm{Na}_{7.3}$. CHEPR data as in Figure 24.

complexities include how much melt is extracted from the solid, how it migrates, and how it chemically reacts on the way to the surface. Quantitative models for mantle melting must consider each of these aspects, for the final chemical composition of the basalt that is delivered to the magma reservoirs, where cooling and fractionation occur, depends on all of them.

\subsection{The Importance of Mechanisms of Melt Extraction}

Before turning to the three functions necessary to calculate melting, it is necessary to consider mechanisms of melt extraction. There are two end-member models that describe the relationship between melting and melt extraction: equilibrium melting, where the melt stays with the matrix as melting proceeds; or fractional melting, where the melt is instanteously separated from the solid as each increment of melt is generated (see equations (5) and (8)).

Whether mantle melting more closely approximates equilibrium or fractional melting will have profound effects on the melting, chemistry and mixing functions. If fractional melting occurs, the melting function will require a greater increase in temperature for a given melt fraction. Perfect fractional fusion could also lead to an effective termination of melting when clinopyroxene disappears from the residue [Presnall, 1969; Hess, this volume]. In all circumstances, fractional fusion leads to less melting and thinner crust for a given pressure of intersection of the solidus.

Fractional melting also influences the chemistry function because successive fractional melts are more depleted in the incompatible major and trace elements, and because the mantle will melt to a lesser overall extent. However, if fractional melts are pooled, the pooled melt composition closely resembles an equilibrium melt produced by melting to the same extent for most elements [Shaw, 1970]. The exception occurs where partition coefficients change significantly as a function of pressure. Then some of the incremental melts may have formed under conditions of very different partition coefficients.

The melting process influences the mixing function primarily through its influence on the physics of upwelling beneath the ridge [e.g., Sleep, 1974; Ahern and Turcotte, 1979; McKenzie, 1984]. Differential flow between melt and matrix depends strongly on the porosity, and therefore on the extraction model. Furthermore, if nelting approaches equilibrium melting, then the greater porosity will lower the effective mantle viscosity, which can lead to the formation of instabilities or focussed flow in the melting regime [e.g., Scott and Stevenson, 1989; Buck and Su, 1989]. As we will see below, this can also affect the mixing function for pooled melts.

Neither pure equilibrium nor pure fractional melting are physically realistic; mantle melting is probably somewhere in between. A more realistic melting model would be that melt segregates once some threshold porosity has been reached. Melt is then continually being removed from the system, but some melt fraction always remains with the residue. This is the "continuous melting" model presented by Langmuir et al. [1977] as one aspect of the more complex "dynamic melting," which conceptually included reaction during ascent and a variety of melt extraction processes. Recent literature has used the term dynamic melting for the original continuous melting model [McKenzie, 1985a, 1985b; Williars and Gill, 1989; 

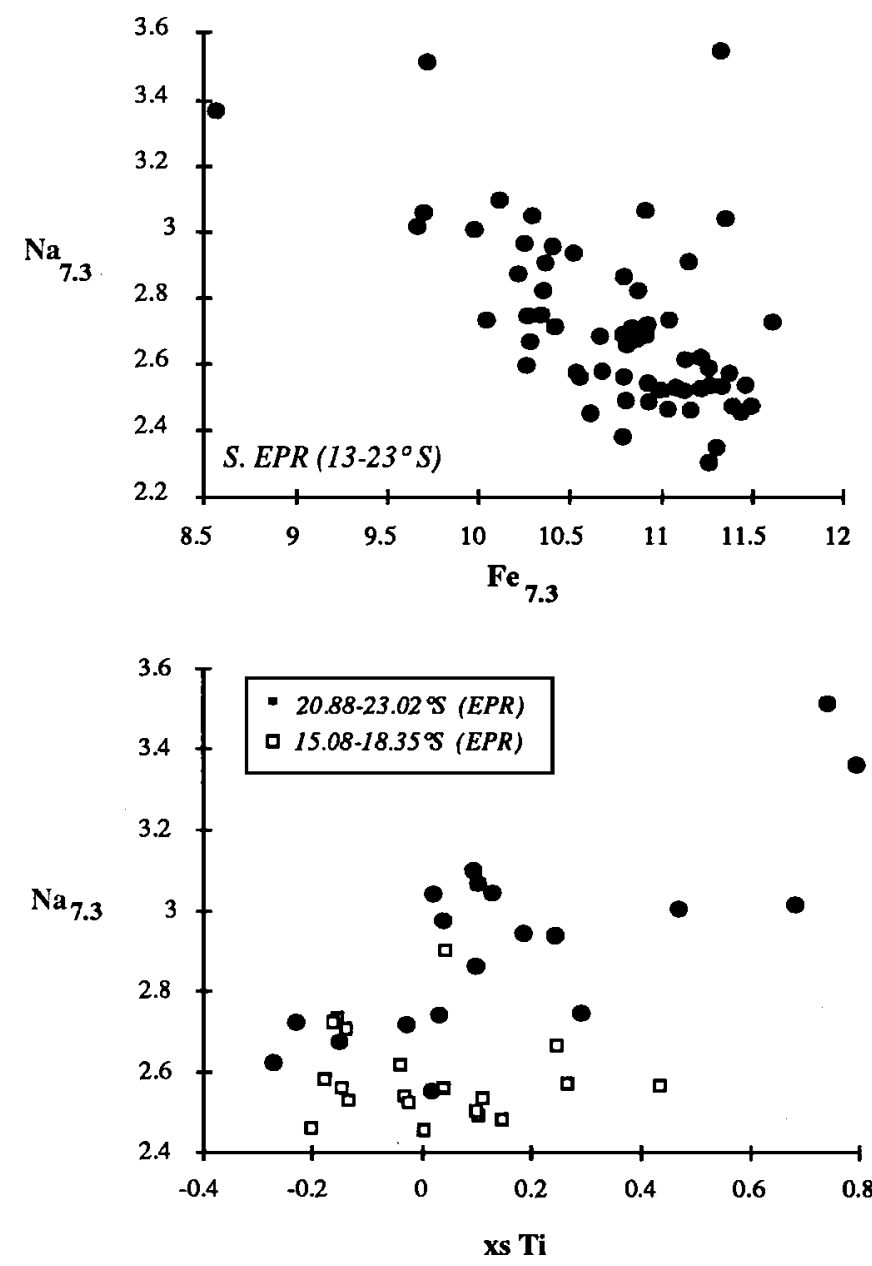

Fig. 29. Basalts from the superfast spreading region of the southern EPR [Sinton et al., 1991] show local systematics similar to those of the northem EPR basalts (compare to Figures 24 and 27).

Elliott et al., 1991]. The important result for geochemical models is that continuous melting leads to effects that are intermediate between equilibrium and fractional melting, depending on the trapped melt fraction.

Even if melting locally approaches fractional melting, reaction of ascending melt with the surrounding solid matrix could lead to chemical effects similar to continuous melting. Measurements of physical properties suggest the possibility of complete extraction of melt, but the fact that melt is generated throughout a large vertical region wot.ld lead to continual replacement by melt transported from below. Further melting would then take place in the presence of a small melt fraction, which is the characteristic of the continuous melting model. The most striking contrast between equilibrium and fractional melting occurs when one solid phase disalpears. At this point, the temperature needed for further fracional melting jumps discontinuously to a much higher value, while equilibrium melting continues progressively with continuously increasing temperature. However, if melt is added to a system that has melted fractionally, melting can continue vithout temperature discontinuities. The net result is that melting can proceed with a small instantaneous porosity. The flow of melt through the system allows a substantial amount of liquid to interact with the solid and produces chemical results similar to continuous melting with a significant porosity. The chemical effects in this case could be interpreted as resulting from a significant residual porosity, even if melt extraction were very efficient and the ultimate porosity in the residual rock was close to zero.

\subsection{The Melting Function}

Figure 1 showed schematically the two important reference slopes for an adiabatically ascending parcel of mantle. Because the solidus has a steeper $\mathrm{dT} / \mathrm{dP}$ slope than the adiabat, mantle that ascends adiabatically will intersect its solidus and begin to melt. The amount of melt generated depends upon the total decompression experienced. The goal of the melting function calculation is to track the "melting path" that a parcel of mantle follows through the melting regime. The energy required to melt is the heat of fusion. Because the ascending mantle is in isolation from its surroundings, the heat of fusion must come from the internal energy of the melting mantle parcel, resulting in a temperature drop. Thus, the "melting path" follows a T-P slope between that of the solidus and the adiabat (Figure 30).

The important parameter in defining the melting path is $(\partial \mathrm{F} / \partial \mathrm{P}) \mathrm{S}$, or the change in melt fraction with change in pressure above the solidus during adiabatic decompression. Estimates of this parameter, which we will call $\gamma$, range from 12\%/kbar [e.g., Ahern and Turcotte, 1979; Cawthorn, 1975; McKenzie, 1984]. Cawthorn [1975] in particular considered how various parameters affect the total amount of melt produced. This parameter $(\gamma)$ provides a crucial tie between the physical and chemical models of melting. For example, if an ascending parcel of mantle intersects its solidus at $20 \mathrm{kbar}$ (about $60 \mathrm{~km}$ depth) and melts $1 \% / \mathrm{kbar}$, then the maximum extent of melting would be $20 \%$. If instead $\gamma$ were $2 \% / \mathrm{kbar}$, then the maximum $\mathrm{F}$ would be $40 \%$, twice as much total melt would be produced, and twice as much crust might be generated. These are substantial differences, and thus it is important to consider what variables contribute to $\gamma$, and how well we know them.

Because much of the information we need to calculate $\gamma$ must come from laboratory experiments where results are given in units of temperature and not energy, it is more convenient to discuss thermal balances. The thermal balance for adiabatic melting is given by four terms: two for the thermal energy available (the slope of the solidus and the slope of the adiabat), and two for the thermal energy required (the heat of fusion, and the $T$ increase above the solidus).

At a given pressure during melting, the thermal energy available for melting comes from the difference in temperature between the solid adiabat and the solidus (Figure 30). (There is an additional term involving the difference between the solid and liquid adiabats, but given the much larger uncertainties in the other parameters, this term can be igr.ored.) 


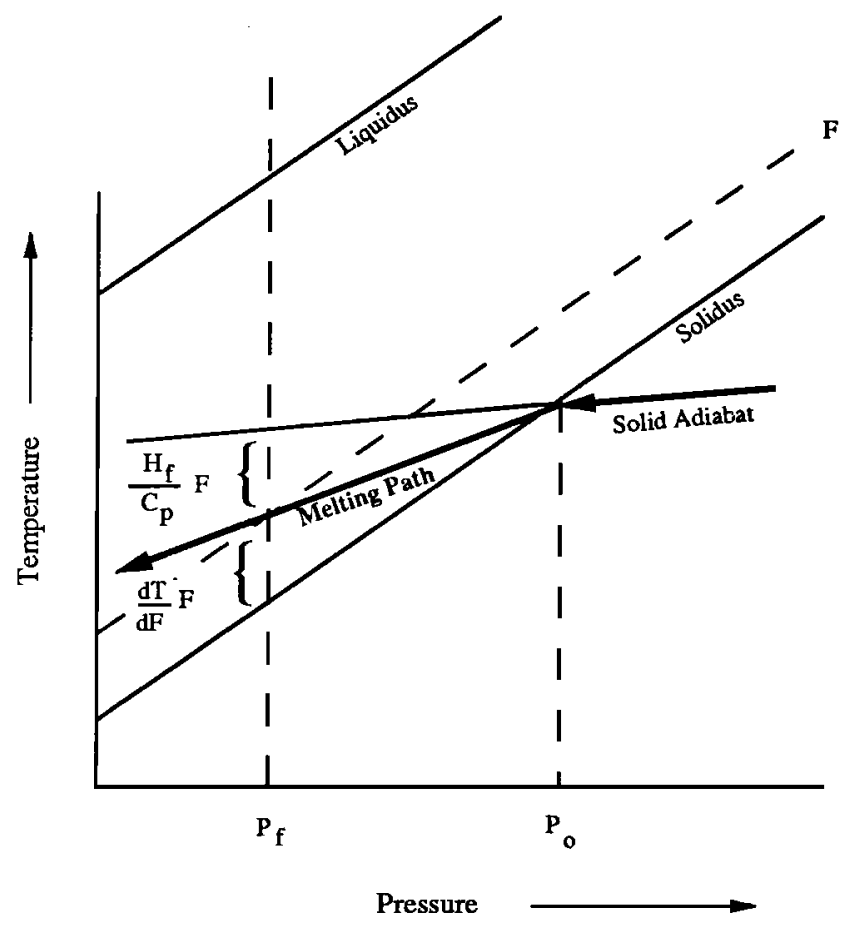

Fig. 30. Schematic pressure-temperature diagram showing the parameters considered in calculating the melting path for an adiabatically ascending mantle parcel, as discussed in section 4.2 in the text. As mantle upwells adiabatically, it intursects the solidus at some pressure $\left(\mathrm{P}_{\mathrm{o}}\right)$ and begins melting; the melting path has a steeper slope than the solid adiabat because there is a temperature drop associated with the heat of fusion $\left(\mathrm{H}_{f}\right)$. At any given pressure $\left(\mathrm{P}_{\mathrm{f}}\right)$ less than $P_{0}$, the energy available for melting can be expressed as the temperature difference between the solidus and the extension of the solid adiabat. This temperature difference is partitioned between the temperature drop associated with the heat of fusion, and the temperature increase above the solidus required to melt to an extent $F$. $C_{p}$ is the heat capacity, and $\mathrm{dT} / \mathrm{dF}$ is the spacing of the contours of $\mathrm{F}$ on this diagram.

For a one-component system, the solid phase melts at a single temperature at any one pressure. For a multi-component system like the mantle, however, melting occurs over a large temperature interval, from $0 \%$ melting at the solidus to $100 \%$ at the liquidus. Thus, at any given pressure, it takes a given temperature change above the solidus to melt a given amount, and this value for $\mathrm{dT} / \mathrm{dF}$ determines the melting contours on a $\mathrm{P}$ $\mathrm{T}$ diagram (e.g., Figure 1). The simplest way to contour $\mathrm{F}$ as a function of $P$ and $T$ is to assume a linear spacing of melting contours from solidus to liquidus, or a constant value of $(\mathrm{dT} / \mathrm{dF})$. The temperature increase required to melt a specific amount $(F)$ would thus be $F^{*}(d T / d F)$.

The heat of fusion $\left(\mathrm{H}_{\mathrm{f}}\right)$ is the amount of energy required to melt $100 \%$. This heat is obtained by cooling the mantle, and the energy yielded by each degree of cooling is given by the heat capacity, $C_{p}$. If we assume that the heat of fusion is evenly distributed between liquidus and solidus, and that $\mathbf{H}_{f}$ and $C_{p}$ remain constant over the melting interval, then the temperature drop upon melting is $F^{*}\left(H_{f} / C_{p}\right)$.
For a given decrease in pressure from $P_{o}$ (the pressure of intersection of the solidus) to $P_{f}$ (the final pressure), the thermal balance between these four terms may be written as:

$$
\left(P_{\mathrm{f}}-\mathrm{P}_{\mathrm{o}}\right) *\left[(d T / d P)_{a d b}-(d T / d P)_{s o l}\right]=F^{*}\left(H_{f} / C_{p}\right)+F^{*}(d T / d F)
$$

For incremental changes in pressure, the extent of melting as a function of pressure is:

$$
\gamma=\left[(\mathrm{dT} / \mathrm{dP})_{\text {adb }}-(\mathrm{dT} / \mathrm{dP})_{\text {sol }}\right] /\left[\mathrm{H}_{\mathrm{f}} / \mathrm{C}_{\mathrm{p}}+\mathrm{dT} / \mathrm{dF}\right]
$$

Thus we can calculate the amount of melting produced upon decompression by substituting into equation (18) values for the slope of the solidus, the slope of the adiabat, the heats of fusion and heat capacities of mantle materials, and a function relating temperature above the solidus to extent of melting. The uncertainty in $(\partial \mathrm{F} / \partial \mathrm{P})$ thus relates to the uncertainties in these various parameters. Below we consider typical values for these parameters, as well as the uncertainties and complexities that might realistically be involved.

A typical value for $(\mathrm{dT} / \mathrm{dP})_{a d b}$ is $1^{\circ} \mathrm{C} / \mathrm{kbar}$, though it may be as high as $2 \%$ kbar [e.g., Cawthorn, 1975]. These are small numbers compared to the slope of the solidus (typically $12 \% / \mathrm{kbar}$; see below), and so variations in this number do not lead to large variations in $(\partial \mathrm{F} / \partial \mathrm{P})$.

In principle, the solidus should change slope as the mantle assemblage changes from plagioclase- to spinel- to garnetlherzolite with increasing pressure [e.g., Presnall et al., 1979]. These differences, however, are not obvious in the existing experimental data on natural systems. McKenzie and Bickle [1988] made an extensive compilation of data on the mantle solidus. Their compilation is reproduced in Figure $31 a$ for the pressure interval from 0 to $40 \mathrm{kbar}$, which is relevant to magma genesis in the modern earth. The open symbols are those experiments where solid and liquid were present, while the closed symbols are those where there was no liquid; in principle, the mantle solidus should be the single line that separates the two symbols. It is clear from the figure, however, that there is significant overlap in the data. Much of this variability may reflect real differences among starting compositions and experimental techniques, rather than statistical error. The solid lines in the figure are drawn such that one is below most of the open symbols, while the other is above most of the closed symbols. These two lines illustrate the uncertainty in our knowledge of the solidus (about $60^{\circ} \mathrm{C}$ ). The zero pressure intercept of the solidus could be anywhere from $1095^{\circ} \mathrm{C}$ to $1155^{\circ} \mathrm{C}$. The slope is constrained to be between 11.5 and $13 \% / \mathrm{kbar}$. Thus there are considerable uncertainties in both the slope and intercept of the solidus. A regression of all the data in Figure $31 a$ leads to the equation: [ $T$ $=1139+11.4 * \mathrm{P}]$. If one considers only the experiments that bracket the solidus to better than $50^{\circ} \mathrm{C}$, the regression yields: $[\mathrm{T}=1118+12.7 * \mathrm{P}]$. In our calculations we consider the effects of variations in both the intercept and slope of the solidus.

Common values for the heats of fusion and heat capacities of mantle minerals are taken to be $100 \mathrm{cal} / \mathrm{gm}$ and $0.3 \mathrm{cal} / \mathrm{gm} /{ }^{\circ}$, respectively [e.g., Carmichael et al., 1974]. However, the heat of fusion and heat capacity may vary significantly as a function 

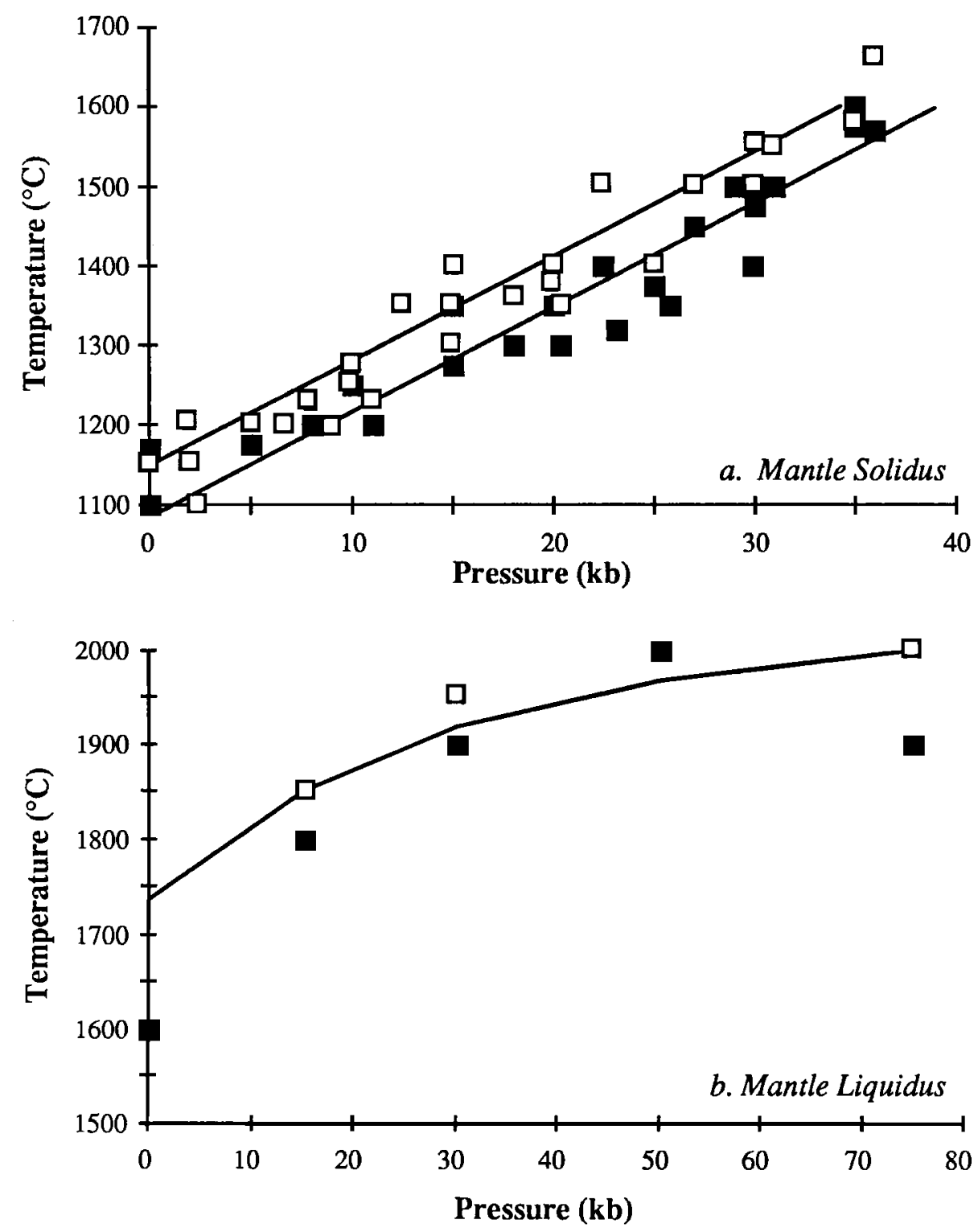

Fig. 31. a) Pressure-temperature diagram for estimating the slope and intercept for the mantle solidus. Data for both panels as compiled by McKenzie and Bickle [1988]. Filled squares show results from experiments in which only solid was present; open squares show results of experiments in which both solid and melt were present; lines are drawn so that the lower line is below most of the solid+melt data, and the upper line is drawn so that it is above most of the solid-only data. The offset in the lines suggest that the uncertainty in estimating the solidus is about $60^{\circ} \mathrm{C}$ at a given pressure. $b$ ) Pressure-temperature diagram for estimating mantle liquidus. Filled squares show experiments in which both solid and melt are present, and the open squares show experiments in which only melt is present. The curve shows the estimate of the liquidus of McKenzie and Bickle [1988].

of temperature, mineralogy and melt fraction [Cawthorn, 1975]. Hess [this volume] evaluates the most recent experimental data, and suggests an average value for the heat of fusion for mantle melting of $180 \mathrm{cal} / \mathrm{gm}$. Varying the heat of fusion from 100 to $180 \mathrm{cal} / \mathrm{gm} /{ }^{\circ}$ has a large effect on $\gamma$, thus we consider the effects of both values in our calculations.

The way in which $F$ is contoured in the melting interval must come directly from melting experiments. Existing data from peridotite melting experiments suggest that two important features must be taken into account. First, the melting interval narrows with pressure as the liquidus and solidus approach each other; and second, melting contours are closer together near the solidus.

The narrowing of the melting interval with pressure requires knowledge of both the mantle solidus and liquidus as a function of pressure. There are sparse data for the mantle liquidus 
because the high temperatures involved are difficult to achieve in the laboratory. The available data [Takahashi, 1986] are presented in Figure 31b. McKenzie and Bickle [1988] calculate an uncertainty in the liquidus of $7{ }^{\circ} \mathrm{C}$, but in practice the uncertainties in the liquidus are much greater than this value. For example, the minimum temperature interval over which the liquidus is bracketed is $50^{\circ} \mathrm{C}$. Moreover, there are few data at low pressures, and almost any curve can be drawn for the liquidus below $15 \mathrm{kbar}$. Despite the lack of constraints on the slope of the liquidus, we can make a rough approximation of the narrowing of the melting interval with pressure. For the purposes of the melting calculations in this paper, we assume that the melting interval decreases by a factor of two in roughly $45 \mathrm{kbar}$ (from experiments of Takahashi, 1986), and that the spacing between the melting contours decreases proportionally.

Some experiments suggest that substantial melt is generated over a small temperature interval near the solidus [see Figure 2 in Jaques and Green, 1980]. Knowledge of the melting relationships near the solidus is quite limited because there are few experimental data between 0 and $15 \%$ melting. During progressive melting of peridotite, it appears that clinopyroxene disappears as a residual phase after about $20 \%$ melting [e.g., Jaques and Green, 1980; Hess, this volume]. The subcalcic clinopyroxenes that exist at higher pressure might last to considerably higher melt fractions, however. In the absence of better experimental constraints, we assume that a change in melting behavior results from the disappearance of clinopyroxene at $22 \%$ melting, and that melting contours are more closely spaced for less than $22 \%$ melting, and more widely spaced for greater than $22 \%$ melting. We use $3.5^{\circ} \mathrm{C} / \%$ for $\mathrm{F}<22 \%$ melting, and $6.8^{\circ} \mathrm{C} / \%$ for $\mathrm{F}>22 \%$. These contour intervals are appropriate for melting at $1 \mathrm{~atm}$, and become compressed proportionally with the melting interval with increasing pressure. Figure 32 shows the melting contours resulting from these relationships. Note that we do permit melting to occur beyond $22 \%$ melting, where clinopyroxene may disappear, because residual porosity or melt fluxing from below will permit additional melting to take place, as discussed above in section 4.1 .

Substituting into equation (18) the values of $1 \% \mathrm{kbar}$ for the slope of the adiabat, $13^{\circ} / \mathrm{kbar}$ for the slope of the solidus, 0.3 $\mathrm{cal} / \mathrm{gm} /{ }^{\circ}$ for the heat capacity, $3.5 \%$ and $6.8 \%$ for $\mathrm{dT} / \mathrm{dF}$ (at less than and greater than $22 \%$ melting, respectively), and (1$\mathrm{P} / 88$ ) for the pressure correction on the melting contours, we arrive at:

$$
\gamma=-12 /\left[\left(\mathrm{H}_{\mathrm{f}} / 0.3\right)+350(1-\mathrm{P} / 88)\right] \quad \text { for } \mathrm{F}<0.22
$$

and:

$$
\gamma=-12 /\left[\left(\mathrm{H}_{\mathrm{f}} / 0.3\right)+680(1-\mathrm{P} / 88)\right] \quad \text { for } \mathrm{F}>0.22
$$

Figure 32 shows adiabatic melting paths for mantle that intersects the solidus at $30 \mathrm{kbar}$, calculated from equations (19) and $(20)$ for two different values of the heat of fusion (100 and $180 \mathrm{cal} / \mathrm{gm})$. Note that the total amount of melting differs considerably for the two cases. The estimates of $\gamma$ given by equations (19) and (20) range from values similar to that used by $K L 87(1.2 \% / \mathrm{kbar})$ to around $2 \% / \mathrm{kbar}$ for the lower estimate

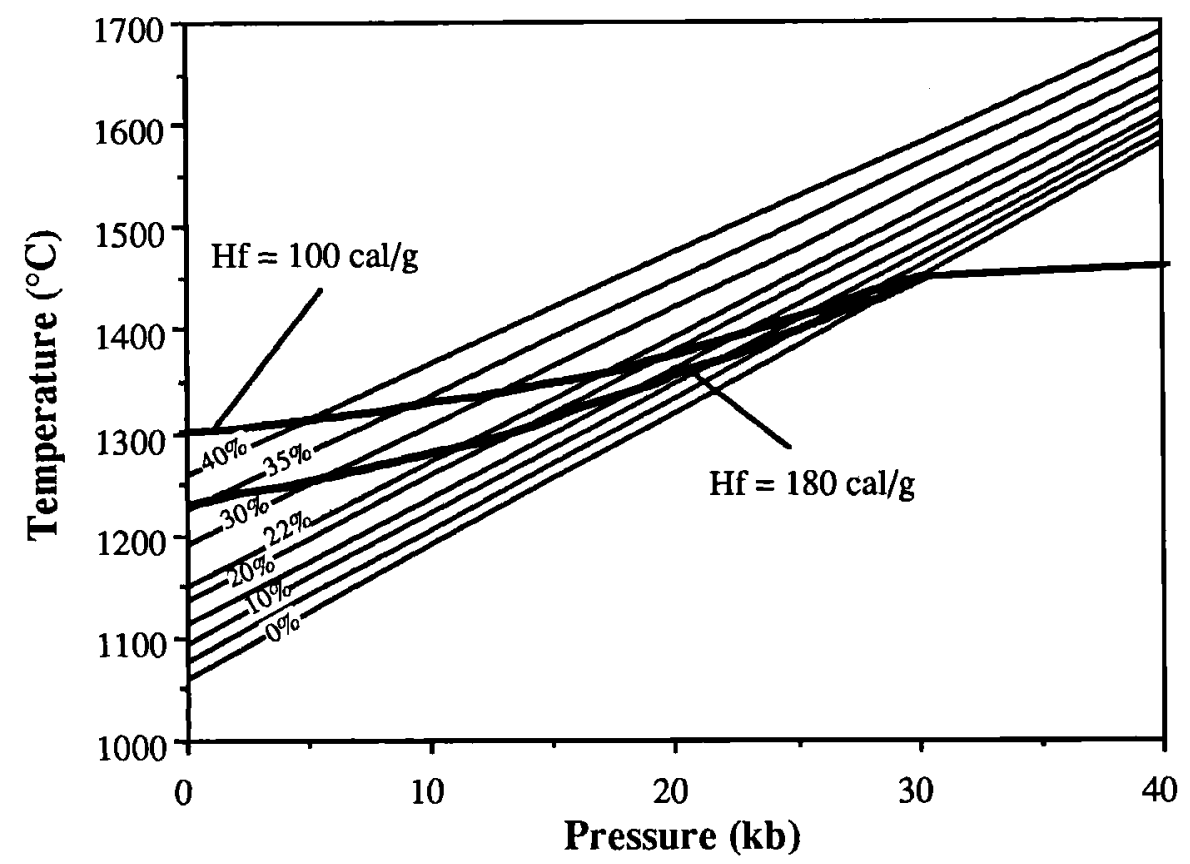

Fig. 32. Pressure-temperature diagram contoured for the extent of melting (\%F) showing melting paths for mantle that intersects the solidus at $30 \mathrm{kbar}$. Melting paths and melting contours calculated from equations given in section 4.2 in the text. The two paths are for two different values for the heat of fusion $\left(\mathrm{H}_{\mathrm{f}}\right)$, as indicated. 
of the heat of fusion at high pressure. For comparison, the melting function used by McKenzie and Bickle [1988] averages $1 \% / \mathrm{kbar}$ for $\mathrm{P}_{\mathrm{o}}=35 \mathrm{kbar}$, and $1.6 \% / \mathrm{kbar}$ for $\mathrm{P}_{\mathrm{o}}=15 \mathrm{kbar}$.

The melting paths in Figure 32 are curved because the instantaneous slope depends on both the extent and pressure of melting. During the initial stages of adiabatic melting, the pressure is high, and the extent of melting low. Both of these factors lead to compressed melting contours and larger amounts of melting per unit of pressure release. The melting path during the initial stages of melting has a steeper $\mathrm{dT} / \mathrm{dP}$ slope because more energy (temperature) is required for each unit of pressure drop.

In order to calculate the total amount of melting along a given trajectory, equations (19) and (20) can be integrated:

$\mathrm{F}_{\max }(<22 \%)=3.015 \ln \left[\left(\mathrm{H}_{\mathrm{f}} / 0.3+350-3.98 \mathrm{P}_{\mathrm{f}}\right) /\left(\mathrm{H}_{\mathrm{f}} / 0.3+350-\right.\right.$ $\left.\left.3.98 \mathrm{P}_{\mathrm{o}}\right)\right]$

and for the portion above $22 \%$ melting:

$\mathrm{F}_{\max }(>22)=1.546 \ln \left[\left(\mathrm{H}_{\mathrm{f}} / 0.3+683-7.76 \mathrm{P}_{\mathrm{f}}\right) /\left(\mathrm{H}_{\mathrm{f}} / 0.3+683-\right.\right.$ $\left.\left.7.76 \mathrm{P}_{22}\right)\right]$

where $P_{22}$ is the pressure of the mantle parcel at $22 \%$ melting and equals (1.0757 $\left.P_{0}-18.1\right)$.

A melting path for fractional melting is less well constrained. The principal uncertainty is that the temperature of the mantle solidus continually rises as melt is removed, and this temperature increase cannot be determined from the equilibrium melting conditions of the experimental data. To approximate the effects of fractional melting, we use a dT/dF value of $6 \%$ melting for $\mathrm{F}<22 \%$, and $8 \%$ melting for $\mathrm{F}>22 \%$. Using these values, of course, leads to correspondingly less melting per unit of pressure release. For evaluation of mantle heterogeneity, we assume that the mantle solidus increases by $20^{\circ}$ for each unit increase in the $\mathrm{Mg} \#[\mathrm{Mg} /(\mathrm{Mg}+\mathrm{Fe})$, atomic ratio]. Both of these values need to $b e$ refined by further experimental work.

\subsection{The Mixing Function and the Shape of the Melting Regime}

The amount of melt generated under the ridge depends on the upwelling pattern as well as the ambient temperature of the underlying mantle. In addition, melts generated by fractional or equilibrium melting come from different depths in the melting regime, and hence there are chemical corsequences associated with the melting process. In this section, we show how melts can be summed for different melting regimes and melting processes, and how the summing is relatively insensitive to the many variations in the "shape" of the melting regime beneath a ridge. A more thorough consideration of the effects of the shape of the melting regime is presented by Plank and Langmuir [1992].

\subsubsection{An Illustrative Incremental Model}

In general, because melting occurs wherever mantle upwells above its solidus, the physical form or "shape" of the melting regime is defined by the upwelling pattern. Consider a simple, two-dimensional, comer-flow pattem that results from passive upwelling in response to spreading [e.g., Ahern and Turcotte, 1979; Phipps Morgan, 1987; McKenzie and Bickle, 1988; Figure $33 a$ ]. The base of the melting regime is the mantle solidus, and the boundaries of the melting regime are defined by where mantle flows horizontally and no longer melts. Given a solidus, a melting function, and mantle temperature on the solidus, melting can be contoured in the nelting regime. The end-points of the mantle flow paths (1.4) from the melting regime in Figure $33 a$ are highlighted on the P-T diagram in Figure $33 b$.

Figure $34 a$ illustrates schematically what happens in the simplified melting regime of Figure 33 during an increment of spreading. Boxes are used to represent units of mantle passing through the melting regime, with one box length equal to one increment of spreading. The shading reflects the amount of melt that is produced upon upwelling. Open boxes indicate no melting. During an increment of time, the plates spread, and all the white boxes as well as the boxes at the boundaries of the melting regime move one horizontal step. All the other boxes move up to replace the void and melt an additional increment. The total number of increments that each box has melted is proportional to the distance above the solidus.

Consider first an equilibrium melting model, where the melt remains in contact with the mantle all the way to the top of each column, and then is extracted. The melt fraction within each box increases upon decompression, and no melt leaves the matrix until the box reaches the top of its column. The melts are then tapped from the boxes at the top of each column in the melting regime, and these melts are pooled to form the oceanic crust. KL87 and McKenzie and Bickle [1988, hereafter MB88] presented pooled melt compositions produced by this kind of melting and mixing model, although neither study described the melting regime in this way.

In order to calculate the composition and volume of the melt mixture that forms the oceanic crust, it is useful to consider the steady-state residue of mantle melting that results from melt extraction within a given melting regime. At steady-state, the residue of mantle melting is the column of mantle that emerges outside the melting regime - the residual mantle column (RMC) (Figure 34a). The RMC is the net result of the integrated melting and extraction processes that occur within the melting regime. Each increment of spreading creates a new section of oceanic crust and a new RMC. Each box in the RMC, back-tracked along its flow path, represents a column in the melting regime. For example, the uppermost box in the RMC in Figure $34 a$ originated from six increnents of melting in the central column of the melting regime. Thus, the twodimensional melting regime is reduced to a one-dimensional residual column.

Because the RMC is the complement to the steady-state pooled melt that forms the ocean crust, the RMC may be used to calculate important properties of the ocean crust, such as its composition and thickness, and the mean extents and pressures of melting by which it was derived. To calculate these properties, it is only necessary to consider what has happened to produce each box in the RMC. The final mean extent of 
a.

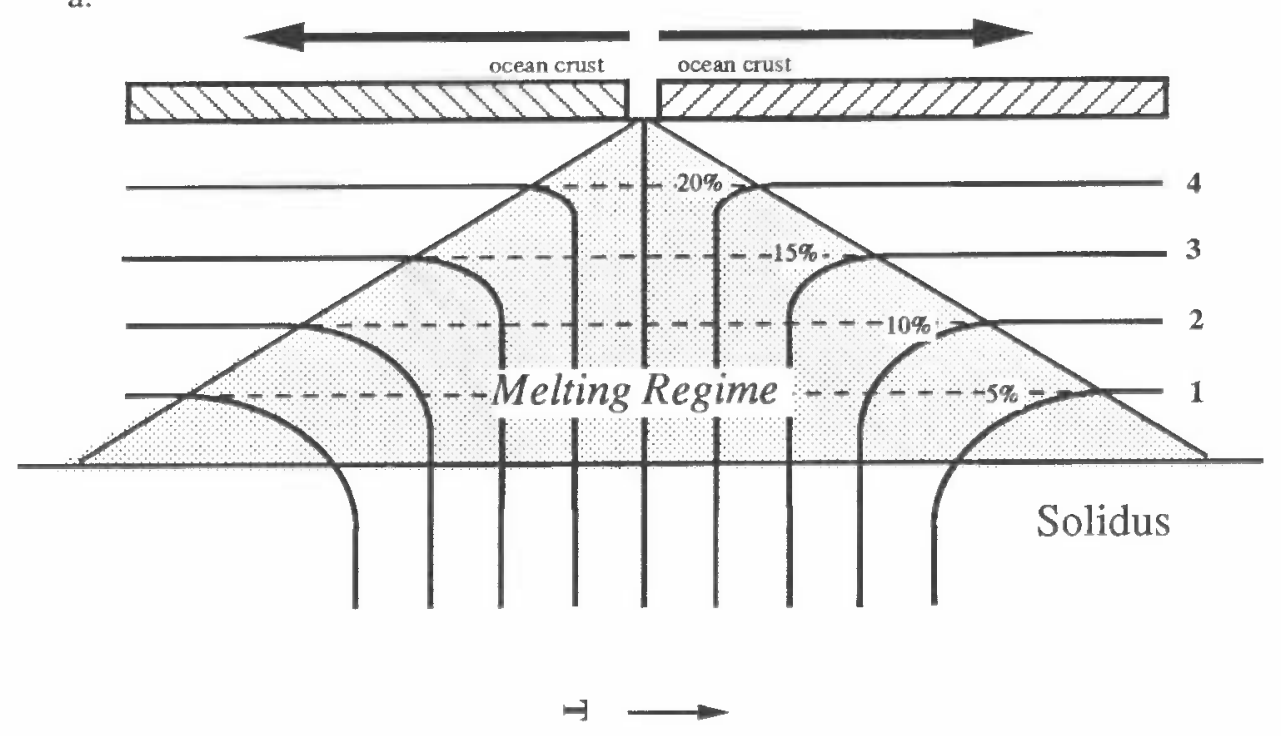

b.

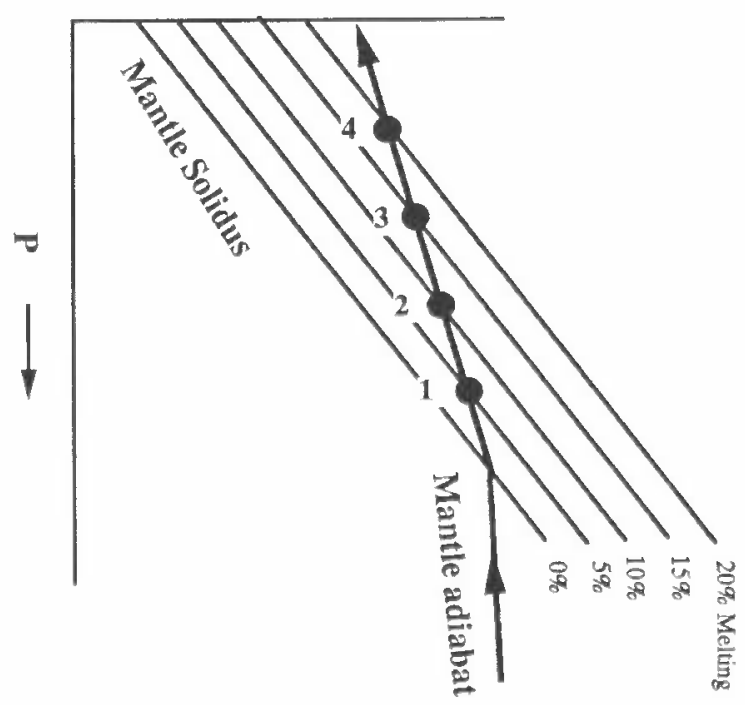

Fig. 33. a) Schematic view of the melting regime for simple comer flow circulation beneath the ridge resulting from passive upwelling as a result of spreading. Solid lines (without arrows) are mantle flow lines; the shaded region is the palting adiabatic melting resulting from upwelling and intersection of the mantle solidus. The region above the solidus is contoured for the extent of melting. The numbered dots (1-4) correspond to the maximum extent of melting that each numbered flow line in Panel (a) undergoes. Thus, the single melting path in (b) is a representation of the melting systematics that occur in the two-dimensional meiting regime shown in Panel (a).

melting $(\overline{\mathrm{F}})$, mean pressure of melting $(\overline{\mathrm{P}})$, and composition of the pooled melt can be calculated simply by averaging the melt associated with each of the boxes in the RMC. The bookkeeping for this illustrative calculation is shown in Figure $34 a$, where the height of each box equals $1 \%$ melting and 1 unit pressure.

This simple incremental model can be used to illustrate how the mean properties of the oceanic crust can be calculated from the RMC.
1. The mean extent of melting, $\bar{F}$ is the total amount of melt produced, divided by the total amount of mantle that has melted during each increment of spreading. Since the RMC is the amount of mantle processed through the melting regime during each increment of spreading, $\bar{F}$ is the total melt divided by the total area of the RMC. $\overline{\mathbf{F}}$ is calculated in Figure $34 a$ by summing the melt associated with all the boxes in the RMC, and dividing by the total number of boxes in the RMC. Although the maximum amount of melting $\left(F_{\max }\right)$ for the 


\section{a. Equilibrium}

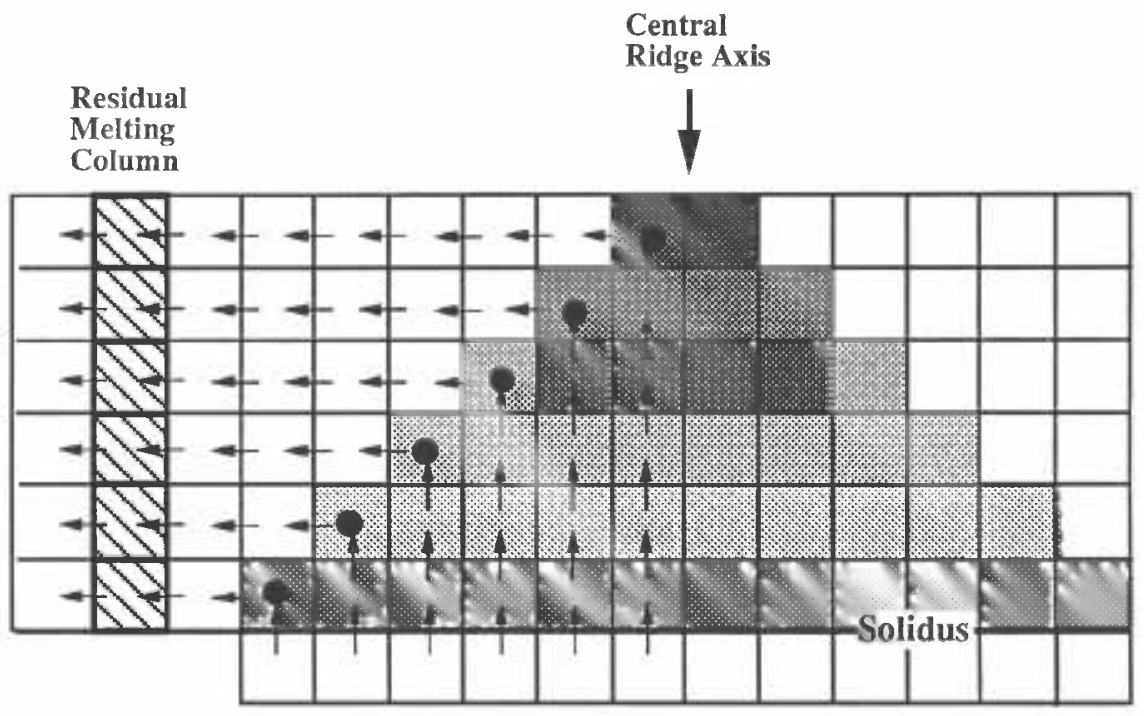

P $\quad$ RMC (E.P. Cl/Col

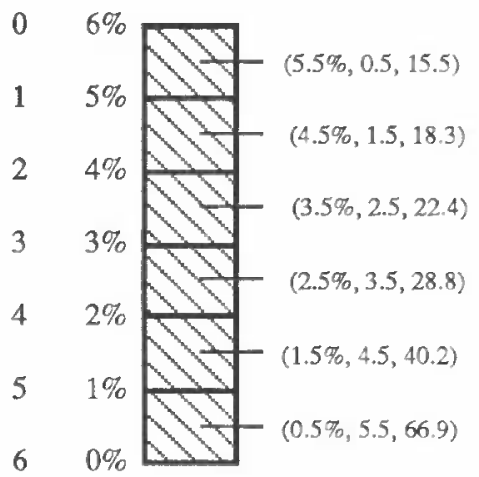

$\overline{\mathrm{F}}=3 \%$
$\mathrm{~h}_{\mathrm{C}}=0.18 *$ box height
$\overline{\mathrm{C}}_{\mathrm{L}}=23 * \mathrm{C}_{\mathrm{o}}$
$\overline{\mathrm{P}}=2$

b. Fractional

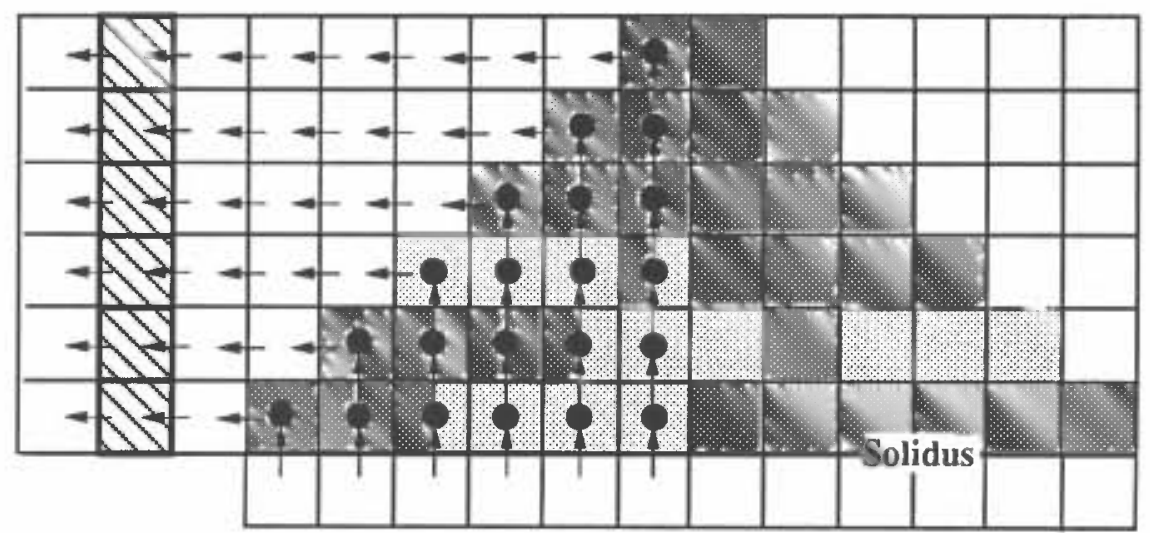

P $\quad$ F $\quad \mathrm{RMC} \quad$ (F,P. $\mathrm{Cl} / \mathrm{Co})$

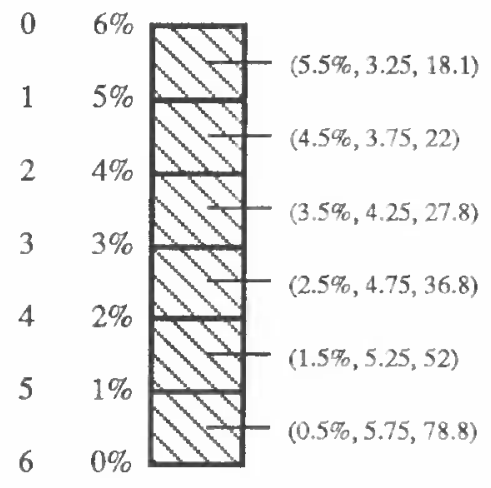

$\overline{\mathrm{F}}=3 \%$
$\mathrm{~h}_{\mathrm{c}}=0.18 *$ box height
$\overline{\mathrm{C}}_{\mathrm{L}}=28 * \mathrm{C}_{\mathrm{o}}$
$\overline{\mathrm{P}}=4$


melting regime in Figure $34 a$ is $6 \%, \overline{\mathrm{F}}$ is half the maximum, or 3\%. [Note: In our figures, the mantle has not been compacted to account for the volume lost by melt extraction. Thus, the length of the RMC is the total pressure interval of melting, and not the true residual mantle column compacted for melt loss. This introduces small errors in the calculations of crustal thickness, but allows simpler illustrations of the relationship between the RMC, the melting regime, and crustal thickness.]

2. Because the melts that make up the ocean crust have been derived from various depths in the melting regime, the ocean crust reflects a mean of the pressures of equilibration of the individual melts. If the melts undergo no re-equilibration once separated from the solid residue of meling, then the mean pressure $(\bar{P})$ can be calculated as a weighted mean of the individual melts. In Figure $34 a, \overline{\mathrm{P}}$ is 2 pressure units, which is less than half the pressure interval because more melt is generated in the shallow levels of the melting regime. [Note: $K L 87$ defined a "mean pressure" as the pressure at which half the total melt formed shallower and half formed deeper. This is actually a median pressure, and differs slightly from the mean pressure defined here, which is a true mean. A true mean $\overline{\mathrm{P}}$ is conceptually and computationally easier to compare to mean compositions.]

3. If all the melt pooled from the melting regime forms the ocean crust, then the total melt produced during each increment of spreading would equal the thickness of the ocean crust, $h_{c}$, once differences in densities (about 10\%) are accounted for. Because the RMC is the total amount of mantle processed through the melting regime during each increment of spreading, if all the melt is extracted, the crustal thickness equals the height of the RMC multiplied by the mean extent of melting. If the boxes in Figure $34 a$ were each $10 \mathrm{~km}$ in height, then $(0.03 * 60) / 0.9$ or $2 \mathrm{~km}$ of crust would be generated during each unit of spreading.

4. For equilibrium melting, the liquid composition can be calculated anywhere in the melting regime given the source composition, the bulk distribution coefficient that applies to the appropriate extent of melting, and the batch melting equation (equation 5). The composition of the total aggregate melt from the entire melting regime can then be calculated by weighting the melt composition by the amount of melt with that composition (a weighted mean). For the example in Figure $34 a$, an element with $\mathrm{D}=0.01$ would be enriched about 23 -fold in the final aggregate melt over the source concentration. Note that this is very similar to a batch melt calculated for the same $\overline{\mathrm{F}}$ (using equation 5 , a $3 \%$ batch melt $=25$-fold enrichment for $D$ $=0.01$ ).

This latter point, that the composition of the pooled melt from the melting regime closely resembles a simple batch melt calculated from $\bar{F}$, is particularly important. It means that a MORB may be inverted to calculate an accurate $\bar{F}$ by assuming simple batch melting, even if the sample was derived by mixing of melts from throughout the melting regime.

\subsubsection{Effects of Fractional Melting}

Consider now the incremental model for fractional melting, where melt is extracted from the solid mantle as soon as it forms. This process is illustrated schematically in Figure $34 b$, where the solid circles represent points at which melt is extracted. During an increment of spreading, each box that upwells will melt one increment. These increments are then extracted from each box and pooled at the ridge axis. Note that in this model, melt is extracted from all depths, not just at the top of each of the melting columns, as for the equilibrium model presented above. Pooled melts derived by fractional melting thus reflect higher mean pressures. For the fractional melting example shown in Figure $34 b$, the mean pressure of melt extracted from the central melting column is half the total pressure interval ( $3.25 \mathrm{kbar}$ ), because equal increments of melt are extracted at each depth. This mean pressure contrasts with that for the equilbrium model, which is the shallowest pressure for the central column $(0.5 \mathrm{kbar})$. The mean pressure for the fractional melts pooled from the entire melting regime in Figure $34 b$ is twice that for the equilibrium case.

Although the most dramatic difference between pooled fractional and equilibrium melts is the mean pressure, there are other differences. The composition of the pooled melt will be slightly different for fractional vs. equilibrium melting. For the fractional case, the pooled melt derived from each vertical column has an equivalent composition to an accumulated fractional melt (AFM) melted to the maximum extent (e.g., 6\% for the central column). Thus, the AFM equation of Shaw [1970] was used to calculate the concentrations in the melt associated with each column in the melting regime, or box in the RMC, in Figure $34 b$.

$$
C_{L}=\left(C_{0} / F\right)^{*}\left[1-(1-F)^{(1 / D)}\right]
$$

Fig. 34. A schematic incremental view of melting beneath the ridge and a calculation of the mean properties of the pooled melt from the melting regime. Arrows indicate solid mantle flow. The shape of the melting regime (shaded) is defined by the upward component of mantle flow and therefore by the boxes that move upward; the point where mantle flow is horizontal defines the upper boundary of the melting regime. Panel (a) is for equilibrium melting and Panel (b) for fractional melting. The shading increases in intensity with increasing amount of melt in each box as it moves upward; melt is pooled from only the top boxes of each of the columns (boxes with large dots at left side of melting regime). Also shown is the residual melting column (RMC) which is the steady-state residue of melt extraction. The extent of melting (F) and pressure of melting $(P)$ in the melting regime are in arbitrary units. Values presented at the right of the diagram show calculations that result from a consideration of the melting systematics represented by the RMC. The numbers in the parentheses are the $\bar{F}, \bar{P}$ and mean concentration of an element with $D=0.01$ (mean $C_{1} / C_{0}$ ) of the melt associated with each box in the RMC or column in the melting regime. $\bar{F}, \bar{P}, C_{L}$ (mean $C_{L} / C_{o}$ ) and crustal thickness calculated for the entire melting regime is the average of the boxes in the RMC, and are shown at the lower right. After Plank and Langmuir [1992]. 
Because the AFM and batch equations give similar results, however, the concentration of the pooled fractional melt $\left(28^{*} \mathrm{C}_{\mathrm{o}}\right)$ is very similar to that of the pooled equilibrium melt $\left(23 * \mathrm{C}_{0}\right)$.

Fractional melting should also lead to lower maximum (and mean) extents of melting for the same mantle temperature, because $\gamma$ should be lower (as discussed above). For the sake of illustration, however, the fractional melting regime in Figure $34 b$ was constructed for the same $\gamma$ as for the equilibrium case. If a lower $\gamma$ is adopted (as in some examples below), then the maximum extent of melting will be less than $6 \%$, and the mean will be less than $3 \%$, for the same total pressure interval. These effects also lead to less crust produced during each increment of spreading.

To summarize, fractional melting should lead to roughly twice the mean pressure of melting, lower mean extent of melting, greater incompatible element concentrations, and less crust than equilibrium melting for the same mantle temperature.

\subsubsection{A Continuous Melting Regime}

Although we presented a simple incremental model for the sake of illustration, the same principles apply to a continuous melting regime. Figure 35 shows a melting regime and RMC generated upon comer flow, where $F$ is contoured linearly though the melting regime as $1.2 \% / \mathrm{kbar}$ (a good approximation to equations 19 and 20). Also shown is the distribution of melts that are extracted from the melting regime at steady-state as a function of depth. This melt distribution is the complement to the RMC. In Figure 35, the mass of melt $\left(M_{m}\right)$ created at each depth is linearly related to both $F$ and $P$. For example, 20 times more melt is produced by $20 \%$ melting at $1.67 \mathrm{kbar}$ than is produced by $1 \%$ melting at around $18 \mathrm{kbar}$. Mixing this linear distribution of melts together produces an oceanic crust with a characteristic composition and volume, as calculated by $K L 87$ and $M B 88$. This linear type of mixing results from linear melting contours with depth in the melting regime, and constant horizontal velocity outside the melting regime. One can imagine non-linear cases, however, that could result from more rapid flow through the shallow part of the melting regime, or non-linear melting contours [Plank and Langmuir, 1992].

In integral form, $\overline{\mathrm{F}}$ and $\overline{\mathrm{P}}$ are defined as:

$$
\begin{aligned}
& \bar{F}=\frac{\int_{0}^{F_{\max }}\left[M_{m}(F)\right] d F}{\int_{0}^{F_{\max }}\left[M_{s}(F)\right] d F} \\
& \bar{P}=\frac{\int_{P_{f}}^{P_{o}}\left[P M_{m}(P)\right] d P}{\int_{P_{f}}^{P_{0}}\left[M_{m}(P)\right] d P}
\end{aligned}
$$

where $M_{s}$ is the mass of solid prior to melting, $P_{0}$ is the pressure of intersection of the solidus, and $P_{f}$ is the final pressure. Because $F$ is defined as $M_{m} / M_{s}$, equation 24 becomes:

$$
\bar{F}=\frac{\int_{0}^{F_{\max }}\left[M_{m}(F)\right] d F}{\int_{0}^{F_{\max }}\left[M_{m}(F) / F\right] d F}
$$

RMC

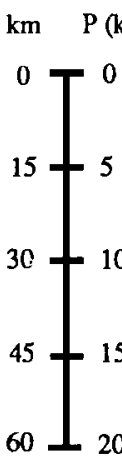

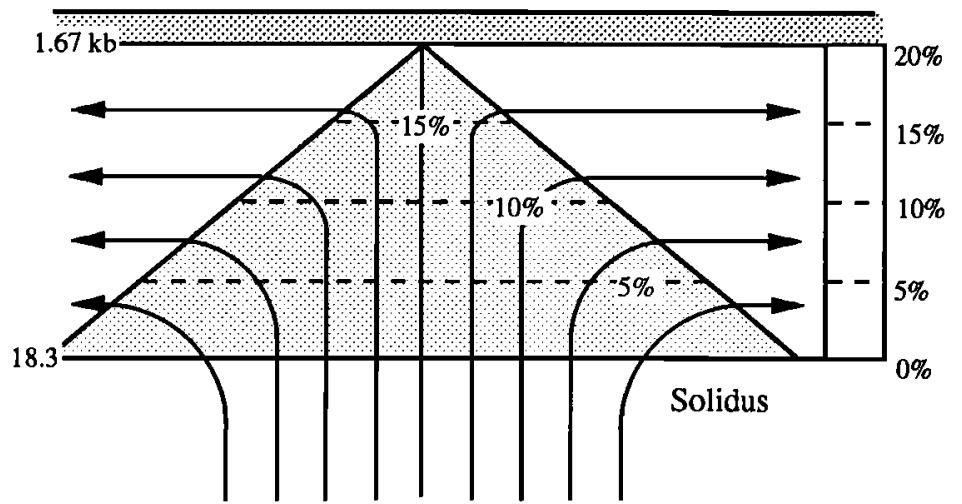

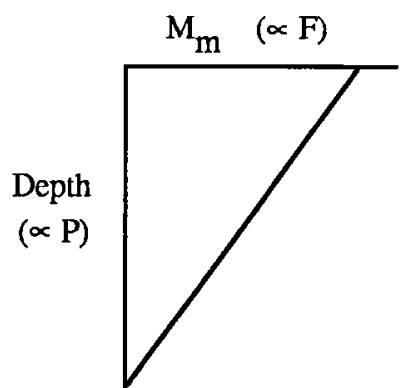

$$
\begin{gathered}
\overline{\mathrm{F}}=\mathrm{F}_{\max } / 2=10 \% \\
\overline{\mathrm{P}}=\left(\mathrm{P}_{\mathrm{o}}+2 \mathrm{P}_{\mathrm{f}}\right) / 3=7.2 \mathrm{~kb} \\
\mathrm{~h}_{\mathrm{c}}=\left(\mathrm{P}_{\mathrm{o}}-\mathrm{P}_{\mathrm{f}}\right) \overline{\mathrm{F}}=1.67 \mathrm{~kb}(\sim 5.5 \mathrm{~km})
\end{gathered}
$$

Fig. 35. As in Figure 34, but for a continuous melting regime where $\gamma$ is $1.2 \% / \mathrm{kbar}$. Also shown is the distribution of melt as a function of depth. The mass of melt $\left(\mathrm{M}_{\mathrm{m}}\right)$ produced anywhere in the melting regime is a linear function of both $F$ and $P$ because $\gamma$ is constant in this model. The steady-state oceanic crust produced from this melting regime has a $\bar{F}$ of $10 \%, \overline{\mathrm{P}}$ of $7.2 \mathrm{kbar}$, and is about $5.5 \mathrm{~km}$ thick. 
For linear mixing (Figure 35), $\mathrm{M}_{\mathrm{m}}=\mathrm{aF}$ ( $\mathrm{a}$ is a constant), and $\overline{\mathrm{F}}$ $=1 / 2 F_{\max }$ (integrating equation (26)). This is a general result of any linear mixing model, and is the same as in the incremental model above, and the same as presented in $K L 87$. Thus, although the maximum amount of melting beneath a ridge may be $20 \%$, the mean amount of melting reflected in the composition of the oceanic crust will be $10 \%$. If $\mathrm{M}_{\mathrm{m}}$ and $\mathrm{P}$ are also linearly related such that $M_{m}=b-c P$ (where $b$ and $c$ are constant), then $\bar{P}=\left(P_{o}+2 P_{f}\right) / 3$ (from equation 25). Thus $\bar{P}$ is shallower than half the pressure interval (it equals the upper third), reflecting the greater amount of melt generated at shallower pressures in the melting regime.

The chemical composition for the mean melts coming from the melting regime can be calculated by integrating the total liquid produced. This equation (from $K L 87$ ) is:

$$
\bar{C}_{i}=\frac{\int_{0}^{F_{\max }}\left[C_{i}(F) * F\right] d F}{\int_{0}^{F_{\max }} F d F}
$$

For a perfectly incompatible element, the liquid composition is simply $C_{o} / \bar{F}$. For most incompatible trace elements, the abundance calculated using a simple melting equation and $\overline{\mathrm{F}}$ will be a very good approximation.

The integrals above are general, and can be used to calculate the mean properties of the ocean crust given any distribution of melt in a melting regime. Because the mean extent of melting and mean pressure of melting are reflected in the composition of the oceanic crust (e.g., in its $\mathrm{Na}_{2} \mathrm{O}$ and $\mathrm{FeO}$ contents, respectively), we can use major element compositions to invert for melt distributions in the melting regime. Thus, coupling chemical data with measurements of the thickness of the oceanic crust (the total melt volume produced) provides powerful constraints on the melting processes beneath ridges.

\subsubsection{Many Shapes Lead to the Same RMC at Steady-State}

An important aspect of the mean extent and pressure of melting is that they are independent of the "shape" of the melting regime for a given pressure of intersection of the solidus, provided the melting contours are controlled by the amount of pressure release above the solidus, and there is no downward component of mantle flow within the melting regime (Plank and Langmuir, 1992). This can be simply illustrated by Figure 36, which shows two melting regimes with different shapes. The two shapes are produced by different mantle upwelling patterns, the curved shape by upwelling that decreases in velocity outward from the central axis, and the triangular shape by constant and rapid upwelling velocity. The horizontal velocity of the mantle that emerges out of both melting regimes is the same and equals the spreading rate. Because the flow of mantle out of the melting regime controls how much mantle may be processed through the melting regime, both shapes actually process the same amount of mantle during each increment of spreading. The flux of material (shaded) through both melting regimes is the same, and the different shapes are exactly balanced by the different upwelling patterns. Thus, although they have different shapes, both melting regimes lead to the same RMC, the same thickness of ocean crust, and the same mean extents of melting. This is a steady-state model; a new section of crust and residual mantle are produced during each increment of spreading.

The result that both shapes lead to the same RMC may seem counter-intuitive upon first glance at the problem. For example, the curved shape appears to contain a very large region of mantle that melts less than $5 \%$, and one might think

\section{Melting Regime Shape 1}

\section{Melting Regime Shape 2}

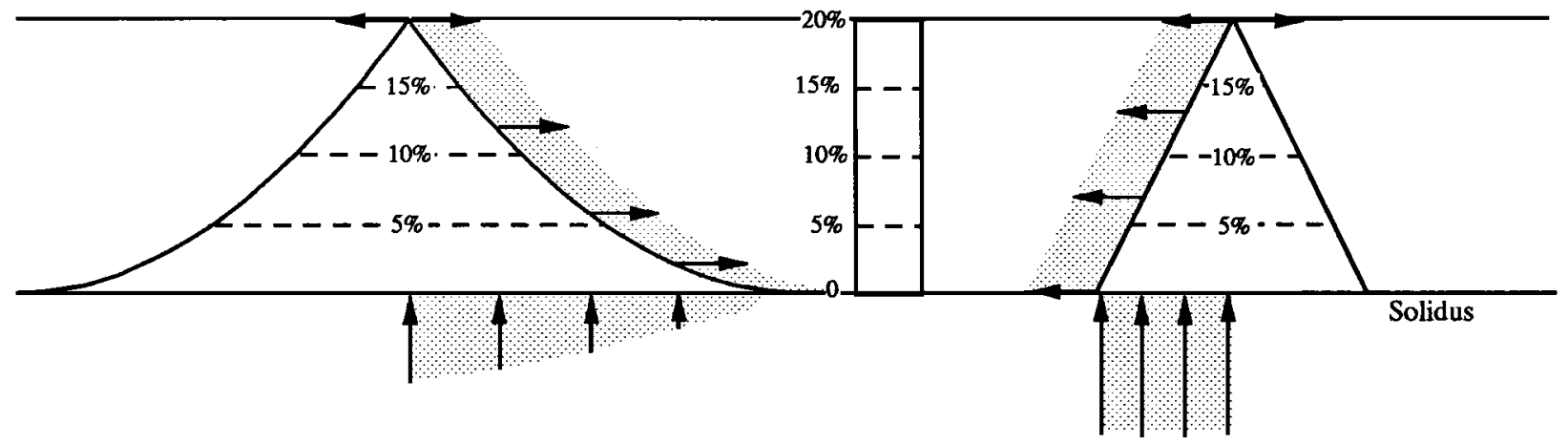

Fig. 36. The effect that two different shapes of the melting regime have on the residual melting column. Because the

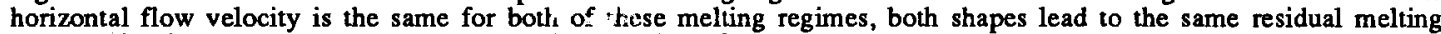
column (RMC), and thus the same volume and comipusition for the pooled melt. 
this would lead to lower mean extents of melting than for the triangular shape. But the current volume oi the melting regime is not the same as the amount of mantle passed through each part of the melting regime per spreading increment. Upwelling velocities are controlled by the slope of the upper boundary of the melting regime, and the upwelling velocity compensates for the variations in shape. Since melting beneath ridges is ultimately linked to spreading, the ocean crust results from the amount of mantle that is processed through the melting regime for each increment of spreading, and not simply the total volume of mantle that makes up the melting regime.

Although many shapes lead to the same RMC, different mixing functions can result from complications such as active upwelling and differential melt extraction from different parts of the melting regime. These effects have chemical consequences that can lead to different relationships between chemistry and crustal thickness. These more complex mixing functions will be considered in section 6.1, when their theoretical consequences can be quantitatively compared to the observed data.

\subsection{The Chemistry Function}

The melting function gives the extent of melting with distance above the solidus, the mixing function gives the relative amounts of each extent of meiting present in the melting regime. In order to calculate crustal compositions, we need to know the chemical compositions of the various melts produced within the melting regime.

\subsubsection{Experimental Constraints on the Compositional Variations Caused by Partial Melting of tine Mantle}

A function that describes the liquid composition during partial melting of the mantle must ultimately come from experimental data. Conceptually, this is a simple problem. A peridotite sample is loaded into an experimental capsule, heated and compressed to the temperature and pressure of interest, then quenched and analyzed. In practice, technical difficulties limit the experimentalist to working only at moderate pressures and with large melt fractions in the charge. Existing data provide quite complete information on moderate extents of melting [e.g., Jaques and Green, 1980; Stolper, 1980; Fujii and Scarfe, 1985; Falloon et al., 1988; Takahashi and Kushiro, 1983; Takahashi, 1986]. But there are virtually no data for the relatively small extents of melting that may be so important for understanding melting processes beneath the ridge. This gap in the experimental data is particularly troublesome in view of the evidence that at small extents of melting, volatile species may play an inportant role in the melting process, and volatile-bearing experiments are lacking. Nonetheless, the experimental data that do exist allow many important conclusions to be drawn.

It has been known for more than twenty years that basalts formed at greater pressures are more alkalic, silica undersaturated, and olivine normative than those formed at lower pressures [e.g., Green and Ringwood, 1967]. The interpretation of more recent experimental data over the years has been somewhat encumbered by the phase diagram approach, where the focus has been on finding isobaric pseudoinvariant points, rather than illustrating the actual compositional changes that are occurring as a function of pressure and extent of melting. When one does examine the experimental data element by element, some simple systematics emerge [e.g., Jaques and Green, 1980; Fujii and Scarfe, 1985; KL87].

Figures 37 and 38 present some of the compositional changes that occur during mantle melting, based on the peridotite melting experiments of Jaques and Green [1980], Fujii and Scarfe [1985], and Falloon and Green [1987]. Figure 37 shows the compositional variations with temperature at 10 kbar for four different peridotite compositions, which include different experimental configurations run in different laboratories. While there are offsets due to differences in the starting compositions, the qualitative trends for the various elements are consistent among the different sets of experiments. For example, although the absolute $\mathrm{Na}_{2} \mathrm{O}$ and $\mathrm{Al}_{2} \mathrm{O}_{3}$ contents of the melts are sensitive to the starting material, all the melting trends for these elements decrease with increasing T. Figure 38 shows the melting trends as a function of $F$ for a single peridotite composition at three different pressures [from Jaques and Green, 1980]. By combining the information given in Figures 37 and 38, and examining the results of other melting experiments, the systematics for each of the major elements as a function of extent and pressure of melting can be described.

$\mathrm{Na}_{2} \mathrm{O}$, as well as $\mathrm{TiO}_{2}$ and $\mathrm{K}_{2} \mathrm{O}$ (not shown), behave incompatibly during melting. Their concentrations in the melt are greatest at low $\mathrm{F}$ and decrease with increasing $\mathrm{F} . \mathrm{MgO}$ and $\mathrm{Al}_{2} \mathrm{O}_{3}$ also show strong variations with $\mathrm{F} . \mathrm{Al}_{2} \mathrm{O}_{3}$ decreases while $\mathrm{MgO}$ increases in the melt with increasing $\mathrm{F}$. There are also small pressure dependencies on $\mathrm{Al}_{2} \mathrm{O}_{3}$ and $\mathrm{MgO}$ concentrations at a given $\mathrm{F}$. $\mathrm{SiO}_{2}$ and $\mathrm{FeO}$ show marked pressure dependences. Higher $\mathrm{FeO}$ and lower $\mathrm{SiO}_{2}$ melts are produced at higher pressures. On the other hand, the effect of progressive melting at a single pressure is relatively small for both $\mathrm{SiO}_{2}$ and $\mathrm{FeO}$. $\mathrm{SiO}_{2}$ increases slightly with melting. $\mathrm{FeO}$ changes little, although its trend siows a kink when clinopyroxene disappears as a residual phase (near 25\% melting). $\mathrm{CaO}$ variations during melting are complex, but there is good agreement between different sets of experiments; $\mathrm{CaO}$ contents in the melt increase steadily upon melting, reach a maximum when clinopyoxene is consumed (near $25 \%$ melting), and then decrease upon continued melting.

Many of these qualitative trends in the major element contents of the melt can be understood by considering the mineralogical changes that occur during melting of peridotite. For example, $\mathrm{CaO}$ contents are strongly linked to the melting behavior of clinopyroxene. For a given pressure, the initial melt occurs at the lowest temperature, where partition coefficients for $\mathrm{Ca}$-bearing components are highest. As the temperature increases, clinopyroxene dissolves into the melt whenever $\mathrm{Ca}$ decreases below its equilibrium value. At the same time, the $K_{d}$ is decreasing with increased temperature, and 


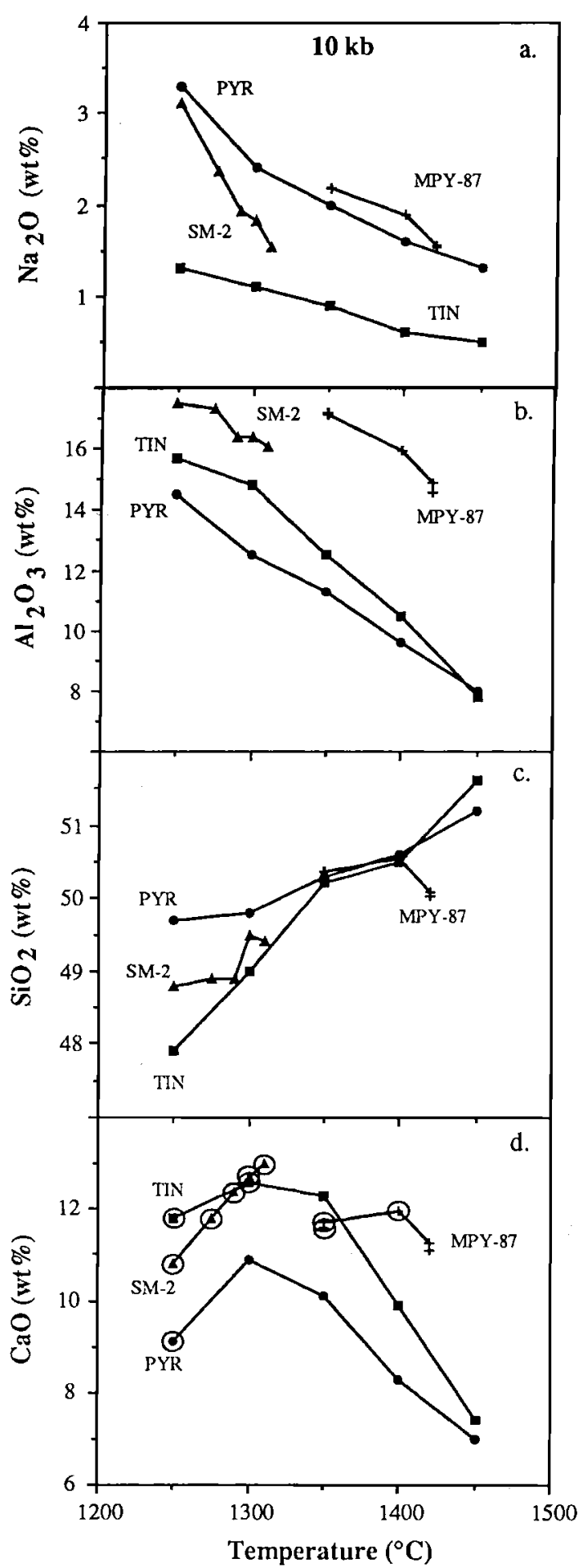

Fig. 37. Compositions of melts produced by melting of peridotite at $10 \mathrm{kbar}$ as a function of temperature of the experiment for four different starting compositions. Solid circles are Hawaiian Pyrolite (PYR) from Jaques and Green [1980]; solid squares are depleted Tinaquillo lherzolite (TIN) from Jaques and Green [1980]; solid triangles are SM-2 from $F$ ujii and Scarfe [1985]; and crosses are MORB Pyrolite sandwich experiments with about 10\% basalt (MPY-87) from Falloon and Green [1987].

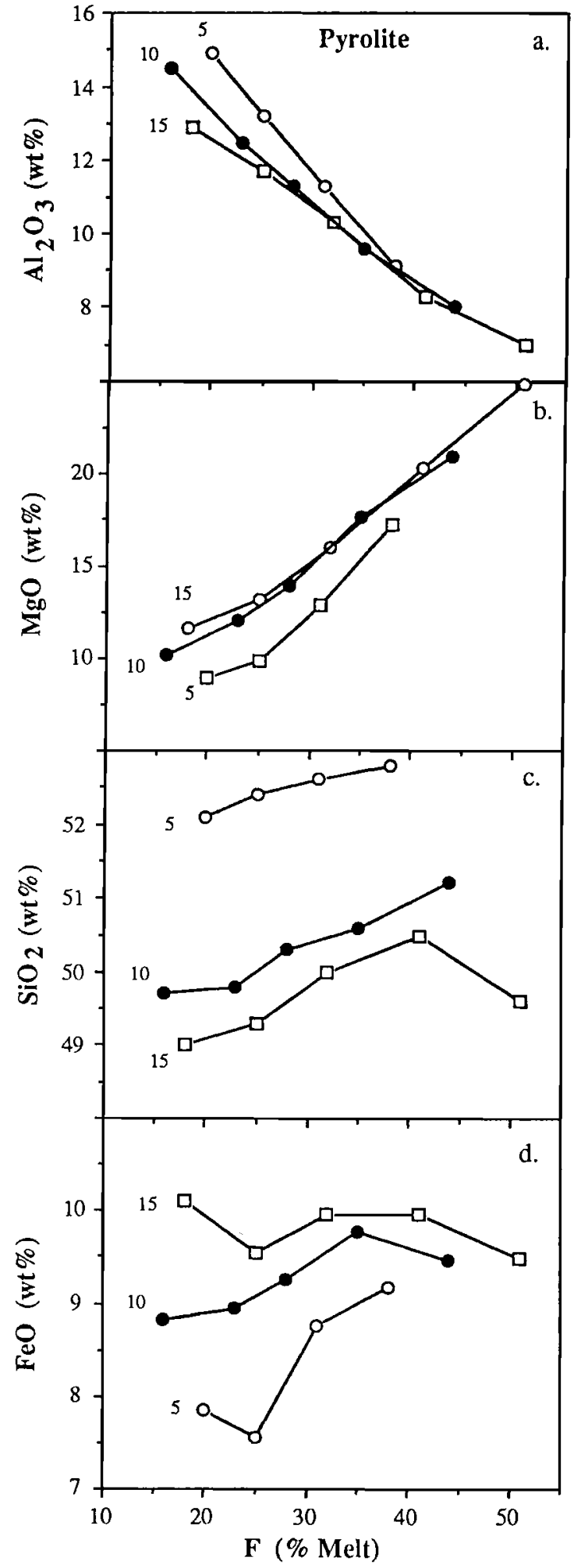

Fig. 38. Extent of melting $(\% \mathrm{~F})$ vs. the compositions of melts produced by melting of peridotite of pyrolite composition at $5 \mathrm{kbar}$ (open circles), $10 \mathrm{kbar}$ (solid circles), and $15 \mathrm{kbar}$ (open squares). Data from Jaques and Green [1980]. 
therefore the equilibrium content of $\mathrm{CaO}$ in the liquid increases. Thus $\mathrm{CaO}$ increases as long as clinopyroxene is available to contribute more $\mathrm{CaO}$ to the liquid. With the disappearance of clinopyroxene, $\mathrm{CaO}$ behaves incompatibly and is diluted by the addition of Ca-poor phases (olivine, orthopyroxene, spinel) to the melt.

$\mathrm{Al}_{2} \mathrm{O}_{3}$ decreases with melting because of the progressive melting of the aluminous phases, the progressive melting of clinopyroxene, and the progressive change in the spinel composition from Al-rich to $\mathrm{Cr}$-rich. The small decrease in $\mathrm{Al}_{2} \mathrm{O}_{3}$ contents of the melt with increasing pressure is a consequence of more aluminous pyroxene compositions at higher pressures. The $\mathrm{MgO}, \mathrm{FeO}$ and $\mathrm{SiO}_{2}$ systematics are controlled largely by olivine partitioning relationships, as discussed in more detail below.

In addition to the qualitative trends indicated in the peridotite melting experiments, additional constraints on the melting behavior of the mantle come from combining experimental data with simple partitioning models. The partitioning models provide a means of extending the experimental data between and beyond the pressures, temperatures and compositions of the experiments themselves. For example, $\mathrm{Na}, \mathrm{Ti}$ and $\mathrm{K}$ can be modeled as trace elements during batch or fractional melting, given information on their partition coefficients.

Since $\mathrm{Na}_{2} \mathrm{O}$ plays a central role in our discussions, it is important to consider the constraints on its behavior. The $\mathrm{Na}_{2} \mathrm{O}$ contents of olivine, orthopyroxene and spinel are almost zero, so the behavior of $\mathrm{Na}_{2} \mathrm{O}$ during melting of spinel peridotite is controlled by the partition coefficient for $\mathrm{Na}$ between clinopyoxene and liquid, and by the relative abundance of clinopyroxene in the solid residue. Partitioning data for $\mathrm{Na}$ in clinopyroxene from various experimental studies at pressures up to $30 \mathrm{kbar}$ are shown in Figure 39. The partition coefficient appears to increase with increasing temperature because of the increased jadeite component in residual clinopyroxene at higher pressures (and hence temperatures). The $\mathrm{Na} \mathrm{K}$ is on the order of 0.2 for temperatures and pressures appropriate to most MORB. Since clinopyroxene makes up less than $20 \%$ of the mantle starting composition, the $\mathrm{D}$ for $\mathrm{Na}$ between solid and liquid begins at a value of less than 0.04, and continually decreases as melting proceeds and clinopyroxene is preferentially consumed by the melt. Thus $\mathrm{Na}$ behaves essentially as an incompatible trace element during melting of mantle peridotite. Similar approaches can be used to model $\mathrm{Ti}$ and $\mathrm{K}$. Quantitative evaluation of $\mathrm{Ti}_{\mathrm{d}} \mathrm{s}$ is given in Appendix A.

A second quantitative constraint comes from the very regular partitioning relationships for $\mathrm{FeO}$ and $\mathrm{MgO}$ between olivine and liquid, originally shown by Roeder and Emslie [1970]. The distribution coefficient for the exchange of $\mathrm{Fe}$ and $\mathrm{Mg}$ between olivine and melt $\left[\mathrm{K}_{\mathrm{D}}\right.$; equation 12] is close to 0.3 . Although there are slight temperature and pressure dependencies to this value [e.g., Bender et al., 1978], the effects of increasing both temperature and pressure are compensatory, so that the value of $K_{\mathrm{D}}$ along the solidus remains close to 0.3 . Olivine is always present during mantle melting, for it is the last phase to melt, and this fact, combined with the relatively constant $K_{D}$,

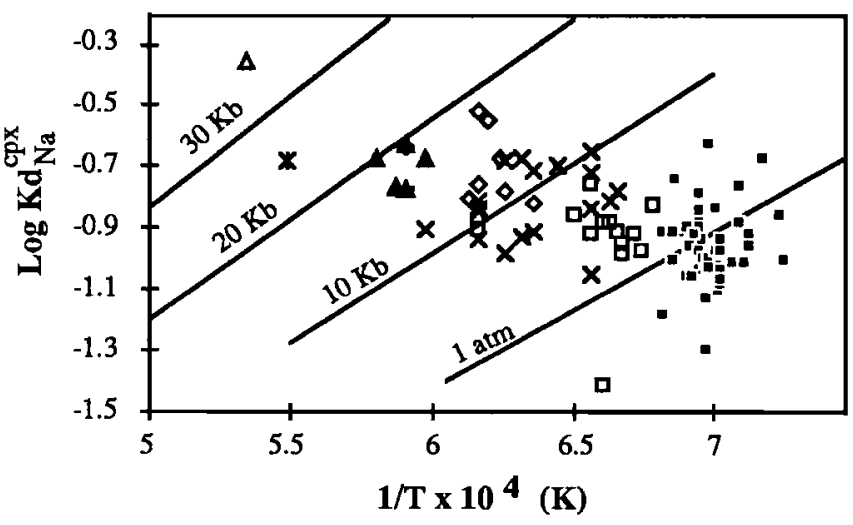

Fig. 39. Clinopyroxene melt $\mathrm{K}_{\mathrm{d}}$ for $\mathrm{Na}$ at variou i pressures vs. inverse temperature for experiments on mafic systems. Data are from Walker et al., [1979]; Elthon and Scarfe [1984]; T. Falloon, pers. comm. [1990]; Grove and Bryan [1983]; Grove et al., this volume; Tormey et al. [1987]; some experiments reported in Nielson and Drake [1979]; Bender et al. [1978]. The different pressures are indicated by different symbols: small closed squares, 1 atm; oper, squises, $8 \mathrm{kbar}$, $x$ 's, 10 kbar; open diamonds, 15 kbar; solid triangles, $20 \mathrm{~kb}$.1r; $\mathrm{X}$ with vertical slash, $25 \mathrm{kbar}$; and open triangle, $30 \mathrm{kbar}$. The cirves were calculated from the equations given in Appendix $A$ for the $K_{d}$ 's as a function of temperature and pressure. Each curve was calculated for different temperatures at a single pressure. Note the large pressure dependence for $\mathrm{Na}$.

constrains the behavior of $\mathrm{Fe}$ and $\mathrm{Mg}$ during melting [Hanson and Langmuir, 1978; Langmuir and Hanson, 1980; Appendix B].

In fact, the strong pressure dependences of $\mathrm{FeO}$ and $\mathrm{SiO}_{2}$ shown in the experiments can be predicted simply from the behavior of olivine partition coefficients as a function of temperature and pressure. The $\mathrm{FeO}$ and $\mathrm{MgO} \mathrm{K}_{\mathrm{d}}$ 's for olivine increase with increasing pressure and with decreasing temperature. This produces competing effects on the olivine $\mathrm{K}_{\mathrm{d}}$ 's along the mantle solidus where pressure and temperature both increase. To determine whether the $P$ or $T$ effect dominates, the relative magnitude of their respective effects on the olivine $K_{d}$ 's must be considered. The pressure effect on the olivine $K_{d}$ 's is on the order of $5 \% / \mathrm{kbar}$, while the mantle solidus is on the order of $12 \% \mathrm{kbar}$ (see Appendix B for references). Thus, upon higher pressure melting of the mantle, the temperature effect on the olivine $K_{d}$ s dominates, and the $\mathrm{Mg}$ and $\mathrm{Fe} \mathrm{K}_{\mathrm{d}}$ s are lowered. This means that the melt is richer in olivine components (higher $\mathrm{Fe}$ and $\mathrm{Mg}$ and lower $\mathrm{Si}$ ) at higher pressures along the mantle solidus. This prediction based simply on the olivine $\mathrm{K}_{d}$ 's provides independent confirmation of the trends in the Jaques and Green experiments, which suffered significant Fe loss [Jaques and Green, 1980; Falloon and Green, 1988].

Thus the qualitative compositional trends indicated by the peridotite melting experiments combined with quantitative partitioning relationships provide important constraints on the systematics of mantle melting. Any successful melting model must take into account the following three constraints:

1. Na, Ti and $\mathrm{K}$ can be modeled as incompatible trace elements, and their melting behavior must be consistent with 


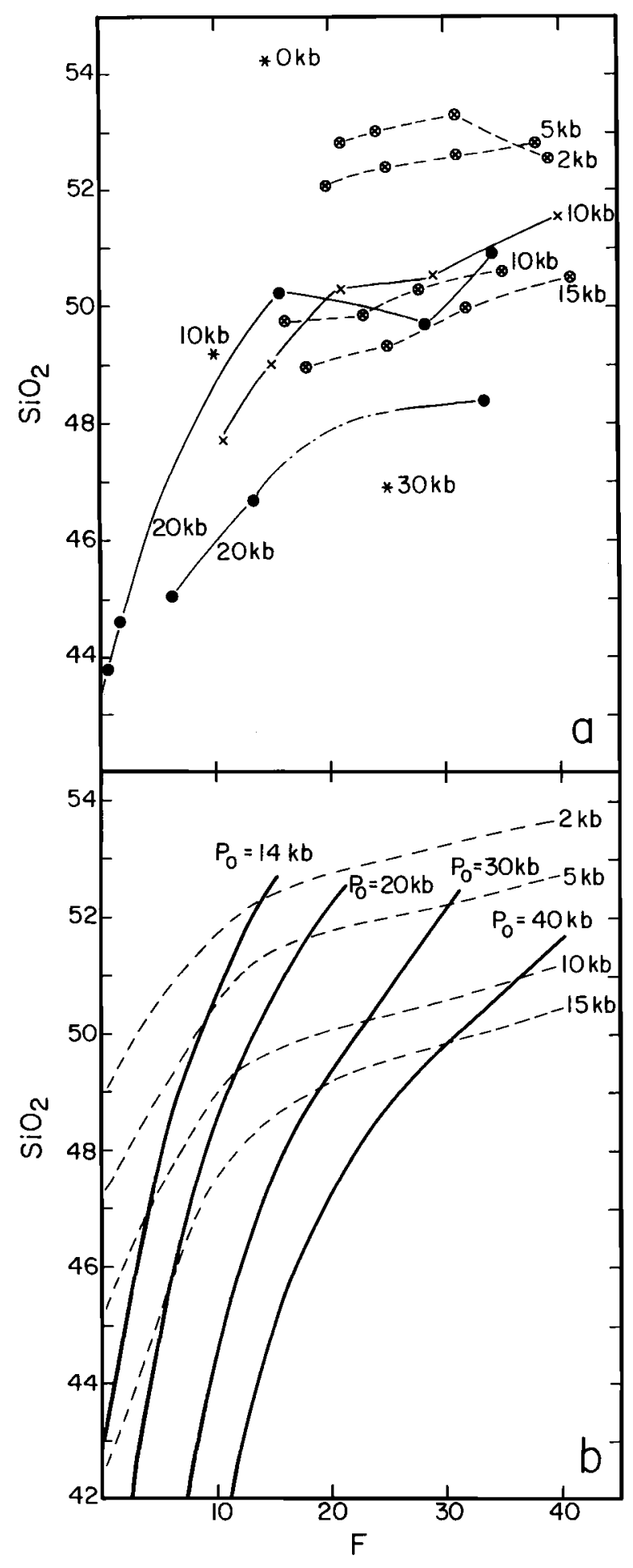

Fig. 40. $\mathrm{SiO}_{2}$ (wt\%) vs. $\mathrm{F}$ (extent of melting). a) Results of several experimental studies on the isobaric melting of various peridotitic compositions at the pressures indicated. Sources and starting compositions are: pyrolite (circled crosses), Tinaquillo lherzolite (crosses, only 10 kbar melts shown) [Jaques and Green, 1980]; gamet lherzolite +/-water (solid circles) [Mysen and Kushiro, 1977]; natural known clinopyroxene partition coefficients. The $\mathrm{D}$ for these elements decrease in the order $\mathrm{Ti}-\mathrm{Na}-\mathrm{K}$.

2. $\mathrm{FeO}$ and $\mathrm{SiO}_{2}$ contents of the melt are strongly controlled by the pressure of melting. $\mathrm{FeO}$ and $\mathrm{MgO}$ variations must be consistent with olivine partitioning, stoichiometry and mass balance. The $\mathrm{FeO} / \mathrm{MgO}$ ratio of the melt must be consistent with a $K_{D}$ of about 0.3 (equation 12).

3. $\mathrm{CaO}$ increases steadily with extent of melting as long as clinopyroxene remains in the solid residue, then decreases once clinopyroxene is consumed; this effect may vary with pressure, as the $\mathrm{CaO}$ contents of clinopyroxenes decrease with increasing pressure. $\mathrm{Al}_{2} \mathrm{O}_{3}$ contents of the melt decrease with increasing extent of melting. Thus the $\mathrm{CaO} / \mathrm{Al}_{2} \mathrm{O}_{3}$ ratio increases with progressive melting as long as clinopyroxene is a residual phase.

\subsubsection{A Critical Evaluation of Published Chemistry Functions}

There have been a number of approaches to calculating mantle melt compositions during adiabatic ascent: Langmuir and Hanson [1980 (FeO and $\mathrm{MgO}$ only)], KL87, McKenzie and Bickle, [1988], and subsequent to the short course, Niu and Batiza [1991]. In the following section, we evaluate these melting models in light of the qualitative guidelines given above. We then present in section 5 a revised $K_{d}$ approach for four elements, $\mathrm{Fe}, \mathrm{Mg}, \mathrm{Na}$ and $\mathrm{Ti}$, which is fully quantitative and has several advantages over previous approaches.

$K L 87$ calculated the concentrations of each oxide in the melt as a function of extent and pressure of melting, based on available experimental data and theoretical constraints. The abundances of different elements were calculated in different ways, depending on the information and theory available. For several elements $\left(\mathrm{SiO}_{2}, \mathrm{CaO}, \mathrm{Al}_{2} \mathrm{O}_{3}, \mathrm{FeO}\right)$, smoothed curves were fit to the experimental melt compositions as a function of extent and pressure of melting. An example is shown in Figure $40 a$ for $\mathrm{SiO}_{2} . \mathrm{SiO}_{2}$ decreases with the pressure of melting and increases with the extent of melting. There is little experimental data to predict melt compositions at low extents of melting, and the data that do exist have experimental difficulties; nevertheless, the information available suggests that $\mathrm{SiO}_{2}$ is low in melts produced by less than about $10 \%$ melting [c.f., Falloon et al., 1988]. Using the general trends inferred from the experimental data as a guide, smoothed isobaric melting paths were drawn through the data (dashed lines in Figure 40b). The estimated $\mathrm{SiO}_{2}$ content for any pressure and extent of melting can thus be read off the graph in Figure $40 b$.

spinel therzolite (asterisks; extents of melting estimated from reported $\mathrm{Na}_{2} \mathrm{O}$ and $\mathrm{TiO}_{2}$ abundances) [Takahashi, 1986]. b) From the trends of the experimental data shown in Panel (a), with particular emphasis on the pyrolite melting studies, isobaric melting curves (dashed) were estimated for $2,5,10$, and $15 \mathrm{kbar}$. Assuming $1.2 \%$ melt $/ \mathrm{kbar}$ pressure release, polybaric melting curves (solid) were estimated for $\mathrm{P}_{0}$ of 40 , 30,20 , and $14 \mathrm{kbar}$, by fitting to the isobaric melting curves (e.g., for $P_{0}=40,30 \%$ melting will be achieved by $15 \mathrm{kbar}$ ). The polybaric melting curves estimate the $\mathrm{SiO}_{2}$ abundances in instantaneous melts present in the melting column at each pressure above the solidus. Note that $\mathrm{SiO}_{2}$ contents at low extents of melting are unconstrained, and are probably too low for dry melting. From Klein and Langmuir [1987]. 
For those elements that behave as incompatible trace elements during mantle melting of spinel lherzolite $\left(\mathrm{Na}_{2} \mathrm{O}\right.$, $\mathrm{TiO}_{2}$ ), concentrations were calculated using partition coefficients consistent with peridotite melting experiments (e.g., Figure 39), and source compositions appropriate to the MORB mantle. $\mathrm{MgO}$ contents were constrained by the $\mathrm{FeO}$ contents using the method of Langmuir and Hanson [1980].

The $K L 87$ chemistry function meets the three experimental constraints listed above, but suffers from being semiquantitative and difficult for other investigators to reproduce.

McKenzie and Bickle [1988] presented a series of equations to parameterize experimental melt compositions. Instead of $F$, they defined a new parameter 'T', with which to parameterize the experimental data. $T^{\prime}$ is temperature normalized to the temperature interval between the solidus and liquidus of average peridotite, at the same pressure. By defining $T^{\prime}$, they were able to use virtually all experimental data, including experiments run on different starting compositions or experiments for which $\mathrm{F}$ was not determined. $M B 88$ related $\mathrm{T}$ " to $\mathrm{F}$ by fitting a polynomial function to the limited number of experiments in which $\mathrm{F}$ had been determined.

Figure $41 a$ shows experimental melt compositions plotted as a function of $\mathrm{T}^{\prime}$, as well as the paramterization determined by $M B 88$ to fit the data. For $\mathrm{FeO}$ and $\mathrm{MgO}$, separate parameterizations were determined for 0,10 and $20 \mathrm{kbar}$. $\mathrm{SiO}_{2}$, calculated by difference from $100 \%$, also shows a pressure dependence due to the pressure dependencies of the other parameters. These parameterizations express elemental variations as a function of inferred extent of melting at a given pressure, and are thus analogous, although by no means identical in form, to the isobaric melting paths estimated by $K L 87$ (e.g., Figure 40a).

In spite of the large experimental data base used by $M B 88$, and the quantitative nature of their parameterizations, their results do not produce improved representations of the chemical variations that occur during mantle melting. Indeed, one might argue that it is because $M B 88$ used all experimental data and approached the data in a wholly statistical way that their isobaric melting curves have inherent problems. The problems are particularly apparent for $\mathrm{CaO}, \mathrm{FeO}$ and $\mathrm{Na}_{2} \mathrm{O}$.

Figure 41 shows the experimental data for $\mathrm{CaO}$ and the preferred $M B 88$ curve. Over a range of $\mathrm{T}^{\prime}$ from -0.5 to -0.2 (corresponding to $0 \%$ to $34 \%$ melting), the original parameterization curve has a negative slope, which contradicts one of the constraints obtained from careful examination of the experimental data. The overlap of the various experiments prevents the data set as a whole from showing the positive slopes apparent for individual groups of experiments. Indeed, when they compared their results for $\mathrm{CaO}$ to the MORB data, the observed lack of agreement led them to add an arbitrary correction factor (a "pressure dependence") to the parameterization.

A second problem in the parameterization of $M B 88$ concerns FeO. $M B 88$ recognized that both $\mathrm{FeO}$ and $\mathrm{MgO}$ show strong pressure dependencies. However, their pressure dependence for $\mathrm{FeO}$ in the preferred parameterization is very small, changing by just over $1 \mathrm{wt} \% \mathrm{FeO}$ over a pressure interval of $20 \mathrm{kbar}$ at
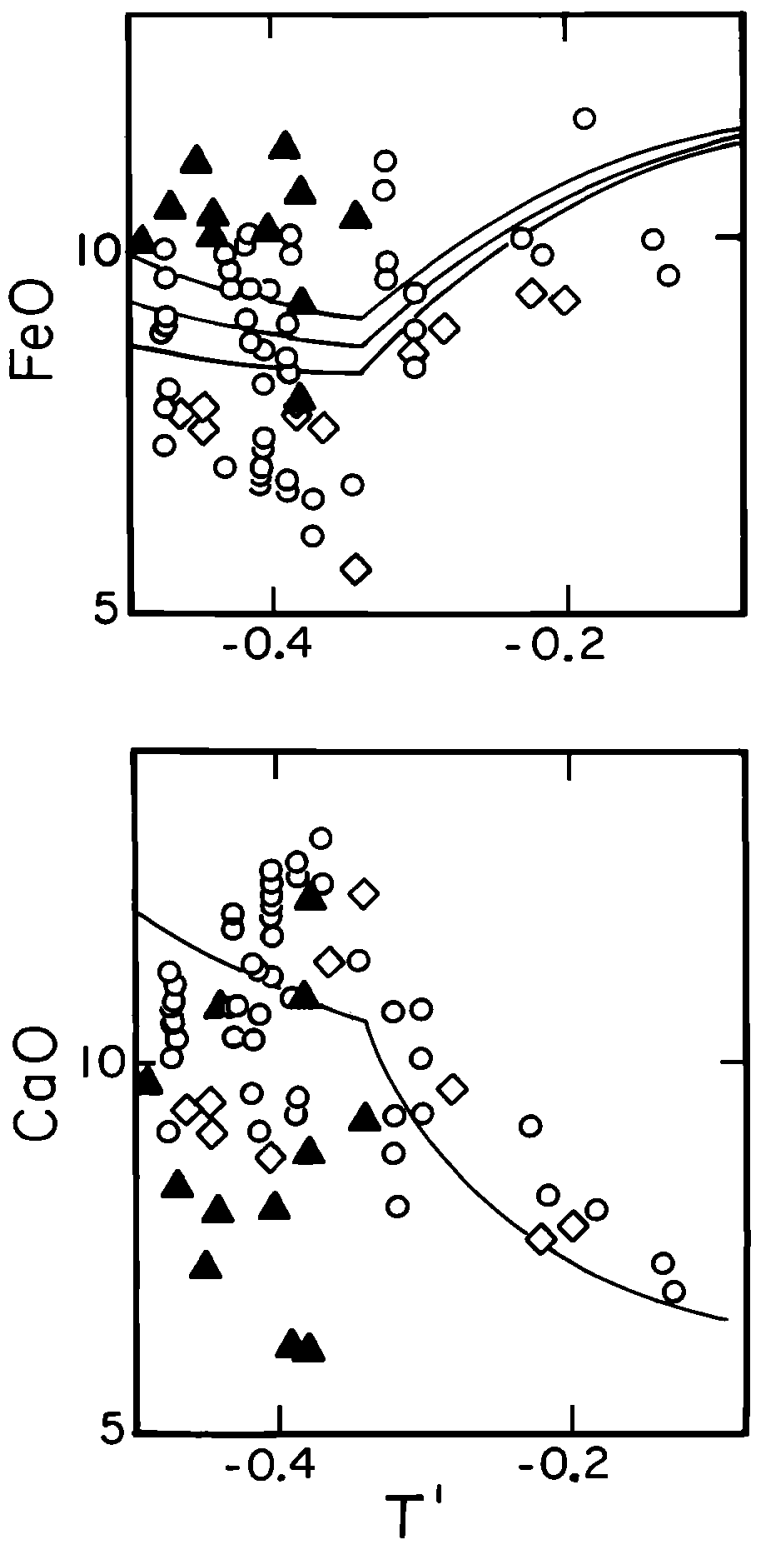

Fig. 41. Compositions of melts produced experimentally by melting of peridotite as a function of $\mathrm{T}$ " (which is related to extent of melting). Crosses mark compositions of experiments carried out at pressures $<$ $0.5 \mathrm{GPa}$, circles at pressures of $0.5<\mathrm{P}<1.5 \mathrm{GPa}$, and triangles $\mathrm{P}>1.5$ $\mathrm{GPa}$. Curves show parameterizations of the experimental melt compositions as a function of $T^{\prime}$; where three curves are shown, they are the calculated compositions at pressures 0 (not labelled), 1 and 2 GPa. After McKenzie and Bickle [1988]; see this reference for experimental data sources and explanation of ' $\mathrm{T}$ ".

$0 \%$ melting. Figure 41 shows that this change is too small to account for the data. The $M B 88$ parameterizations also do not satisfy the $K_{D}$ constraints for the partitioning of $\mathrm{FeO}$ and $\mathrm{MgO}$ between olivine and liquid (discussed above; equation 12). For example, their calculated normal MORB composition yields a $\mathrm{K}_{\mathrm{D}}$ of 0.24 for equilibrium with an $\mathrm{Fo}_{90}$ olivine, which is far 
outside the experimental limits of $0.3 \pm 0.03$ (but see below for an improvement by Watson and McKenzie, [1991].

A third problem concerns $\mathrm{Na}_{2} \mathrm{O}$. The bulk distribution coefficient (D) for $\mathrm{Na}_{2} \mathrm{O}$ given by $M B 88$ is 0.169 , a value which exceeds by about a factor of four the $D$ permitted by the experimental data (from Figure 39, and assuming a maximum of $20 \% \mathrm{cpx}$ in the bulk mantle). The $\mathrm{D}$ also stays constant in the $M B 88$ calculations, instead of decreasing as clinopyroxene progressively melts out of the residue and the pressure decreases. The source concentration of $\mathrm{Na}_{2} \mathrm{O}$ used by $M B 88$ is $0.44 \mathrm{wt} \%$, while the source concentration for $\mathrm{Na}_{2} \mathrm{O}$ in the mantle is well constrained to be about $0.3( \pm 0.03) \mathrm{wt} \%$, based on data from primitive mantle nodules [Jagoutz et al., 1979], peridotite suites [Frey et al., 1985] and chondritic considerations [Hart and Zindler, 1986]. The combination of these factors creates serious discrepancies between the $M B 88$ $\mathrm{Na}_{2} \mathrm{O}$ calculation and that expected from more appropriate values of $D$ and source abundance, as illustrated in Figure 42. The $M B 88$ values are too low at low extents of melting, and too high at higher extents of melting. At high values their calculations violate mass balance, with more $\mathrm{Na}$ present in the liquid than is present in the initial source. Thus, while the calculations consistent with the experiments have $\mathrm{Na}_{2} \mathrm{O}$ vary by almost a factor of four from $0-20 \%$ melting, the $M B 88$ calculations produce variations of only $60 \%$.

Watson and McKenzie [1991] have recently presented a revised chemistry function. Their revised function has the same problems with respect to $\mathrm{CaO}$ and $\mathrm{Na}_{2} \mathrm{O}$, but has been made consistent with the olivine/liquid $\mathrm{K}_{\mathrm{D}}$ for $\mathrm{FeO}$ and $\mathrm{MgO}$.

The MB88 and Watson and McKenzie, [1991] approach has the advantage of being a quantitative parameterization that can

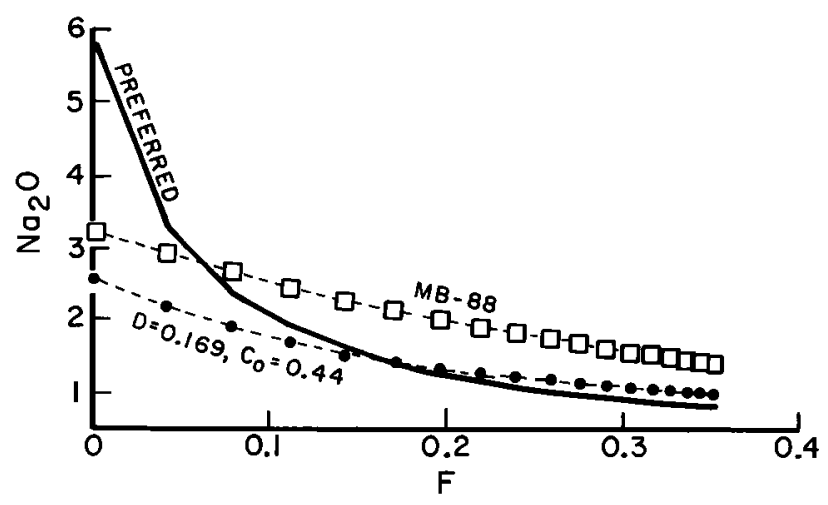

Fig. 42. Calculated wt\% $\mathrm{Na}_{2} \mathrm{O}$ in melts vs. extent of melting $(\mathrm{F})$. Open squares show the results using the parameterizations of McKenzie and Bickle [1988]. Filled circles are a calculation for equilibrium melting using a bulk $\mathrm{D}$ of 0.169 and $\mathrm{C}_{0}=0.44 \mathrm{wt} \% \mathrm{Na}_{2} \mathrm{O}$, the source abundance used by McKenzie and Bickle [1988]. The solid line shows a preferred equilibrium melting calculation that is consistent with the experimental data on $\mathrm{Na}$ partitioning, using a $\mathrm{C}_{0}$ of $0.3 \mathrm{wt} \% \mathrm{Na}_{2} \mathrm{O}$, a $\mathrm{K}_{d}$ for clinopyroxene of 0.15 (bulk $D$ of about 0.03 ), and assuming that clinopyroxene progressively melts out of the residue and is no longer a residual phase by $22 \%$ melting (thus, $\mathrm{D}$ is not constant). be put into a variety of models, and it should be able to be modified to produce chemical variations more consistent with the experimental constraints given above. In their current forms, however, these chemistry functions are not adequate for detailed modeling of mantle melting.

Niu and Batiza [1991, hereafter NB91] have recently presented a third approach to the determination of a chemistry function for mantle melting. Their chemistry function is based largely on the experiments of Jaques and Green [1980], although they applied corrections to the data in an effort of account for the systematic errors identified by Falloon and Green [1987; 1988]. NB91 extract apparent D's from the experiments using a mass balance equation. This enables them, in turn, to reproduce a given set of experiments quite well. From one set of experiments to another, however, the apparent bulk distribution coefficients change, so one set of values cannot be applied to a variety of potential mantle compositions.

Because a complete set of experiments on their preferred mantle composition does not exist, $N B 91$ manipulate the apparent D's in an effort to reproduce the limited experiments that do exist on this composition. This makes it difficult to evaluate the accuracy of the results outside the experimental range. The $N B 91$ parameterization for equilibrium melting, however, does reproduce the general trends of the experimental data, such as the changes of $\mathrm{Na}_{2} \mathrm{O}$ and $\mathrm{CaO}$ with $\mathrm{F}$. Therefore, the $N B 91$ approach should be able to effectively parameterize equilibrium melting where there is a complete set of experiments on a single bulk composition.

\subsection{Results of Previous Attempts to Combine Melting, Mixing and Chemistry Functions}

In sections $4.2,4.3$ and 4.4 above, we have described some of the reasoning and uncertainties in the development of the three functions necessary to calculate melt compositions produced during mantle upwelling beneath the ridge. In the following, we describe three previous attempts to combine melting, mixing and chemistry functions and compare these results to the MORB data set. We then present a new quantitative model for calculating mantle melt compositions.

Klein and Langmuir [1987] adopted a constant value for $(\partial \mathrm{F} / \partial \mathrm{P})$ of $1.2 \%$ melt/kbar, similar to the value used by Ahern and Turcotte [1979]. Their chemistry function, based on the results of equilibrium melting experiments at different pressures, required the assumption of batch melting during ascent. From the isobaric melting curves (e.g., dashed lines in Figure $40 \mathrm{~b}$ ), polybaric melting curves were calculated for different pressures of intersection of the mantle solidus (solid lines in Figure $40 b$ ). KL87 used a linear mixing function, where the polybaric melt compositions were weighted by $F$ (their equation 9). This pooled melt is the complement to the residual mantle column depicted in Figures 2 and 34.

The pooled melt compositions calculated in this way for different pressures of intersection of the mantle solidus result 
from different mantle temperatures. If these pooled melt compositions then crystallize at low pressure to $8 \mathrm{wt} \% \mathrm{MgO}$, their compositions can be compared to the MORB data. The chemical variations produced by different pressures of intersection of the mantle solidus (taller or shorter RMCs) result in an inverse correlation between $\mathrm{Fe}_{8.0}$ and $\mathrm{Na}_{8.0}$. Mantle that intersects the solidus deep produces an aggregate melt of high $\overline{\mathbf{F}}$ and $\overline{\mathbf{P}}$ (low sodium, high iron), while mantle that intersects the solidus shallow produces an aggregate melt of low $\overline{\mathrm{F}}$ and $\overline{\mathrm{P}}$ (high sodium and low iron; Figure $43 a$ ). In addition, the resulting pooled melt compositions form an inverse correlation between $\mathrm{Na}_{8.0}$ and $\mathrm{CaO} / \mathrm{Al}_{2} \mathrm{O}_{3}$. Thus, the model adopted by $K L 87$ reproduces the global vector of chemical variability in its overall trends. $K L 87$ also modeled the expected correlation between $\mathrm{Na}_{8.0}$ and crustal thickness (assuming two slightly different concentrations of $\mathrm{Na}_{2} \mathrm{O}$ in the source), and these calculations agree with the trend of the observations (Figure 44).

In detail, however, the calculated chemical variations show discrepancies with the observed global chemical variations. Compared to the global vector, the $K L 87$ model reproduces the observed global range in $\mathrm{Na}_{8,0}$, but only half the observed range in $\mathrm{Fe}_{8.0}$. Furthermore, the calculated melts are offset to lower $\mathrm{Fe}_{8.0}$ contents for the same $\mathrm{Na}_{8.0}$ (Figure 43a). Another important test is to see how well the $K L 87$ model reproduces normal ocean crust of average depth and crustal thickness, such as the East Pacific Rise between $8^{\circ} \mathrm{N}$ and $14^{\circ} \mathrm{N}$. The $K L 87$ calculated melt composition for $6 \mathrm{~km}$ crustal thickness is compared to EPR data in Figure $43 b$ where it can be seen that the calculated $\mathrm{Fe}_{8,0}$ value is significantly lower than observed in the EPR data. In addition, the calculated $\mathrm{Si}_{8.0}$ value is slightly high, despite the fact that $K L 87$ used very low $\mathrm{SiO}_{2}$ contents for low extents of melting (see Figure $43 a$ ), an aspect of their paper that has been criticized by Falloon et al. [1988].

In spite of these discrepancies, the general consistency of the calculated mean melt compositions and the observed regional averages suggests that the global vector results from regional variations in mantle temperature. The failure of the model to reproduce the observed variability in detail suggests that some aspect of the $K L 87$ model (chemistry, melting and/or mixing function) needs modification.

$M B 88$ combined their chemistry function (for equilibrium melting), with a non-linear melting function, and a linear mixing function. The $M B 88$ results approximate the MORB data, but do not reproduce the absolute values or slopes of the observations in detail (Figure 43). Their total range in $\mathrm{Na}$ is 1.6-2.7, only two-thirds of the observed range in $\mathrm{Na}_{8.0}$ values of 1.7-3.6. More importantly, all pooled melts with $>2.4 \mathrm{wt} \%$ $\mathrm{Na}_{2} \mathrm{O}$ produce less than $4 \mathrm{~km}$ of crust, while a pooled melt with $3 \mathrm{wt} \% \mathrm{Na}_{2} \mathrm{O}$, a common value for MORB, produces zero crustal thickness! (Figure 44) These problems can be traced directly to their chemistry function. As discussed above, the inappropriately high $\mathrm{D}$ value for $\mathrm{Na}_{2} \mathrm{O}$ results in too narrow a range in calculated $\mathrm{Na}_{2} \mathrm{O}$ contents. Secondly, without the use of the arbitrary pressure correction for $\mathrm{CaO}$ noted above, there is very little range in the calculated $\mathrm{CaO} / \mathrm{Al}_{2} \mathrm{O}_{3}$ ratios (their Figure $15 b$ ). Lastly, like the $K L 87$ results, the $M B 88$
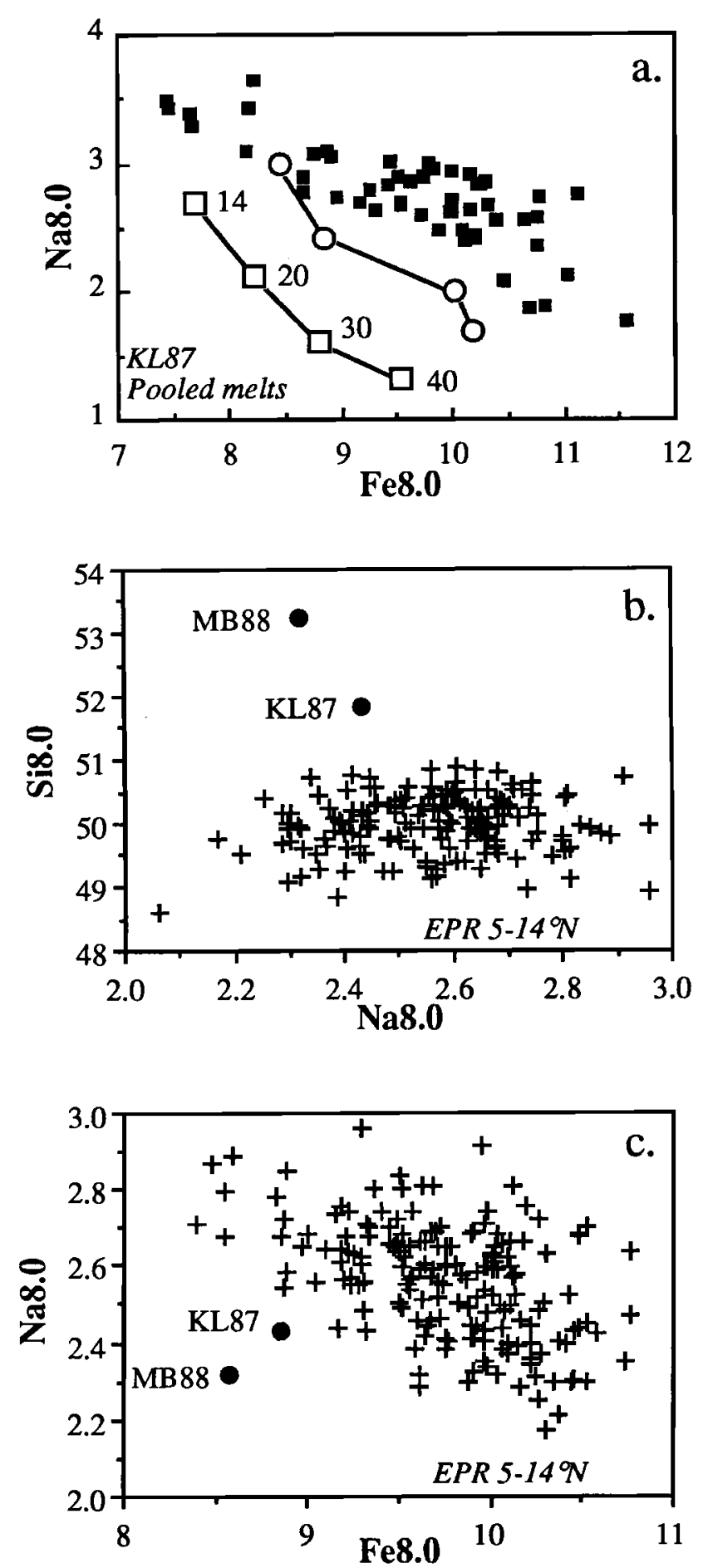

Fig. 43. $\mathrm{Na}_{8.0}$ vs. a) $\mathrm{Si}_{8.0}$ and b) $\mathrm{Fe}_{8.0}$ for samples from the East Pacific Rise between $5^{\circ} \mathrm{N}$ and $14^{\circ} \mathrm{N}$ (data as in Figure 24), and the mean melt compositions for "normal" MORB, corrected for the effects of olivine fractionation to $8 \mathrm{wt} \% \mathrm{MgO}$, reported by McKenzie and Bickle [1988] and Klein and Langmuir [1987]. Note that both the $M B 88$ and $K L 87$ calculations are too high in $\mathrm{Si}_{8.0}$ and too low in $\mathrm{Fe}_{8.0}$ to account accurately for normal EPR compositions. This results from the fact that both of them calculated equilibrium melting rather than fractional melting (see discussion in text). 


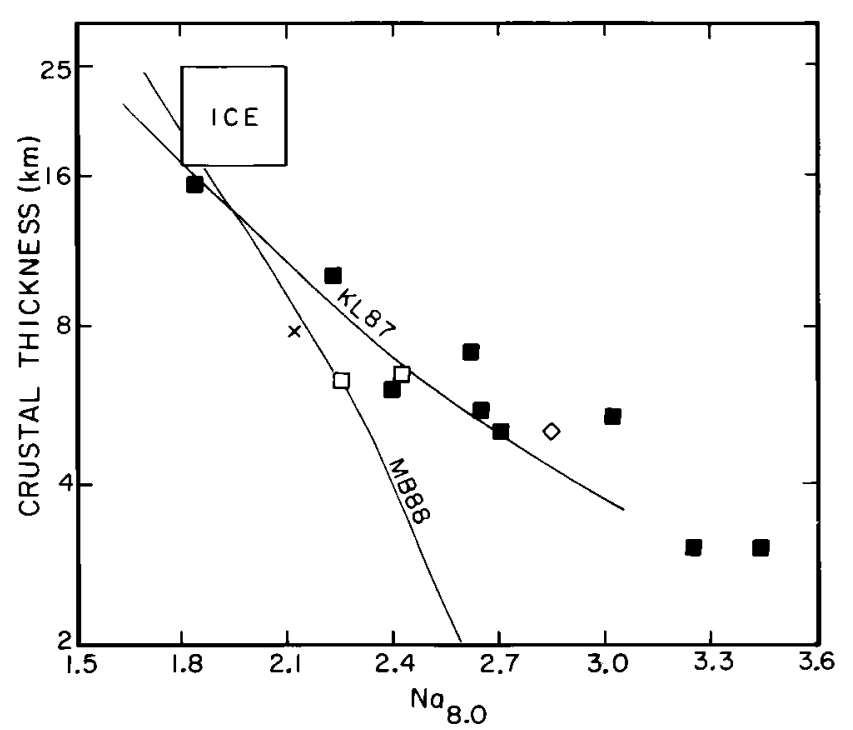

Fig. 44. $\mathrm{Na}_{8.0}$ vs. crustal thickness for observed oceanic crust and calculated pooled melts from $K L 87$ and $M B 88$. The steepness of the $M B 88$ results from the high effective partition coefficient for the $\mathrm{Na}_{2} \mathrm{O}$ calculations in their model (see Figure 42). C.rustal thickness data from Figure 18.

parameterization produces melts that have too little change in $\mathrm{FeO}$, and generally too low $\mathrm{FeO}$ and too high $\mathrm{SiO}_{2}$ contents (Figure 43).

In spite of our criticisms of the $M B 88$ chemistry function, we should not lose sight of the fundamental agreement between the conclusions of $K L 87$ and $M B 88$. Both studies suggest that partial melting of the mantle exerts a major control on MORB chemistry, and particularly on $\mathrm{Na}_{2} \mathrm{O}$ contents. Both suggest that the differences in partial melting are in response to mantle temperature, and correlate with crustal thickness. Both use equilibrium melting and a linear mixing function to calculate mean melt compositions. All of these factors indicate substantial agreement on the big issues concerning melt formation and migration. The agreement between the two studies also extends to an important discrepancy between the $\mathrm{Fe}_{8.0}$ and $\mathrm{Si}_{8.0}$ calculated from peridotite melting experiments and those observed in MORB.

Niu and Batiza [1991] used a different approach in the formulation of their chemistry function based on the empirical derivation of apparent D's for each oxide as a function of $P$ and $F$ for different bulk compositions. One of their goals was to improve upon the approach of $K L 87$ and $M B 88$ by modeling the effects of fractional, rather than equilibrium, melting. Their mixing function involves the pooling of melt compositions produced at $1 \%$ melt increments for different pressures of intersection of the solidus and final pressures of melting. Their melting function is based on an adiabatic gradient during melting of $6^{\circ} / \mathrm{kbar}$ from McKenzie [1984] and melting contours from the experiments of Jaques and Green [1980].

The $N B 91$ approach reproduces the experiments of Jaques and Green more reliably than $M B 88$, and captures the behavior of
$\mathrm{Na}$ and $\mathrm{Ca}$ well. Thus the chemistry function for equilibrium melting is useful. There are difficulties with the application of the chemistry function to the general melting problem, however. First, it is not clear whether an apparent D determined for equilibrium melting at relatively large extents of melting can be used to model fractional melting. Second, for their melting function $N B 91$ superimpose a melting adiabat from McKenzie [1984] onto melting contours determined by Jaques and Green [1980]. This juxtaposition does not take into account the thermodynamic link between temperature path and extent of melting, and causes their melting function to produce a large extent of melting near the solidus, without the requisite temperature drop that is required to supply the heat of fusion. This leads to an impossibly high value to $5 \%$ melt per kbar of pressure release near the solidus. Finally, $N B 91$ do not use a suitable mixing function that is tied to physically realistic models of steady-state melt generation. Therefore although $N B 91$ provide a useful parameterization of equilibrium melting experiments, their detailed conclusions concerning MORB generation need to be reconsidered in light of the need for a combination of appropriate chemistry, melting and mixing functions to model mantle melting beneath ridges.

\section{New QuanttTative Model for Mantle Melting}

There are drawbacks to all three melting models discussed above. First, because equilibrium melting is ideally the type of melting that occurs in the experiments, any method based on empirical reproduction of the experimental results can be reliable only for equilibrium melting. Answering the question of how the mantle melts depends critically on being able to quantitatively model a variety of melting processes in addition to equilibrium melting, such as fractional melting and continuous melting. Second, previous model results are inflexible with respect to mantle source composition, in that they cannot reliably model mantle compositions that differ significantly from those of the experimental starting compositions. Thus, these models cannot be used to address questions of mantle heterogeneity.

These two difficulties can be overcome by an alternative approach to calculating the chemistry function for mantle melting. If partition coefficients between individual minerals and liquid can be parameterized as a function of temperature, pressure and liquid composition, then equilibria can be calculated rigorously. This approach has been used effectively for the calculation of fractional and equilibrium crystallization [Langmuir and Hanson, 1981; Bender et al., 1984; Nielsen and Dungan, 1983; Weaver and Langmuir, 1990], for the evaluation of complex crystallization processes [e.g. Nielsen, 1989, 1990; Langmuir, 1989], and is the method used to evaluate high pressure crystallization in this paper for the results of section 6.3 and Appendix C. The equations apply equally to melting and crystallization, and can be applied to a diversity of compositions and processes.

The $\mathrm{K}_{\mathrm{d}}$ approach to calculating chemical equilibria, like the other chemistry functions discussed in section 4.4, depends on an experimental data base. However, it has the advantage that 
the necessary data can be obtained from a variety of experiments, even those in which only one solid phase is present and the melt fraction is large. This contrasts with the approach that monitors change in the liquid composition and requires saturation of the liquid with multiple solid phases [e.g., Stolper, 1980; Fujii and Scarfe, 1985; Kinzler and Grove, $1992 a$ ]. Thus the $K_{d}$ approach allows the use of experiments that have the fewest experimental difficulties and is able to make use of a much larger experimental data base.

The $K_{d}$ approach we use is not similar to the approach of $N B 91$, although they also use "distribution coefficients" for their calculations. The distribution coefficients used by NB91 are bulk distribution coefficients (D) for a multiphase assemblage. While $\mathrm{K}_{\mathrm{d}}$ 's can be parameterized as a function of $T, P$, and composition and can be applied to a varicty of conditions, $N B 91$ parameterize their D's as a function of melt fraction, which depends on the bulk composition and the melting process. Their results, therefore, apply rigorously only to a single bulk composition and a single process, equilibrium melting.

For the complete calculation of mantle melting, it would be necessary to consider all components and all possible phases. Then the proportions and compositions of all phases in the system could be determined for a given bulk composition. But such a complete understanding of partition coefficients for the mantle system is lacking. For melting of the mantle, however, there is an a priori constraint that allows $\mathrm{MgO}$ and $\mathrm{FeO}$ to be calculated even in the absence of complete characterization of the partition coefficients for all elements and all phases. The constraint is that olivine is always a residual phase, and hence the liquid must be in equilibrium with olivine at each pressure, temperature and extent of melting required by the melting function. This constraint allows the calculation of the $\mathrm{FeO}$ and $\mathrm{MgO}$ contents of the liquid as melting proceeds. The specific equations that allow this calculation are presented in Appendices $\mathrm{A}$ and $\mathrm{B}$. We emphasize that the calculations require that olivine be present in the residue, but do NOT require that olivine be the only residual phase. The olivine-melt $K_{d}$ 's must be obeyed regardless of what other phases exist. The calculations apply from solidus to liquidus as melting proceeds.

For the accurate calculation of $\mathrm{MgO}$ and $\mathrm{FeO}$ contents during melting, it is necessary that the temperature, pressure and compositional controls on the olivine partition coefficients be taken into account. There have been many investigations of olivine/liquid equilibria, over a substantial range of $P, T$ and $X$, and several hundred experiments are available to calibrate the $\mathrm{K}_{\mathrm{d}}$ 's (see references in Appendix B). We have re-examined these experiments in order to develop appropriate equations for the $\mathrm{FeO}$ and $\mathrm{MgO} \mathrm{K}_{\mathrm{d}}$ 's for the calculations. In addition to $\mathrm{T}$ and $P$, the olivine $K_{d}$ 's are sensitive to melt composition, particularly to the concentration of the alkalis [e.g., Roeder, 1974; Ford et al., 1983; Kinzler and Grove, 1992a; and Appendix B]. For a given $\mathrm{P}$ and $\mathrm{T}$, the olivine $\mathrm{K}_{\mathrm{d}}$ 's increase substantially with increasing alkali content. The parameterization of the $\mathrm{Fe}$ and $\mathrm{Mg} \mathrm{K} \mathrm{K}_{\mathrm{d}}$ 's in olivine is sufficiently precise that it should lead to relatively small errors in the melting calculations. The average error of recovery for the partition coefficients over a much wider range of pressure, temperature and composition than is relevant to mantle melting is $5 \%$, which is on the order of $0.5 \mathrm{wt} \% \mathrm{FeO}$ or $\mathrm{MgO}$ (Appendix B). The compositional dependence of the $\mathrm{Fe}$ and $\mathrm{Mg} \mathrm{K} \mathrm{d}_{\mathrm{d}} \mathrm{s}$ is a major difference between these calculations and those carried out by Langmuir and Hanson [1980] and in the earlier version of this paper distributed in July 1990 . The earlier calculations used only a temperature dependence on the olivine partition coefficients with no compositional dependence.

Partition coefficients also permit the calculation of major elements such as $\mathrm{TiO}_{2}, \mathrm{Na}_{2} \mathrm{O}$ and $\mathrm{K}_{2} \mathrm{O}$ that behave similarly to trace elements. We treat these three elements as trace elements, but for $\mathrm{TiO}_{2}$ and $\mathrm{Na}_{2} \mathrm{O}$ the partition coefficients change with temperature, pressure, and the fraction of clinopyroxene in the residue. As discussed in Appendix B, these two elements seem to have different dependencies on pressure and temperature. The $\mathrm{Na} \mathrm{K}_{\mathrm{d}}$ for clinopyroxene has a particularly strong pressure dependence, presumably due to the increased stability of the jadeite component in clinopyroxene with increasing pressure. The effects of liquid composition on these partition coefficients should be added as more experimental data become available, but in general the values of the $K_{d}$ 's are small enough that compositional variation should not have a large effect on the computed results, once melting proceeds beyond a few percent.

To carry out the calculation, the source composition and mantle temperature at some depth below the solidus must be specified. Pressure and temperature for the initiation of melting are then determined by the mantle solidus (see Figure 31a). The subsequent relationships among temperature $(\mathrm{T})$, pressure $(P)$ and extent of melting $(F)$ are given by the melting function discussed in section 4.2. T, P and F from the melting function are used to calculate the $\mathrm{K}_{\mathrm{d}}$ 's and liquid composition for $\mathrm{Na}_{2} \mathrm{O}, \mathrm{K}_{2} \mathrm{O}$ and $\mathrm{TiO}_{2}$, assuming non-modal melting with clinopyroxene decreasing by $0.7 \%$ in the residue for each percent of liquid produced. This liquid composition, along with $\mathrm{T}$ and $\mathrm{P}$, allows calculation of the $\mathrm{FeO}$ and $\mathrm{MgO} \mathrm{K}$ 's between olivine and liquid. Then the solution to the quadratic equation in Appendix $\mathrm{B}$ gives the $\mathrm{FeO}$ and $\mathrm{MgO}$ contents of liquid and residual solid.

This calculation can be understood intuitively. Because the FeO-MgO exchange distribution coefficient, $\mathrm{K}_{\mathrm{D}}$, is about 0.3 , the $\mathrm{FeO} / \mathrm{MgO}$ of a liquid in equilibrium with any mantle olivine composition is fixed. Thus the liquid composition is constrained on an $\mathrm{MgO}-\mathrm{FeO}$ diagram to a line radiating from the origin. The melting function gives temperature and extent of melting at a given pressure, for a given pressure of intersection of the solidus. The $\mathrm{K}_{\mathrm{d}}$ equations then provide the $\mathrm{K}_{\mathrm{d}}$ 's for $\mathrm{MgO}$ and $\mathrm{FeO}$, and these fix the point of liquid composition on the line. Combining these constraints with the mass balance relationships among melt, source and residue allows the calculation for all extents of melting.

The chemical calculations can be combined with diverse melting and mixing functions to quantify the chemical consequences of mantle melting processes. Specific advantages of the calculations are that they are accurate for the 
elements determined ( $\mathrm{Fe}, \mathrm{Mg}, \mathrm{Na}, \mathrm{K}$ and $\mathrm{Ti}$ ); they permit a quantitative evaluation of different styles of melting, such as fractional or equilibrium melting; they can be used to quantitatively model mantle heterogeneity; and they can be used reliably for the small extents of melting that are difficult to address experimentally. Although the new calculations do not include all the major elements, the five elements considered are calculated rigorously, and independently of the other elements, so the results are valid. Most important for the topics considered here, we are able to calculate the two elements ( $\mathrm{Na}$ and $\mathrm{Fe}$ ) that are particulary diagnostic of MORB variability and thus to evaluate the origin of the local and global systematics of compositional variability.

\subsection{Illustration of Calculated Results}

In the following sections we begin with s.mple examples of melting to illustrate the quantitative results of the new melting model under simple melting conditions.

\subsubsection{Isobaric Melting}

The simplest case to consider is isobaric melting, where melting occurs as the temperature increases at constant pressure. $F$ can be calculated from $\mathrm{dT} / \mathrm{dF}$ (see section 4.2). Knowledge of $\mathrm{T}$ and $\mathrm{F}$ permits calculation of the $\mathrm{Na}_{2} \mathrm{O}, \mathrm{K}_{2} \mathrm{O}$ and $\mathrm{TiO}_{2}$ of the liquid, which permits the calculation of $\mathrm{FeO}$ and $\mathrm{MgO} \mathrm{K} \mathrm{d}_{\mathrm{d}}$ 's, and finally the $\mathrm{FeO}$ and $\mathrm{MgO}$ contents of the liquid. Figure 45 shows results for $\mathrm{MgO}$, and $\mathrm{FeO}$ and $\mathrm{Na}_{2} \mathrm{O}$ for isobaric, equilibrium melting at several pressures. At the lowest extents of melting, the $\mathrm{FeO}$ and $\mathrm{MgO}$ contents are low because the $\mathrm{K}_{\mathrm{d}}$ 's are high due to both high alkali contents in the liquid and low temperatures. $\mathrm{FeO}$ and $\mathrm{MgO}$ both initially increase as the extent of melting increases, though of course the $\mathrm{FeO} / \mathrm{MgO}$ ratio decreases. For a given $\mathrm{MgO}$ content or given extent of melting, $\mathrm{FeO}$ increises with increasing pressure (Figure 45).

\subsubsection{Equilibrium and Fractional Melting during Adiabatic Decompression from $20 \mathrm{kbar}$}

In order to calculate melting during adiabatic ascent, a melting function must specify the amount of melting produced for a given pressure drop (section 4.2). The melting function differs for equilibrium and fractional melting, with fractional melting producing a smaller increment of melting for the same pressure decrease. Because the partition coefficients change with each pressure and temperature, all elements for fractional melting are calculated incrementally, with the liquid entirely removed at each $1 \mathrm{kbar}$ pressure step.

We first present results that do not involve a mixing function. We simply follow the evolution of liquid compositions in a single unit of peridotite as it intersects its solidus and decompresses (Figure 46a). We call these trajectories "unit paths" since they apply to one unit of mantle as it passes through the melting regime. Note that this is distinct from the integrated melts that result from mixing of melts from many unit paths in a melting regime.

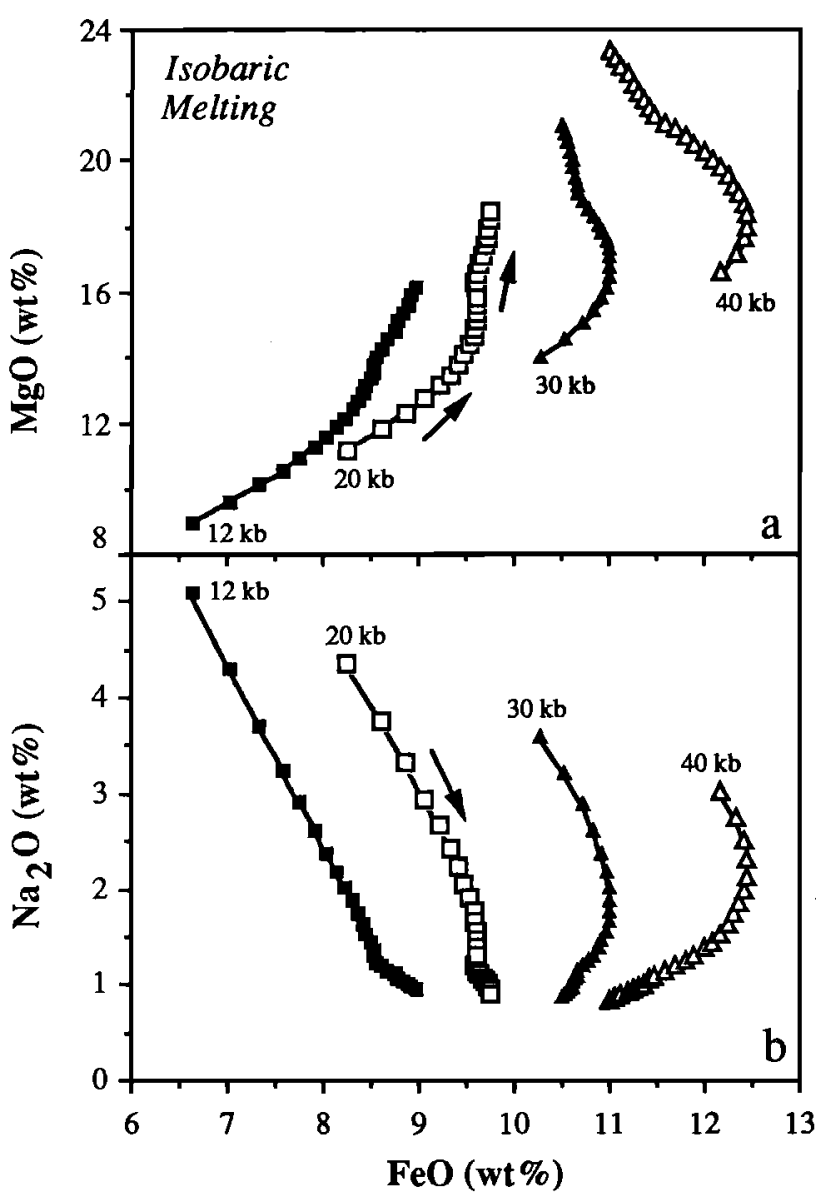

Fig. 45. Results of the new melting model for isobaric melting of the mantle. FeO vs. a) $\mathrm{MgO}$ and $b$ ) $\mathrm{Na}_{2} \mathrm{O}$ for melting at $12,20,30$, and 40 kbar. Arrows indicate increasing degrees of partial melting, from about $2 \%$ to $35 \%$ melting for each curve. The kinks in the paths correspond to where clinopyroxene is no longer a residual phase (at $\sim 22 \%$ melting).

For equilibrium melting, the liquid stays with the residue as melting proceeds, and the curve in Figure $46 a$ shows the progressive evolution of the liquid at the different pressures within the melting regime. For fractional melting, liquid is extracted at each pressure step. Figure $46 a$ shows both the instantaneous melts produced at each pressure step, and the progressive change of the accumulated fractional melts, where each melt produced along the unit path is mixed with those produced previously. In all cases, the $\mathrm{FeO}$ contents first increase at low extents of melting, then decrease as melting proceeds. The early increase is due to the compositional effects on the partition coefficients. The $\mathrm{Na}_{2} \mathrm{O}$ and $\mathrm{K}_{2} \mathrm{O}$ concentrations are high in the first increments of melting, causing the $\mathrm{K}_{\mathrm{d}}$ 's to be relatively high, and $\mathrm{FeO}$ and $\mathrm{MgO}$ to be relatively low (see Figure $46 a$ ). As the extent of melting increases, $\mathrm{Na}_{2} \mathrm{O}$ and $\mathrm{K}_{2} \mathrm{O}$ decrease, causing a decrease in the $\mathrm{K}_{d}$ 's and a corresponding increase in the $\mathrm{FeO}$ and $\mathrm{MgO}$ contents of the liquid. At still higher extents of melting, the alkali contents change relatively little, and the pressure and temperature effects 

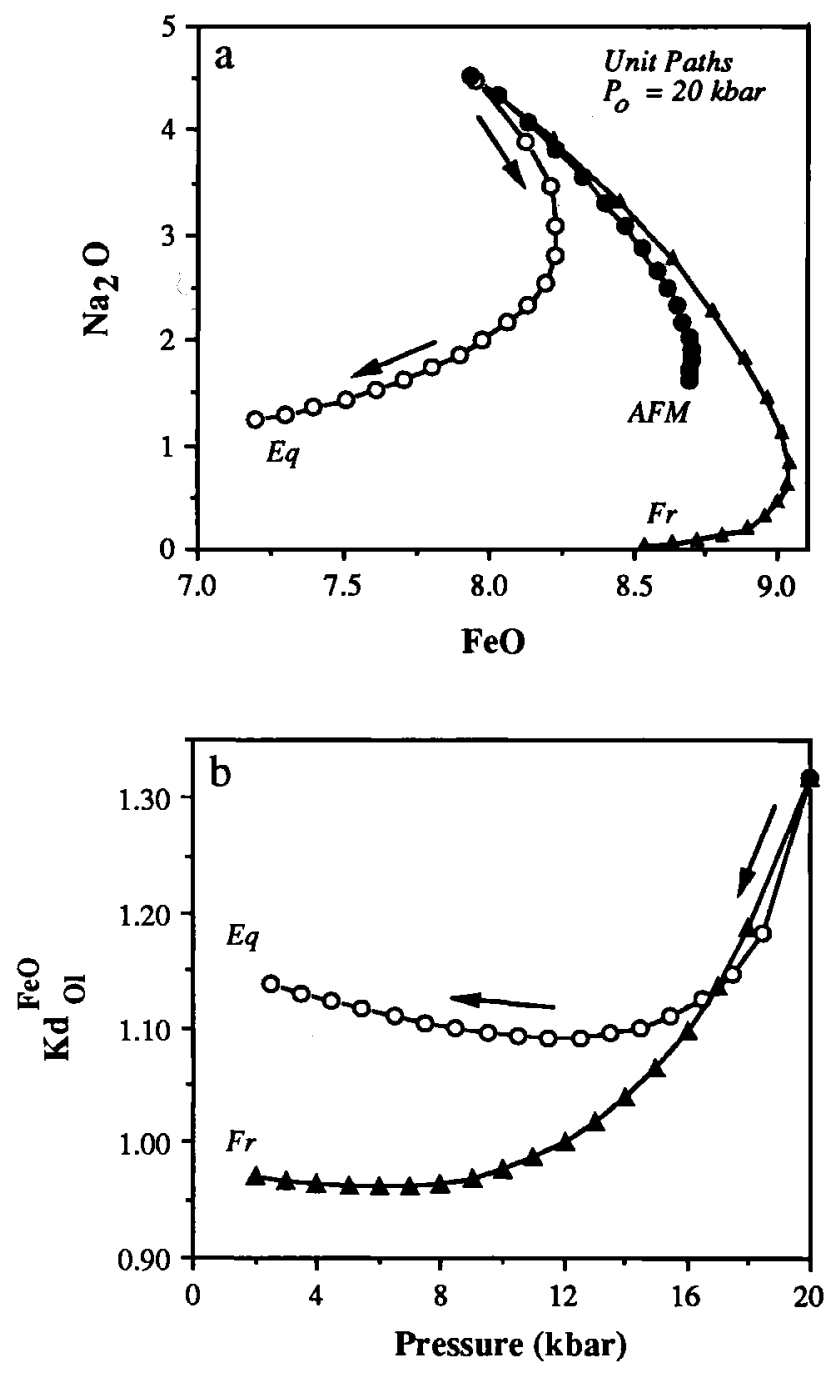

Fig. 46. a) $\mathrm{Na}_{2} \mathrm{O}$ vs. $\mathrm{FeO}$ for melt compositions predicted from the new melting model for a single unit of mantle during progressive adiabatic melting. Three such "unit paths" are shown for equilibrium melting (Eq), fractional melting (Fr), and accumulated fractional melting (AFM) for mantle that intersects the solidus at $20 \mathrm{kbar}$, and melts to $\sim 2$ kbar (a pressure approximately equivalent to the base of the normal ocean crust). The AFM path results from summing the melts along the fractional unit path. Arrows show direction of increasing extent of melting (melting paths begin at $2 \%$ melting). Each point along the curve represents a 1 kbat drop in pressure. b) Variation in the olivine/melt $\mathrm{K}_{\mathrm{d}}$ for $\mathrm{FeO}$ as a function of pressure along the unit path for equilibrium and fractional melting. $K_{d}$ is calculated as described in Appendices A and B and section 5.0.

dominate. Since the $\mathrm{FeO}$ concentration in the melt is sensitive to pressure, FeO decreases substantially along the lower pressure portions of the unit paths.

The contrasts between the fractional and equilibrium unit paths can be understood as a consequence of the different temperatures and liquid compositions that exist at a given pressure. Fractional melting strips most of the alkalis from the residue at low extents of melting. This causes large decreases in the $K_{d}$ 's at low extents of melting and more rapid increase of
$\mathrm{FeO}$ in the liquid (Figures $46 a$ and $46 b$ ). The temperature of the peridotite for fractional melting is also higher at any given pressure and extent of melting. This causes the $\mathrm{K}_{d}$ 's to be lower, and the $\mathrm{FeO}$ contents higher. The combination of these effects causes the fractional melts to be substantially richer in $\mathrm{FeO}$ than the equilibrium melts.

Kinzler and Grove ( $1992 a$ and $b$ ) have recently and independently carried out calculations of mantle melting based on a different experimental data set and an entirely different method of calculation. They also found curved melting paths due primarily to compositional effects on olivine partitioning. similar to those presented here.

\subsection{Importance of the Melting Process to Calculated Liquid Compositions}

In Figure 46a, the three different melting processes lead to distinct paths of liquid compositions, which illustrates the importance of understanding the physical aspects of the melting process in evaluating models of basalt pelrogenesis from experimental data. Contrasts become even more pronounced when diverse mixing functions are included. It is clear, therefore, that interpretation of MORB data cannot be made solely from equilibrium experiments alone, but depends critically on the melting process. Although this complicates the straightforward interpretation of experimental data, it is nonetheless advantageous. Because different melting processes lead to different effects on melt compositions, basalt compositions can be used to evaluate the melting processes that gave rise to them.

\subsection{Calculation of Equilibrium and Fractional Melting Beneath Ocean Ridges}

To apply the calculations to ocean ridges, we need to use appropriate mixing functions. As discussed in section 4.3, these functions differ for equilibrium and fractional melting. For equilibrium melting, we can simply sum the melts as indicated in the RMC. For fractional melting, each "box" of the RMC has provided fractional melts from the total range of pressures along the unit path, so there is an additional step of integration that is required to sum the instantaneous melts into an accumulated fractional melt. Therefore calculation of fractional melting requires summing of the melts along the unit path, to calculate each box of the RMC, and then suniming the resulting AFM liquid from each box. Figures 47 and 48 illustrate both the unit paths within the melting regime and the integrated mean melts from the entire melting regime.

Calculated melt compositions for equilibrium and accumulated fractional melting are shown in Figures 47 and 48 for various pressures of intersection of the solidus $\left(\mathrm{P}_{\mathrm{o}}\right)$. As $\mathrm{P}_{0}$ increases, so docs the overall extent of melting, and the resulting mean melts have lower $\mathrm{Na}$ and higher Fe. Fractional melting leads to higher $\mathrm{Fe}$ than equilibrium melting for a given $\mathrm{Na}$, due to a combination of four factors. (1) Above, we discussed the effects of fractional melting on the $\mathrm{K}_{d}$ 's, which leads to higher Fe for the same unit path (Figures $46 a$ and $46 b$ ). 


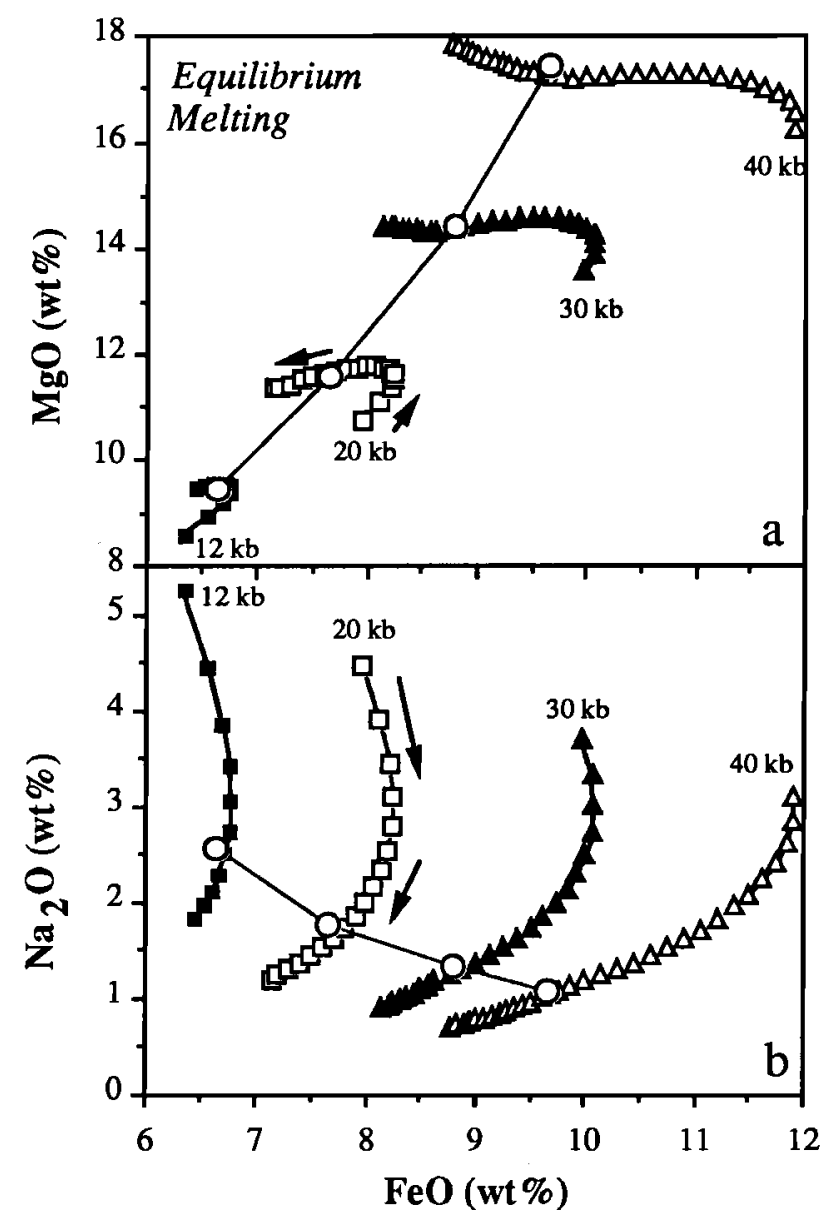

Fig. 47. FeO vs. a) $\mathrm{MgO}$ and b) $\mathrm{Na}_{2} \mathrm{O}$ for equ ilibrium melts of the mantle, calculated from the new melting model. Each curve represents the family of melt compositions produced within the melting regime for a given pressure of intersection of the solidus (as labeled). Arrows show direction of increasing extent of melting (melting paths begin at $2 \%$ melting). Each point along the curve represents a 1 kbar drop in pressure. Open circles are melt compositions that result from pooling the melts along each curve.

(2) Pooling melts from throughout the melting regime leads to further increases in $\mathrm{Fe}$. The mean pressure for any given column in the melting regime is twice as high for fractional melting (Figure 34 and section 4.3.2), leading to higher $\mathrm{Fe}$ melts. (3) The range of pressures for the various accumulated fractional melts from a single melting regime is lower because the central portion of the melting regime has a mean pressure of about half $\mathrm{P}_{\mathrm{o}}$, rather than a pressure corresponding to the base of the crust. The more limited range in pressures leads to a smaller decrease in $\mathrm{FeO}$ contents with greater extents of melting within any given melting regime. (4) Finally, the mean extent of melting is slightly lower, leading to slightly higher $\mathrm{Na}$ in the mean melts for any $\mathrm{P}_{\mathrm{o}}$.

One other important difference between equilibrium and fractional melting is the range in $\mathrm{FeO}$ for mean melts produced by a range in $P_{0}$. From $P_{\circ}$ of 12 to $40 \mathrm{kbar}, \mathrm{FeO}$ varies from 7 . $11.5 \mathrm{wt} \%$ for fractional melting regimes, but only $6.5-9.5 \mathrm{wt} \%$ for equilibrium melting regimes (Figures 47 and 48). The range in mean pressure as a function of $P_{o}$ is greater for fractional melting, and this leads to the greater range in $\mathrm{FeO}$ contents. For the evaluation of mean melts, therefore, fractional melting regimes produce higher overall $\mathrm{Fe}$ contents and a greater range in $\mathrm{FeO}$ contents.

It is important to consider the effects of uncertainties on the calculated results. Figure 49 shows the effects of changing various parameters in the melting function on melt compositions. The solid black line is the standard model we have been using, with a solidus slope and intercept of $13 \% \mathrm{kbar}$ and $1150^{\circ} \mathrm{C}$, and a heat of fusion of $180 \mathrm{cal} / \mathrm{gm}$. Other curves show the effects of a $1^{\circ}$ change in the solidus slope (which has an uncertainty of about $1^{\circ}$ ), the effect of changing the zero intercept of the solidus to $1100^{\circ}$ from $1150^{\circ} \mathrm{C}$, and the effect of changing the latent heat of fusion to $100 \mathrm{cal} / \mathrm{gm}$ from 180 $\mathrm{cal} / \mathrm{gm}$. The change in solidus intercept has the largest effect, causing a difference of about $1 \mathrm{wt} \% \mathrm{FeO}$. These uncertainties affect the absolute value of $\mathrm{FeO}$, but not the relative changes in $\mathrm{FeO}$ for different pressures of intersection of the solidus. For example, in Figure 47 the effect of a charge in the temperature

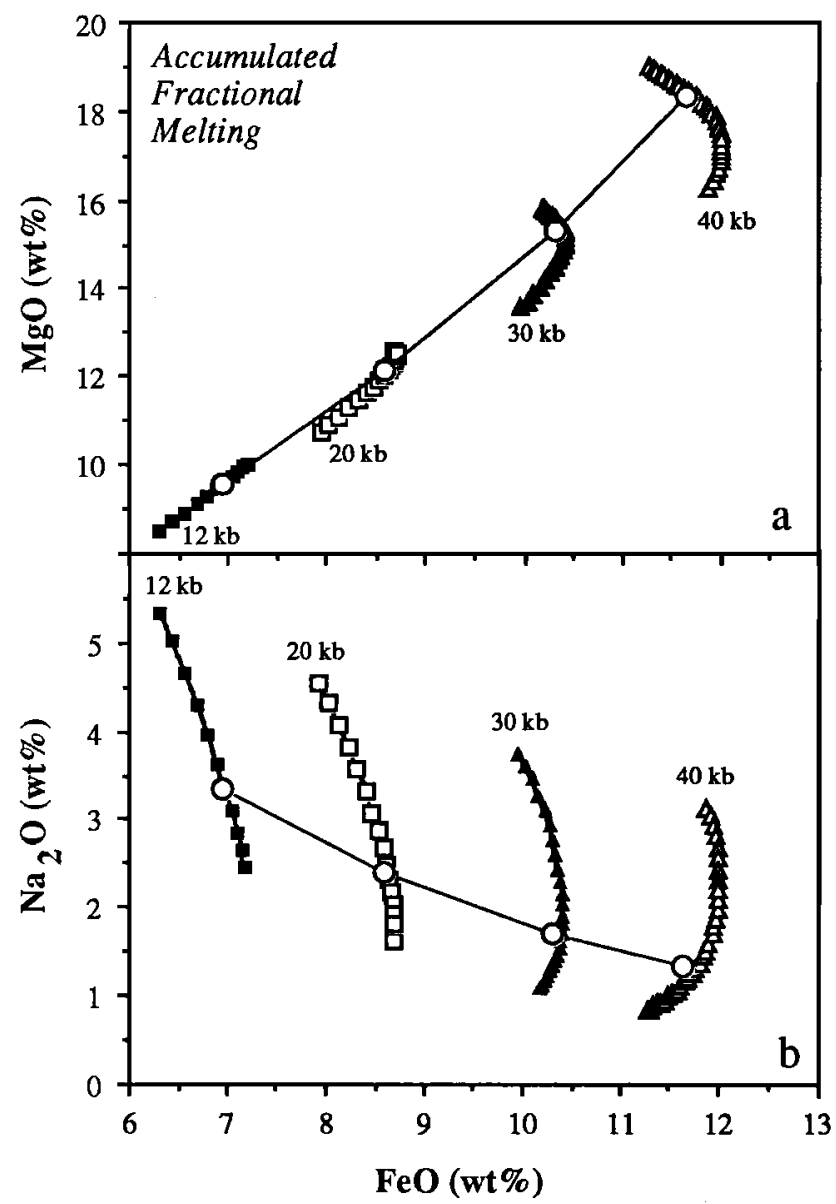

Fig. 48. As Figure 47, for accumulated fractional melting. 


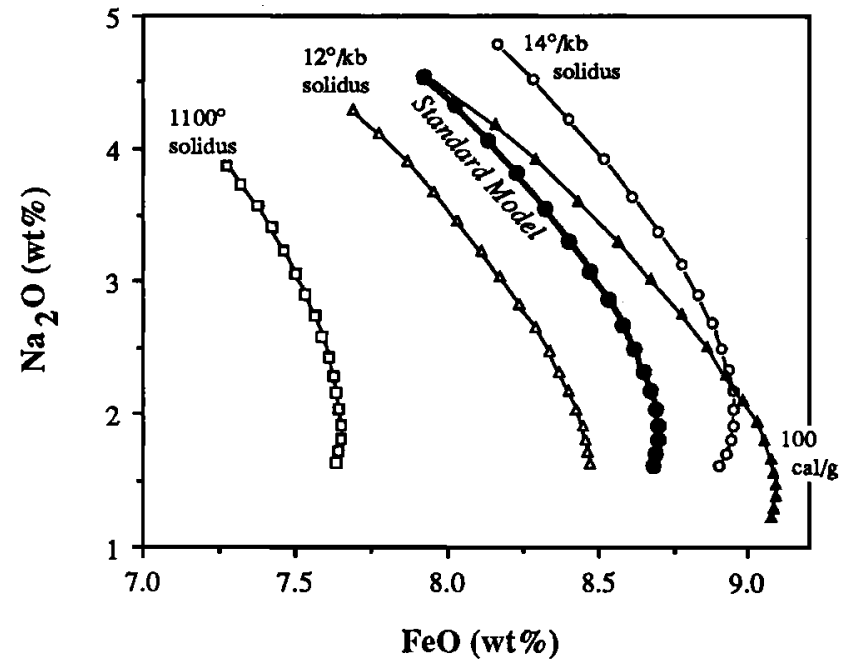

Fig. 49. $\mathrm{FeO}$ and $\mathrm{Na}_{2} \mathrm{O}$ concentrations of calculated mantle melts for different melting parameters for a pressure of intersection of the solidus of $20 \mathrm{kbar}$. The "standard model" in this figure is for accumulated fractional melting, not equilibrium melting. Each curve shows the accumulated fractional melt composition. A lower temperature intercept for the solidus $\left(1100^{\circ} \mathrm{C}\right)$ causes all $\mathrm{K}_{\mathrm{d}}$ 's to be higher, and hence $\mathrm{FeO}$ and $\mathrm{Na}_{2} \mathrm{O}$ in calculated melts to be lower. A shallower solidus slope (e.g. $12^{\circ} \mathrm{kbar}$ ) causes all temperatures to be lower, producing a similar effect. A smaller heat of fusion allows more melting to occur, ultimately leading to lower $\mathrm{Na}_{2} \mathrm{O}$ and higher $\mathrm{FeO}$ contents.

or slope of the mantle solidus would be to move all the curves to higher or lower $\mathrm{FeO}$ contents.

Uncertainties in $\mathrm{Na}_{2} \mathrm{O}$ concentrations are less complicated because the $\mathrm{Na} \mathrm{K}_{d}$ is much less sensitive to temperature and pressure than the $\mathrm{K}_{\mathrm{d}}$ 's for $\mathrm{FeO}$ and $\mathrm{MgO}$ in olivine. The principal uncertainty has to do with the source concentration of $\mathrm{Na}_{2} \mathrm{O}$. A primitive mantle value is $0.30+0.03 \mathrm{wt} \%$ [KL87, Jagoutz et al, 1979], but the mantle that gives rise to MORB may be slightly depleted in $\mathrm{Na}_{2} \mathrm{O}$. Furthermore, Hofmann [1988] has shown that the depletion in the MORB mantle is complementary to the enrichment in continents for a large number of elements. If the continents are subtracted from primitive mantle to form the depleted (MORB) mantle, then this depleted mantle would have a $\mathrm{Na}_{2} \mathrm{O}$ concentration of around $0.26 \mathrm{wt} \%$, or about $10 \%$ depletion relative to primitive mantle. A change in the source concentration of $\mathrm{Na}_{2} \mathrm{O}$ translates dircclly to changes in the $\mathrm{Na}_{2} \mathrm{O}$ concentrations in calculated melts, hence the various melting curves could move $10 \%$ relative in terms of $\mathrm{Na}_{2} \mathrm{O}$ abundances as a result of permissible variations in source concentrations.

\section{Applications to Morb Data}

We now have in place the systematics of the MORB data set and the quantitative tools to evaluate the effects of various melting styles, mixing functions, and mantle source compositions. In order to avoid an exhaustive evaluation of the chemical consequences of all possible variables, we begin by considering the qualitative effects of various models for melt formation beneath ridges. These qualitative considerations permit a rapid evaluation of many variables and enable us to select the most promising ones for a full quantitative treatment.

\subsection{Mean Properties of the Ocean Crust Expected for Different Melting Regimes}

The models discussed in the mixing function section (section 4.3) considered simple melting regimes for a single mantle potential temperature where melt extraction was complete, upwelling was passive, and there was no downward component of mantle flow near the ridge. We will call this the "standard model." In this section, we consider more complex models, such as active flow, incomplete melt focusing, and variations in mantle temperature and their effects on the mean properties of the oceanic crust that is produced. These results can then be used to select appropriate models that are likely to account for the observed data from the ocean crust. We first examine each of the variables in isolation. In Figure 50, each model is drawn to scale, with $1 \mathrm{kbar}$ equivalent to $3 \mathrm{~km}$ of mantle or 3.3 $\mathrm{km}$ of crust and $\gamma$ equal to $1.2 \% / \mathrm{kbar}$ (a good approximation to equations 19 and 20), unless specified otherwise. For each melting regime, the maximum $F\left(F_{\max }\right)$, mean $F(\bar{F})$, crustal thickness $\left(h_{c}\right)$ and mean $P(\overline{\mathbf{P}})$ are calculated, as explained in section 4.3, and these parameters are plotted together in Figure 51. The parameters for the standard model have been chosen such that the resulting pooled melt produces average ocean crust $\left(h_{c}=5.5 \mathrm{~km}, \overline{\mathrm{F}}=10 \%\right)$. It thus serves as a useful reference in discussing the results of the more complex models.

Mantle Temperature. Figure 2 showec: two melting regimes that might result from upwelling mantle of different temperatures. Hot mantle intersects the solidus deeper than cold mantle, so $P_{o}$ and mantle $T$ correlate with one another. Because the melt fraction increases linearly with $P$ for both melting regimes, the equations for line ir mixing that were outlined above (section 4.2) can be used to calculate $\bar{F}(1 / 2$ $\left.F_{\max }\right)$ and $\bar{P}\left(P_{0} / 3+P_{f}^{*} 2 / 3\right)$. Crustal thickness then equals the product of $\overline{\mathrm{F}}$ and the height of the RMC $\left[\overline{\mathrm{F}} *\left(\mathrm{P}_{\mathrm{f}}-\mathrm{P}_{\mathrm{o}}\right)\right]$. Cold mantle intersects the solidus shallower, melts to a lower extent at shallower pressures, and creates less crust than normal mantle (Figures 50a-50b). Crustal thickness increases exponentially with $\overline{\mathrm{F}}$ because both $\overline{\mathrm{F}}$ and the height of the RMC increase with increasing mantle temperature (i.e., $h_{c}=\bar{F} * \bar{F} *$ $2 / 0.012$, see Figure 51a). $\overline{\mathrm{P}}$ and $\overline{\mathrm{F}}$ are positively correlated because a greater amount of melting requires a larger pressure interval of melting, and thus higher mian pressures (Figure $51 b$ ).

Fractional Melting. Melting contours in the melting regime (and RMC) are more widely spaced for fractional melting because more decompression is required to, compensate for the rise of the solidus due to melt loss. To account for this effect, we have used a lower value of $\gamma(1.05 \% / \mathrm{kbar}$, a good approximation to equations (19) and (20) with the recommended changes suggested in section 4.2 for $\mathrm{dT} / \mathrm{dF}$ ). Because of this lower value of $\gamma$, fractional melting leads to a lower maximum and mean extent of melting and thus thinner 
a. Standard

$\left[F \max =20 \%, \overline{\mathrm{F}}=10 \%, \mathrm{~h}_{\mathrm{c}}=5.5 \mathrm{~km}, \overline{\mathrm{P}}=7.2 \mathrm{~kb}\right]$

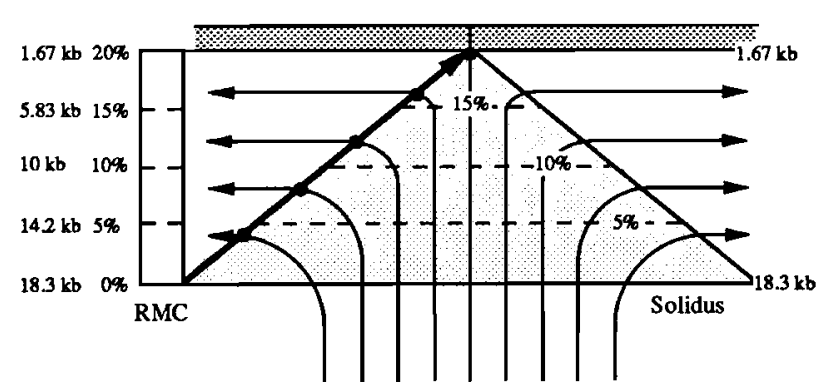

c. Fractional

$\left[F \max =17.6 \%, \quad \bar{F}=8.8 \%, \quad h_{c}=4.9 \mathrm{~km}, \quad \bar{P}=12.7 \mathrm{~kb}\right]$

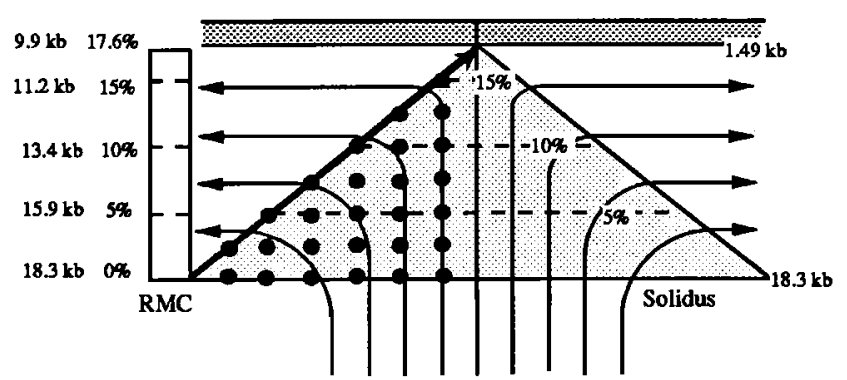

e. Active

$\left[\mathrm{Fmax}=20.0 \%, \quad \overline{\mathrm{F}}=20 \%, \mathrm{~h}_{\mathrm{c}}=11 \mathrm{~km}, \quad \overline{\mathrm{P}}=3.3 \mathrm{~kb}\right]$

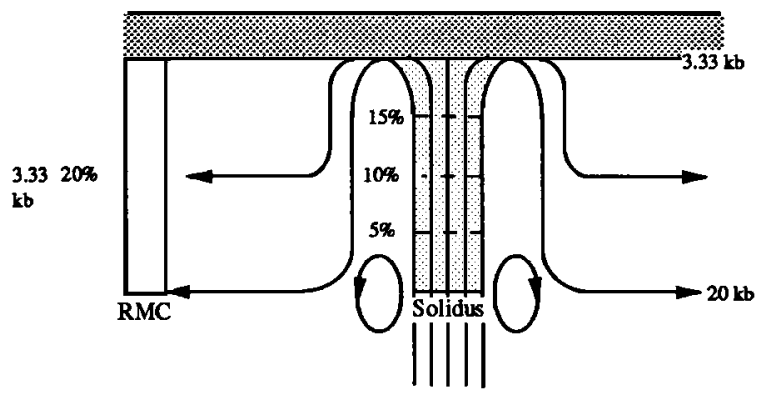

b. Cold Mantle

km $\quad P(\mathbf{k b})$

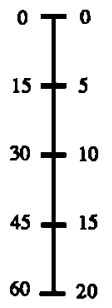

$\left[\mathrm{Fmax}=15.0 \%, \overline{\mathrm{F}}=7.5 \%, \quad \mathrm{i}_{\mathrm{c}}=2.1 \mathrm{~km}, \quad \overline{\mathrm{P}}=5.1 \mathrm{~kb}\right]$

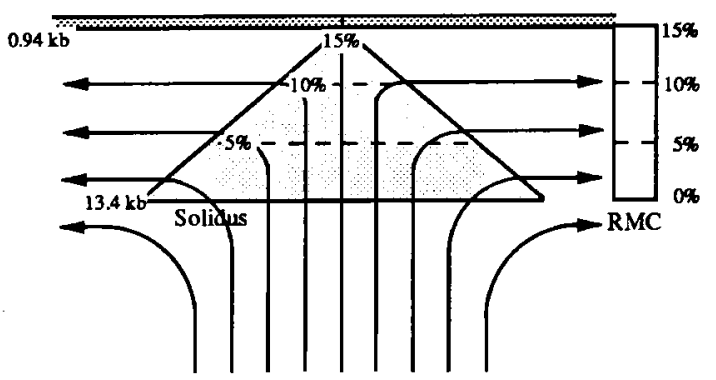

d.Thick Lithosphere

$\left[F \max =11.0 \%, \quad \bar{F}=7.8 \%, \quad h_{c}=4.0 \mathrm{~km}, \quad \bar{P}=10.0 \mathrm{~kb}\right]$

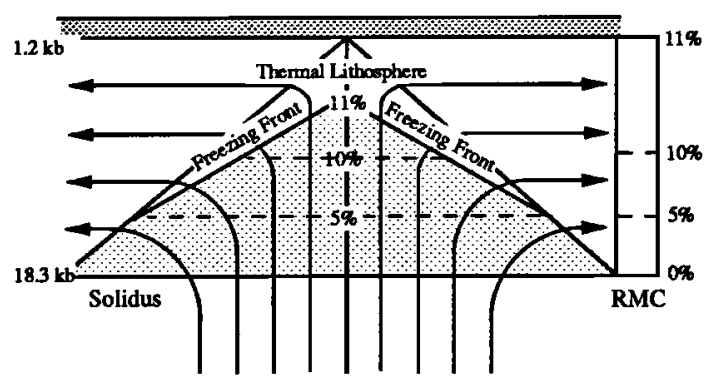

f. Incomplete Focusing

$P(\mathbf{k b}) \quad \mathbf{k m}$

$\left.{ }_{10}^{0}\right]_{30}^{0}$ $\mathbf{k m} \quad \mathbf{P}(\mathbf{k b})$

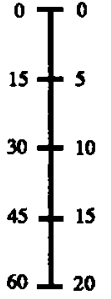

Fig. 50. Different models for the melting regimes. All melting regimes are drawn to scale, where $1 \mathrm{kbar}$ is equal to $3 \mathrm{~km}$ of mantle or $3.3 \mathrm{~km}$ of crust, and $\gamma$ is $1.2 \% / \mathrm{kbar}$, unless noted otherwise. The mean properties of the steady-state ocean crust generated by each melting regime are given, and may be calculated from the RMC. $\overline{\mathrm{F}}$ is the mean extent of melting, $\overline{\mathrm{P}}$ is the mean pressure of melting, $h_{c}$ is crustal thickness, and $F_{\max }$ is the maximum amount of melting in the melting regime. a) The standard model is for equilibrium melting and compete melt focusing. This standard model generates average oceanic crust (e.g., $\mathrm{h}_{\mathrm{c}}$ of $5.5 \mathrm{~km}$, and $\overline{\mathrm{F}}$ of $10 \%$ ). b) Cold mantle intersects the solidus shallower leading to lower $\overline{\mathrm{F}}, \overline{\mathrm{P}}$ and $\mathrm{h}_{\mathrm{c}} . c$ ) Fractional melting is modeled here with a lower $\gamma(1.05 \% / \mathrm{kbar})$ and continuous melt extraction. Crust produced by fractional melting is slightly thinner and has lower $\overline{\mathrm{F}}$ than the standard model, and $\overline{\mathrm{P}}$ is much higher. $d$ ) The melting regime beneath thick lithosphere is compressed by a freezing front. The melting contours are more widely spaced, because mantle may no longer upwell adiabatically. The resulting oceanic crust has lower $\vec{F}$ and higher $\vec{P}$ than the standard model. e) Active flow is exaggerated here, where all mantle melts to the base of the crust. Active flow leads to thicker crust, higher $\overline{\mathrm{F}}$ and lower $\overline{\mathrm{P}}$ than the standard model. $f$ ) If melting the "wings" of the melting regime is not focused into the ridge, crust formed at the ridge will be thinner, but derived by higher $F$ and lower $\bar{P}$ than the standard model because the high $P$ and low F melts in the comers of the melting do not contribute to the melt erupted at the ridge axis.

crust than the standard model for the same $\mathrm{P}_{\mathrm{o}}$ (Figures $50 a-50 \mathrm{c}$ ). In practice, however, it would be difficult to distinguish on the basis of $\bar{F}$ and $h_{c}$ alone between fractional melting and a slightly cooler mantle (see Figure 51a).
A major difference between fractional and equilibrium models, however, is the mean pressure of melting. For the same mantle temperature $\left(P_{0} \sim 18 \mathrm{kbar}\right)$, fractional melting leads to higher mean pressures of melting $(\bar{P}=12.7 \mathrm{kbar})$ than 

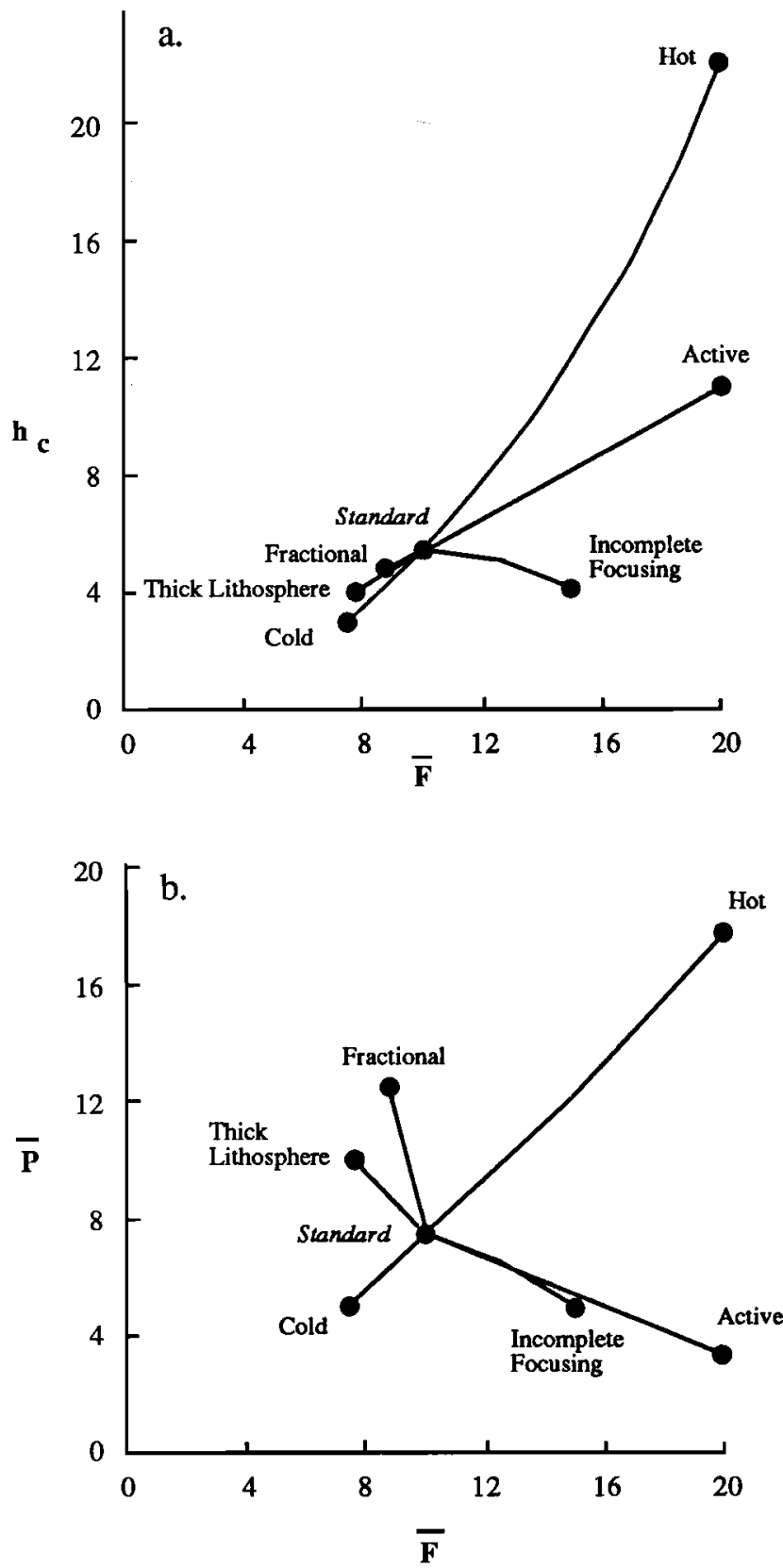

Fig. 51. Properties of the oceanic crust generated by the six melting regime models in Figure 50; mean extent of melting $(\overline{\mathrm{F}})$; mean pressure of melting $(\bar{P})$; crustal thickness $\left(h_{c}\right)$. This figure can be used to infer the progressive variations that would be produced by each process identified in the figure. For example, a small component of active upwelling would slightly lower $\bar{P}$ and raise $\bar{F}$. A combination of active upwelling and fractional melting would cause little change from the equilibrium melting standard model.

equilibrium melting ( $\overline{\mathrm{P}}=7.2 \mathrm{kbar}$ ) (Figures $50 a-50 \mathrm{c}$ ). This arises because the mean pressure for an equilibrium melt is the minimum pressure for any vertical column in the melting regime, while the mean pressure for an accumulated fractional melt is half the pressure interval of that column (Figures 34a-
$34 b$ and Figures 50a-50c). Pooled fractional melts are thus distinct from pooled equilibrium melts in that they will reflect higher $\overline{\mathrm{P}}$ for similar mean extents of melting (Figure $51 \mathrm{~b}$ ).

Thick Lithosphere. Figure 50d illustrates the possible effects on the melting regime of a thick thermal lithosphere, which may cause melting to stop at depths greater than the base of the crust. Such a situation might correspond to the melting regime adjacent to a fracture zone, or to a very slow-spreading ridge. The slowly upwelling mantle may no longer follow an adiabat and even cease to melt during upwelling at shallow levels. The upper boundary of the melting regime in this case coincides with a freezing front due to the deep penetration of the conductive thermal boundary layer. The melting regime does not extend to the base of the crust (as it does in the other models considered here). For the same mantle temperature $\left(\mathrm{P}_{\mathrm{o}}\right.$ $18 \mathrm{kbar}$ ) and melting process (equilibrium), the thick conductive boundary layer leads to lower $F_{\max }$, lower $\overline{\mathrm{F}}$, higher $\overline{\mathrm{P}}$ and thinner crust (Figure 50d). These effects are similar to those produced by fractional melting, but the effects on $\overline{\mathrm{F}}$ and crustal thickness are greater for thick lithosphere.

Active Flow. Extra buoyancy due tc melt retention or a decrease in mantle density upon melt depletion may lead to extra buoyancy and active upwelling beneath ridges [e.g., Scott and Stevenson, 1989]. Active upwelling may involve a narrowing of the zone of upwelling, more rapid rates of upwelling, and downward flow outside of the melting regime. These effects are exaggerated in Figure 50e, where flow is completely active. All mantle flows to the base of the crust, then "spills downward" to fill the RMC, In this end-member case, all the mantle melts to the maximum amount $\left(\overline{\mathrm{F}}=\mathrm{F}_{\max }\right)$ at the shallowest pressure $\left(\overline{\mathrm{P}}=\mathrm{P}_{\mathrm{f}}\right)$. Compared to the standard model, the totally active flow doubles $\bar{F}$ and substantially lowers $\overline{\mathbf{P}}$ (Figure 51a). Crustal thickness is doubled as well due solely to the doubling of $\overline{\mathrm{F}}$, since the height of the RMC is the same in the two cases. Because crustal thickness increases as a linear function of $\mathrm{F}$ in this model, the traiectory in Figure $51 a$ between totally passive and totally active flow is linear, and differs substantially from the exponential relationship generated by mantle T. The relationship between $\bar{P}$ and $\bar{F}$ for totally active flow is of the opposite slope to the mantle $T$ curve because $\overline{\mathrm{P}}$ decreases dramatically as $\overline{\mathrm{F}}$ increases (Figure $51 b)$.

Incomplete Melt Focusing. Models thus far have assumed complete extraction of all melt at the ritge axis. It is likely, however, that some melt in the furthest corners of the melting regime never contributes to on-axis volcanism. Figure $50 \mathrm{f}$ illustrates incomplete focusing, where melt is focused and extracted only from the central shaded region. The RMC in Figure $51 f$ reflects the incomplete focusing; it is truncated at the base and contains only residual mantle that has melted more than $10 \%$. Mantle processed through the melting regime at greater depths than the $10 \%$ contour does not lose melt to the ridge axis and thus is not part of the RMC. The primary consequence of such incomplete focusing is to increase $\bar{F}$ and decrease $\overline{\mathrm{P}}$ (Figure $51 b$ ), because the deep, low degree melts never contribute to ocean crust at the ridge axis. Although the effects on $\bar{F}$ and $\bar{P}$ are similar to active upwelling, much less 
crust is produced by incomplete melt focusing because much of the melt never makes it to the ridge (Figure 51a). Incomplete melt focusing thus leads to greater mean extents of melting and thinner crust compared to the standard model.

\subsubsection{Variability of individual melts produced by different melting regimes}

The discussion above is pertinent to the mean melts produced by different melting regimes and hence is most relevant to the global variability of ocean ridges, where compositions are averaged over substantial lengths of ridge. Local variability, on the other hand, is more likely a response to variations that occur within a single melting regime [KL89]. It is of interest, therefore, to consider how the different melting regimes affect the pressures and extents of melting for individual melt parcels

Conceptually, for equilibrium melting the individual melts are those that are produced at the tops of each vertical column in the melting regime (Figure $34 a$ ). Equilibrium melts are thus formed within the standard melting regime between $\sim 2$ and 18 kbar and 0-20\% melting (Figure 50a). On a P-F diagram, these melts form a trajectory with a slope that corresponds to $\gamma$ of $1.2 \% / \mathrm{kbar}$ (Figure 52). The inverse relationship between $\mathrm{F}$ and $P$ is simply a consequence of pressure release melting: low extents of melting occur deep in the melting regime, while high extents of melting occur closer to the surface. Since decompression melting is the mechanism of melt formation beneath ridges, all models will lead to an inverse relationship between $\mathbf{F}$ and $\mathbf{P}$. Note that this inverse relationship for melts produced within the melting regime for a given mantle temperature (given $P_{0}$ ) is oblique to the trajectory of mean melts produced by different mantle temperatores (different $P_{o}$ 's).

The fact that pressure release melting occurs for all the models means that melts produced beneath a thick thermal lithosphere or by incomplete melt focusing lie on the same melting curve as the standard model (Figure 52). However, the effect of a thicker thermal lithosphere is to restrict melting to the lower part of the melting regime, while the effect of inefficient focusing is to sample only the central, shallower region of the melting regime. Thus, a single mantle temperature can produce quite different families of compositions, depending on the lithospheric thickness and efficiency of focusing.

Melts from a fractional melting regime are potentially more variable, since they are extracted from throughout the melting regime rather than just from the top of each melting column within the melting regime (see Figure $50 c$ ). The accumulated fractional melts generated from individual vertical columns within the melting regime have equilibratid at higher pressures than the equilibrium melts tapped from the tops of these columns, (Figures 34 and 50). Thus, for a given extent of melting the melt trajectory for accumulated fractional melts lies at higher pressures than for equilibrium melts (Figure 52). The different melting function $(\gamma)$ for fractional melting also leads to a different P-F slope (Figure 52).

Similarly, the results for active upwelling depend on whether melt is extracted from throughout the melting regime, or just

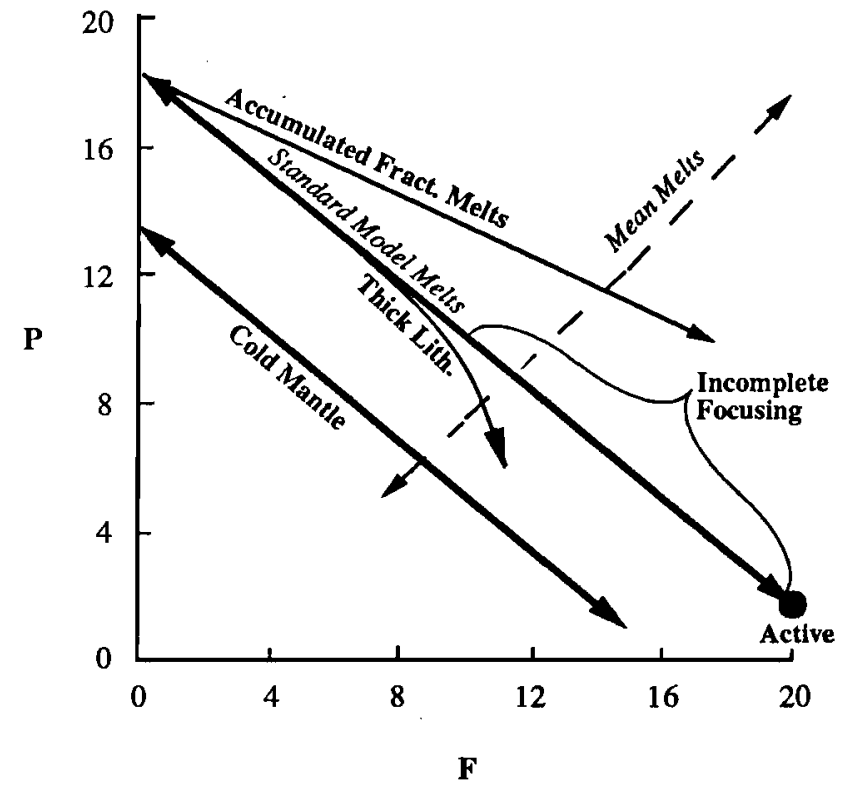

Fig. 52. The properties ( $F$ and $P$ ) of the individual melts (from the solidus to $F_{\max }$ ) within the various melting regimes shown in Figure 50. The dashed line shows the mean melts produced by a range in mantle temperature. The other lines trace the $\bar{P}$ and $\bar{F}$ characteristics of extractable melts within a single melting regime beneath the ridge. For example, incomplete focusing causes the low F ligh $\mathrm{P}$ melts in the melting regime not to be extracted, so all extracted melts lie along the lower portion of the standard model line. The thick line labeled "cold mantle" shows an equilibrium melting model for a colder mantle temperature.

from the top of each melting column. If extracted from the top of the melting column, then there is no variability in the endmember case, since all mantle rises to and equilibrates at the base of the crust (Figure $50 e$ ). In this case, the melt produced by active upwelling will occupy a single point along the standard model melt trajectory shown in Figure 52. In contrast, if melts are sampled from throughout the melting column, then melting must approach fractional melting, and the instantaneous melts will lie along the trajectory for fractional melting, shown in Figure 52.

\subsubsection{Implications}

The utility of the above exercise is to illustrate how simple predictions of the mean properties of the ocean crust and the P$F$ characteristics of melts within the melting regime can be calculated and inferred given a melting model and RMC. Each of the above models makes specific predictions of relationships among $\overline{\mathrm{F}}, \overline{\mathrm{P}}$ and crustal thickness for the global variability, and between $P$ and $F$ for local variability. We can estimate crustal thickness from seismic measurements, $\bar{F}$ from the concentration of moderately incompatible elements (like $\mathrm{Na}$ ), and $\overline{\mathrm{P}}$ from the concentration of elements with pressuredependent partition coefficients (like Fe). The new chemistry function can be used to calculate $\mathrm{Fe}$ and $\mathrm{Na}$ contents for the diversity of melting regimes. Therefore, by combining the physical models described above with the new chemistry 
function, we can directly compare the models to the data. Note that it is the combination of inferences concerning extent of melting, pressure of melting and crustal thickness that provides the most useful constraints. For example, active upwelling can produce thicker crust and greater mean extents of melting (lower $\mathrm{Na}$ ) relative to the standard model, but the mean pressures are lower than those that would be generated by increasing mantle temperature. Thus the use of all three parameters permits distinctions among models that could not be made on the basis of any two alone.

Several important results are clear at the outset. First, mantle temperature is the ONLY parameter investigated that produces positive relationships between $\overline{\mathrm{F}}, \overline{\mathrm{P}}$ and crustal thickness for mean crust, and therefore a predicted inverse relationship between $\mathrm{Na}_{8.0}-\mathrm{Fe}_{8.0}$ and between $\mathrm{Na}_{8.0}$ and crustal thickness. These are the principal characteristics of the global vector for MORB (Figures 15 and 18). Thus the qualitative conclusions of $K L 87$ and $M B 88$ still seem reasonable, and more complex melting regimes do not provide alternative explanations for the observed data. Furthermore, complex differentiation processes do not produce the requisite relationships among $\mathrm{Na}, \mathrm{Fe}$ and crustai thickness either (Appendix C).

Second, it is a natural consequence of pressure release melting that melts generated from within the melting regime have the opposite relationship between $F$ and $P$ compared to mean melts pooled from throughout the melting regime, as noted by $K L 89$. As a result, a variety of melting regimes should produce diverse magmas that range from low degree melts formed at high pressures to high degree melts formed at low pressures. Qualitatively, therefore, the cross-cutting "local trends" that occur on slow-spreading ridges appear to be consistent with a range of melts produced within a single melting regime. That said, it is also clear that the various types of melting regimes can differ substantially in the detailed character of these melts. An exact fit to the data holds the potential for better constraining the nature of the melting regime and the melting process. Precise values for local variations in crustal thickness, which are currently lacking, would be an invaluable aid in constraining the possibilities.

It is also likely that real melting regimes may embody combinations of the various isolated parameters discussed above. Hot mantle may have a larger melting regime and hence less efficient focusing. Fractional melting may promote passive upwelling, while equilibrium melting may promote active upwelling because the presence of melt will lower the viscosity and enhance the buoyancy of the upwelling mantle. These possibilities will need to be considered as we attempt to quantitatively reproduce the chemical characteristics of MORB.

Finally, this entire discussion has tacitly assumed a twodimensional ridge, and we will need to consider the relationship of the melting regime to ridge segmentation. Large transform offsets may have a significant thermal effect on the melting regime and the ascending mantle. Active upwelling could occur at segment centers, with a thick thermal lithosphere at segment edges. Thus difierent processes could affect different portions of a ridge segment and the chemical expression of local variability.

\subsection{The origin of the global vector}

Although mantle temperature qualitatively accounts for the global vector, the quantitative efforts by $K L 87$ and $M B 88 \mathrm{did}$ not reproduce the absolute values of MORB $\mathrm{Na}$ and $\mathrm{Fe}$ (see Figure 43). In addition, mantle heterogeneity has not been quantitatively evaluated as a possible explanation for the global vector. Albarede [1992] and Natland [1989] have suggested that much of the major element signal is associated with ridges affected by hot spots. Since hot spots are derived from an isotopically heterogeneous mantle, perhaps major element heterogeneity of the mantle is a possible cause of the global vector. The new melting model can be used to determine what combination of conditions can produce the global vector of composition and to test whether mantle heterogeneity might account for the global vector.

The calculated mantle melts all have significantly more than $8 \mathrm{wt} \% \mathrm{MgO}$, and the compositions formed near the solidus at the higher pressures have as much as $18 \mathrm{wt} \% \mathrm{MgO}$. Thus the calculated mantle melt compositions must be fractionated to $8 \% \mathrm{MgO}$ in order to compare them to the MORB data. This is problematic, for our calculations do not provide $\mathrm{CaO}$ or $\mathrm{Al}_{2} \mathrm{O}_{3}$ concentrations. $\mathrm{CaO}$ and $\mathrm{Al}_{2} \mathrm{O}_{3}$, however, can be reasonably estimated from the existing experimental data and, moreover, the effects of different amounts of $\mathrm{CaO}$ and $\mathrm{Al}_{2} \mathrm{O}_{3}$ within the permissible range do not seriously affect the crystallization results. The potential diversity of fractionation processes is a more serious problem. The crystallization pressure may vary with both crustal thickness and spreading rate, and the crystallization process may vary between an equilibrium, fractional, or in situ process [Langmuir, 1989]. Our evaluation of crystallization, therefore, must take into account some of the diversity of mechanisms by which crystallization may take place.

\subsubsection{Evaluation of Fractional and Equilibrium Melting Models}

The results of the new melting model for equilibrium melting were described above (section 5) and shown in Figure 47. Figure 53 compares the MORB array for normal ridges to calculated $\mathrm{Na}_{8.0}$ and $\mathrm{Fe}_{8.0}$ variations for mean melts from melting regimes that result from different mantle temperatures (different $P_{o}$ ). The figure shows both the calculated melt compositions, and these compositions adjusted to $8 \mathrm{wt} \% \mathrm{MgO}$ by fractional crystallization at 1 atm and at $4 \mathrm{kbar}$. As explored at length in Appendix C, for the same parental magma composition, high pressure crystallization leads to higher $\mathrm{Fe}_{8.0}$ than low pressure crystallization, but coes not cause a significant change in the $\mathrm{Na}_{8.0}$. The calculated melt compositions produced by equilibrium melting have $\mathrm{Na}_{2} \mathrm{O}$ and $\mathrm{FeO}$ contents that are too low to account for the global array (Figure 53a). After crystallization at $1 \mathrm{~atm}$, the melt compositions still do not have sufficient range in $\mathrm{FeO}$, and crystallization at $4 \mathrm{kbar}$ leads to too flat a slope. If the mantle $\mathrm{Na}_{2} \mathrm{O}$ abundance were increased by $30 \%$ (to $0.40 \mathrm{wt} \%$ ), then the melt array would intersect the MORB field, but the range in $\mathrm{FeO}$ would still be insufficient. Figure 54 compares the calculated $\mathrm{Na}_{8.0}$ and crustal thickness values to the MORB data. The $\mathrm{Na}_{8.0}$ 

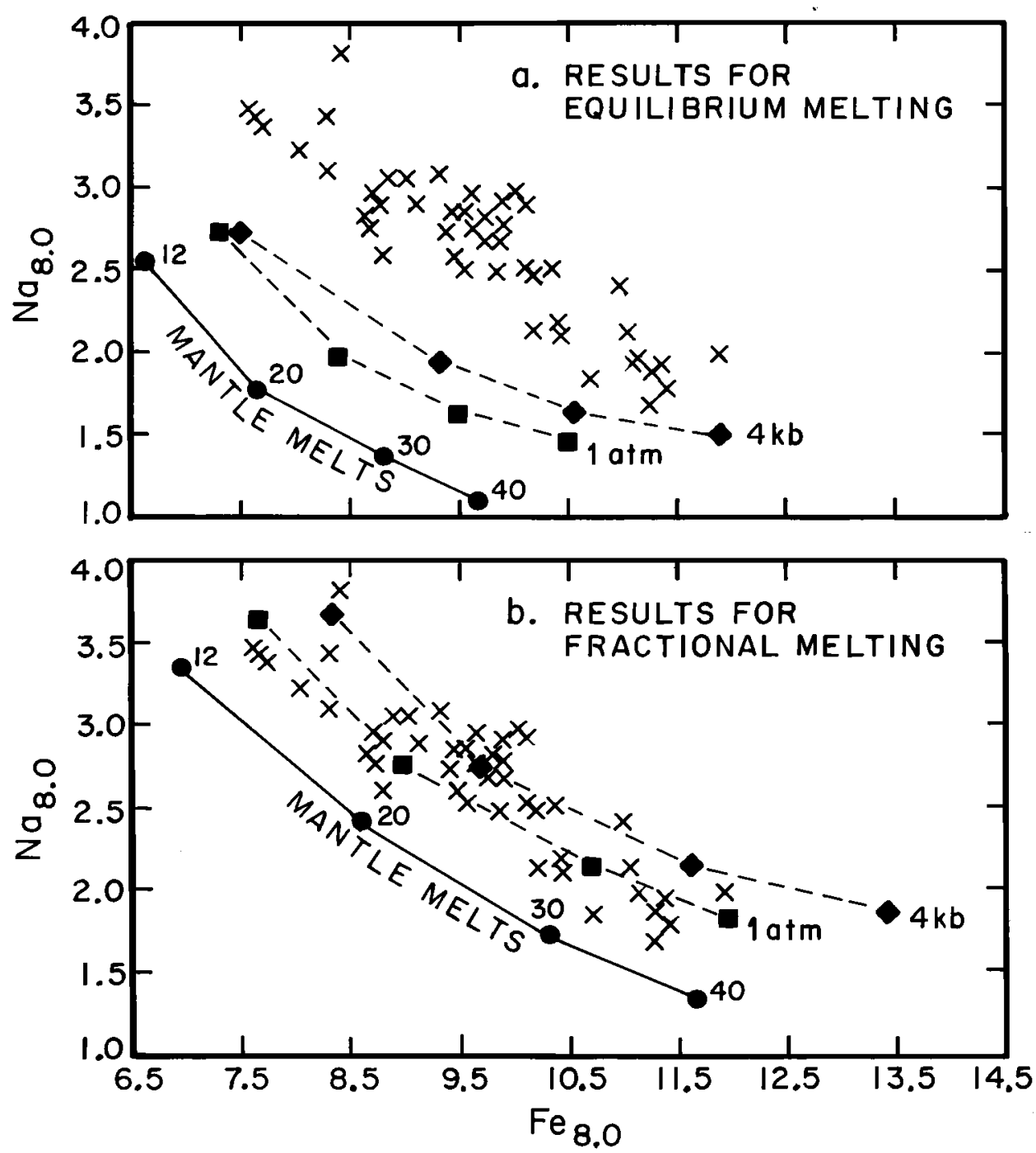

Fig. 53. $\mathrm{Na}_{8.0}$ vs. $\mathrm{Fe}_{8.0}$ for MORB, for calculated mantle melts and for the same melts fractionated at $8 \% \mathrm{MgO}$ at pressures of $1 \mathrm{~atm}$ and $4 \mathrm{kbar}$. The X's are normal MORB (from Figure 15). The top Panel (a) shows the results for equilibrium melting and the bottom Panel (b) for fractional melting for a range in $P_{0}$ from $12-40 \mathrm{kbar}$. The mantle melt curves are the same as those shown in Figures $47 \mathrm{~b}$ and $48 \mathrm{~b}$. Each labeled dot along the mantle melt curve is the mean melt for a given melting regime. Note that equilibrium melting gives neither high enough $\mathrm{Na}_{8.0}$ nor the right $\mathrm{Na8}-\mathrm{Fe} 8$ slope to account for the date.

values for pooled equilibrium melts are too low for the observed range in crustal thickness, but again, an increase in the $\mathrm{Na}_{2} \mathrm{O}$ concentration of the mantle source to $0.40 \mathrm{wt} \%$ would allow the calculations to overlap the data. Since this source abundance for $\mathrm{Na}_{2} \mathrm{O}$ is unreasonably high for the depleted or primitive mantle (see discussion in section 5.3 ), we conclude that equilibrium melting does not adequately reproduce the global MORB array. The more rigorous and completely independent quantitative modeling reported here, therefore, results in the same discrepancies for equilibrium melting that were apparent in our evaluation of the $K L 87$ and $M B 88$ mudels.

As discussed above (section 5.3), accunulated fractional melting leads to higher mean pressures of melting, and a greater range in mean pressure for a range in $P_{0}$. The compositional consequences of fractional melting are higher $\mathrm{Fe}_{8.0}$ contents for the same $\mathrm{Na}_{8.0}$ contents and a greater range in $\mathrm{Fe}_{8.0}$ for the same range of $\mathrm{Na}_{8.0}$ (Figures 47 and 48). After fractionation correction to $8 \mathrm{wt} \% \mathrm{MgO}$, the observed pooled fractional melts calculated from the new melting model are coincident with the MORB array (Figure $53 b$ ). The results for fractional melting also straddle the observations for $\mathrm{Na}_{8,0}$ and crustal thickness data (Figure 54). The detailed relationship between these calculated results and the observations depends in part on the parameters used for the calculation. Changing the mantle source concentration from $0.30 \mathrm{wt} \%$ to $0.26 \mathrm{wt} \%$ $\mathrm{Na}_{2} \mathrm{O}$ results in a curve that skirts the low side of the global array. Reducing the $1 \mathrm{~atm}$ intercept of solidus could also lower the $\mathrm{Fe}_{8.0}$ values by about $1 \%$ (see -igure 49). Certainly, 


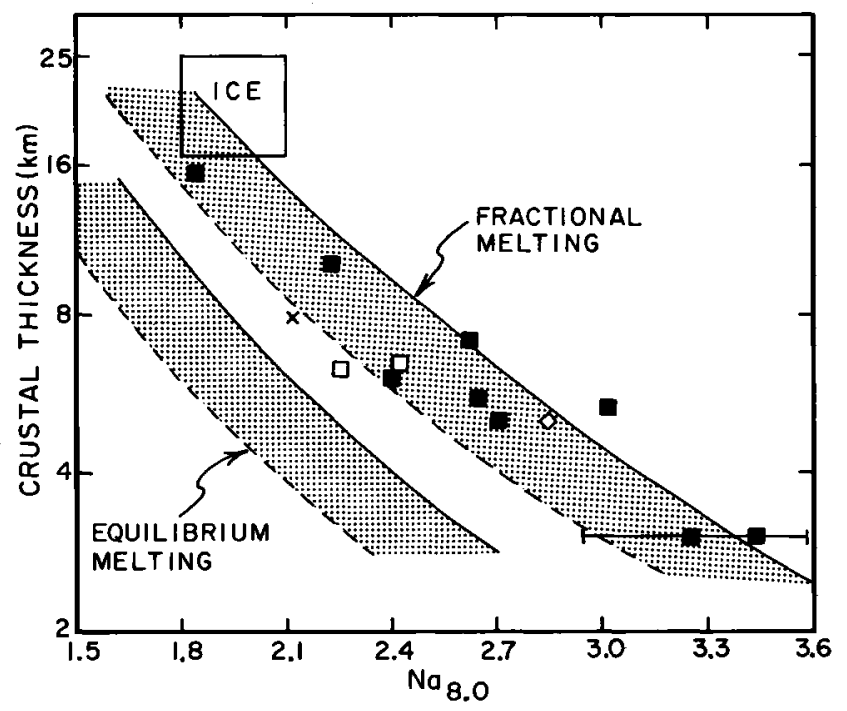

Fig. 54. $\mathrm{Na}_{8.0}$ and crustal thickness for observed ocean crust (from Figure 18) and calculated mean melts (from Figure 53). Dashed lines are for $\mathrm{C}_{0}$ of $0.26 \mathrm{wt} \% \mathrm{Na}_{2} \mathrm{O}$, and solid lines are for $\mathrm{C}_{0}$ of $0.30 \mathrm{wt} \%$ $\mathrm{Na}_{2} \mathrm{O}$. The equilibrium melting curves do not intersect the data field.

within the uncertainties of the parameters, the fractional melting calculations appear to reproduce the field of the observations more closely than the efuilibrium melting calculations.

Another test of the consistency of these melting models is whether they reproduce the $\mathrm{Ti}_{8.0}-\mathrm{Na}_{8.0}$ MORB data. Figure 55 compares MORB data with a curve calculated for fractional melting. Although $\mathrm{TiO}_{2}$ varied regionally (Figure 17), the calculated curve closely fits the data from normal North Atlantic ridges.

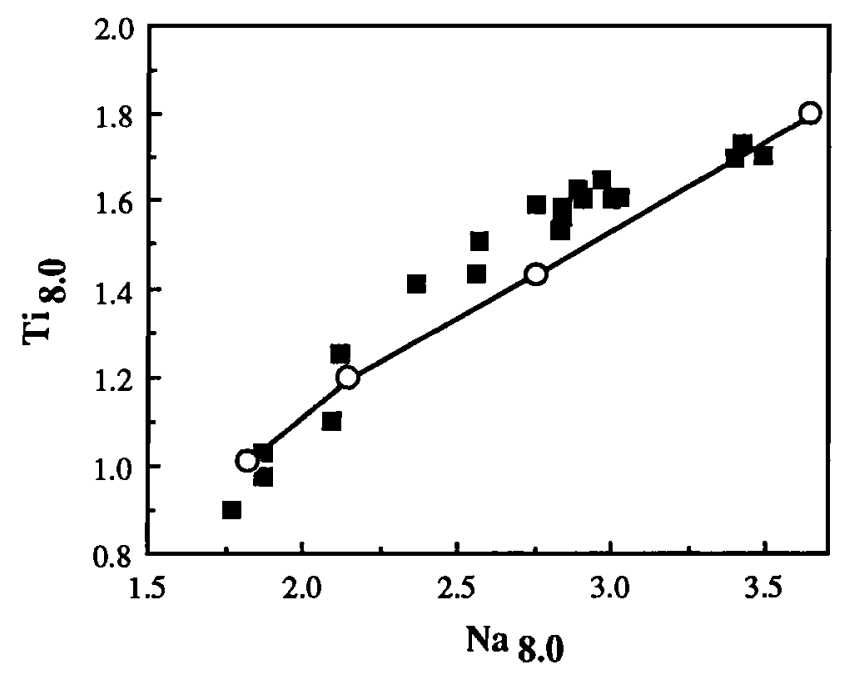

Fig. 55. Data from N. Atlantic ridges (from Figure 17b) are compared to calculated results for $\mathrm{Ti}_{8.0}$ and $\mathrm{Na}_{8.0}$. The mudel curve is for mean melts derived by fractional melting, with a cource concentration for $\mathrm{TiO}_{2}$ of $0.15 \mathrm{wt} \%$. Melts have been normalized to $8 \mathrm{wt} \% \mathrm{MgO}$ by fractional crystallization at 1 atm.
Fractional melting may also help to explain the discrepancy between the MORB data and the silica concentrations calculated by the $K L 87$ and $M B 88$ equilibrium models. Although the new quantitative model currently does not calculate $\mathrm{SiO}_{2}$, the higher mean pressures for fractional melting should lead to lower silica concentrations, in better accord with the MORB data (see Figure 43). The lower $\mathrm{SiO}_{2}$ contents obtained by fractional melting may also help to explain the lack of MORB compositions that are saturated with orthopyroxene under mantle conditions. Since the $\mathrm{SiO}_{2}$ is lower than predicted from equilibrium melting, orthopyroxene, which is preferentially stabilized by higher $\mathrm{SiO}_{2}$, may not appear as readily on the liquidus of accumulated melts produced by fractional melting.

In summary, these calculated results indicate that fractional melting models reproduce the global correlations very closely, while equilibrium melting models do not. These results are in remarkable agreement with arguments based on abyssal peridotites that have been made recently by Johnson et al. [1990]. A critical question, however, concerns the residual porosity that could be present during melting. Perfect fractional melting leads to $0 \%$ porosity, while equilibrium melting produces a porosity that increases with melt fraction during ascent of the mantle beneath the ridge, ultimately rising to values of $20-30 \%$. Langmuir et al. [1977] argued for a few percent residual porosity based on trace element modeling, while Johnson et al. [1990] have argued for a residual porosity of less than $1 \%$. The melting results presented here are not of sufficiently high resolution to constrain this problem well. Although equilibrium melting is ruled out for reasonable mantle source abundances of $\mathrm{Na}_{2} \mathrm{O}$, a continuous melting model with several percent residual porosity is permitted by the data. However, the perfect fractional melting model also is adequate.

\subsubsection{Evaluation of Mantle Heterogeneity as an Explanation for the Global Compositional Variations}

There are two aspects that need to be sonsidered in order to evaluate the effects of heterogeneity in the mantle source. First, we need to consider reasonable ways in which source compositions might vary on a regional basis, for if all elements are allowed to vary randomly, the problem is intractable. Second, the effects of source variations on the melting and chemistry functions need to be quantified.

Constraints on the source variations come from direct observation of mantle materials. Data from mantle nodules and peridotite massifs tend to exhibit linear trends on $\mathrm{MgO}$ variation diagrams [e.g., Frey et al., 1985; Maaloe and Aoki, 1977], an example of which is shown in Figure 56 for samples from the Ronda peridotite. Such trends are observed for all of the major elements that can be accurately determined, with the high $\mathrm{Na}_{2} \mathrm{O}$, low $\mathrm{MgO}$ samples showing high $\mathrm{SiO}_{2}, \mathrm{TiO}_{2}, \mathrm{CaO}$ and $\mathrm{Al}_{2} \mathrm{O}_{3}$ and slightly high $\mathrm{FeO}$. These observations place constraints on how probable sources for MORB can vary, and on the $\mathrm{Na}_{2} \mathrm{O}$ and $\mathrm{FeO}$ contents of undepleted mantle.

A curious feature of linear trends for $\mathrm{MgO}-\mathrm{Na}_{2} \mathrm{O}$ is that they are not consistent with an origin by different extents of partial melting. Partial melting leads to rapid depletion of $\mathrm{Na}_{2} \mathrm{O}$, 


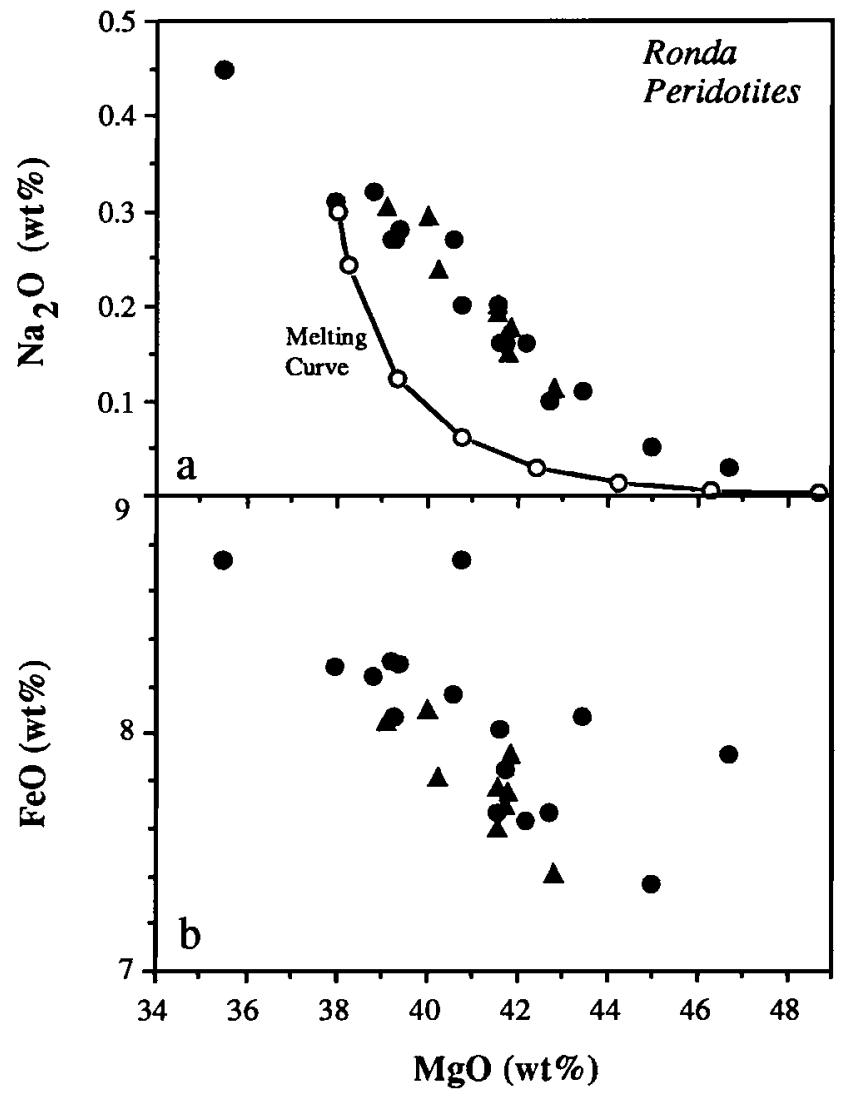

Fig. 56. $\mathrm{MgO}$ vs. $\mathrm{Na}_{2} \mathrm{O}$ and $\mathrm{FeO}$ for Ronda peridosites. Closed circles are data from Frey et al. [1985]. Closed triangles are data from Reisberg [1988] determined at Lamont by DCP on Ronda spinel peridotites. Also shown for $\mathrm{Na}_{2} \mathrm{O}$ is a calculated curve for changes in residual mantle composition as a function of increasing extents of melting. Note the peridotites are not explained as simple residues of equilibrium or fractional melting.

whether the melting process is equilibrium or fractional, and produces a concave upward curve on Figure 56a. Linear trends on variation diagrams are produced generally by mixing or unmixing. Therefore a simple interpretation of the observed trends is that they may result from either addition of basaltic material to a highly depleted mantle, perhaps in the form of subducted ocean crust, or by the extraction of a uniform melt composition to varying extents, as was suggested by Frey et al. [1985]. Despite these interesting complexities in the details of the depletion and enrichment process in the upper mantle, it is clear that the dominant control on major element variability is the relative amount of basaltic components that are in the mantle. Of course, another important aspect of mantle heterogeneity is the presence of ubiquitous veins in mantle peridotite. Such veins lead to a heterogeneous mantle on a small scale, and will be considered further in section 6.3.

The predominantly linear variations of the ultramafic data provide a range of compositions with which to evaluate major element heterogeneity. We use three compositions along the Ronda array as sources, one more depleted in basaltic constituents than the average mantle ( $41 \mathrm{wt} \% \mathrm{MgO}$ ), another more enriched (35.4 wt\% $\mathrm{MgO}$ ), and one of average mantle composition ( $38 \mathrm{wt} \% \mathrm{MgO}$ ). Values for all other elements can simply be taken from the linear data arrays.

Variations in mantle composition also produce a change in the temperature of the solidus: more fertile mantle has a lower solidus temperature at a given pressure. In order to take this effect into account, we have used a value of $20^{\circ} \mathrm{C}$ change in the solidus temperature per one percent change in the $\mathrm{Mg} \#$ of the mantle. This value was estimated from existing experimental data, although it is not well constrained.

A shift in the solidus due to a shift in $\mathrm{Mg \#}$ leads, further, to a change in the pressure of intersection of the solidus for an upwelling parcel of mantle (Figure 57). For example, a $1 \%$ decrease in the $\mathrm{Mg \#}$ causes the solidus to decrease by $20^{\circ} \mathrm{C}$, which leads to a $1.5 \mathrm{kbar}$ increase in $\mathrm{P}_{\mathrm{o}}$ (for a solidus slope of $13^{\circ} \mathrm{C} / \mathrm{kbar}$ ). These changes lead to variations in the extents and pressures of melting, which may counteract or accentuate the effects of varying source composition alone. For example, a lower $\mathrm{Mg \#}$ leads to higher $\mathrm{FeO}$ contents for a given pressure (simply from the $K_{D}$ relationship), and the lower solidus temperature leads to a greater $\mathrm{P}_{\mathrm{o}}$, which also contributes to higher $\mathrm{FeO}$ contents. Therefore the two effects enhance one another, and the $\mathrm{FeO}$ contents of melts from more fertile mantle will be higher. For $\mathrm{Na}_{2} \mathrm{O}$, however, the effects of source composition and change in extent of melting counterbalance. The higher $\mathrm{Na}_{2} \mathrm{O}$ in the source enriches the melts, but this is partially offset by the increased amount of melting due to the greater $P_{0}$. For a $1 \%$ change in $\mathrm{Mg}$, the source changes substantially in $\mathrm{Na}_{2} \mathrm{O}$, yet the solidus intersection differs by only $1.5 \mathrm{kbar}$, which causes only a small change in the extent of melting. Thus the change in the source concentration for $\mathrm{Na}_{2} \mathrm{O}$ dominates the dilution caused by greater melting.

These qualitative considerations show that source heterogeneity can be expected to lead to a positive correlation between $\mathrm{FeO}$ and $\mathrm{Na}_{2} \mathrm{O}$ contents. This conclusion is rather insensitive to the uncertainties in the temperature-composition relations of the solidus. For example, if there were no change in solidus temperature with composition, $\mathrm{FeO}$ and $\mathrm{Na}_{2} \mathrm{O}$ contents would still both increase, because the source concentrations increase. Therefore the slope of $\mathrm{Na}-\mathrm{Fe}$ variations caused by mantle heterogeneity is well constrained in sign if not magnitude.

Quantitative Modeling. The new calculations permit a more rigorous evaluation of mantle heterogeneity, given appropriate input parameters. The calculations considered above showed the range of compositions produced by a range of mantle temperatures acting on a single source composition. Now we consider the consequences of a single mantle temperature acting upon a range of source compositions. Figure 58 shows the calculated variations in melt compositions produced by melting of the three different mantle compositions for a mantle temperature that would intersect a normal solidus at $20 \mathrm{kbar}$. In agreement with the qualitative discussion above, the more enriched mantle (lower $\mathrm{Mg} \#$ ) produces a pooled melt with higher $\mathrm{Na}_{2} \mathrm{O}$ and somewhat higher $\mathrm{FeO}$ contents. The enrichment in $\mathrm{FeO}$ is limited, because the increase in alkalis 


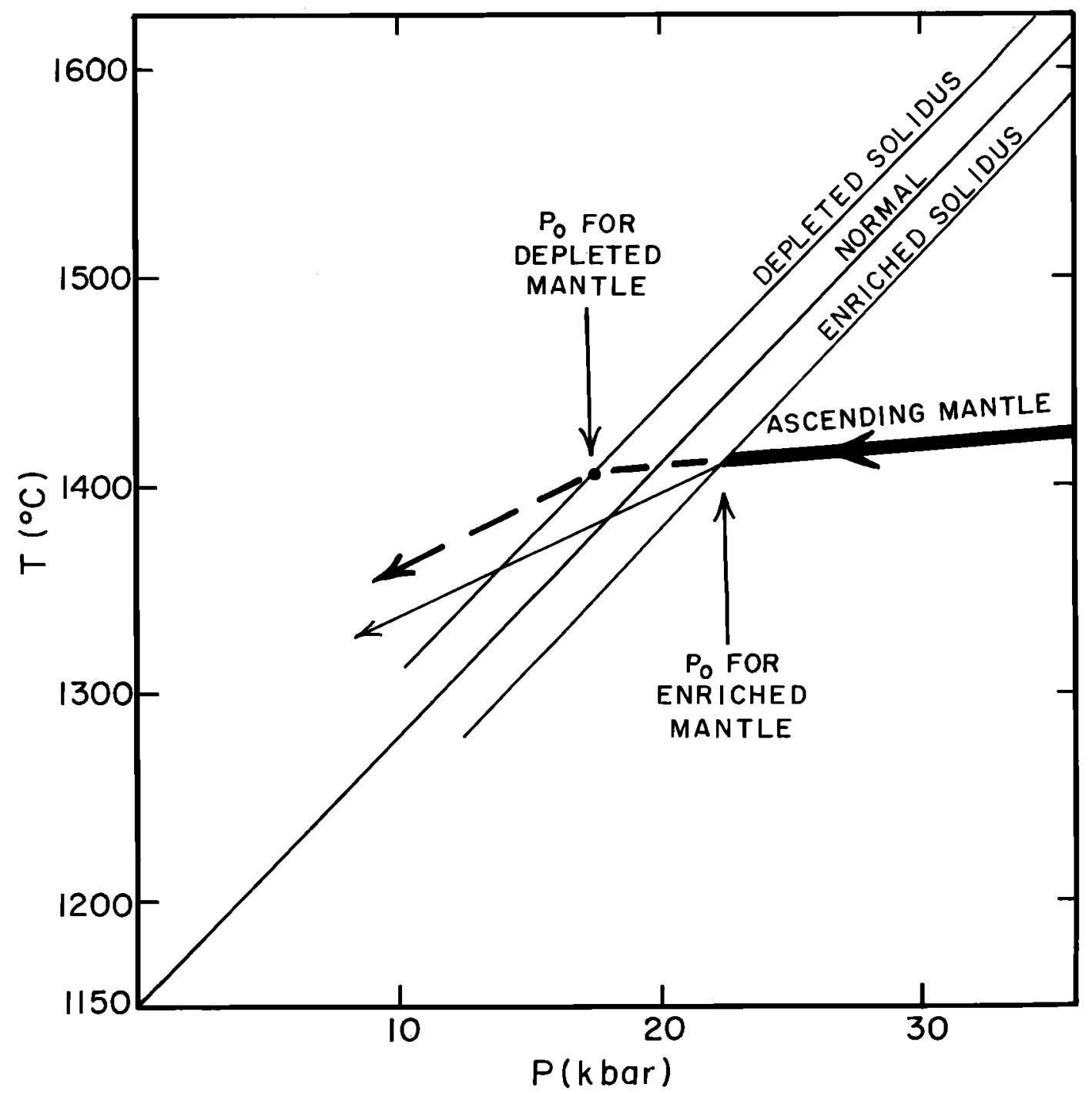

Fig. 57. P-T diagram illustrating schematically the consequences of changing mantle composition on melting, for a mantle temperature that would intersect the solidus at a pressure of $20 \mathrm{kbar}$ for nomal mantle. Enriched mantle has a lower solidus temperature which causes the pressure of intersection of the solidus $\left(P_{0}\right)$ to be greater. Since melting starts deeper, more pressure release is possible, and the overall extent of melting is slightly higher. The combination of more fertile mantle and deeper melting leads to higher overall FeO contents. But the enriched mantle has substantially more $\mathrm{Na}_{2} \mathrm{O}$, which is not completely compensated for by the slightly greater melting extents. Therefore, for a given mantle temperature, melts of enriched mantle have higher $\mathrm{FeO}$ and higher $\mathrm{Na}_{2} \mathrm{O}$ than melts of depleted mantle.

increases the $\mathrm{FeO} \mathrm{K}_{\mathrm{d}}$ 's for olivine. Because the enriched mantle starts melting deeper and melts more, it also produces a greater quantity of melt, and hence a thicker section of oceanic crust (Figure 59). These results, although illustrated for a single mantle $T$, apply to the whole range of mantle temperatures. For example, temperature variations acting on relatively enriched mantle would lead to curves offset to higher $\mathrm{Na}_{2} \mathrm{O}$ and $\mathrm{FeO}$ relative to normal mantle. These calculations demonstrate that mantle heterogeneity in the absence of mantle temperature variations leads to slopes that are opposite those of the global MORB data. Thus mantle heterogencity, as observed in ultramafic nodules and peridotite massifs, cannot be the primary cause of the global correlations.
Heterogeneity could contribute to the observations, however. The width of the global correlations could result in part from mantle heterogeneity. This may be particularly important for some hot spots. For example, Iceland is slightly offset to the high $\mathrm{Na}_{8.0}-\mathrm{Fe}_{8.0}$ side of the global correlations, while the Azores and Jan Mayen are offset substantially to the low side (Figure 15). Iceland also has relatively thicker crust, while the Azores region has relatively thinner crust, in comparison to the melting calculations for normal mantle presented in Figure 54. Thus Iceland could be derived from a mantle that is slightly more fertile than normal mantle, leading to higher $\mathrm{Na}_{8.0}$ and $\mathrm{Fe}_{8.0}$ and thicker crust. The Azores region, on the other hand, could result from melting of depleted mantle. 


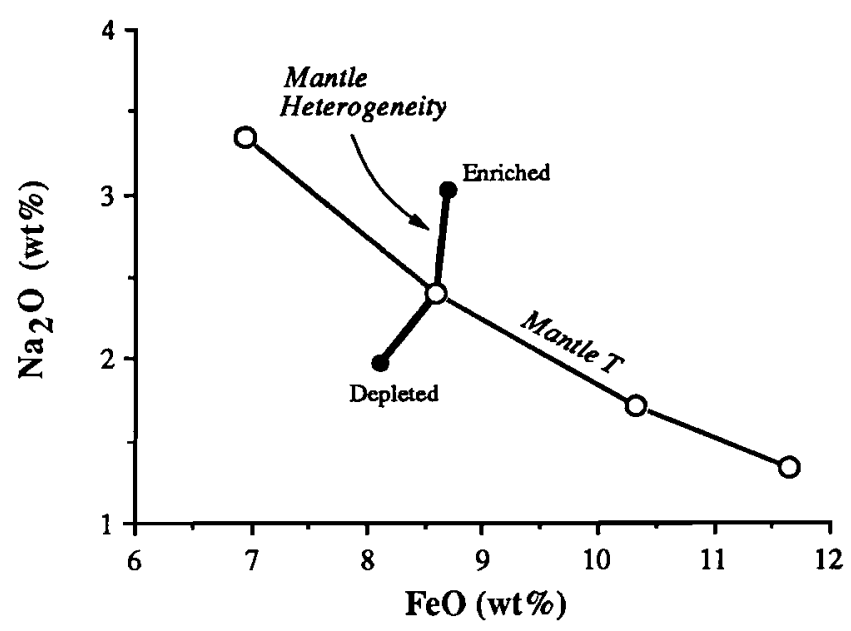

Fig. 58. Comparison of quantitative results for mantle temperature variations acting on a homogeneous mantle, with constant mantle temperature acting on a heterogeneous mantle. The mantle temperature curve is reproduced from Figure 53. The mantle heterogeneity curve is produced at the temperature that intersects the solidus of nomal mantle at $\mathrm{P}_{\mathrm{o}}$ of $20 \mathrm{kbar}$. Enriched, normal and depleted mantle have, respectively: 36,38 , and $41 \mathrm{wt} \% \mathrm{MgO} ; 8.6,8.4$, and $7.9 \mathrm{wt} \% \mathrm{FeO}$; $0.4,0.3$, and 0.22 wt $\mathrm{Na}_{2} \mathrm{O}$; and $0.02,0.012$ and $0.006 \mathrm{wt} \% \mathrm{~K}_{2} \mathrm{O}$. Note that constant temperature acting on a heterogeneous mantle produces arrays of melts that are roughly perpendicular to the global correlations observed in MORB.

Of course, the most incompatible elements are highly enriched in the Azores region. Thus, these calculations suggest trace element enrichment by a volatile(?) phase of a source that was previously depleted in its major element components. This explanation for the Azores might also account for the anomalous relationship between depth, $\mathrm{Na}_{8.0}$ and $\mathrm{Fe}_{8.0}$ that is observed approaching some hot spots (e.g. Figure 19). The lower $\mathrm{Fe}_{8.0}$ would be the result of the depleted major element matrix of the mantle, and possibly the higher volatile contents during melting, while the higher $\mathrm{Na}_{8.0}$ contents would result from the more recent enrichment event. This contrast between the Azores and Iceland is consistent with previous discussions of distinct origins for the sources of these two hot spots [Langmuir and Hanson, 1980; Schilling et al., 1983].

\subsubsection{Conclusions with Respect to the Global Variability of MORB Compositions}

Based on the modeling above, we can conclude that:

1. The global correlations for normal ridges continue to be well explained by variations in mantle temperature acting on a relatively homogeneous mantle.

2. The melting process does not appear to be equilibrium melting. Pure fractional melting (0\% residual porosity during melting) is consistent with the data, but there are significant uncertainties that would permit a melting model intermediate between fractional and equilibrium melting. For example, if the mantle is slightly more enriched than our normal mantle composition, the data would fall between the results for fractional and equilibrium melting. These ur.certainties make it difficult to quantify the percent of residual porosity during melting from the basalt data alone.

3. Mantle heterogeneity, such as is observed in ultramafic nodules and peridotite massifs, cannot reproduce the global correlations.

4. The offset of some hot spots from the global correlations may result from major element heterogeneity in the mantle.

5. Back-arc basins show similar global systematics to other ocean ridges, but are systematically offset to lower $\mathrm{Fe}_{8.0}$ and $\mathrm{Ti}_{8.0}$. These offsets cannot currently be modeled quantitatively due to the lack of knowledge of the effects of small amounts of water on mantle and basalt phase equilibria. Suppression of plagioclase during crystallization could lead to lower calculated values of $\mathrm{Fe}_{8.0}$ and $\mathrm{Ti}_{8.0}$ [see Fryer et al.., 1990], but the effects of water on $\mathrm{FeO}$ during melting are not yet known.

\subsection{The Origins of Local Major Element Variability}

We tum next to explore the origin of the local vectors of chemical variability. This is a difficult undertaking due to the large number of factors that could play a ro! $\epsilon$ in the diversity of ridge environments. However, the regularity of the major element trends on slow- and fast-spreading ridges is striking, and should have a straightforward and consistent explanation. A key issue is whether the local variability (corrected for low pressure crystallization) results from melting processes, crystallization processes, or small scale mantle heterogeneity. In the following, we discuss how the data and quantitative modeling shed light on the problem.

$K L 89$ focussed primarily on the origin of the Atlantic-type local chemical variations, and proposed that they were controlled by different extents of melting as individual or

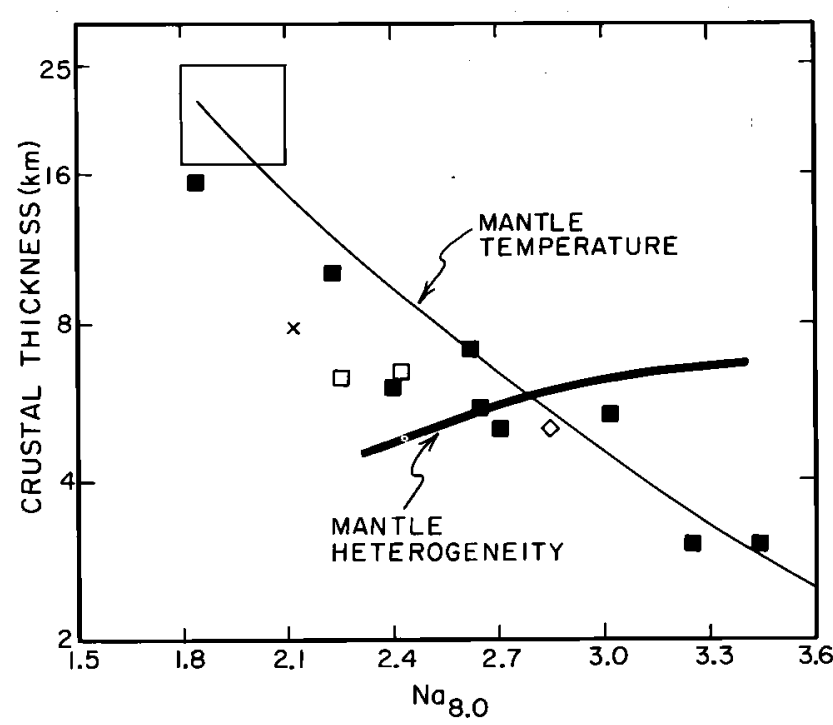

Fig. 59. Crustal thickness results for the same conditions described in Figure 58. Enriched mantle is more fertile and melts more, leading to both thicker crust and higher $\mathrm{Na}_{8.0}$. As in Figure 58 , the heterogeneity model produces results that are essentially perpendicular to the observations. Crustal thickness data from Figure 18. 
partially pooled melts were sampled from the melting regime. Melts produced by smaller extents of melting would be sampled from just above the solidus and would show the greatest pressure of equilibration (hence, higher $\mathrm{Na}_{8.0}$ and $\mathrm{Fe}_{8.0}$ and lower $\mathrm{Si}_{8.0}$ ). $K L 89$, however, did not quantitatively explore the origin of the local vector, but rather showed a qualitative comparison between the Atlantic-type trends and the general trends for polybaric equilibrium melting inferred from the experimental data. They also noted that they could not rule out an origin by high-pressure fractionation, but this issue was not dealt with in depth. At the short course, Kinzler and Grove used data from new experiments and calculations to point out the potential importance of high-pressure fractionation in controlling the slow-spreading local vector [Kinzler and Grove, $1992 a$ and $b$; Grove et al., this volume]. Yet a third explanation for local variability comes from studies of trace elements and isotopes, and from the recognition of ubiquitous small-scale heterogeneities in mantle peridotites. Melting of a "veined mantle" as called upon for the trace element and isotope models [e.g., Hanson, 1977; Wood, 1979; Zindler et al., 1984; Prinzhofer et al., 1989] could lead to substantial local major element variability as well.

\subsubsection{Quantitative Evaluation of Melting Models}

In section 5 , the new quantitative melting function was used to calculate the $\mathrm{FeO}$ and $\mathrm{Na}_{2} \mathrm{O}$ contents of melts produced by adiabatic equilibrium and fractional melting within a given melting regime (Figures 47 and 48 ). We also showed above (section 6.2) that the global MORB array could be explained as an array of mean melt compositions produced by pooling fractional melts from throughout melting regimes of different solidus pressures (different mantle temperatures). Thus the success of the quantitative modeling in reproducing the global vector depends on the pooling of instantaneous melts. This, in turn, leads to predictions for the chemical characteristics of local variations, if those variations are caused by the enuption of melts from different parts of a single melting regime.

In Figure 60, the curved trajectories show the compositions of instantaneous melts produced within the melting regime for different pressures of intersection of the solidus, for both fractional and equilibrium melting corrected to $8 \% \mathrm{MgO}$. The paths of the individual melts cut across the global vector of mean melts at a high angle, as suggestec by $K L 89$. These calculated melting curves are compared to the local trends for slow-spreading ridge segments. It is clear that the calculated paths do not correspond with the shallow slopes of the MORB data. The fit is particularly poor for the fractional melting model, where the individual melts from the melting regime have substantial variation in $\mathrm{Na}_{2} \mathrm{O}$ but very little variation in $\mathrm{FeO}$. Thus while the global vector appears to be quantitatively consistent with the pooling of melts from different melting regimes, the slow spreading local vector is not well modeled by melts from within a melting regime. Of course, melting column effects are a completely inadequate explanation for the Pacific-type local vector, where $\mathrm{Fe}_{8.0}$ and $\mathrm{Na}_{8.0}$ correlate inversely (Figure 24), because calculated melts that occur within a melting regime form steep positive trends (Figure 60).
Modification of the mixing function to account for different upwelling mechanisms and patterns does not appear to aid in explaining the slow-spreading local trend. This conclusion results because different mixing functions control primarily how much of each melt along a given melting path, of given $P_{o}$, contributes to the final mean melt. For example, active upwelling causes all the melts to be made tip of the high $F$ (low $\mathrm{Na}_{2} \mathrm{O}$ ) portions of the path, while inefíicient melt focusing causes melts from the low $\mathrm{F}$ (high $\mathrm{Na}_{2} \mathrm{O}$ ) part of the path to remain unsampled (Figure 52). Since all the melting paths in Figure 60 have little variation in $\mathrm{FeO}$ and much in $\mathrm{Na}_{2} \mathrm{O}$, no combination of melts along them can produce the shallow slope of the slow-spreading local trend. In the case of a thick thermal boundary layer, both the mixing and melting functions are affected. The highest $F$ melts along the melting path are not produced, and the cooling effects of the boundary layer can cause less melting over a given pressure interval, leading to greater pressure changes for a given melting increment (Figure 52). This can produce fractional melting paths with positive slopes on a $\mathrm{Na}_{2} \mathrm{O}-\mathrm{FeO}$ diagram, but the slopes are still too steep to account for the slow-spreading trends. Thus, for a given $P_{o}$ none of the melting models we have investigated reproduces the observed $\mathrm{Na}-\mathrm{Fe}$ slope of the local trends. Alternative explanations for the local vectors must be sought.

\subsubsection{Complex Fractionation Models}

One alternate explanation for the origin of the Atlantic-type local vector is that it results from either high-pressure crystallization [KL89; Kinzler and Grove, 1992a and b; Grove et al., this volume], or some complex low-pressure crystallization process. These ideas have intuitive appeal, because crystallization processes should be strongly affected by spreading rate. For example, at slower spreading rates, cooling is more efficient, and crystallization might take place at deeper levels. Furthermore, the balances among frequency of injection, eruption and cooling in periodically replenished magma chambers (PRMC) could logically be expected to vary with spreading rate. Fortunately, these processes can be modeled quite well for basaltic systems, so we can evaluate their efficacy in creating the local vectors. The modeling methods and effects of high-pressure crystallization and complex low-pressure crystallization are ciscussed in detail in Appendix C. Here we present the more important results that forward the general line of discussion.

To evaluate the various models, it is useful to recognize that the local trends, originally defined by the correlations among $\mathrm{Na}_{8.0}, \mathrm{Fe}_{8.0}$ and $\mathrm{Si}_{8.0}$, are also clearly observed in the $\mathrm{Na}_{2} \mathrm{O}, \mathrm{FeO}$ and $\mathrm{SiO}_{2}$ abundances for the many samples from the FAMOUS area with approximately $8 \mathrm{wt} \% \mathrm{MgO}$ (Figure 23), and that for these samples there are additional correlations among $\mathrm{Na}_{2} \mathrm{O}$ and $\mathrm{CaO}, \mathrm{CaO} / \mathrm{Al}_{2} \mathrm{O}_{3}, \mathrm{TiO}_{2}$ and $\mathrm{K}_{2} \mathrm{O}$. The existence of these systematics for many elements at a single value of $\mathrm{MgO}$ simplifies conceptually how we test the fractionation models: we seek processes that can produce the observed variability at 8 $\mathrm{wt} \% \mathrm{MgO}$ without worrying about subsequent low-pressure fractionation corrections. 


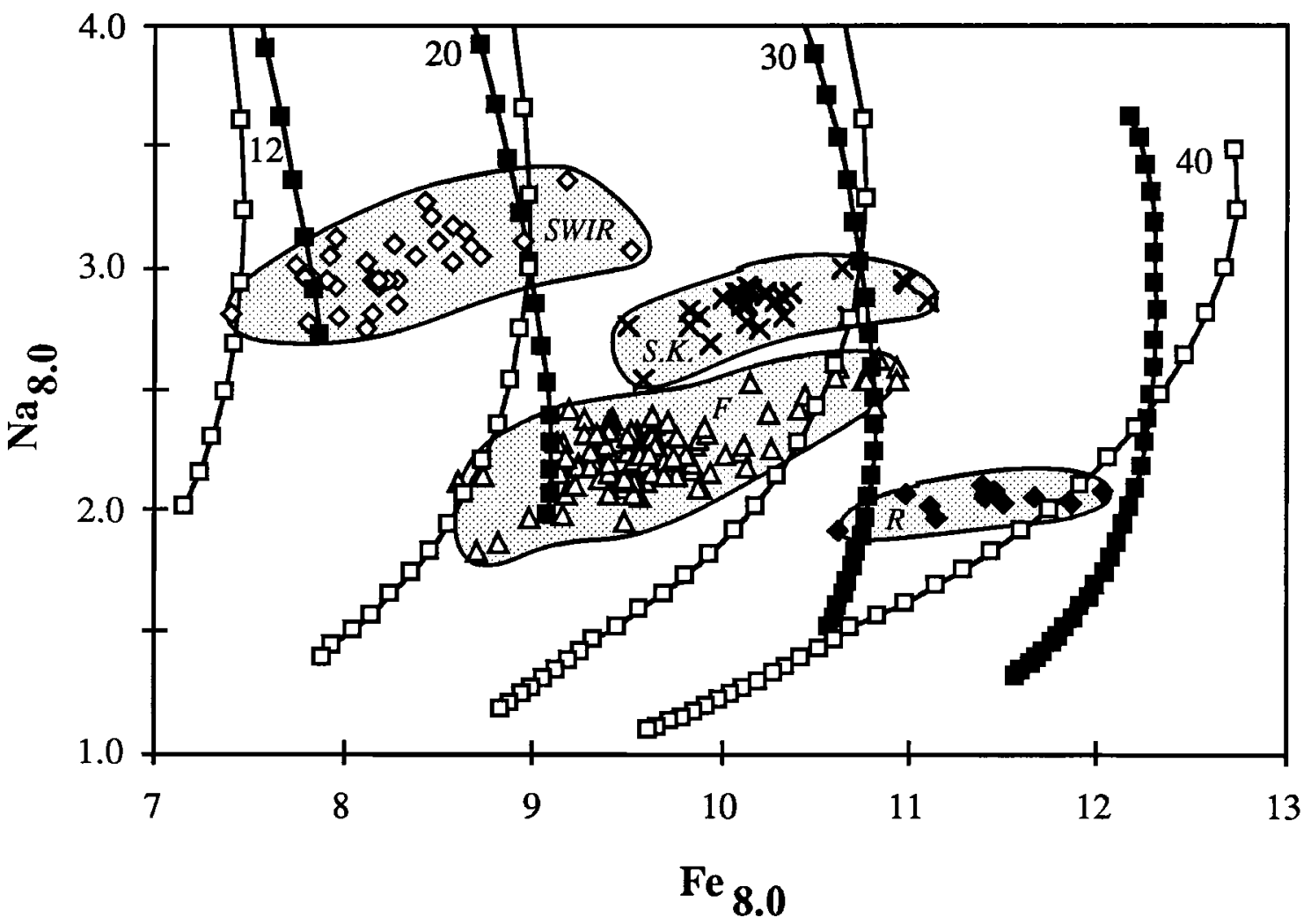

Fig. 60. Melting path curves for fractional (solid squares) and equilibrium (open squares) melting. Each path shows the liquid compositions in the melting regime, with the high $\mathrm{Na}_{2} \mathrm{O}$ liquids forming at depth near the solidus, and the low $\mathrm{Na}_{2} \mathrm{O}$ liquids forming near the base of the crust. Each point on the curves represents a pressure drop of 1 kbar. Curves are corrected for 1 atm fractionation based on the calculated correction for the mean melts (Figure 53). The fields on the diagram are for several regions which exhibit the known range in slow-spreading local vectors from Figure 21: $R$ (Reykjanes Peninsula), F (FAMOUS area), SK (S. Kane Fracture Zone), SWIR (AII Fracture Zone).

Low-pressure Fractionation. As discussed in detail in Appendix $C$, mixing of evolved and primitive magmas by either in situ fractionation or in periodically replenished magma chambers can, under certain circumstances, lead to associated enrichments of both $\mathrm{FeO}$ and $\mathrm{Na}_{2} \mathrm{O}$ at a given value of $\mathrm{MgO}$ (the two models differ substantially, however, in their effects on trace elements). Although these processes cannot produce the EPR local trend, they might account for the positive correlation between $\mathrm{Fe}_{8.0}$ and $\mathrm{Na}_{8.0}$ of the slowspreading local data.

In general, the steady-state melts produced by periodically replenished magma chambers lie between fractional and equilibrium crystallization trends (Figure $\mathrm{Cl}$ ) as discussed in Appendix $\mathrm{C}$, and thus do not produce large variations in either $\mathrm{FeO}$ or $\mathrm{Na}_{2} \mathrm{O}$ at $8 \mathrm{wt} \% \mathrm{MgO}$. The one exception is if very high $\mathrm{MgO}$ liquids ( $>12 \mathrm{wt} \% \mathrm{MgO}$ ) mix with very fractionated liquids, such that the mixture lies outside the closed system crystallization field, producing large co-variations in $\mathrm{FeO}$ and $\mathrm{Na}_{2} \mathrm{O}$ at $8 \mathrm{wt} \% \mathrm{MgO}$ (Figure $\mathrm{C} 2$ ). However, for the FAMOUS samples, this would also produce a positive correlation between $\mathrm{Na}_{2} \mathrm{O}$ and $\mathrm{SiO}_{2}$ at $8 \mathrm{wt} \% \mathrm{MgO}$, as well as an inverse correlation between $\mathrm{Na}_{2} \mathrm{O}$ and $\mathrm{Al}_{2} \mathrm{O}_{3}$. In contrast, one of the hallmarks of the MAR-type local vector is the inverse correlation between $\mathrm{Na}_{8.0}$ and $\mathrm{Si}_{8.0}$ (Figures 23 and 25). The FAMOUS data also show no correlation between $\mathrm{Na}_{2} \mathrm{O}$ and $\mathrm{Al}_{2} \mathrm{O}_{3}$ (Figure 23) in contrast to the expected effects of such mixing processes.

Furthermore, as discussed in Appendix C, such mixed magmas have olivine alone on the liquicus just after mixing and evolve rapidly back to the multi-phase liquid line of descent of the parental magma. In order to observe the strong $\mathrm{FeO}-\mathrm{Na}_{2} \mathrm{O}$ enrichment, these magmas musi be sampled just after mixing. Many of the FAMOUS samples, however, are saturated with both olivine and plagioclase at $8 \mathrm{wt} \% \mathrm{MgO}$, not olivine alone [e.g., Bender et al., 1978]. This co-saturation is reflected by the consistent, positive slope that samples follow on a plot of $\mathrm{MgO}$ vs. $\mathrm{Al}_{2} \mathrm{O}_{3}$.

The difficulties in reproducing the slow-spreading local vector by periodically replenished magma chamber processes also apply to in situ differentiation. Crystallization of plagioclase and pyroxene, which produces an increase in $\mathrm{FeO}$ and $\mathrm{Na}_{2} \mathrm{O}$ contents, is also expected to lead to an increase in $\mathrm{SiO}_{2}$ and a decrease in $\mathrm{Al}_{2} \mathrm{O}_{3}$. These explanations, therefore, would produce systematics that are not observed in the MAR- 
type local vector and require special conditions that are not consistent with the widespread occurrence of the slowspreading local trend. As discussed below, trace element systematics argue against an origin by low-pressure crystallization processes. This is not to say that PRMC or in situ crystallization processes do not occur, but rather that they do not appear to produce satisfactory explanations for the characteristics of either the MAR-type or EPR-type local vectors.

High Pressure Fractionation. At slower spreading centers such as the Mid-Atlantic Ridge, where the lithospheric cap may be thicker and where there is no evidence for crustal magma reservoirs, crystallization may occur at higher pressures. As discussed in Appendix C, crystallization at higher pressures leads to increased stability of clinopyroxene such that it may precede the arrival of plagioclase on the liquidus [e.g., Bender et al., 1978]. Enhanced clinopyroxene crystallization leads to an inverse correlation between $\mathrm{Na}_{2} \mathrm{O}$ and $\mathrm{CaO} / \mathrm{Al}_{2} \mathrm{O}_{3}$, and early crystallization of the three-phase assemblage olivineplagioclase-clinopyroxene leads to early enrichments in $\mathrm{FeO}$, $\mathrm{Na}_{2} \mathrm{O}$ and $\mathrm{TiO}_{2}$. Thus, at least qualitatively, the observed chemical co-variations that characterize the MAR-type local vector are consistent with high-pressure fractionation. A potential difficulty, however, is that the expected enrichment in $\mathrm{Na}_{2} \mathrm{O}$ is moderated by the more albitic plagioclase that crystallizes at higher pressures [e.g., Bender et al, 1978; Fram and Longhi, 1991].
In order to quantitatively evaluate whether high-pressure fractionation can account for the variability seen in the local trends, we modified the $1 \mathrm{~atm}$ liquid line of descent program of Weaver and Langmuir [1990] to include pressure dependencies for the distribution coefficients, as described in Appendix C and shown in Table $\mathrm{Cl}$. The program was calibrated to the available experimental data on FAMOUS compositions. Using a primitive composition similar to that of the FAMOUS region, calculations were carried out at 0,4 and $8 \mathrm{kbar}$ in order to compare the compositions calculated at $8 \mathrm{wt} \% \mathrm{MgO}$ to the local trends seen in the FAMOUS/AMAR data (Figures 61, C5, and C6; see also results in Table C2.)

In qualitative terms, crystallization at 0,4 and $8 \mathrm{kbar}$ produces magma compositions at $8 \mathrm{wt} \% \mathrm{MgO}$ that are similar to the local trends observed among the FAMOUS/AMAR samples with $\sim 8 \mathrm{wt} \% \mathrm{MgO}$ (see Figure 61 and Appendix $\mathrm{C}$ ). In detail, however, there are a number of problems with the model. For example, the observed range in $\mathrm{Na}_{2} \mathrm{O}$ in the FAMOUS/AMAR data is almost a factor of three greater than that produced by the high pressure modeling (Figure 61). This is in part a result of the pressure effect on plagioclase composition, but even if the crystallizing plagioclase at 8 kbar were less albitic, fractionation at 8 kbar could only produce about $50 \%$ of the total observed range in $\mathrm{Na}_{2} \mathrm{O}$. Similarly, high-pressure fractionation only produces about half of the total range in $\mathrm{TiO}_{2}$, despite the fact that the total amount crystallized by $8 \mathrm{wt} \% \mathrm{MgO}$ at $8 \mathrm{kbar}$ is approximately $50 \%$.
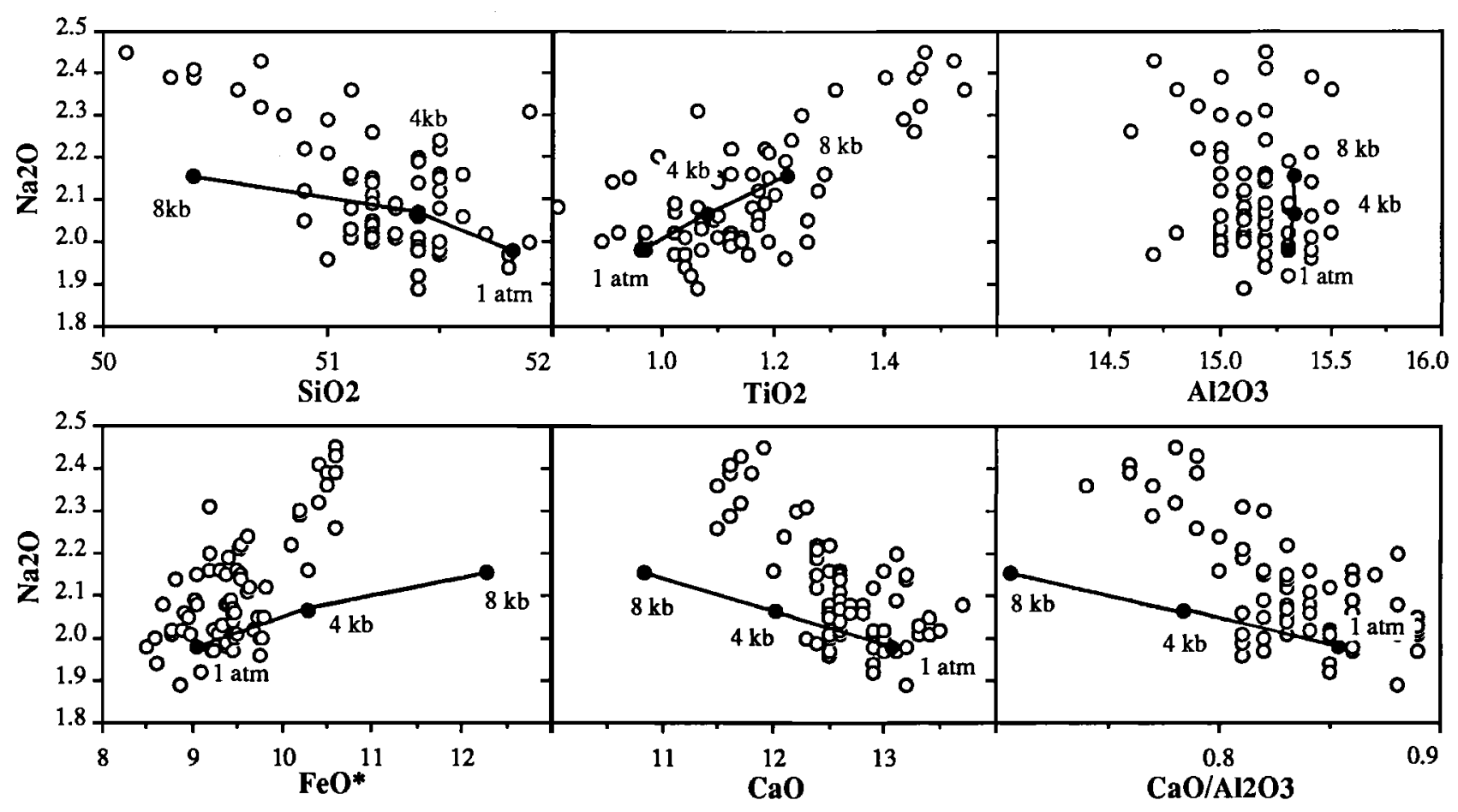

Fig. 61. Data from the FAMOUS/AMAR region with $8 \mathrm{wr} \% \mathrm{MgO}$ (open symbols, reproduced from Figure 23) compared to calculated liquid compositions at $8 \mathrm{wt} \% \mathrm{MgO}$ (solid symbols connected by lines) for crystallization at 1 atm, $4 \mathrm{kbar}$, and $8 \mathrm{kbar}$. These liquid compositions are calculated are from the LLDs shown in Figure C5 (see Appendix C for details). 
Other high-pressure fractionation models (such as Model 2 considered in Appendix C) encounter similar difficulties in reproducing the local trends observed in the FAMOUS data. Thus, although it is logical that high-pressure fractionation may occur at slow-spreading ridges, and may contribute to the variability observed, it does not provide a quantitative explanation for the slow-spreading local vector. In general, it should also be recognized that high-pressure fractionation, like melting, has physical constraints associated with it. Highpressure fractionation will occur only if there is a thick lithosphere beneath the ridge, or if melts pass through the lithosphere above the melting regime far from the ridge prior to being focussed towards the ridge. It also seems likely that the melt would react with the cooler mantle rather than crystallize liquidus minerals in isolation from the mantle [e.g., Keleman, 1990]. Models of high pressure fractionation also need to be consistent with the melting process. For example, if fractionation occurs at $8 \mathrm{kbar}$, the meltir.g column can extend only to a pressure of $8 \mathrm{kbar}$. High pressure fractionation, therefore, implies lower extents of melting, higher mean pressures of melting, and substantially thinner crust for a given mantle temperature.

\subsubsection{Mantle Heterogeneity and the Local Vector}

Ultramafic rocks show different types of heterogeneity on a variety of scales. One type of heterogeneity results from different amounts of removal or addition of mafic compositions, as modeled above for Ronda peridotite compositions (section 6.2.2) A second type of heterogeneity is veining, which is common in peridotites over distances of $\mathrm{cm}$ to tens of meters. These veins could represent simple modal variations of the same mineral composition as the host peridotite, or they could be of a different composition than the host peridotite. Indeed, both types of mantle heterogeneity, bulk compositional and veining, could be present over the hundred $\mathrm{km}$ distances in which we find t.e local vectors of variability, so we discuss each type of heterogeneity in turn.

Variations in bulk mantle composition were considered as an explanation for the global vector and il'ustrated in Figure 58. For a given mantle temperature, the variations in mantle composition considered produce a trend of mean melts that cross-cuts the global vector. The melting column paths for each of the mantle compositions would be a group of curves, each of which would have large changes in $\mathrm{Na}_{2} \mathrm{O}$ for little change in $\mathrm{FeO}$. Melts mixed from these various melting paths could produce a diversity of compositions, but again $\mathrm{Na}_{2} \mathrm{O}$ varies too much and $\mathrm{FeO}$ varies not enough to explain the slowspreading type local variations. If a greater change in $\mathrm{Mg \#}$ for a given $\mathrm{Na}_{2} \mathrm{O}$ content is assumed, it would be possible to produce slopes that correspond better to the slow-spreading local vector, but there is no independent evidence for this type of heterogeneity.

Melting of a veined mantle is more difficult to treat quantitatively, because the heterogeneities may occur on a small scale, there is a wide range of possible compositions, and there is little experimental data on non-standard mantle compositions. The two types of veins (mineralogical concentrations versus distinctly enriched material) are also likely to have very different effects.

Veins of pyroxene with the same composition as the host peridotite will serve as foci of melting. The solidus temperature will be the same as the surrounding peridotite, but substantially more melting will take place in the vicinity of the veins over a given temperature interval, because clinopyroxene is the dominant constituent of the melt. This principal is clear from consideration of any phase diagram: the closer the melt composition and the solid composition, the more melting that occurs for a given temperature change simply because of the lever rule. Now consider elements such as $\mathrm{Na}$ and $\mathrm{Ti}$, which in the mantle are contained almost exclusively in pyroxene. At low extents of melting, the bulk partition coefficient is the same in the veins as elsewhere, because the liquid composition is simply $C_{d} / D$. Both $C_{o}$ and $D$ are proportional to the amount of pyroxene present, so the ratio is independent of the amount of $\mathrm{p}_{j}$ roxene. Thus the initial liquid composition is essentially independent of modal variations. As the extent of melting increases, however, there are large differences, and the abundances of $\mathrm{Na}$ and other elements contained primarily in clinopyroxene will be much greater in the liquid for a given extent of melting (Figure 62). Thus, in the melting of mantle veins there are two counterbalancing effects: higher $\mathrm{Na}_{2} \mathrm{O}$ for a given extent of melting, balanced by greater overall extents of melting. In terms of $\mathrm{FeO}$ variations, melting of clinopyroxene-rich veins can be expected to lead to lower concentrations of iron in the melt due to the lower concentrations of $\mathrm{Fe}$ and $\mathrm{Mg}$ in clinopyroxene relative to olivine. The higher $\mathrm{Na}_{2} \mathrm{O}$ in the melt also tends to decrease the $\mathrm{FeO}$ contents of the melt low because of the $\mathrm{K}_{\mathrm{d}}$ effect. If we choose arbitrary melting functions, we can arrive at liquid compositions that vary by about $0.5-1 \%$ in $\mathrm{FeO}$ with small changes in $\mathrm{Na}_{2} \mathrm{O}$, but the exact slope of the trends is not well constrained. Therefore reasonable evaluation of this model is still premature.

Enriched Veins: A Cause of the EPR Local Trends? A second type of vein is one that may have quite ciifferent mineralogy and chemical composition from the surrounaing mantle. Such veins have often been called upon as an explanation for variable degrees of trace element and isotope enrichment in local provinces [e.g. Prinzhofer et al., 1989; Karsten, 1990; Michael et al., 1989]. Quantitative modeling of such material is not yet possible, but there are some indications that these veins may be important in controlling ce.tain aspects of local variability. Current evidence suggests to us that such veins may cause the EPR local systematics identified in section 3.5 .2 , but probably are not important factors in the origin of the slow-spreading local trends.

One important characteristic of the EPR local trend is the association between major element composition and enrichment in incompatible trace elements (e.g., K/Ti). The variations in incompatible trace elements are far greater than can be explained by melting alone. In addition, Klein et al. [1986] found correlations between $\mathrm{Na}_{8.0}$ and $\mathrm{Fe}_{8.0}$ and the ${ }^{87} \mathrm{Sr} /{ }^{86} \mathrm{Sr}$ isotopic composition of basalts from some segments 


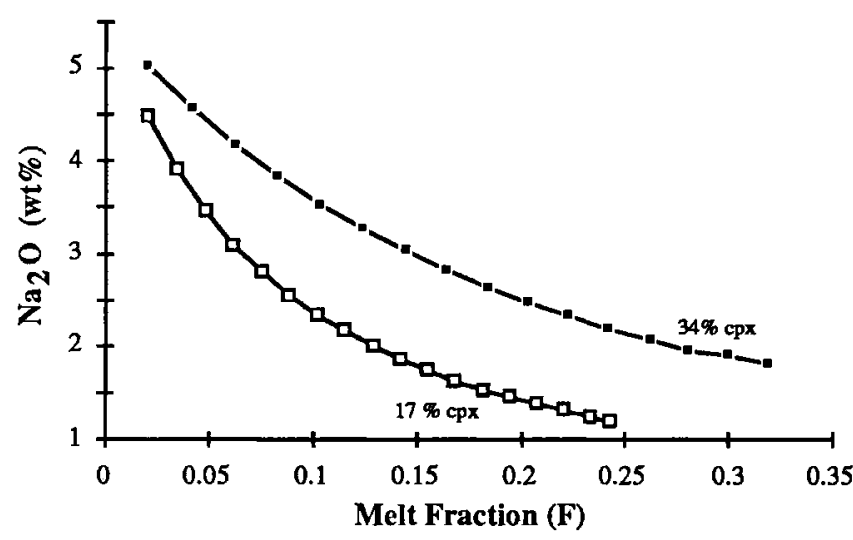

Fig. 62. Melting paths illustrating a possible effect of mineralogical heterogeneity during melting. The solid squares are for mantle with twice the amount of clinopyroxene (and twice the source concentration of $\mathrm{Na}_{2} \mathrm{O}$ ). The two curves are similar at low $\mathrm{F}$ because the ratio of $\mathrm{C} d \mathrm{D}$ is similar. At higher $F$, the mantle with twice the amount of clinopyroxene produces melts richer in $\mathrm{Na}_{2} \mathrm{O}$.

segments of the northern EPR (Figure 63). Thus, we have evidence for heterogeneity in trace elements and isotopes, and clear correlations between indices of this heterogeneity and the major element signal. Furthermore, the presence of diverse lava types over very short distances and times [e.g., Thompson et al., 1985; Langmuir et al., 1986; Sinton et al., 1990; Hekinian et al, 1989; Reynolds et al., 1992] suggests that a range of mantle source compositions is present at any one time beneath the EPR axis. This observation seems most consistent with intermixed sources of material, such as a network of enriched veins, and suggests that the EPR local trend could result from such small-scale mantle heterogeneity. This would be consistent with arguments made previously on the basis of trace elements and isotopes [e.g. Prinzhofer et al, 1989].

If so, the correlation of trace element or isotopic enrichment with major element variations leads to interesting but perplexing implications regarding the melting behavior of these veins. The major element characteristics of these enriched melts are the association of lower $\mathrm{Fe}_{8.0}$, lower $\mathrm{Si}_{8.0}$, with higher $\mathrm{Na}_{8.0}$ and $\mathrm{Al}_{2} \mathrm{O}_{3}$. These characteristics are not easily explained by the existing experimental data. It is important to recognize, however, that the enriched melts also tend to have higher volatile contents, particularly higher $\mathrm{H}_{2} \mathrm{O}$ contents [Michael, 1988]. Thus, the major element characteristics of this enriched end-member may result from melting under conditions of high volatile abundances. Quantitative testing of the various aspects of this hypothesis for the EPR local trend must await further experimental data on the appropriate bulk compositions in the presence of water.

While the link between trace elements, isotopes and major elements suggests that the EPR local trend results from source heterogeneity, the lack of such a link for the local trends in the Atlantic suggests that the slow-spreading local trend does NOT arise from enriched veins. In part, the contrast between the EPR and slow-spreading local trends alone indicates that a similar explanation will not account for both of them.

Shirey et al. [1987] analyzed an extensive suite of samples from just north of the Oceanographer Fracture Zone. These samples were recovered from a small area, and hence reflect local variability. The data exhibit large variations in isotopic composition and incompatible element abundances, so there is source heterogeneity. If source heterogeneity were controlling the local major element systematics, there should be a correlation between trace element enrichment and major element variations.

Major element data for these samples (Figure 21) have clear local vectors of the slow-spreading type. The local vectors exist for the samples that have similar $\mathrm{Ce} / \mathrm{Yb}$ ratios, that is, for subsets of the data that could be produced by different extents of melting. There is no correlation between trace element enrichment and position along a local vector for the data as a whole, as one might expect if the major element vector were controlled by small-scale heterogeneity. Therefore there is not the relationship between major and trace elements that is apparent on the EPR, and mantle heterogeneity does not appear to produce the local trend. The local trend can be present for subsets of the data where no heterogenetiy is required. Therefore mantle heterogeneity is an important aspect of this data set, but it is not the control on the local major element variability.

\subsubsection{The Slow-Spreading Local Vector Problem}

We have a reasonable hypothesis for the origin of the EPR local trend, although no quantitative model to account for its

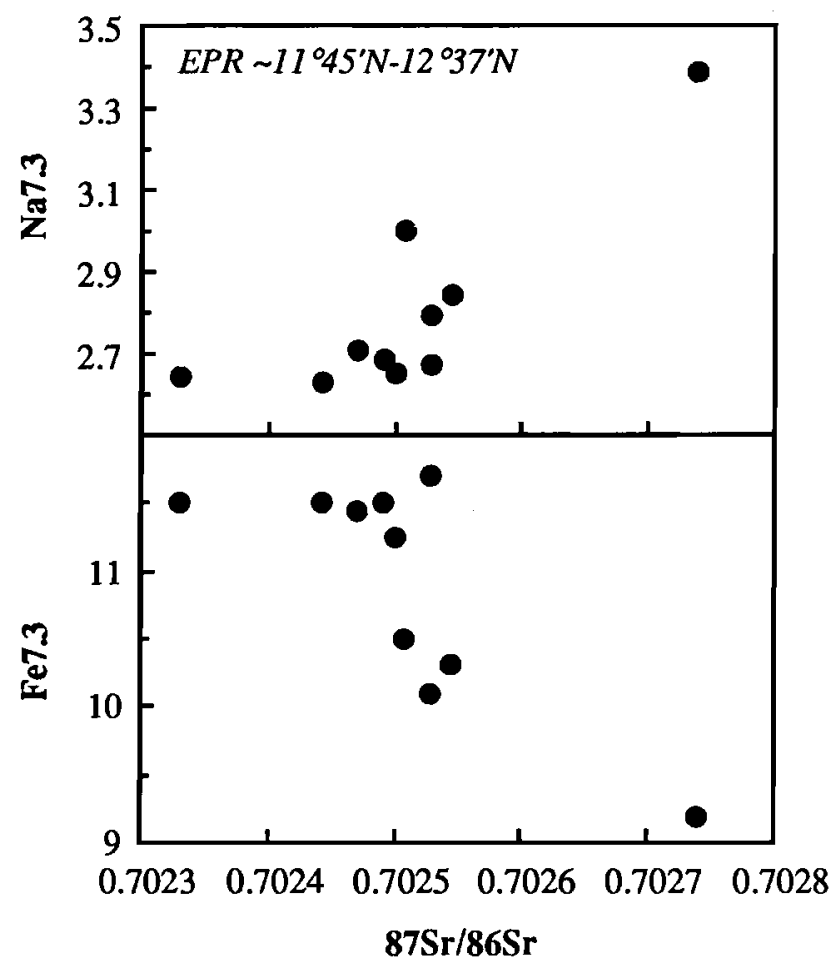

Fig. $63 .{ }^{87} \mathrm{Sr} /{ }^{86} \mathrm{Sr}$ vs. $\mathrm{Na}_{7.3}$ and $\mathrm{Fe}_{7.3}$ for EPR b.asalts from $\sim 11^{\circ} 45 \mathrm{~N}$ $12^{\circ} 37^{\prime} \mathrm{N}$. These correlations suggest that the high $\mathrm{Na}_{7.3}$, low $\mathrm{Fe}_{7.3}$ basalts are associated with a more enriched source. These high $\mathrm{Na}_{7.3}$. low $\mathrm{Fe}_{7.3}$ basalts are of the type that controls the EPR local vector. Data are from Langmuir et al. [1986, and unpublished], Klein et al., [1986], and Castillo et al. [1992, in preparation]. 
diverse aspects. We do not yet, however, have a satisfying explanation for the slow-spreading local vector. Although some melting and fractionation processes produce qualitatively similar results that may contribute to the chemical variations, none of them fully accounts for the observations. We are thus faced with a puzzle: the pooled melts of the melting regime account remarkably well for the global systematics of regional averages, yet the local variations that go into the regional average compositions do not have a quantitative model to account for them. Furthermore, these local systematics are a remarkably robust feature of the data, for they appear along ridge segments representing the total range of axial depth, in different ocean basins, and in the presence or absence of hot spot influences. Therefore, whatever their origin, it must be a robust aspect of the crust formation process.

Although we do not have a solution to the slow-spreading local vector, there are other aspects of the data that are relevant, and hint at the direction of a solution. In the following, we examine other lines of evidence that appear to shed light on the problem.

\subsubsection{Trace Element Evidence}

Fractionation up to pressures of about $10 \mathrm{kbar}$ involves the crystallization of plagioclase as well as pyroxenes and olivine. Plagioclase has a high partition coefficient for the element Sr. When plagioclase is a stable crystallizing phase, the bulk partition coefficient for $\mathrm{Sr}$ is about 1 , so $\mathrm{Sr}$ concentrations change very little as crystallization proceeds. However, during mantle melting $\mathrm{Sr}$ behaves as an incompatible element because plagioclase is not likely to be a stable mantle phase over most of the melting interval. Sr thus provides a key to evaluating whether the local vector is created by melting or crystallization processes. Sr is not the only important trace element in this evaluation, however. Melting in general is better able to produce large variations in incompatible elements. Therefore, trace elements as a group potentially can constrain the origin of the local vector.

Carrying out such tests requires abundant major and trace element data on samples, preferably glasses, from individual ridge segments where a local vector is well-developed. Unfortunately, there are few ridge segments for which we can explore this relationship. In the following, we examine data from three regions on the Mid-Atlantic Ridge to see if the local vectors observed in these regions are likely to be caused by melting or crystallization processes.

Langmuir et al. [1977] presented major and trace element data on hand-picked glasses from the FAMOUS region that spanned a substantial compositional range. Among them are two samples with $8 \mathrm{wt} \% \mathrm{MgO}$ that lie at the two ends of the local vector for the FAMOUS area discussed at length above. These two samples have substantially different abundances of all incompatible trace elements (varying by more than a factor of two), and Sr shows the same variability as other elements of similar incompatibility during mantle melting. This general behavior of $\mathrm{Sr}$ as an incompatible element is present for other FAMOUS data as well, as seen in Figure 64a. These large Sr
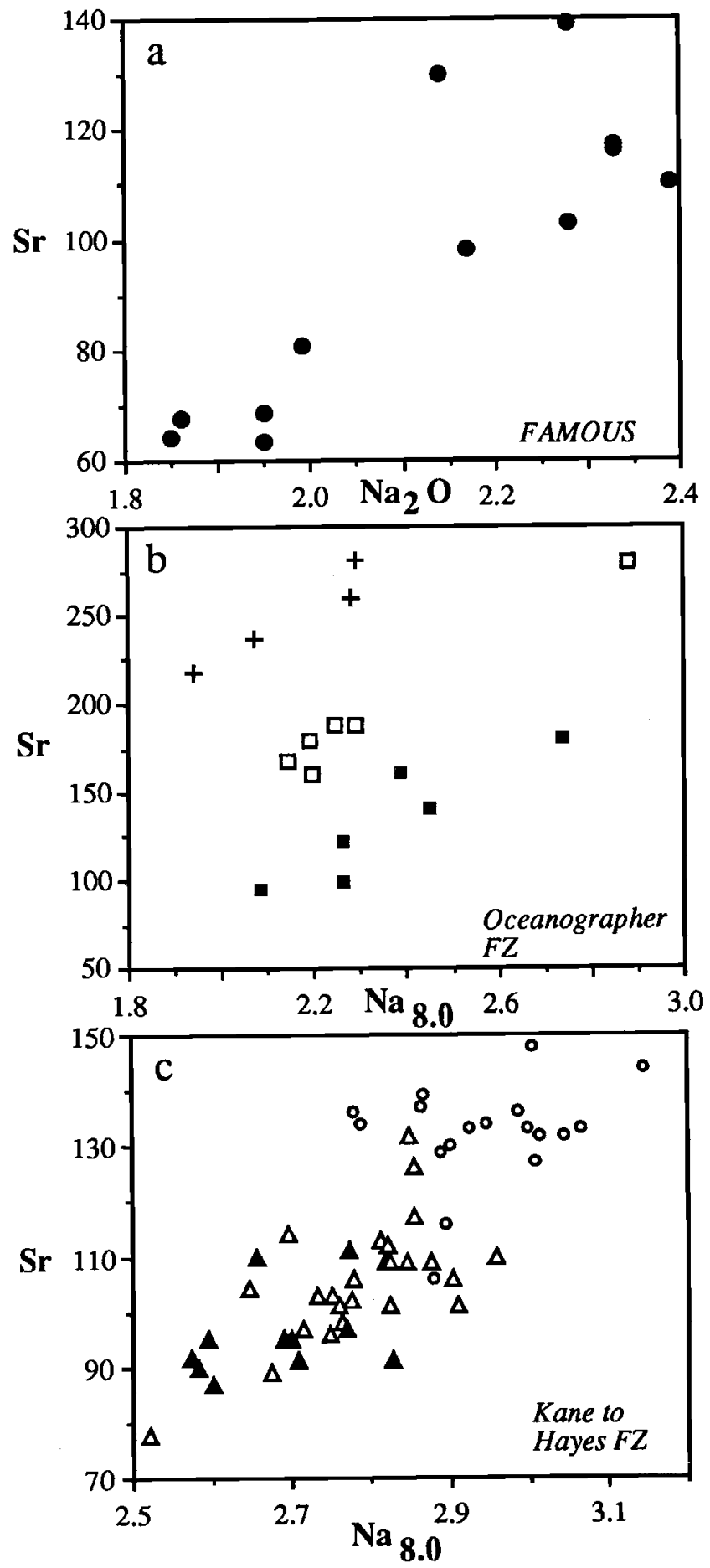

Fig. 64. $\mathrm{Na}_{80}$ vs. $\mathrm{Sr}$ for MORBs from various regions on the Mid Atlantic Ridge. a) High-MgO ( $8.5 \mathrm{wt} \%$ ) samples from the FAMOUS region, obtained by plasma emission specirometry [Langmuir, region, obtained
unpublished]. $b$ ) Samples from near the Oceanographer Fracture Zone.
Plusses for $\mathrm{Ce} / \mathrm{Sm}=9.7-10.7$; open squares for Ce/ $\mathrm{Sm}=7.4-9.1$; solid Plusses for $\mathrm{Ce} / \mathrm{Sm}=9.7-10.7$; open squares for $\mathrm{Ce} / \mathrm{Sm}=7.4-9.1$; solid
squares for $\mathrm{Ce} / \mathrm{Sm}=4.2-6.5$. Within each group, $\mathrm{Na}$ and $\mathrm{Sr}$ correlate positively. [Langmuir and Bender, unpublished data.] c) Samples collected between the Kane and Hayes FZs on the M,iid-Atlantic Ridge. Open circles $\left(23^{\circ} \mathrm{N}-24^{\circ} \mathrm{N}\right)$; open triangles $\left(27^{\circ} \mathrm{N}\right.$ to the Atlantis $\left.\mathrm{FZ}\right)$; filled triangles (Atlantis FZ to Hayes FZ). Analyses by plasma emission spectrometry [Langmuir, unpublished]. 
variations preclude a process in which plagioclase is a major fractionating phase, and suggest control by mantle melting.

A second slow-spreading local-trend data set comes from a region of normal ocean ridge basalts, where there is no hot spot trace element signature, between the Kane and Hayes Fracture Zones on the Mid-Atlantic Ridge. This region is of particular interest, because there is a substantial gradient in axial depth, from more than 4000 meters near the Kane Fracture Zone, to about 2500 meters south of the Hayes Fracture Zone. The data for $\mathrm{Na}_{8.0}-\mathrm{Fe}_{8.0}$ were shown in Figure 21a; Figure 64c shows the $\mathrm{Na}_{8.0}-\mathrm{Sr}$ relationships. The $\mathrm{Na}_{8.0}-\mathrm{Fe}_{8.0}$ diagram shows that the chemical variability is reflected in both global and Atlantic-type local vectors. The shallowest region has the highest $\mathrm{Fe}_{8.0}$ and lowest $\mathrm{Na}_{8.0}$, but the data for each local region exhibit a positive correlation between $\mathrm{Na}_{8.0}$ and $\mathrm{Fe}_{8.0}$.

$\mathrm{Na}_{8.0}$ and $\mathrm{Sr}$ correlate positively for this region (Figure $64 \mathrm{c}$ ). The correlation is obvious for the data as a whole, but this reflects in part the global component of variability. For each local region, there is still a positive correlation between the two parameters, but there is also significant variability in $\mathrm{Na}_{\mathbf{8 . 0}}$ for a given $\mathrm{Sr}$ content. This is particularly true for the southernmost region, near the Kane Fracture Zone. This data set as a whole may therefore require a two-fold explanation, one to account for the positive correlation between $\mathrm{Na}_{8.0}$ and $\mathrm{Sr}$, and another to explain the diversity of $\mathrm{Na}_{8.0}$ at a single small range of Sr contents.

The third region is near the Oceanographer Fracture Zone. Figure $64 b$ shows the $\mathrm{Na}_{8.0}$-Sr variations for this region, with the samples divided into three groups on the basis of their $\mathrm{Ce} / \mathrm{Yb}$ ratios. Within each group, there is the positive correlation that would be expected from melting processes. The component of heterogeneity appears to move sample groups to increasing $\mathrm{Sr}$ abundances for a given $\mathrm{Na}_{8.0}$. Thus, in this diagram there is clear evidence for a separation of the local trend from control by mantle heterogeneity, and also the positive correlation between $\mathrm{Na}_{8.0}$ and $\mathrm{Sr}$ that is characteristic of slow-spreading local trends as a whole.

The positive correlation between $\mathrm{Sr}$ and $\mathrm{Na}_{8.0}$ in each of these regions suggests that the slow-spreading local trend is produced by melting processes and not by crystallization processes. Neither low-pressure crystallization, no matter how complex, nor high-pressure crystallization (to about $10 \mathrm{kbar}$ ) can be the dominant control on the slow-spreading local vector of variability, based on this correlation. This conclusion is possible because of the ubiquitous occurrence of plagioclase as a crystallizing phase from MORB at low to moderate pressures. The data also suggest that the slow-spreading local trend is not produced by local mantle heterogeneity. Therefore this robust signature of slow-spreading ridges is teling us some important characteristics of the melting process, even though we do not yet have a quantitative model to account for it.

This is not to say that high-pressure fractionation plays no contributing role. For the region near the Zane Fracture Zone, Sr does stay approximately constant, which supports highpressure fractionation models that have buen suggested for this region [Tormey et al., 1987; Grove et al., 1990; Reynolds and
Langmuir, 1992 in press]. But high-pressure fractionation cannot be the root cause of the slow-spreading local vector.

\subsubsection{Evidence from Ocean Islands: Tou ard a Solution to the Local Vector Problem?}

One of the characteristics of the compusitional dependencies of the partition coefficients incorporated in the quantitative melting model is that high alkali contents lead to low FeO contents. Indeed, small-degree melts from deep in the melting column do have very low $\mathrm{FeO}$ contents in our calculated models. There is another rock series that has often been thought to result from low extents of meling at high pressures - the alkaline rocks found on ocean sslands and continents, regions where there is often a hot spoi an' thicker lithosphere that causes melting to take place at dceper levels. These rocks, some of which contain ultramafic nodules and must have ascended rapidly from the mantle, can have $7 \mathrm{wt} \%$ total alkalis at high $\mathrm{MgO}$ contents. Therefore, if the calculations are pertinent to the melting process, one would expect that these very alkaline rocks, particularly those with both high $\mathrm{K}_{2} \mathrm{O}$ and high $\mathrm{Na}_{2} \mathrm{O}$, would have relatively low $\mathrm{FeO}$ contents.

But in contrast to the calculations, these alkaline rocks are often exceptionally high rather than low in FeO. Not only are alkaline rocks generally high in $\mathrm{FeO}$, but the more alkaline the rock the higher its $\mathrm{FeO}$ contents. This is illustrated for rocks from the Hawaiian islands in Figure 65. In general, ocean island data for fresh volcanics exhibit a positive correlation between $\mathrm{FeO}$ and $\mathrm{Na}_{2} \mathrm{O}$, like the slow-spreading local vector, suggesting there is a "local vector" for ocean island volcanics as well as ocean ridge volcanics. For the ocean island rocks, the case for an origin by partial melting is also very strong, particularly since ultramafic xenoliths can occur in the alkaline rocks. The rocks also show substantial variability in incompatible element abundances. As $\mathrm{Na}_{2} \mathrm{O}$ decreases, all the incompatible elements also decrease substantially, even when all data are normalized to a constant and high $\mathrm{MgO}$ content.

The high alkali and $\mathrm{FeO}$ contents, conbined with the idea that ocean island volcanics are derived by low extents of melting from high pressures suggests that there is some additional factor influencing $\mathrm{FeO}$ and $\mathrm{Na}_{2} \mathrm{O}$ contents during partial melting that is not part of our melting calculations which means that it is not in the existing experimental data set. Possible candidates are certainly volatiles, which, because of their high solubility in magmas at high pressures, can play a particularly important role at low extents of melting at high pressures. A possible candidate for alkaline rocks is $\mathrm{CO}_{2}$. Although the detailed effects of $\mathrm{CO}_{2}$ on mantle melting are not well known, it is known that melting of $\mathrm{CO}_{2}$-rich peridotite results in more alkaline rocks. Indeed, the creation of highly alkaline rocks can probably only occur in the presence of appreciable $\mathrm{CO}_{2}$. The ultimate in alkalinity occurs in carbonatites [e.g., Wyllie, 1987; Nelson et al., 1988].

There is increasing evidence that $\mathrm{CO}_{2}$ is an important volatile constituent in the MORB source region as well. Dixon and Stolper [e.g., Dixon et al., 1988] have shown that the $\mathrm{CO}_{2}$ contents of erupted MORB are mostly controlled by the 


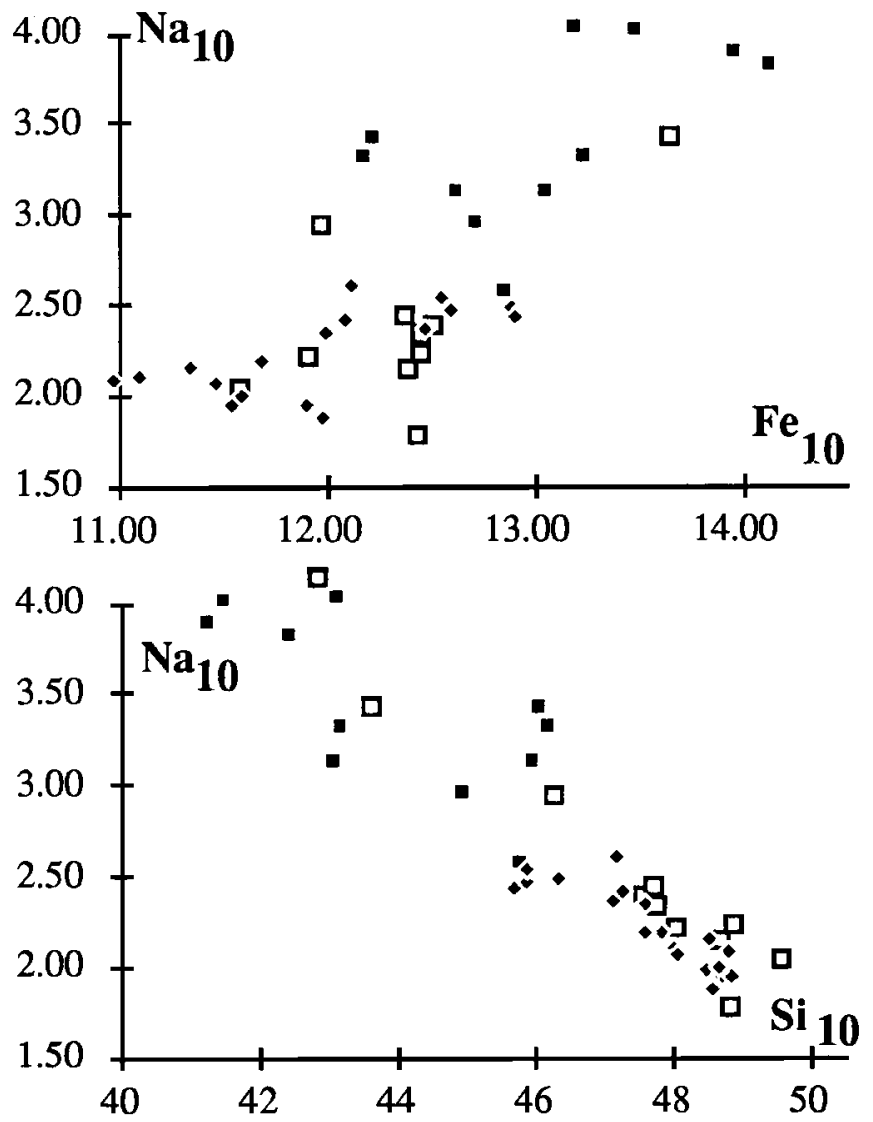

Fig. 65. $\mathrm{Na}_{10}$ vs. $\mathrm{Fe}_{10}$ and $\mathrm{Si}_{10}$ for basalts from Hawaii. $\mathrm{Na} \mathrm{a}_{10}$ is analogous to $\mathrm{Na}_{8}$, where $\mathrm{Na}_{10}$ is the $\mathrm{Na}$ content projected to $10 \%$ $\mathrm{MgO}$. Diamonds are samples from Mauna Kea [Frey et al., 1990]; small solid squares from the Honolulu volcanics [Clague and Frey, 1981]; open squares from Loihi seamount. Samples were screened to have $7.5-12 \% \mathrm{MgO},>41 \% \mathrm{SiO}_{2}$, and a sum of $>98 \%$ for the major element oxides. See Langmuir [1991] for more details.

solubility of $\mathrm{CO}_{2}$, which is very sensitive to pressure. The erupted $\mathrm{CO}_{2}$ contents simply reflect the pressure of degassing, and hence are minimum magmatic values, not indicative of the $\mathrm{CO}_{2}$ contents at high pressures or in the source regions. In addition, there are special samples known as "popping rocks" that are occasionally recovered from the sea floor and have exceptionally high $\mathrm{CO}_{2}$ contents. Sarda and Graham [1990] have suggested that the high inferred $\mathrm{CO}_{2}$ contents of these rocks are more indicative of the actual abundance of $\mathrm{CO}_{2}$ in MORB prior to degassing. Thus, it is possible that there is substantial $\mathrm{CO}_{2}$ in the MORB mantle source, and that, as for ocean islands, the deep parts of the melting column are alkaline rocks with low $\mathrm{SiO}_{2}$ and high $\mathrm{FeO}$. Then the melting column vector would have much higher $\mathrm{FeO}$ contents with high $\mathrm{Na}_{2} \mathrm{O}$ than we calculate based on the results of dry experiments. This might lead to melting column trends that are more similar to the slope observed for the Atlantic-type local vector. Whether $\mathrm{CO}_{2}$ is the explanation or not, it is clear that there is some aspect of the melting process that can create high $\mathrm{FeO}$ contents with high alkali contents, for we see this effect in almost every primitive alkaline rock.
Such an alkaline composition might be an appropriate endmember for the creation of the slow-spreading local vector, for it would contain high $\mathrm{Na}_{2} \mathrm{O}$, high $\mathrm{FeO}$, low $\mathrm{SiO}_{2}$, and high concentrations of $\mathrm{Sr}$ and other incompatible elements. Thus, it may provide a solution to the two criticalaspects of the slowspreading local vector that we have emphasized- the positive correlations of $\mathrm{Na}_{8,0}$ with $\mathrm{Fe}_{8.0}$ and $\mathrm{Sr}$.

\subsubsection{Local Variability and Ridge Segmentation}

The discussion of local variability above relates to the chemical systematics without reference to the spatial location of the samples within a particular ridge segment. However, the spatial distribution of MORB compositions has been a major focus of research, and ultimately detailed three-dimensional models of melt formation and segregation may be constrained by the along- and across-strike chemical systematics of enupted lavas. In addition, the spatial systematics of the local variations may help to guide our thinking concerning their origins.

Early work in the FAMOUS area [Bryan and Moore, 1977; variations across the rift valley over a limited distance along strike. Such across-strike variations now seem best interpreted as short-term temporal variability. This classic data set therefore now provides strong evidence for substantial temporal variability in the chemistry of eruptives over short time intervals (thousands of years). The amplitude and period of temporal variability, should ultimately provide very useful constraints on melting models, but this information is just beginning to be available [e.g., Hekinian and Walker, 1987; Reynolds et al., 1992], and cannot yet be used to constrain processes of melt formation and segregation.

There are sufficient data along strike, however, to begin to consider some of the spatial systematics of local chemical variability. For example, in the late 1970 s and early 1980 s attention focussed on chemical variations as a function of proximity to tectonic offsets along axis such as ridge offsets and propagating rifts [Hekinian and Thompson, 1976; Schilling and Sigurdsson, 1979; Natland and Melson, 1980; Christie and Sinton, 1981; Sinton et al., 1983; Bender et al., 1984; Langmuir and Bender, 1984]. In these and subsequent studies, evidence was found for local variations in the extent of melting and degree of fractionation. Bender et al. [1984], for example, noted chemical changes indicative of lower extents of melting adjacent to some transform faults, consistent with the prediction of such edge effects by Fox and coworkers [e.g., Fox and Gallo, 1984]. Subsequent work has shown that "magmatic edge effects" can occur even at some minute offsets of the East Pacific Rise [Langmuir et al., 1986; Sinton et al., 1991], but are not present at some of the largest offset transform faults [LeRoex et al., 1989; Klein et al., 1991; Reynolds and Langmuir, 1992 in press].

Recent work has also focussed on detailed sampling of entire ridge segments in order to explore the nature of along-strike chemical variability on the scale of a single spreading cell. In 
the Pacific, Langmuir et al. [1986] and Sinton et al. [1991] found substantial chemical variability over short distances, but also that several tectonic offsets often had significant chemical discontinuities across them, as first noted by Thompson et al. [1985]. In the Pacific, there is little variation in axial depth, gravity anomalies, and probably crustal thickness, and in the chemical data it does not currently appear that there are systematic chemical variations within offset-bounded ridge segments. Batiza et al. [1990] have suggested that regular variations across-axis in the Pacific can be studied using samples from seamounts.

For slow-spreading ridges, there have been many suggestions of a relationship between melt generation and position within a spreading segment [Whitehead et al., 1984; Lin et al., 1990]. Certainly, one can imagine a number of possible relationships between the melting regime and offsets of the ridge axis, and many of these relationships should have predictable petrological consequences. For example, preferential active upwelling in the centers of segments should lead to greater extents of melting at lower pressures, and greater crustal thicknesses. The combination of petrological data on carefully located samples with measurements of crustal thickness, geophysical properties such as gravity and theoretical modeling of melt segregation should lead to coherent and testable models that relate these various aspects of ocean crust formation.

Currently, however, there are few data that can be used to move in this promising direction, since combined detailed studies of ridge segments are just beginning. Batiza et al. [1988] sampled a $100 \mathrm{~km}$-long, transform-bounded segment of the southern MAR, and found a pattern of chemical variability that they suggested reflected spatial systematics in the melting regime beneath the ridge (Figure 66a). They found a complex along-axis pattern where samples from the shallow center of the ridge segment had chemical characteristics similar to those at the deep ends of the segment. Reynolds and Langmuir [1992, in revision] examined the ridge segment south of the Kane transform in the N. Atlantic, and found a rather different pattem (Figure 66b). It is intriguing, however, that over the same length scale, independent of the ridge offsets, the pattern of chemical variation looks rather similar. The data from these two regions (Figure 66) shows that we are still not able to generalize relationships between chemistry and spatial position within a ridge segment. This type of data, however, holds much future promise for elucidating both the origin of the slow spreading local variations, and the relationships among melt production, ridge tectonics and basalt chemistry. Systematic spatial relationships that relate basalt composition to physical characteristics of the ridge, such as spreading rate, transform spacing, axial depth, gravity anomalies or stage of a temporal cycle will provide a coherent data set that can be used to test quantitative models for ocean crust generation.

\subsection{The Nature of MORB Primary Magmas}

The results of the modeling and the discussion of the origins of the global and local compositional vectors cast a different light on the long-standing question of MORB primary magmas [e.g., O'Hara, 1968a; Elthon, 1989; Stolper, 1980; Fujii and Scarfe, 1985; Presnall and Hoover, 1984; Kinzler and Grove, $1992 a$ and $b ;$ Falloon and Green, 1987; Elthon, 1989]. The classical approach to this problem emphasized one or a small range in primary magmas, and that such magmas can be understood in terms of a pressure of segregation from the mantle. The correlation between pressure of melting and extent of melting, however, requires that melts of varying extents can be sampled from throughout the melting regime, as suggested by Langmuir et al. [1977], and that melts sampled at the surface result from pooling of diverse compositions from different pressures, as emphasized also by $O^{\prime}$ Hara [1985]. This means that it is more accurate to speak of mean pressures and extents of melting, and that the primary magmas that may arrive at the surface are not compositions that were themselves in equilibrium with the mantle at a single specified temperature and pressure. This complicates the classical approach to the primary magma problem of using multiple saturation experiments to test whether a particular composition is a primary magma in equilibrium with the mantle at some specific temperature and pressure. Melts at the surface are mean melts derived from ranges of $T$ and $P$.

Another important corollary is the likelihood of a wide diversity of primary magma compositions present beneath all ridge axes, since the melting regime contains a variety of melt compositions within it. The variety is produced both by the different extents and pressures of melting within the melting regime, and as a result of melting a mantle that is heterogeneous on a range of scales. Therefore, there are multiple MORB primary magmas, and the challenge is to understand the systematic differences rather than to identify which of them is the true primary magma.

The primary magmas can also have a rather wide range of $\mathrm{MgO}$ contents. The exact value depends in part on the melting process - fractional melting causes significantly higher $\mathrm{MgO}$ contents than does equilibrium melting. $\mathrm{MgO}$ contents are controlled primarily by the pressure of intersection of the solidus. Mean melts from hot spot regions where melting begins at greater depth may have greater than $15 \% \mathrm{MgO}$, and hence be picritic. The deepest ridges over the coldest mantle may produce magmas with as little as $10 \% \mathrm{MgO}$. Thus proponents of both high and low $\mathrm{MgO}$ primary magmas were partially correct, for both exist in different regions. Average ocean ridge basalt, however, is now quite well constrained to be generated by an intersection of the solidus at about $20 \mathrm{kbar}$, a mean extent of melting of about $10 \%$, at mean pressures of about $10 \mathrm{kbar}$, and to contain approximately $12 \mathrm{wt} \% \mathrm{MgO}$.

\subsection{Interpretation of MORB Major Element Chemistry}

We have now observed how major elements vary on many ocean ridges, and considered a number of theoretical approaches to account for these observations. If the working model applied generally, and both global and local trends corresponded with the calculated variations in mantle temperature and variations within the melting regime, 

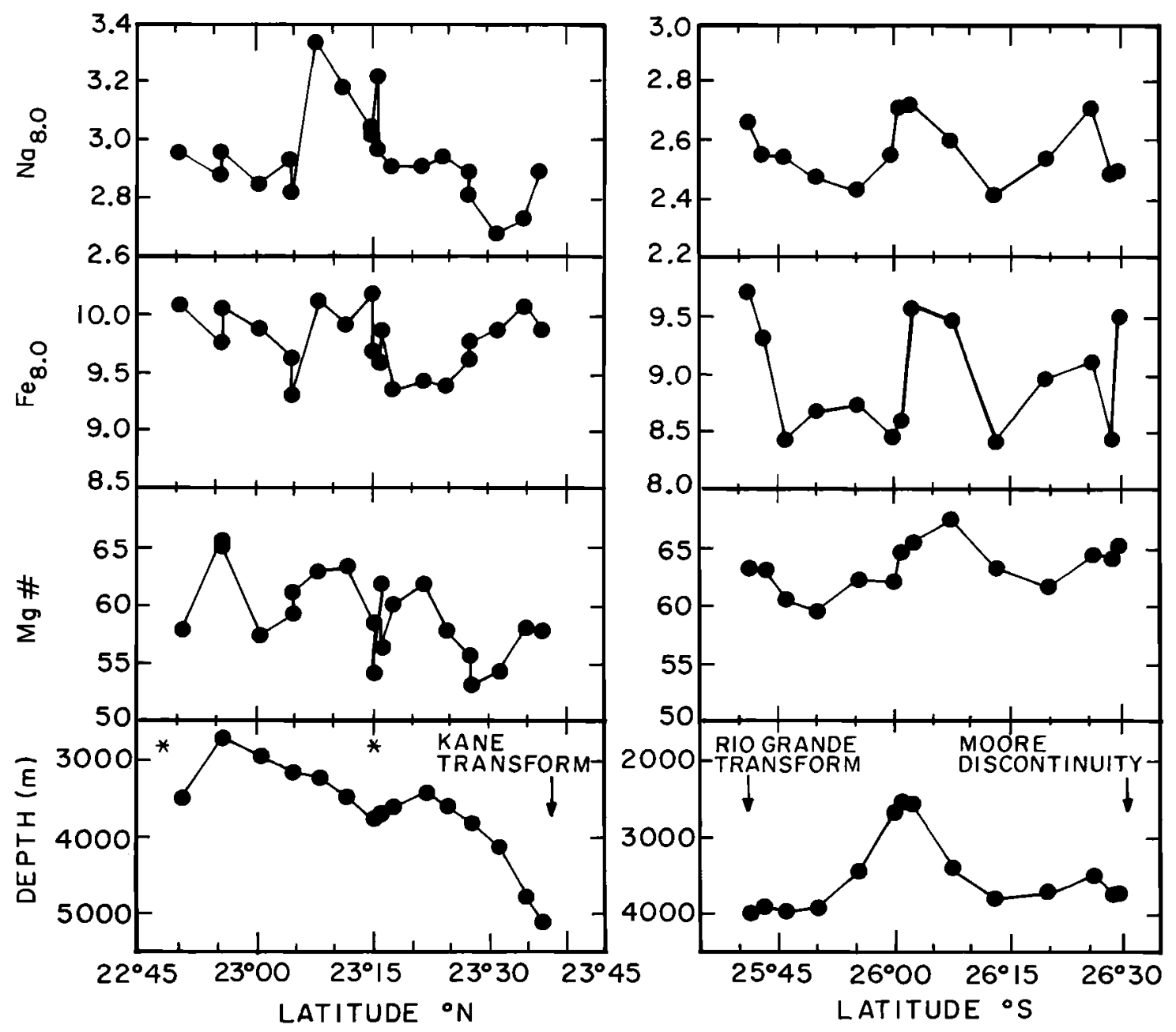

Fig. 66. Comparison of data for ridge segments along the Mid-Atlantic Ridge, one from south of the Kane Transform in the N. Atlantic [Reynolds and Langmuir, 1992], and the other from south of the Rio Grande transform in the S. Atlantic [Batiza et al., 1988]. The S. Atlantic data, taken from a figure presented by Batiza et al. [1988], were not corrected for interlaboratory bias. The data points are for samples collected along strike of the ridge, with an effort made to sample the neovolcanic zone where possible. Thus, the patterns reflect as closely as possible the instantaneous variation in chemistry along a ridge segment. For each side of the figure, the scales are adjusted so both horizontal and vertical scales are comparable. The S. Atlantic data are for a single ridge segment. The N. Atlantic data span two ridge segments, separated by small non-transform discontinuities whose position is indicated by asterisks.

respectively, then interpretation of MORB data would be simple. We could write equations for mantle temperature and extent of melting that could be used for chemical analyses from any particular ridge segment. The $\mathrm{Na}_{2} \mathrm{O}$ and $\mathrm{FeO}$ contents of individual basalts would specify both the mantle temperature and the depth within the melting regime from which that particular basalt was derived. This approach has been utilized recently by Niu and Batiza [1991].

But the situation is not so straightforward. There is structure within the global correlations caused by hot spots and by subtle differences between ocean basins. And there are distinct local trends on the MAR and EPR, neither one of which corresponds with the melting calculations in their present state of advancement. How, then, at this stage of our understanding, can we interpret a particular data set? For the East Pacific Rise, it is our current view that most of the local variations are caused by small scale heterogeneities. Hence variations in $\mathrm{Na}_{8.0}$ along strike are not directly indicative of variations in mantle temperature and may not even indicate extent of melting. Instead, there may be information concerning the nature of melting and melt segregation from a heterogeneous mantle but the content of this information remains to be elucidated. For slow-spreading ridges, although we do not have a quantitative explanation for the local trend, it is at least clear that it is caused by melting processes. Therefore, even though we cannot yet quantitatively reproduce it, we interpret the local trends provisionally as indicative of position within a melting regime - possibly with additional complications of small scale temperature variations associated with segmentation.

If we accept these provisional interpretations of the slowspreading local trends, then the intersection of these trends with the global correlations gives the regional mantle 
temperature. This is the concept behind the $\mathrm{Fe}_{8,0}^{\mathrm{G}}$ parameter of $K L 89$, a parameter which simply indicates the $\mathrm{Fe}_{8.0}$ contents at which a local trend intersects the global array. $\mathrm{Fe}_{8.0}^{\mathrm{G}}$ tracks variations in regional depth on slow-spreading ridges very well. The use of this parameter is still open to question, however, because we do not yet have the theory that adequately accounts for the slow-spreading local trends. Use of this parameter on the EPR is not appropriate, since the local variations there have a different cause. The best estimate of $\mathrm{Fe}_{8.0}^{\mathrm{G}}$ will be to have a suite of data from a particular ridge segment, and to determine where those data intersect the global correlations. Then the new melting radel allows us to calibrate $\mathrm{Fe}_{8.0}^{\mathrm{G}}$ to temperature. First we need an equation for the global correlations for normal ridges, which is:

$$
\mathrm{Na}_{8.0}=-0.36 \mathrm{Fe}_{8.0}+6.26
$$

$\mathrm{Fe}_{8.0}^{\mathrm{G}}$ is the intersection of the local trend with this global correlation line. (Note that the specific equations for $\mathrm{Fe}_{8.0}^{\mathrm{G}}$ from $K L 89$ do not apply here, because they were based on Smithsonian data, while the data in this paper and the calculations are based on Lamont data. $\mathrm{Fe}_{8.0}^{\mathbf{G}}$ should be determined for each data set). Then we have the following relationship between $\mathrm{P}_{\mathrm{o}}$ and $\mathrm{Fe}_{8.0}$, determined for the fractional melting model, which turns out to be almost linear:

$$
P_{0}=6.11 \mathrm{Fe}_{8.0}^{\mathrm{G}}-34.5
$$

$P_{0}$ then gives the temperature of intersection of the solidus $\left(1150+13 * P_{o}\right)$. Application of these relationships is uncertain near hot spots, because we do not yet know the cause of the offset of many hot spot points frcm the $\mathrm{Na}_{8.0}-\mathrm{Fe}_{8.0}$ relationships for normal ridges.

\section{Trace ElemENT Constraints}

Our approach thus far has emphasized the major element compositions of MORB. Approaching melting through major elements, however, is a recent developrrent. The common approach for many years was instead to approach melting problems through the trace element compositions of the samples [e.g. Schilling, 1975; White and Bryan, 1977; Langmuir et al., 1977; Perfit et al., 1983; Clague et al., 1981], and to use the major elements mostly as a sample screen. Trace elements appear to be an ideal tool to investigate melting processes, because the ratio of two moderately incompatible trace elements should change little during crystallization, but markedly during melting [Gast, 1968]. in practice, however, incompatible trace elements often vary more than one would predict, and these variations have not yet been shown to have straightforward relationships with the major element variations just presented. On the other hand, moderately incompatible and compatible elements do seem to show similar systematics to the major elements. In this discussion, we focus on the contrasting global and local systematics of trace element abundances in MORB, and also on the important distinction between the highly incompatible elements as a group, and the other less incompatible trace elements.

\subsection{Global Variability of Trace Elements}

The major element model for the global correlations carries with it specific predictions for the abundances of trace elements, if the source were homogeneous. The source is likely to be quite homogeneous for moderately incompatible elements [e.g., Hoffmann, 1988], and indeed, for samples far from hot spots, some compatible and moderately incompatible trace elements correlate well with $\mathrm{Na}_{8.0}$ in a way hat can be predicted by melting. For example, $\mathrm{Sc}$ behaves similarly to $\mathrm{CaO} / \mathrm{Al}_{2} \mathrm{O}_{3}$, and forms an inverse trend with $\mathrm{Na}_{8.0}$ for regionally averaged samples (Figure $67 a ; K L 87$ ). $K L 87$ also showed that $\mathrm{Sm} / \mathrm{Yb}$ correlates positively with $\mathrm{Na}_{8.0}$, and that $\mathrm{Ni}$ also shows systematic relationships with $\mathrm{Na}_{8.0}$ when the effects of olivine fractionation have been taken into account. Therefore for samples not dominated by hot spot effects, the global trace element variations are generally consistent with the inferences from the major element global vector, and hence with different extents of melting of the mantle on a regional basis.

As one examines progressively more incompatible elements, the relationships become more scattered. Figure $67 b$ shows averages of $\mathrm{Na}_{8.0}$ and $\mathrm{Ce}_{8.0}$, (see equation 16) for the calculation of $\mathrm{Ce}_{8.0}$, for the same regions as on the Sc plot. There is still a reasonable correlation which corresponds with melting models, but some regions have $\mathrm{Ce}_{8.0}$ contents that are slightly high for a given $\mathrm{Na}_{\mathbf{8 . 0}}$. The EPR, for example, is higher than regions with comparable $\mathrm{Na}_{8.0}$ from the MidAtlantic Ridge. The lower bound of the data, however, corresponds well with a melting model based on the major elements. Plank and Langmuir [1992, hereafter PL92] have called this line, which serves as a us ful reference, the "baseline". Once one moves to elements more incompatible than $\mathrm{Ce}$, the data become very scattered. For example, there is no simple relationship, between $\mathrm{Na}_{8.0}$ and $\mathrm{Ba}_{8.0}$, a highly incompatible element (Figure 67). Thus, coherence with major element global variations seems to be a function of element incompatibility. Highly incompatible elements seem to vary independently of the major elements. Of course, it is for just these elements that mantle heteroegeneity becomes increasingly important, as is well known. Note that the relationships shown in Figure 67 are for MORB samples far from hot spots. Including the samples near hot spots only increases the scatter on these diagrams.

Although hot spots are not part of any global correlations for trace element abundances, there are important spatial relationships associated with hot spots near ridges. These relationships have been extensively documented by Schilling and coworkers over the last twenty years [e.g., Schilling, 1973; Schilling, 1975; Schilling et al., 1976; Schilling et al., 1983]. Figure 68 shows the ratio of tivs light rare earth elements (REE), La and Sm, plotted agai-st latitude and depth for the northern Mid-Atlantic Ridge axis. There is an obvious relationship between the occurrence of the hot spots (e.g., Azores, Iceland, and Jan Mayen), shallow ridge axes and higher $\mathrm{La} / \mathrm{Sm}$ ratios. These observations led Schilling [e.g., 1972] to postulate mixing between a deep, incompatible element enriched plume source, and a shallow, depleted "normal" ocean 


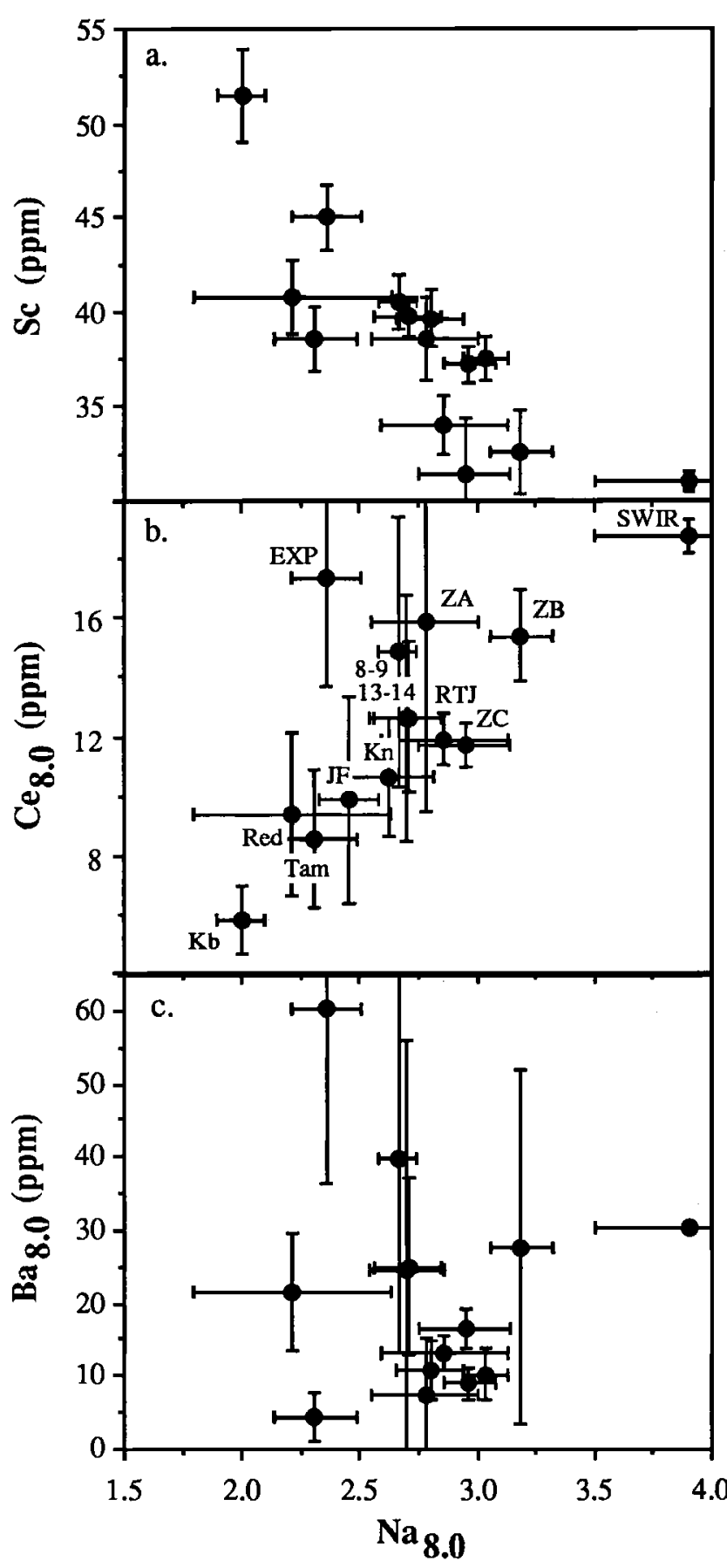

Fig. 67. $\mathrm{Na}_{8.0}$ vs. a) $\mathrm{Sc}$, b) $\mathrm{Ce}_{8.0}$, c) $\mathrm{Ba}_{8.0}$ for regionally averaged data. Each point is the average of samples from an individual region, and the error bars are one standard deviation of the valies. $\mathrm{Ce}_{8.0}$ and $\mathrm{Ba}_{8.0}$ calculated using equation given in the text. Regions are: Kolbeinsey Ridge (Kb) $17^{\circ} \mathrm{N}$ in the Red Sea (Red), near Tamayo transform of the EPR (Tam), Juan Fernandez microplate (JF), near Kane of the MAR (Kn), 13-14 ${ }^{\circ} \mathrm{N}$ on EPR (13-14), 8-9 $\mathrm{N}$ on EPR (8-9), south Explorer Ridge (Exp), Zones A, B and C of the Australian Antarctic Discordance (ZA, ZB, ZC), near the Rodriguez or Indian Ocean Triple Junction (RTJ), and around $30^{\circ} \mathrm{S}$ on the Southwest Indian Ridge (SWIR). For references, see Plank and Langmuir [1992]. ridge basalt source. Although some workers have questioned the depths in the mantle of the differing source compositions, it is nevertheless clear that a dominant signal in incompatible trace element variability is mantle heterogeneity associated with hot spots, a conclusion also indisputably supported by radiogenic isotope data [e.g., Zindler and Hart, 1986].

Observations regarding the width of the chemical anomalies as well as the different chemical signatures associated with different plumes have led to complex and quite detailed models for plume/ridge interactions [e.g., Schilling, 1985; Hanan et al., 1986]. Schilling [1985], for example, has shown a correlation between the width of the chemical anomaly on the ridge, and the distance between the plume and the ridge. Thus, the sharp spikes in La/Sm that occur at latitudes $71^{\circ} \mathrm{N}$ and $35^{\circ} \mathrm{N}$ might be attributed to plumes that are presently far from the ridge axis. Although Schilling's models explain a large number of observations remarkably well, there are aspects of the long wavelength signal that are difficult to understand. For example, the gradients around both Iceland and the Azores are highly asymmetric. There are also spikes in incompatible element abundances at $43^{\circ} \mathrm{N}$ and $45^{\circ} \mathrm{N}$ along the Mid-Atlantic Ridge that are not currently well explained by off-axis hot spots.

It is important to note as well that the relationships for hot spots between incompatible element enrichment and depth are

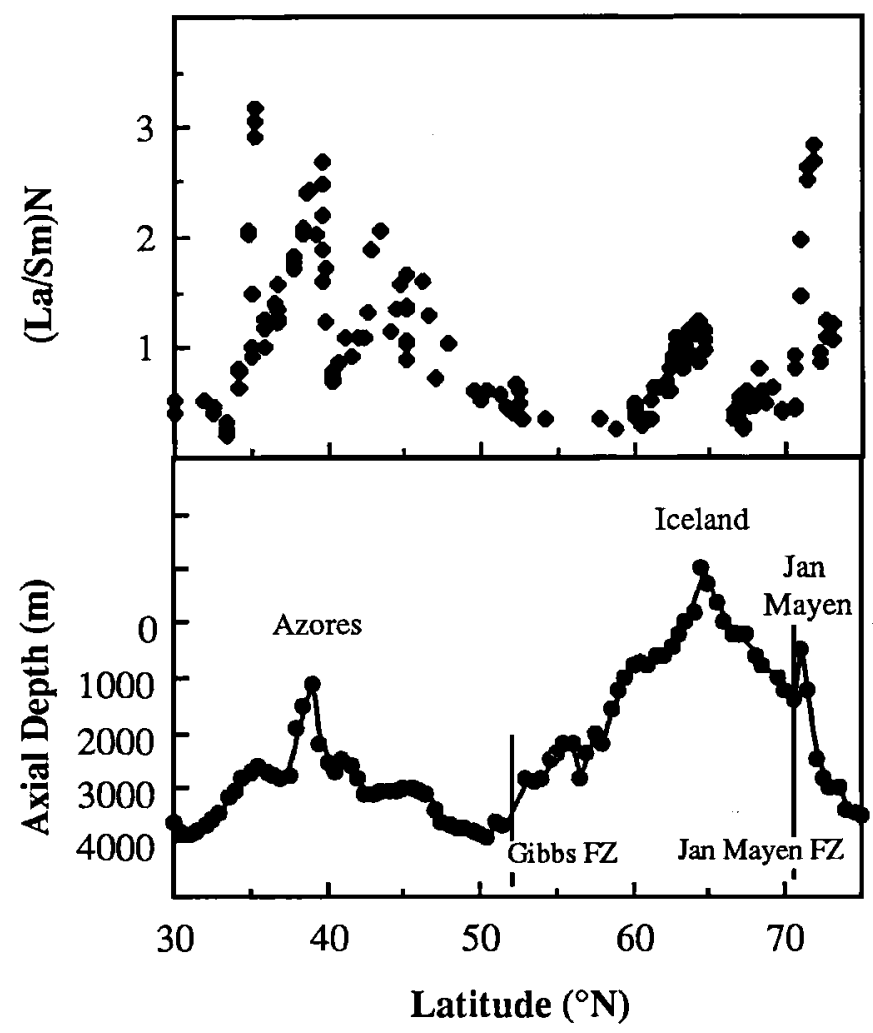

Fig. 68. Latitude ( $\left.{ }^{\circ} \mathrm{N}\right)$ vs. smoothed axial depth and (La/Sm)N for the northem Mid-Atlantic Ridge. Latitudinal depth variations, taken at $5^{\circ}$ intervals, are from LeDouaran and Francheteau [1981] and Vogt [1986]; La/Sm data are from Schilling et al. [1983]. 
not "global," because the magnitude of incompatible element enrichment does not diminish systematically from the shallowest to the deepest ridge segments. This is apparent from Figure 68, where it can be seen that Iceland is a much shallower feature than the Azores, and yet has a substantially lower $\mathrm{La} / \mathrm{Sm}$ ratio. Thus in trace elements as well as major elements and depth, the Azores and Iceland are very different hot spots.

These diverse aspects of the trace element data can be understood in part as the result of two contrasting effects. Some of the deepest parts of the ocean ridge system, such as the MidCayman Rise [Thompson et al., 1979] and Australian Antarctic Discordance [Klein et al., 1991] are moderately enriched in incompatible elements compared to shallower ridges because of the lower extents of melting that gave rise to them. Hotter temperatures for normal mantle cause greater extents of melting and relative depletion in incompatibles. But the hottest regions, usually associated with hot spots, have a large trace element excess associated with mantle heterogeneity. Therefore both the deepest and shallowest ocean ridges are often more enriched in incompatible elements than normal depleted ridges that lie at intermediate depths. The contrasting trace element behavior leads to some of the great chemical boundaries in the oceans, such as the Hiayes Fracture Zone, where mantle enriched by the Azores province is bounded on the northern side of the fracture zone, while relatively hot normal MORB mantle lies on the southern side [Bougault and Treuil, 1980]. The temperature may change progressively across the fracture zone, but the trace eleninents change abruptly because of the hot spot trace element influence on one side and not on the other.

Another important aspect of the global variability of trace elements is the contrast between the EPR and MAR. The EPR has a single hot spot, associated with Easter Island, that has a very limited effect on depth and chemistry. In contrast, much of the depth and chemistry of the Atlantic are strongly influenced by hot spots. The EPR, however, does seem to have some long wavelength gradients in trace element chemistry not obviously associated with hot spots. Sinton et al. [1991] have shown that there is a smooth gradient in incompatible element enrichment along several hundred kilometers of the southern EPR. The EPR also has apparently random occurrences of enriched MORB, in contrast to the highly localized occurrences associated with hot spots on the MAR. Thus the MAR and EPR, and possibly fast- and slow-spreading ridges in general, appear to have a rather different relationship with hot spot activity.

\subsection{Local Variability in Trace Elements}

Substantial variations in trace element abundances and ratios are well established for many small-scale regions at a variety of spreading rates [e.g., White and Bryan, 1977; Langmuir et al., 1977; Hekinian and Walker, 1987; Shibata et al., 1979; Thompson et al., 1985; Batiza and Vanko, 1984; Michael et al., 1989; Karsten et al., 1990]. In some cases, the variability appears to be associated with the segmentation of the ridge [e.g., Bender et al., 1984], while in others there are simply substantial differences among closely spaced samples. The trace element variations are often interpreted as evidence for different extents of melting of the mantle or for source heterogeneity, but these interpretations are seldom connected to the major element data.

The local systematics observed in the major elements provide a new perspective from which to view the trace element variations. We now can compare trace element variations, which have often been interpreted by quite specific partial melting models, with major element variations that are also considered to provide information concerning the melting process. We have seen above that there is a useful "baseline" based on regional averages that relates major and trace element compositions. From some trace elements, there is also evidence for pooling of individual melts from throughout the melting regime. For example, Salters ana Hart [1989] found a relationship between $\mathrm{Lu} / \mathrm{Hf}$ ratios and $\mathrm{Hf}$ isotopes that has implications similar to those that can be inferred from the relationship between $\mathrm{Na}_{8.0}$ and $\mathrm{Fe}_{8.0}$ - that melts are pooled from throughout the melting regime. The Lu-Hf relationships support the inference from major elements that there is a local vector that reflects processes within the melting regime.

Upon examining individual samples from any specific region in detail, however, an importent contrast emerges between the major and trace element models - the trace element models almost always suggest a smaller inferred extent of melting than we have found for the major elements. For example, Bender et al. [1984] found evidence for extents of melting of around 5\% for the Tamayo Region on the EPR, yet evidence from major elements and crustal thickness suggests instead a mean extent of melting of $10 \%$ or more. Therefore we see an overall consistency between avcrage trace element composition and major element models fur samples far from hot spots, but models based on separate analyses from an individual region lead to contradictory results.

The source of this contrast between major and trace element models can be seen in an examination of the unaveraged data. Although the averaged $\mathrm{Ce}_{8.0}$ data for samples far from hot spots showed a reasonable correlation with $\mathrm{Na}_{8.0}$, examination of the unaveraged data shows a large degree of scatter (Figure 69a). When individual samples are identified separately (Figure 69b), the data often form steep yet well-defined trends, where $\mathrm{Ce}_{8.0}$ may vary by a factor of three or more, while $\mathrm{Na}_{8.0}$ varies by only 20\% [PL92]. These steep trends, for example, are apparent in the data from the Red Sea near $17^{\circ} \mathrm{N}$, the EPR near $9^{\circ} \mathrm{N}$, and the FAMOUS area. It is these steep trends that require small extents of melting for models based on trace elements. On the $\mathrm{Ce}_{8.0}-\mathrm{Na}_{8.0}$ diagram (Figure 69 ), the melting curve has a steep slope only at small extents of melting, because only when $F$ is small can there be significant separation between elements with small D's. Therefore attempting to fit a steep trend with a trace element model, one is driven to small F's to arrive at the necessary fractionation among incompatible elements.

These steep trends provide another view of the long-standing observation in MORB studies of a "trace element excess" that 

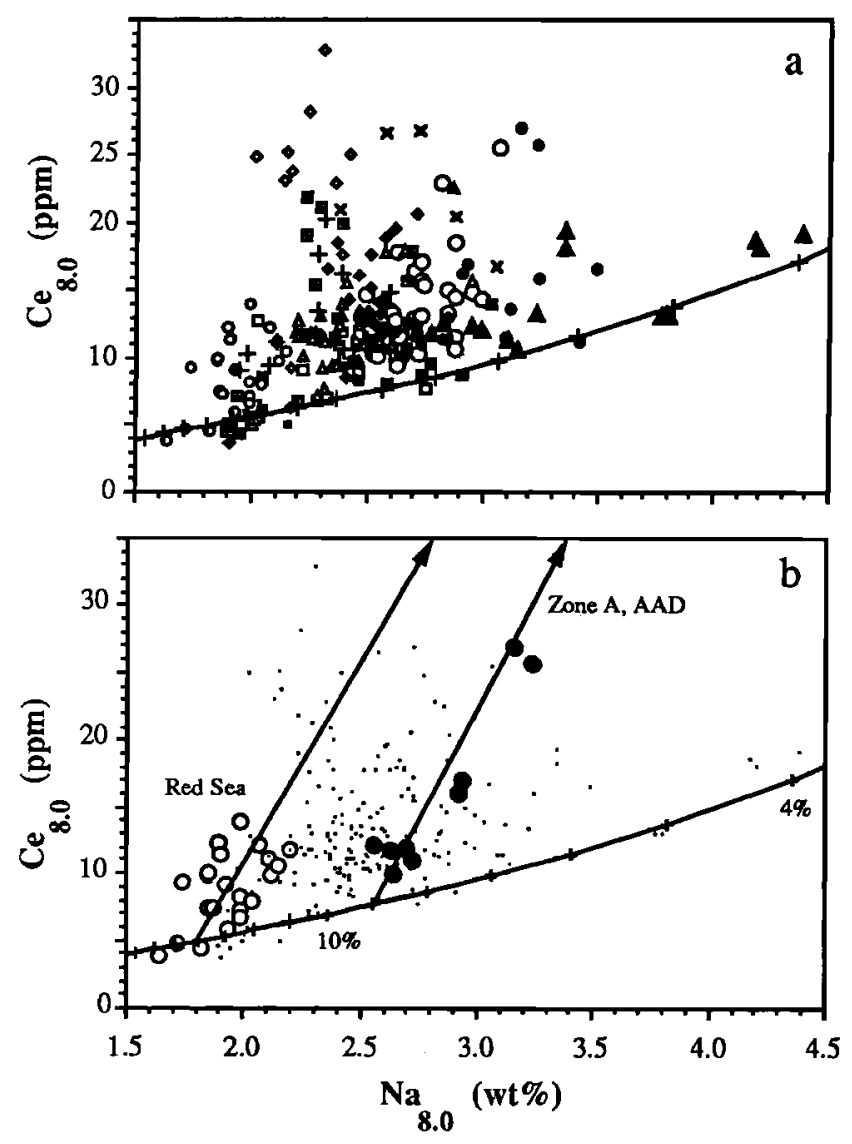

Fig. 69. $\mathrm{Na}_{8.0}$ vs. $\mathrm{Ce}_{8.0}$ for MORB, with a calculated melting "baseline". a) Basalt data points for individual samples (same as in Figure 25). Melting curve is fit through the bottom of the data array for an equilibrium melting model, where $\mathrm{C}_{0}$ for $\mathrm{Na}_{2} \mathrm{O}=0.3 \mathrm{wt} \%, \mathrm{C}_{0}$ for for an equilibrium melting model, where $C_{0}$ for $\mathrm{Na}_{2} \mathrm{O}=0.3 \mathrm{wt} \%, \mathrm{C}_{0}$ for
$\mathrm{Ce}=0.7 \mathrm{ppm}, \mathrm{D}_{\mathrm{C}_{e}}=.001$ and $\mathrm{D}_{\mathrm{Na2O}}=0.03$. Extent of melting $(4-17 \%)$ indicated as tick marks along the melting curve. b) Steep trends shown by the data from the Red Sea and Zone $A$ of the AAD, and mixing lines between melts on the baseline and a $0.2 \%$ melt of the same mantle source. Figure from Plank and Langmuir [1992].

is present after correction for low pressure differentiation. When least squares calculations have been carried out to evaluate low pressure fractionation, the incompatible trace elements often show too much variation. These variations have been interpreted in terms of complex magma chamber processes [e.g., Bryan and Moore, 1977; Dungan and Rhodes, 1978; Hekinian and Walker, 1987] or as manifestations of distinct parent magmas derived by different extents of melting of the mantle [e.g., White and Bryan, 1977; Langmuir et al., 1977]. This latter argument was based on studies of high $\mathrm{MgO}$ samples that could not have undergone substantially different amounts of fractionation. The steep $\mathrm{Na}_{8 .}, \mathrm{Ce}_{8.0}$ correlations create difficulties, however, even for a partial melting explanation, for the variations in trace e'ement ratios exceed those predicted by major element melting models.

Another aspect of trace element data that poses difficulties for melting models is the Th-U-Ra disequilbria studies that are beginning to appear [e.g. McKenzie, 1785a; Williams and
Gill, 1989; Goldstein et al., 1989; 1991; Reinitz and Turekian, 1989; Rubin and Macdougall, 1988; 1990]. Taken at face value, these new data would seem to require very low extents of melting beneath ridges in order to fractionate these highly incompatible elements from one another during melting. However, the $\mathrm{Ra}$ excesses with respect to $\mathrm{Th}$ are so large that the melt fractions required are smaller than seems physically reasonable. In addition, new experimental data [Latourette and Burnett, 1992] show that $U$ is more incompatible than $T h$ in clinopyroxene, which suggests that equilibrium melting processes will not fractionate the elemerts from one another in the sense that is observed. These new data are particularly puzzling and very important, for the fractionations of the $U$ series disequibria must take place on very short time scales $\left(10^{3-} 10^{5} \mathrm{yrs}\right)$ prior to eruption. It appears that some process other than equilibrium melting will be reçuired to account for the data.

The trace element data thus clearly provide another indication of our incomplete understanding of the melting process. The bright side is that local variability of trace elements potentially contains much information concerning the melting process that we do not receive from major elements alone.

One idea that might aid in explaining this effect was raised by $O^{\prime} H a r a$ [1985], who suggested that trace element abundances and ratios are influenced by the shape of the melting regime. If true, then the trace element signature could be used to constrain the shape, and hence the mixing function, that is applicable to ridges under different conditions. We therefore need to consider from a theoretical point of view what the local trace element variations may be revealing concerning the nature of the melting regime beneath the ridge. We can then return to potential interpretations of the trace element data.

\subsection{Trace Elements and Melting Regime Shape}

O'Hara [1985] suggested that incompatible elements may be differentially enriched by pooling melts from melting regimes of complex shapes. PL92 argue that this conclusion is based on a misunderstanding of the difference between the $\bar{F}$ of the melting regime, as defined in section 4.3.1, and the maximum $F$ at the top of the central column of the melting regime. This misunderstanding has propagated in the literature, with many papers comparing the results of pooled melt calculations with batch melting to the maximum $F$ of the melting regime rather than the $\bar{F}$ of the melting regime.

The maximum F, of course, is applicable only to the top of the central column of the melting regime, where the extent of melting is greatest and the incompatible elements most diluted. The $\overline{\mathrm{F}}$ refers to the melts pooled from throughout the melting regime, and it is this $\overline{\mathrm{F}}$ that is the appropriate reference point for trace elements as well as major elements. The $\overline{\mathbf{F}}$, as is clear from the residual melting column concept, is simply the total amount of melt divided by the total volume that has melted. A perfectly incompatible element will be exclusively in the liquid, and its concentration will be simply $C_{0} / F$. This is 
identical to the concentration produced by batch melting to the same extent. In fact, the $\overline{\mathbf{F}}$ may always be calculated from the perfectly incompatible element $(D=0)$, which will increase with $C_{o} / F$, regardless of the mixing or melting functions involved. It is not identical for elements that are not perfectly incompatible, but the discrepancies with batch melting are small.

This is illustrated in Figure 70a, where melts up to $5 \%$ are pooled, with a mean extent of melting of $3 \%$. The solid line shows the compositions of the individual melts, and the triangle the composition of the pooled melt, which lies exactly on the line calculated by the simple model. Figure $70 \mathrm{~b}$ shows
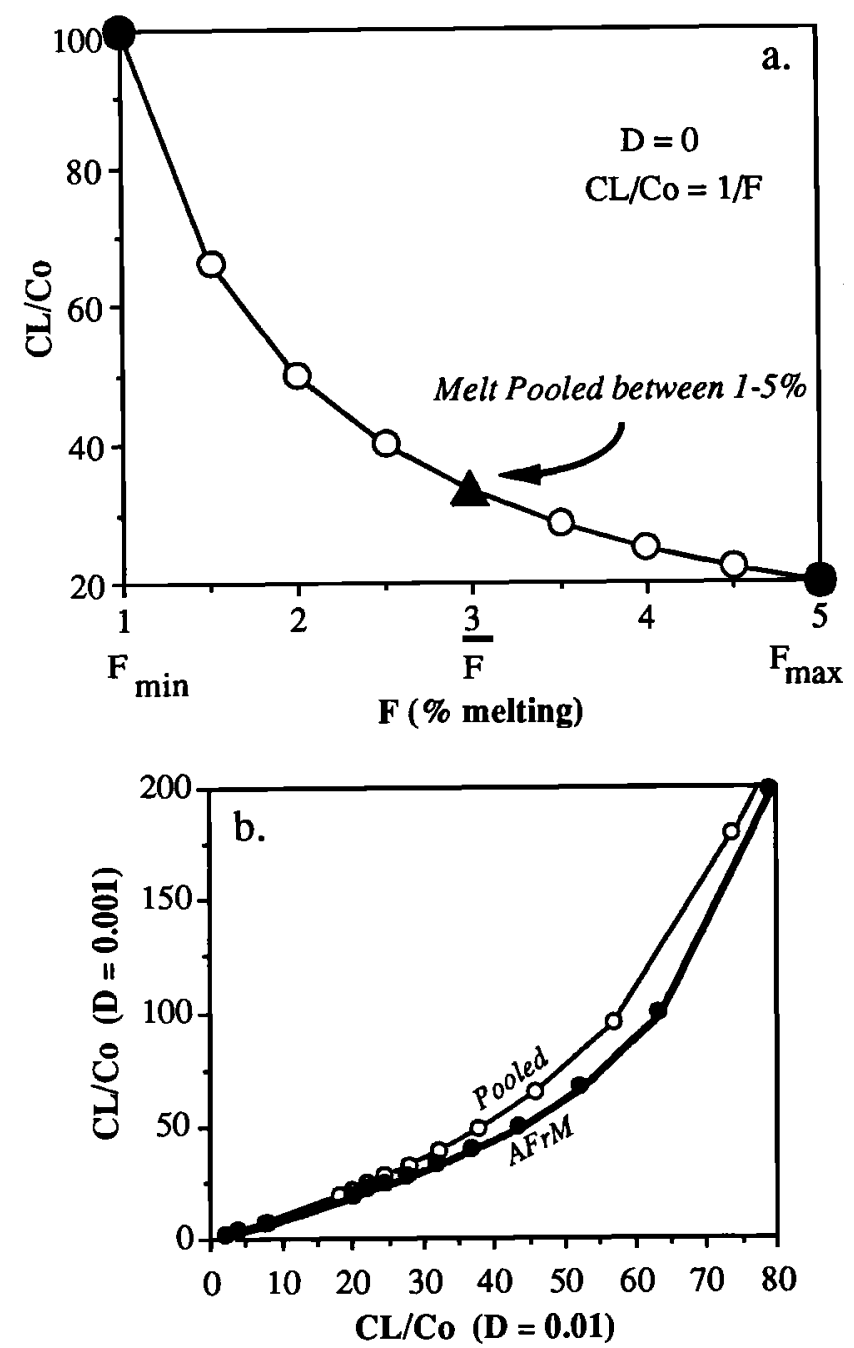

Fig. 70. Comparison of the chemical consequences of pooling melts vs. a simple batch melt to the same mean extent. a) Curve is concentration of a perfectly incompatible element vs. extent of melting $(F)$ for equilibrium melting between 1 and $5 \%$. Large triangle is mixture of melts pooled between 1 and $5 \%$, which lies on the melting curve itself at the same mean $\overline{\mathrm{F}}, b$ ) The concentration of two incompatible elements $(D=0.001$ and 0.01$)$ for simple accumulated fractional melting (AFM) and pooled AF melts for the same mean $\vec{F}$ using the equations given in Plank and Langmuir [1992]. the results of the same calculation for two elements whose D's differ by a factor of ten. In this case the pooled melt differs very slightly from the batch melting curve, but the differences in fact are indistinguishable, if one considers simply the analytical and geological uncertainties in any chemical analysis. The same result applies for a very wide range of shape models, so long as the melting function is continuous, and melts are mixed in proportion to F. All reasonable shapes for melting regimes beneath ridges reduce to this kind of pooling, so the shape of the melting regime has little effect on the composition of the pooled melt, in comparison to a batch melt to the same $\overline{\mathrm{F}}$. Of course, as was the case with $\mathrm{Fe}$, this conclusion does not apply for elements that show a strong pressure dependence for their distribution coefficients.

This result can be understood intuitively from Figure $70 b$. Mixing produces straight lines on such a diagram. If the melting function is continuous, there are compositions in the melting regime all along the batch melting curve, so there is a mixing end-member all along the curve. Mixtures of all the points along the curve never lead to a composition that deviates significantly from the curve.

These considerations show that the shapes considered by O'Hara [1985] do not produce trace element variations that are significantly different from batch melting to the same $\overline{\mathrm{F}}$. The positive aspect is that simple trace element models can be accurate despite a lack of knowledge of the details of the shape. The negative aspect is that trace elements also then provide no constraints on the shape.

These results also have important implications for recent "inverse" models of melting beneath ridges proposed by McKenzie and O'Nions [1991]. They attempt to invert the distribution of melt in the melting regime from rare earth element analyses, using a single averaged analysis of the REE to arrive at a solution. Their $\mathrm{K}_{\mathrm{d}}$ 's and source are set $a$ priori. Because most MORB are derived by melting from within the spinel peridotite stability field, the appropriate models to evaluate the results of McKenzie and O'Nions [1991] are those of constant $K_{d} s$, and variable extents of melting. In this case, a batch melt of the mean extent of melting within the melting regime will be essentially indistinguishable from a mixture of melts from throughout the melting regime. The surprising implication is that NO unique solution of melt distribution can be obtained from the REE average, for any volume of melt from any depth within the spinel stability field that has melted to that mean extent will yield the same REE pattern.

These simple considerations also show what process could produce a result that differs substantially from batch melting [PL92]. If mixing took place between two distal portions of the curve in Figure $70 b$, with no intermediate compositions participating, then mixtures would lie along a mixing line that could deviate substantially from the batch melting curve. This situation might be applicable to ridges, for the presence of small amounts of volatiles in the mantle source causes there to be a deep, volatile saturated solidus far below the dry solidus that we have used in our melting models, as illustrated in Figure 71 , and emphasized previously by McKenzie [1985b] and Galer and O'Nions [1986]. In this region between the volatile- 

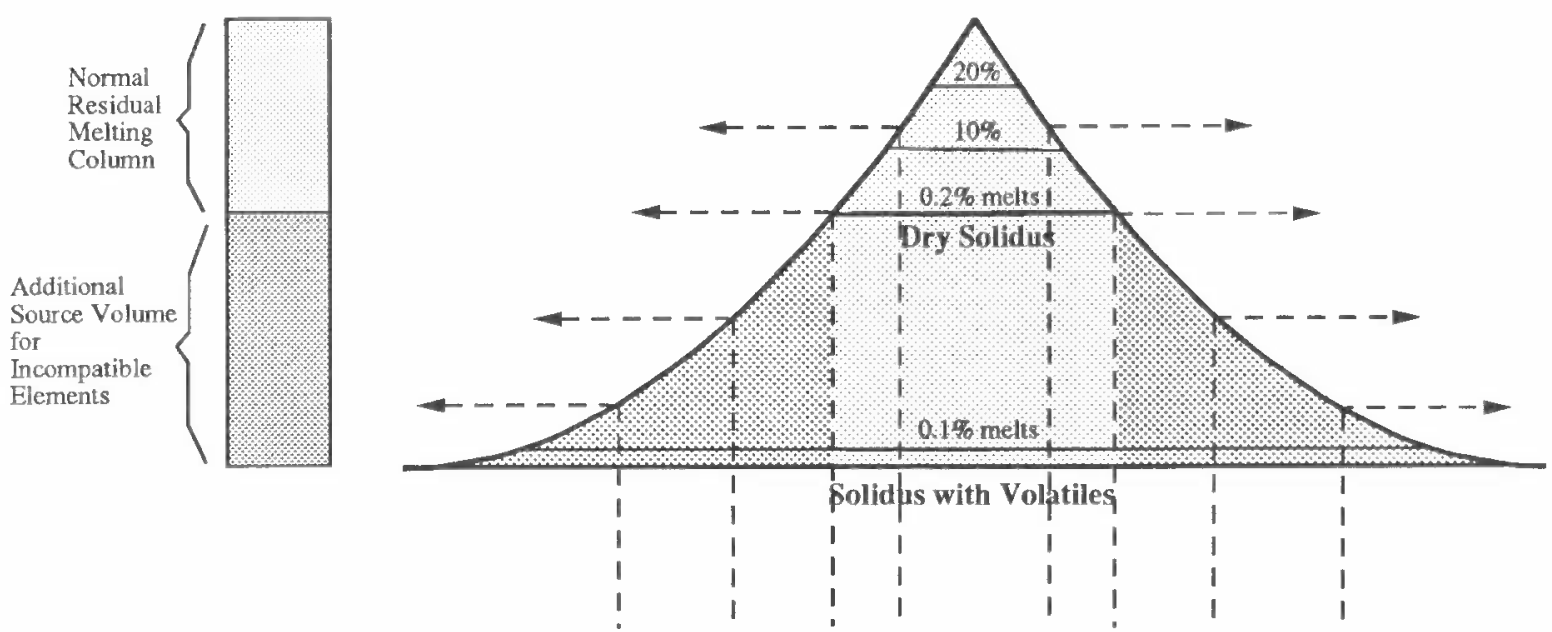

Fig. 70. Comparison of the chemical consequences of pooling melts vs. a simple batch melt to the same mean extent. a) Curve is concentration of a perfectly incompatible element vs. extent of melting (F) for equilibrium melting between 1 and $5 \%$. Large triangle is mixture of melts pooled between 1 and $5 \%$, which lies on the melting curve itself at the same mean $\overline{\mathrm{F}}, b)$ The concentration of two incompatible elements $(\mathrm{D}=0.001$ and 0.01$)$ for simple accumulated fractional melting (AFM) and pooled AF melts for the same moun $\bar{F}$ using the equations given in Plank and Langmuir [1992].

saturated and dry solidi, the extent of melting will be very small, controlled by the small amount of volatiles contained within the mantle. These volatile-rich melts will be very low in viscosity and density, enabling them to segregate and contribute to the large volume of melt prctuced above the "dry solidus." These low degree melts would have high levels of enrichment of the most incompatible elements and could produce the steep $\mathrm{Na}_{\mathbf{8}, 0}$ - incompatible element trends mentioned above. The extra low degree melts would have to come from out in the wings of the melting regime (see Figure 71 ), and so $P L 92$ point out that an important and probably prohibitive physical aspect to this model is that there would have to be a mechanism that focused these extra melts from the most distant parts of the melting regime into the central axis.

A second possible model for these aspects of local trace element variability, however, stems from another aspect of the occurrence of the steep trends. The best examples occur either in the vicinity of hot spots, or on the EPR. In both of these environments there is evidence for enricl.ed veins - though possibly on different scales. We saw from the melting models with $\mathrm{Na}_{2} \mathrm{O}$ that the increase in source abundance in such veins overwhelms any dilution effect from increased extents of melting, so that melting of such veins should lead to anomalously enriched compositions. Variable mixing of melts from the veins and from the surrounding more depleted mantle might lead to the steep trends in Figure $69 \mathrm{~b}$. This undoubtedly accounts for some of the data, particularly where there is a correlation between incompatible element abundances and the radiogenic isotope ratios (e.g., ${ }^{87} \mathrm{Sr} \beta{ }^{86} \mathrm{Sr}$ ) such as we observe for portions of the EPR.

Therefore local trace element variations for the most incompatible elements vary more than one would expect from the major element models. Two possible explanations are mixing of low degree melts from the wings of the melting regime, or melting of small-scale heterogeneities. Evidence from $\mathrm{Th}-\mathrm{Ra}-\mathrm{U}$, however, suggests there may be other processes exerting important effects of which we are not yet cognizant. Steep trends have not yet been discovered on normal sections of the Atlantic, but are common in the Pacific and where there are hot spot influences. Although these various major and trace element aspects of local variability are puzzling, they begin to make some sense when one considers the overall importance of spreading rate in influencing local variability.

\section{Significance of SpREAding Rate: \\ A KEY TO UNDERSTANDING THE LOCAL TRENDS?}

Many of the important aspects of ocean crust formation correlate or seem to be affected by spreading rate, such as the striking associations of rugged topography and rift valleys with slow-spreading ridges, and gentle topography and axial swells with fast-spreading ridges. Physical models predict spreading rate-dependencies on the pattern of melt and mantle flow [e.g., Scott and Stevenson, 1989; Spiegelman and McKenzie, 1987], which may influence the composition of melt formed and the volume of melt segregated to form the oceanic crust. It has been suggested that spreading rate correlates with crustal thickness for ridges far from hot spots [Reid and Jackson, 1981], with basalt major element composition [Bass, 1971; Morel and Hekinian, 1980], with isotopic heterogeneity [Cohen and O'Nions, 1982; Batiza, 1984], with gravity characteristics [Small and Sandwell, 1989], and with certain aspects of tectonic and magmatic segmentation [Schouten et al., 1985; Dick, 1989; Sinton et al., 1991]. We have found that the local vectors of compositional variability exhibit a striking contrast between slow- and fast-spreading ridges. Other aspects of MORB chemistry, however, such as the global correlations, appear to 

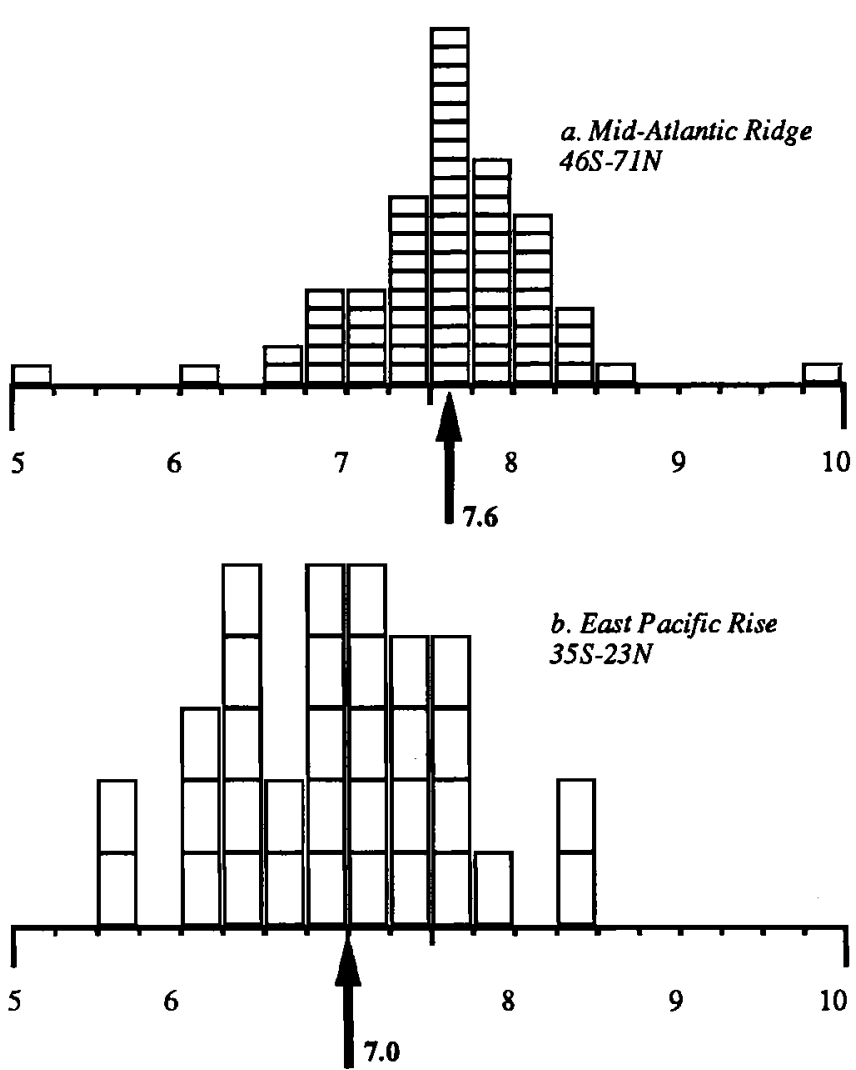

Fig. 72. a) Histograms of $\mathrm{MgO} w \mathrm{w} \%$ in samples from the Mid-Atlantic Ridge (46 ${ }^{\circ} \mathrm{S}-71^{\circ} \mathrm{N} ; 527$ analyses) and East Pacific Rise $\left(35^{\circ} \mathrm{S}-23^{\circ} \mathrm{N}\right.$; 400 analyses). b) Histograms of $\mathrm{MgO}$ wt\% averaged per degree latitude for the individual analyses shown in Panel (a). Data from the Smithsonian Institution Catalog of Basaltic Glasses [W. Melson, pers. comm.], Sinton et al. [1991] and references in Figures 21 and 24.

be independent of spreading rate. In the following, we examine which aspects of chemical variability correlate with spreading rate and which do not, and consider the implications. The implications include a possible model to account for the difference in chemical systematics between the MAR and EPR.

\subsection{Spreading Rate and Global Chemical Variability}

The first major observation is that the global correlations (Figure 15) are independent of spreading rate. Virtually the entire range of $\mathrm{Na}_{8.0}$, for example, occurs along the slowspreading northem Mid-Atlantic Ridge [see Figure $2 \mathrm{KL} 87$ ]. In addition, basalts with high $\mathrm{Na}_{8.0}$ are recovered from the the slow-spreading Mid-Cayman Rise and the Southwest Indian Ridge, as well as the medium-spreading Australian Antarctic Discordance. Furthermore, the superfast spreading southem EPR erupts basalts with a limited and intermediate range of $\mathrm{Na}_{8.0}$ values [Sinton et al., 1991]. Thus, there is no apparent correlation between $\mathrm{Na}_{8.0}$ and spreading rate.

There are important aspects of the global major element data set, however, that do show systematic relationships with spreading rate: average $\mathrm{MgO}$ contents of basalts and overall compositional diversity. Figure 72 shows histograms for the $\mathrm{MgO}$ contents of basalts from the EPR and MAR. The histograms are presented in two ways: as raw data, and as averages by degree of latitude so that densely sampled regions are not over-emphasized. In both treatments of the data, EPR basalts are significantly displaced toward lower $\mathrm{MgO}$ (lower temperature) than Atlantic basalts. The lower mean $\mathrm{MgO}$ contents of the EPR probably reflects the presence of crustal magma reservoirs. These reservoirs serve as the locations of mixing of diverse magma batches that are emplaced in the crust, and also as locations for cooling. Thus it makes sense that regions of faster spreading give rise to both more homogeneous and more differentiated basalt compositions.

The second important aspect is that the total range of variability increases substantially as spreading rate decreases. When the data are averaged region-by-region and examined as a function of spreading rate, there is an increase in the total range of mean $\mathrm{MgO}$ contents with decreasing spreading rate (Figure 73). Thus, slow-spreading ridges include basalts that are very primitive (e.g. FAMOUS, Kane to Hayes, Iceland), moderately differentiated (e.g. Reykjanes Ridge, Azores platform), or very differentiated (e.g. Mid-Cayman rise). On the other hand, all the data sets from fast-spreading EPR segments show similar mean $\mathrm{MgO}$ contents and similar standard deviations. The exception to this generalization is the occurrence of very low $\mathrm{MgO}$ (high $\mathrm{SiO}_{2}$ ) volcanics such as andesites and dacites at fast-spreading ridges. Such rocks have not yet been observed from slow-spreading ridges, but are quite common on the Galapagos Rise [Byerly et al., 1976; Christie and Sinton, 1981; Fornari et al., 1983], and also along the East Pacific Rise. Occurrences on these ridges are usually associated with propagating rifts or other small non-transform offsets. Sinton and Detrick [1992], however, have recently independently considered the spreading rate dependence of $\mathrm{MgO}$, and suggest that intermediate spreading rates may have

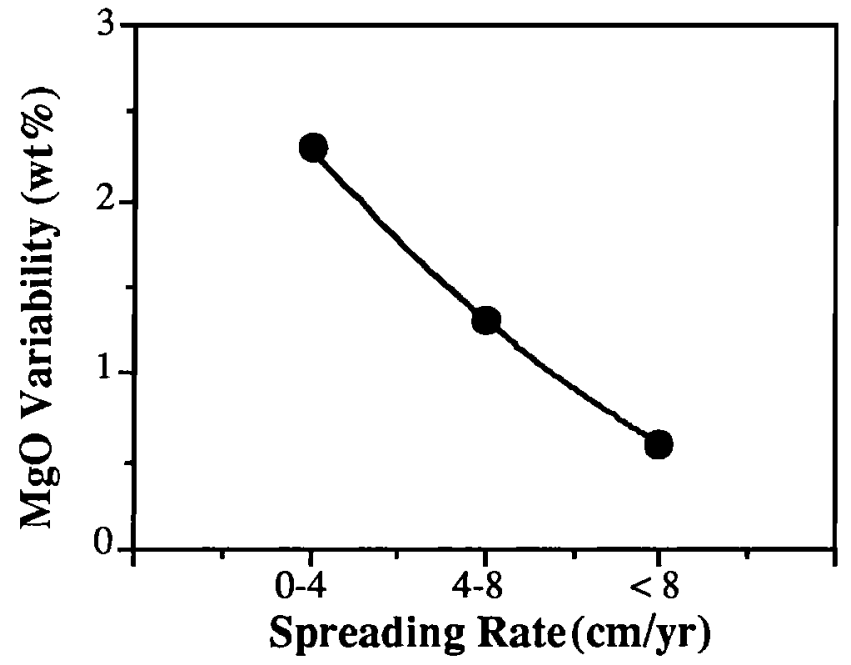

Fig. 73. Variability in $\mathrm{MgO}$ contents of basalts vs. spreading rate for the same data set as Figure 72 with the addition of data from intermediate spreading rate ridges. The variability is the total range in regional averages of $\mathrm{MgO}$ for more than $100 \mathrm{~km}$ segments within each of the three spreading rate intervals. For example, all lengths of the EPR have very similar mean $\mathrm{MgO}$ contents, while slow-spreading ridges range from very primitive to very evolved compositions. This variability does not include the high $\mathrm{SiO}_{2}$ samples that occur periodically on intermediate and fast-spreading ridges. 
the maximum variability, being in a transitional regime between the contrasting crustal fractionation systems of slow and fast-spreading ridges.

Many other chemical indices in addition to $\mathrm{MgO}$ exhibit an inverse relationship between diversity and spreading rate. For example, as noted above, slow-spreading ridges encompass the entire range in $\mathrm{Na}_{8.0}$ values (Iceland vs. Mid-Cayman rise), while fast-spreading ridges from the EPR show only intermediate values of $\mathrm{Na}_{8.0}$. Batiza [1984] suggested that variability in ${ }^{87} \mathrm{Sr} /{ }^{86} \mathrm{Sr}$ correlates inversely with spreading rate, (although a more recent study did not support this finding [White et al., 1987]).

This inverse relationship is not restricted to chemical composition. It is true, for example, of variations in depth: slow-spreading ridges include the entire depth range, while fast-spreading ridges are almost always between 2500 and 3000 meters. It is also true of ridge morphology. Fast-spreading ridges look alike, while slow-spreading ones can vary from deep rift valleys, such as near the Kane Fracture Zone, to very small rift valleys, such as near the Azores, to none at all on the Rekykjanes Ridge or Kolbeinsey Rise. Thus one of the primary observations of spreading rate dependence is that on a regional basis, slow spreading ridges are highly variable in chemistry, morphology and depth, while fast-spreading ridges show litle variation, with depth and regional chemistry at mean levels with respect to ridges as a whole.

\subsection{Spreading Rate and Local Variability}

As discussed at length above, there are striking differences between major and trace element variability on slow- and fastspreading ridges. The local trends of composition are completely distinguishable, and the expression of trace element heterogeneities is strikingly different as well. On well-studied slow-spreading ridges such as the northem MAR, the trace element signature is bimodal. Some regions are dominated by hot spot effects and erupt exclusively enriched basalts, while other long lengths of ridge can erupt exclusively depleted normal MORB compositions. On the EPR, in contrast, transitional and enriched MORB occur frequently, with no attribution to hot spot effects. The local trends in the Atlantic occur independent of the local mantle heterogeneity, while on the EPR the major element and mantle heterogeneity signals seem to be closely linked. These are such striking contrasts that they demand some sort of simple explanation.

A direction for an explanation comes from considering more carefully the spatial distribution of trace element patterns that occur along the MAR and EPR. We begin with consideration of two "normal segments" along each ridge, one between $8^{\circ}$ and $14^{\circ} \mathrm{N}$ on the EPR [Langmuir et al., 1986], and the other between the Kane and Hayes Fracture Zones on the MAR. Both these regions are well sampled, are not influenced by known hot spots, and are at average axial depths for ridges, with similar $\mathrm{Na}_{8.0}$ contents. The sole obvious difference between these two normal segments of ridge is that their spreading rates differ by a factor of three.

Figure 74 presents the contrasting histograms of $\mathrm{Ce} / \mathrm{Sm}$ ratios for about 50 samples that have been analyzed for REE from each region. The Kane-Hayes samples are all light REE depleted, with $\mathrm{Ce} / \mathrm{Sm}$ ratios of $2.71 \pm 0.27$. The EPR samples are much more variable, and have a much higher mean $\mathrm{Ce} / \mathrm{Sm}$ for the entire population (3.74 \pm 0.67$)$. These are striking contrasts for two reasons. First, both these ridge segments should be on the "baseline" for normal MORB removed from hot spots, since they have similar values of $\mathrm{Na}_{8.0}$. Second, as just pointed out, standard deviations for most parameters are much higher for slow-spreading than fast-spreading ridges, yet here, for almost a thousand $\mathrm{km}$ of ridge, the MAR data set is much more homogeneous. This contrast is apparent in Figure 75, which shows how $\mathrm{Ce} / \mathrm{Sm}$ varies with distance along each ridge. The higher and more variable values for the EPR are clear.

The extensive data set of Jean-Guy Schilling for much of the N. Atlantic can be used to consider whether these relationships are unusual [Schilling et al., 1983]. To make this comparison, it is necessary to convert Schilling's data for $\mathrm{La} / \mathrm{Sm}$ ratios to $\mathrm{Ce} / \mathrm{Sm}$ ratios, since he usually does not analyze $\mathrm{Ce}$, and the Kane-Hayes and EPR data sets frequently have no La. In Schilling's data set, $\mathrm{Ce} / \mathrm{Sm}$ and $\mathrm{La} / \mathrm{Sm}$ correlate very well $(\mathrm{R}=$ .988 ), so that a conversion factor can be applied to the $\mathrm{La} / \mathrm{Sm}$
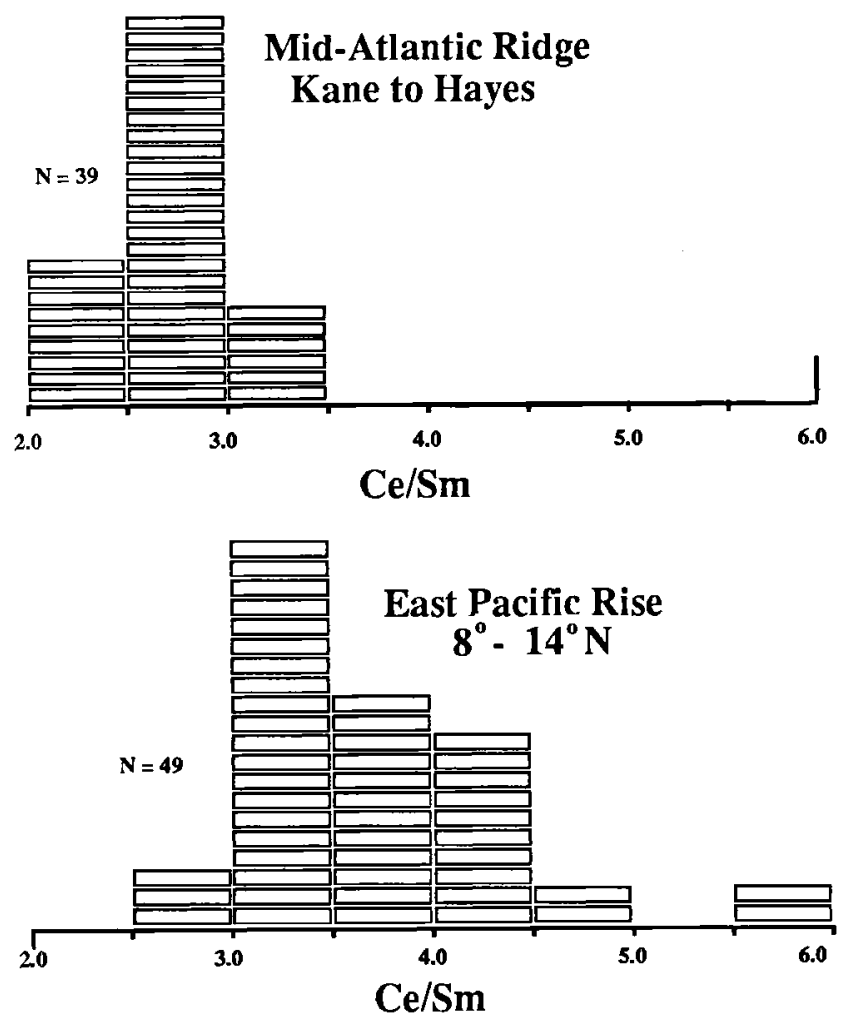

Fig. 74. Comparison of the distribution of REE data from normal sections of the Mid-Atlantic Ridge and the East Pacific Rise. The MAR data are from more than $1000 \mathrm{~km}$ of ridge between 24 and $34^{\circ} \mathrm{N}$, extending from the Kane to the Hayes Fracture Zones [Langmuir, unpublished data by plasma spectrometry]. The EPR data are from $\sim 700 \mathrm{~km}$ of ridge from $8^{\circ} \mathrm{N}-14^{\circ} \mathrm{N}$ [Bender and Langmuir, unpublished data by isotope dilution]. Over these distances, the MAR data for a normal section of ridge are both more depleted and more homogeneous than the EPR data. 


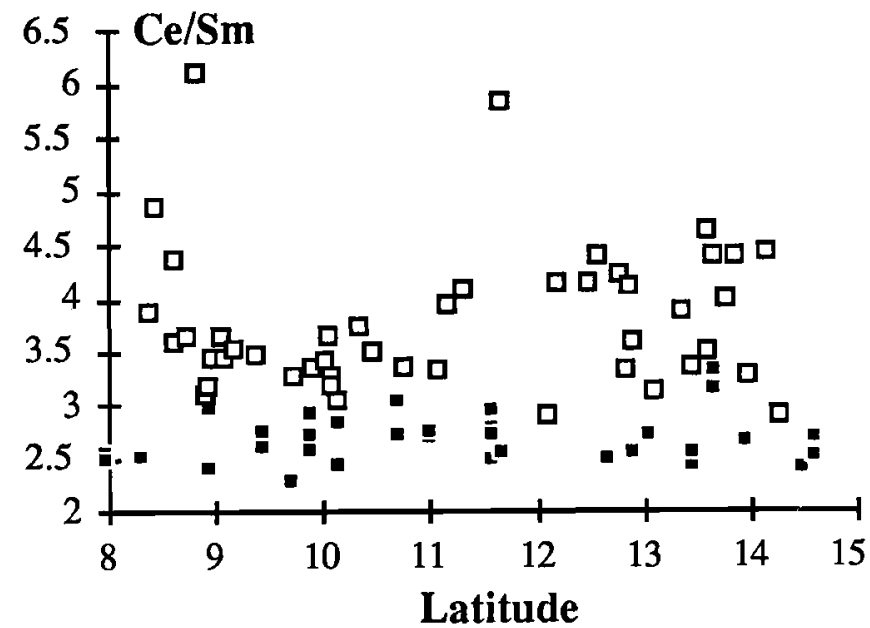

Fig. 75. Distribution of $\mathrm{Ce} / \mathrm{Sm}$ ratios with distance along the EPR and MAR, using the same data set as Figure 74. Latitude is for the EPR data set. MAR data are at the same scale, but latitudes are not shown. Open squares are for the CHEPR EPR data set, closed squares are from Kane to Hayes. Note that the high $\mathrm{Ce} / \mathrm{Sm}$ samples from the EPR are widely distributed along the ridge. The MAR does contain even more enriched samples than the EPR, but these are localized in specific hot spot regions (see Figure 68). No enriched samples were recovered on axis in the Kane to Hayes region.

data to arrive at $\mathrm{Ce} / \mathrm{Sm}$ values. The conversion is $\mathrm{Ce} / \mathrm{Sm}=$ $2.025 * \mathrm{La} / \mathrm{Sm}+0.9$.

Schilling's data set shows that for a much greater length of ridge than considered above, normal Atlantic segments are both more depleted and more homogeneous than the EPR. There are six normal segments of three degrees of latitude in Schilling's data set. Each of these segments has a mean $\mathrm{Ce} / \mathrm{Sm}$ less than 2.9, much lower than the EPR data, and does not contain enriched basalts - hence the standard deviations are low. Other comparable data sets from the EPR are not available, but the distribution of data from the southern EPR reported by Sinton et al. [1991] appear to have a similar appearance to the northern EPR data. The random distribution of relatively enriched rocks is a striking characteristic of the southern as well as northern EPR data sets. Therefore the contrasting characteristics observed in the $8-14^{\circ} \mathrm{N}$ and Kane to Hayes regions appear to be general phenomena associated with the EPR and MAR.

Of course, the MAR also contains extensive lengths of ridge near hot spots that consist of enriched MORB. The population is bimodal, with a clear separation between enriched and depleted lengths of ridge (Figure 68). Where enriched basalts occur, the enrichment often appears gradually, over long gradients, such as that south of the Azores, where $\mathrm{Ce} / \mathrm{Sm}$ increases from 2.5 to 9 progressively over seven degrees of latitude. Therefore the relative homogeneity of normal sections of the MAR contrasts with the high standard deviation for the MAR as a whole, which comes from the presence of both hot spot-influenced and "normal" sections of ocean ridge.

In view of these contrasts between the MAR and EPR, a most striking observation is that the MEAN Ce/Sm for the two ridges is identical, if both enriched and depleted portions of the MAR are included. This is apparent in Figure 76, which illustrates the dispersion of the entire N. Atlantic data set, and that mean MAR and EPR values are identical. The means are the same whether one takes the entire Atlantic data set from $22^{\circ} \mathrm{N}$ to $72^{\circ} \mathrm{N}$ as individual samples, or divides it into equal latitude intervals to remove all sampling bias. To summarize: for ridge lengths of several thousand kilometers, the means from both EPR and MAR are the same. The Atlantic is more dispersed, with more enriched samples than occur anywhere on the EPR. However, for normal sections of ridge, the EPR is more variable in incompartible elements and contains more enriched compositions. Therefore in trace elements as in major elements, there is a striking contrast between these two beststudied ocean ridges.

\subsection{A Model to Account for Contrasts between EPR and MAR Major and Trace Element Data}

The major and trace element contrasts between MAR and EPR might be explained by the interaction of a constant plume flux with variable spreading rate. Consider that the plume flux into both ocean basins may be comparable. For the Atlantic, the basin is bounded by continents on all sides, is slow spreading, and has recently opened. The Pacific, on the other hand, has a

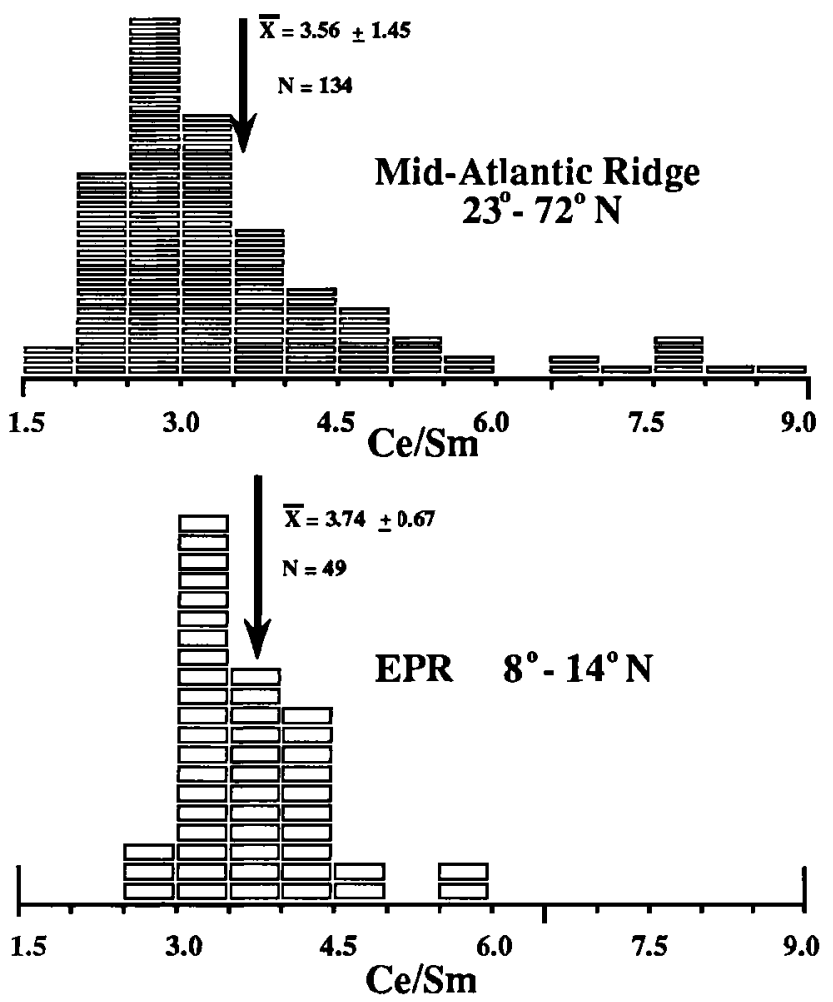

Fig. 76. Comparison of the EPR data set from Figure 74 with data from the more than $5000 \mathrm{~km}$ of the MAR, which includes several N. Atlantic hot spots. Data are from Kane to Hayes and also from Schilling et al. [1983]. The distribution of the individual samples for the two ridges is very different, but the mean is the same. Compared to the northern MAR as a whole, the EPR data are more homogeneous. Mixing of the $N$. Atlantic data could give rise to the EPR distribution. The same contrast is present in the data whether the data are examined as individual samples or by window of latitude, so the result does not depend on sampling bias. 
very long history of spreading, and is a large basin with very fast spreading rates. Processing and hence mixing of the mantle will be much more rapid and efficient in the Pacific than the Atlantic. These factors could lead to plumes becoming mixed on a fine scale into the Pacific mantle, and remaining clearly separated in the Atlantic mantle. Faster spreading would dilute and mix the plume flux more efficiently into the upper mantle prior to melting at the spreading center.

At the same time, the melting process is strongly affected by spreading rate. On slow-spreading ridges, there are large gradients in depth along strike, suggesting a complex and diverse melting regime that varies substantially over intermediate distances [e.g. Lin et al 1990]. Therefore along a spreading segment, melts are erupted from diverse portions of the melting regime. On fast-spreading ridges, the melting regime is two dimensional and does not vary nearly as much along strike. Melts undergo a similar history and mixing all along the ridge. Small scale heterogeneities can be preserved because of rapid melt processing and vertical transport along and possibly across the ridge as well.

These two concepts explain qualitatively the major observations from the MAR and EPR. The MAR would have some very depleted and homogeneous portions, which are not influenced by hot spots. Other portions of the MAR would have relatively pure hot spot signature, leading to large depth and chemical anomalies. Temperature anomalies, variations in depth, crustal thickness and extent of melting would all be preserved. The slow-spreading rate and frequent ridge offsets associated with it leads to substantial variations along strike, and much variability within the melting regime. This gives rise to the major element local trends as a result of variable melting processes, strongly influenced by the pronounced tectonic fabric of the ridge. Because of the relative isolation of mantle influenced by the hot spots, large scale heterogeneities would be incompletely mixed, so that large blocks of mantle could have their own heterogeneous composition, and their own local melting trend. This may be the case for the Oceanographer data. Of course, there is evidence for some small scale heterogeneity around hot spot regions in the Atlantic. This could result from the partial mixing that takes place as the plume material interacts with the rest of the mantle.

For the Pacific, the plume component has become distributed on a small scale through the mantle. As Allegre and Turcotte [1986] pointed out in a different context, mantle mixing often leads to thin layers of material that have been folded and refolded, giving rise to ubiquitous small scale heterogeneities. This material could then give rise to random enriched basalt. But this component is not present everywhere. In a region where it does occur, such as the Pacific, the basalt is never as enriched as the most enriched basalts from the Atlantic, because the plume component residing in the enriched layers is always diluted by the surrounding depleted mantle matrix.

At the same time as small scale heterogeneity contributes to compositional diversity of erupted basalts, melting processes alone beneath the EPR seem to have limited effect. The temperature of the mantle appears to be uniform over large distances along strike, possibly due to more efficient mantle processing or tectonic history. And ridge segmentation is subdued in comparison to slow-spreading ridges. Thus, long and short wavelength effects on the EPR are muted in comparison to the Atlantic, and there is no local trend caused by melting processes. Instead, the local trend is a consequence of small scale heterogeneity.

This model explains many aspects of EPR volcanism - the lack of a slow-spreading major element vector, the random distribution of enriched MORB, the lack of very enriched MORB. It also might explain the gradual gradients in chemistry observed in the southern EPR [Sinton et al., 1991]. Such long and smooth gradients might be the vestiges of incompletely mixed plume material. It could also explain why there is such a variety of compositions on many Pacific seamounts [e.g. Batiza and Vanko, 1984; Zindler et al., 1984]. If the sub-Pacific mantle is veined, seamounts would sample all this variety without any of the homogenizing influence of subaxial magma chambers.

There are also predictions for the MAR. For example, the lack of this veined material would suggest that off-axis seamounts along normal portions of the MAR removed from hot spots will not contain enriched MORB - in contrast to the common experience in the Pacific. One might also expect preservation of older heterogeneities in the Atlantic, since mixing is less efficient.

This model also appears to account for some of the differences in the details of the global correlations between ocean basins. Figure $16 b$ showed that Pacific ridges were offset to lower $\mathrm{Fe}_{8.0}$ for the same $\mathrm{Na}_{8.0}$ in comparison to the Kane to Hayes region in the Atlantic. The Atlantic as a whole, however, is characterized by normal MORB regions with relatively high $\mathrm{Fe}_{8.0}$, and hot spots with relatively low $\mathrm{Fe}_{8.0}$. The combination of these two would lead to intermediate levels of $\mathrm{Fe}_{8.0}$ - just what is observed for the Pacific data. Therefore the same model that accounts for the gross features of the data, explains some of the detailed complexities of the global correlations.

Although this model is consistent with many aspects of the major and trace element data, and the spatial occurrence of the data along the ridges, there are important additional issues. First, the differences between the EPR and MAR may have multiple causes. Some may result from spreading rate, but others may be due to the differing history and tectonic environment of the two ocean basins. For example, the ubiquitous small scale heterogeneity of the EPR is also apparent on the slower-spreading Juan de Fuca, Endeavour and Explorer Ridges in the northern Pacific [e. g. Michael et al., 1989; Karsten et al., 1990], so it appears this characteristic may apply to other parts of the Pacific basin, though apparently not the Galapagos Rise [Schilling et al., 1981; Sinton et al, 1991]. Second, hot spots themselves are variable, and have different major element and incompatible element signatures. $\mathrm{Ce} / \mathrm{Sm}$ is a neutral trace element parameter to use for it is a ratio that is enriched in all hot spots. Trace elements that are more incompatible are likely to show more complex relationships between the ocean basins. An 
encouraging aspect of the differences between the MAR and EPR is that they may provide a fingerprint with which spreading rates and histories of ancient crust that no longer makes up the sea floor can be inferred.

\section{CONCLUSIONS}

One of the aims of this paper was to develop further the systematics and framework of the chemical variations of MORB on various scales, for the clear statement of the systematics provides the relationships that quantitative models need to explain. Correction of the global data set for interlaboratory biases reveals a significant amount of structure in the global correlations of $K L 87$. Back-arc basins and many hot spots separate cleanly from the normal MORB field, and the normal MORB form a coherent array that is useful for constraining melting models. There are significant if subtle differences among the different ocean basins, and striking differences in the distribution of the data as a function of spreading rate. On a smaller, local scale, the contrasts between the local trends in major and trace elements for the EPR and slow-spreading ridges are very clear. The relationships among these local trends, spatial position along ridge segments, and temporal variability, however, are still not well known. The relationship between major and trace elements for the slow-spreading local trends also needs much better definition. These areas of investigation are likely to lead to important new developments over the next decade.

A second aim of the paper was the development of quantitative models for mantle melting and fractionation. Although much remains to be done in this direction, the models provide a useful framework in which to carry out detailed evaluation of global and local data and the processes that give rise to them.

Interaction between the observations and the models leads to the following conclusions concerning melt formation and segregation beneath ocean ridges, and the generation of the diversity of ocean ridge basalts.

\section{Mantle temperature variations are important.}

The global vector of variability can be explained quantitatively by isentropic melting of a homogeneous mantle that intersects the mantle solidus at different pressures as a result of temperature variations. Mantle temperature differences exert a major control on MORB chemistry, and must be considered as one of the important variables of melting and melt segregation. Major element heterogeneity is ruled out as a cause of the global correlations for major elements.

II. Continuous melting with low residual porosity is indicated by both the basalt and abyssal peridotite data.

The high $\mathrm{FeO}$ contents of the global correlations and the large $\mathrm{FeO}$ differences among regions preclude equilibrium melting. Some of the most depleted peridotite compositions also are difficult to reconcile with purely equilibrium melting [Johnson et al., 1990]. Existing data, however, cannot place firm constraints on values of the residual porosity, because of the uncertainties in the calculations and the details of the melting process.

III. Melts can be extracted from a range of pressures within the mantle without substantial low pressure re-equilibration prior to emplacement in the crust. They must be extracted rapidly and efficiently, and yet mix prior to eruption.

The high mean pressures of formation indicated in MORB major element compositions require the extraction of melts from deep in the melting regime without substantial modification. An even stronger constraint is given by the positive correlation between pressure of melting and extent of melting that is required to model the global vector. This relationship can come about only if melts are delivered from great depth to the surface without substantial re-equilibration during ascent, and suggests that melts form and pool over a range of pressures extending from near the solidus to near the top of the melting regime.

Conclusions II and III provide rigorous constraints for porous flow models with low porosity, because slow travel along small channels may inevitably lead to reaction upon ascent and re-equilibration at low pressures. Transport must be efficient, with minimal reaction during ascent. This suggests channelized flow and rapid ascent.

IV. Petrology does not highly constrain the physical shape of the melting regime because many different shapes lead to identical petrological results.

Active upwelling vs. passive upwelling, however, should have different petrological consequences. Detailed petrological studies of individual ridge segments coupled with seismic studies of crustal thickness should provide quantitative tests of melting models as they apply to ridge segments and to ridge segmentation.

\section{The contrasting local vectors on slow-spreading ridges and} the EPR are very regular in their occurrence, and result from mantle processes.

The melting column model of $K L 89$ does not quantitatively account for the local trends observed on slow-spreading ridges or the EPR. Fractionation models, including high pressure fractionation, may contribute to the local vector in certain regions, but also fail to quantitatively account for the local vector. All fractionation models are inconsistent with the large variations in $\mathrm{Sr}$ that usually occur along with the major element variations. Therefore the local trends have to do with melting processes in the mantle. Alkali basalts from ocean islands also exhibit a local trend similar to that observed on slow-spreading ridges. The melting models fail completely to produce alkali basalts by small extents of melting at high pressure. Therefore we know there are aspects of the melting problem that are not adequately treated with current models. Certainly one of these aspects is volatile content. $\mathrm{CO}_{2}$ may play an important role in melting beneath ocean ridges.

If the local vector is produced by different extents of melting within the melting regime, then it may become a tool to "map" the melting regime, showing from where in the mantle melts are delivered to the crust, and the spatial relationship between position on the ridge axis and position within the 
melting regime. In addition, the magnituce of the local vector may constrain the extent of mixing of melts in the melting regime or in the crust.

The local variability of chemical data from the Pacific is as systematic as that from the slow-spreading ridges, but define a different array in compositional space. We propose that this array is produced by melting of a mantle that is heterogeneous on a small scale. Such heterogeneity is not observed on normal tidges in the Atlantic.

VI. There is no systematic global correlation between mean extent of melting and spreading rate. Spreading rate controls the standard deviation of variability of many parameters. however.

In the limiting case of extremely slow spreading, however, basalts may be derived by the lowest extents of melting. The clearest correlation with spreading rate is with the standard deviation of chemical variability, both for melting and fractionation processes. Faster spreading ridges appear to be associated with limited mantle temrerature variations, possibly due to more efficient mantle processing or tectonic history.

VII. Hot spots and small scale heterogeneities play an important role in controlling the chemical observations.

Heterogeneity in the Atlantic is on a 1 rge scale and relates to established hot spots. Heterogeneity on the EPR is mostly dispersed and ubiquitous. The mean $\mathrm{Ce} / \mathrm{Sm}$ ratios of basalts from the two ridges are identical. The contrasts between the MAR and EPR may result from the sub-Pacific mantle having mixed in hot spot material more efficieritly. This idea also helps to explain the different local trends of the MAR and EPR. The EPR is of constant depth, and or axis exhibits few variations that relate directly to the meling regime. Small scale heterogeneities control most of he major and trace element characteristics of the Pacific lucal vector. On the MAR, hot spot material is separaled, and creates long wavelength gradients. The complexity and diversity of melting environments of a slow-spreading ridge causes the major element characteristics of the slow-spreadiig local vector.

Small scale veins may also play an important role in controlling the way the mantle melts, the distribution of melt within the melting regime, the relationship between pressure and extent of melting, the efficiency of extraction, and melt focusing. Extraction from a heterogeneous medium could have important physical and chemical effects that influence basalt compositions and the creation of the crust in general.

VIII. There is an unknown process that influences highly incompatible elements.

The fractionation of $T h$ from $U$ coupled with the $T h$ isotopic disequilibrium requires fractionation of these highly incompatible elements on a time scale of less than 100,000 years. The even larger $\mathrm{Ra}$ fractionations, coupled with new experimental data suggesting $U$ is more incompatible than $T h$, show that equilibrium (or fractional) melting cannot be the cause of these fractionations. Therefore there must be some additional diffusive or disequilibrium process that is influencing the distribution of these elements in the melting regime. Surprises for melting models may be in store.
Finally, we note that the various chemical constraints seem to require a combination of characteristics that are not fit by a single model of the melting regime. Meliing that is close to fractional suggests movement of small ceiree melts at low porosity. One would think these would interact with their surroundings. On the other hand, the mujor element evidence shows that some melts reach the surface without interacting with the mantle during ascent. The global systematics require extensive melt pooling. The local variability suggests melts can often ascend in small batches without being thoroughly homogenized.

Aspects of these diverse observations might be reconciled with a model in which the style of segregation changes during ascent. Porous flow with low porosity may apply deep in the system. Progressively larger conduits may channel the melt at shallower levels. Ultimately, large channels may allow rapid movement of the melt to the surface without further interaction with the surrounding mantle. This hierarchy of melt delivery systems may require different sorts of physical mechanisms for different levels of the system. It is also possible that the degree of communication and separation of the melt delivery systems along strike may vary with depth in the mantle and segregation style, which may themselves be influenced by the thickness, distribution and compositior of heterogeneous veins. A hierarchy of mantle flow and nelt delivery systems may aid in the explanation of the hierarchical segmentation that may exist along spreading ridges [Langmuir et al., 1986; Macdonald et al., 1988].

\section{APPENDIX A: Calculation of FeO, $\mathrm{MgO}, \mathrm{Na}_{2} \mathrm{O}$ AND TTO $_{2}$ DURING AdIABatic Melting OF THE MaNTLE}

The contents of $\mathrm{FeO}$ and $\mathrm{MgO}$ in mantle melts are constrained by the fact that olivine is always a residual phase. Hanson and Langmuir [1978] and Langmuir and Hanson [1980] used this fact to develop equations to calculate the possible concentrations of $\mathrm{FeO}$ and $\mathrm{MgO}$ that could be produced during mantle melting. Actual melting paths could not be calculated, but the field of possible compositions still provided useful constraints. The knowledge that olivine must be residual is quite powerful, for it means that the $\mathrm{FeO}$ and $\mathrm{MgO}$ contents of mantle melts will be constrained by the partition coefficients for these two elements between olivine and liquid. If these partition coefficients can be expressed as a function of temperature, pressure and composition, and the pressure, temperature and relevant other compositional variables are known, then the $\mathrm{FeO}$ and $\mathrm{MgO}$ content of the melt is fully constrained.

Two additional developments allow this method of calculation to be developed further: equations for $\mathrm{K}_{d}$ 's for $\mathrm{FeO}$ and $\mathrm{MgO}$ as a function of $\mathrm{P}, \mathrm{T}$ and liquid composition (see Appendix B), and the existence of a meiting function, which provides $T, P$ and the extent of melting for any pressure of intersection of the mantle solidus. These developments now allow a rigorous calculation of $\mathrm{FeO}$ and $\mathrm{MgO}$ contents in mantle melts for different pressures of intersection of the mantle solidus. Different melting functions and melting processes can be easily incorporated into the model. 
The calculation works as follows. For any mantle potential temperature, the melting function provides $T, P$ and $F$ as the mantle ascends, as detailed in section 4.2 We wish to determine six parameters: the $\mathrm{FeO}$ and $\mathrm{MgO}$ contents of the residue, olivine and liquid. We have as given the bulk mantle composition, $\mathrm{MgO}_{0}$, and $\mathrm{FeO}_{0}$. We have two equations for the $\mathrm{K}_{\mathrm{d}}$ 's for $\mathrm{MgO}$ and $\mathrm{FeO}$ between olivine and liquid as a function of pressure, temperature and liquid composition.

$$
\begin{aligned}
\mathrm{K}_{\mathrm{dMBO}_{\mathrm{O}}} & =\mathrm{MgO}_{\mathrm{O}} / \mathrm{MgO}_{\mathrm{L}}=\mathrm{f}(\mathrm{T}, \mathrm{P}, \mathrm{x}) \\
\mathrm{K}_{\mathrm{dFeO}} & =\mathrm{FeO}_{\mathrm{OL}} / \mathrm{FeO}_{\mathrm{L}}=\mathrm{g}(\mathrm{T}, \mathrm{P}, \mathrm{x})
\end{aligned}
$$

There is also one mass balance equation for each element:

$$
\begin{aligned}
\mathrm{MgO}_{\mathrm{o}} & =\mathrm{F}^{*} \mathrm{MgO}_{\mathrm{L}}+(1-\mathrm{F})^{*} \mathrm{MgO}_{\mathrm{s}} \\
\mathrm{FeO}_{\mathrm{o}} & =\mathrm{F}^{*} \mathrm{FeO}_{\mathrm{L}}+(1-\mathrm{F})^{*} \mathrm{FeO}_{\mathrm{s}}
\end{aligned}
$$

and the equation required by olivine stoichiometry:

$$
\mathrm{MgO}_{\mathrm{OL}}+\mathrm{FeO}_{\mathrm{OL}}=66.67 \mathrm{~mol} \%
$$

We need finally a relationship between the $\mathrm{Fe} / \mathrm{Mg}$ ratio of the olivine and the residue composition. In most cases, the $\mathrm{Fe} / \mathrm{Mg}$ ratios of the dominant mantle phases (olivine and two pyroxenes) are similar, and we take the $\mathrm{Mg} / \mathrm{Fe}$ ratio of the olivine and the total residual solid to be the same.

$$
\mathrm{MgO}_{\mathrm{OL}} / \mathrm{FeO}_{\mathrm{OL}}=\mathrm{MgO}_{\mathrm{s}} / \mathrm{FeO}_{\mathrm{s}}
$$

We therefore have six equations that relate the six unknowns. Solution of this set of equations leads to a quadratic with one element in the liquid as the unknown variable:

$$
\begin{aligned}
& 0=\mathrm{A}^{*} \mathrm{FeO}_{\mathrm{L}}{ }^{2}+\mathrm{B}^{*} \mathrm{FeO}_{\mathrm{L}}+\mathrm{C} \\
& A=F K_{d F o O}\left(1-K_{d F o} / K_{d M g O}\right) \\
& \mathrm{B}=\mathrm{K}_{\mathrm{dPeO}}\left(66.67 \mathrm{~F} / \mathrm{K}_{\mathrm{dM}_{\mathrm{g}} \mathrm{O}}-\mathrm{MgO}_{\mathrm{o}}-\mathrm{FeO}_{\mathrm{o}}\right)-66.67 \mathrm{~F} \\
& \mathrm{C}=66.67 \mathrm{FeO}
\end{aligned}
$$

All other solid and liquid compositions can then be determined from equations A1 - A6. These equations thus permit the straightforward calculation of liquid compositions for a variety of melting functions, melting mechanisms (e.g. fractional or equilibrium melting), source compositions and mantle temperatures. The one additional key piece of information that is required to carry out the calculation is the dependence of the partition coefficients on temperature, pressure and composition (these equations are developed in Appendix B).

It is important to recognize that these equations are not calculating liquid compositions when olivine is the ONLY residual phase, they solve the general problem as long as olivine is present as ONE of the residual phases. Equilibrium relationships between olivine and liquid apply independently of whatever other phases may or may not be present. A source of uncertainty in the calculation, however, is in equation (A6). This relationship applies well for plagioclase or spinel peridotite, where essentially all of the $\mathrm{FeO}$ and $\mathrm{MgO}$ is in olivine and pyroxenes, which have similar $\mathrm{MgO} / \mathrm{FeO}$ ratios. Garnet, however, is much more $\mathrm{FeO}$ rich, and a garnet-rich residuum would lead to a different equation in place of A6.
Since garnet melts out of the residue rapidly, this is not a serious problem for the conclusions of the present paper, but it may be important for small degrees of melting at higher pressures where garnet is an important residual phase. The effect of residual garnet will be to make the olivine more magnesian (less $\mathrm{FeO}$ ) than the total residue. This will cause $\mathrm{FeO}$ contents of the liquid to be slightly lower, as one would expect when an Fe-rich phase such as gamet is left in the residuum.

It is possible with present data to calculate three other elements, $\mathrm{Na}, \mathrm{Ti}$, and $\mathrm{K}$, using a $\mathrm{K}_{d}$ approach. $\mathrm{K}$ has very low partition coefficients in all mantle phases, and hence can be calculated as an incompatible element with very low D's. Clinopyroxene is the major host for $\mathrm{Na}$ and $\mathrm{Ti}$ in the mantle, so we have examined the experimental data set for clinopyroxene/liquid partitioning. These elements can also be treated as trace elements, and all that is required are equations for the dependence of the $K_{d}$ 's on temperature and pressure. Compositional effects are also likely to be important, but because these two elements are moderately incompatible, their D's are low in the mantle/melt system, and small errors in the $\mathrm{K}_{\mathrm{d}}$ s do not affect the liquid composition substantially after the extent of melting is greater than a few percent. The behavior of the partition coefficients is very important, however, for calculating residual mineral compositions. Whereas a variation in the $\mathrm{D}$ for $\mathrm{TiO}_{2}$ of 0.003 to 0.01 has little effect on the calculated melt composition at $10 \%$ melting, it causes variation of more than a factor of three in the residual solid composition. Because the emerging data on compositional variations in residual ultramafics may offer important constraints on melting processes [Johnson et al., 1990], it is important that the partition coefficients be determined accurately. Equations for these two elements have been determined based on over 100 experimental data from the literature (see references in the caption to Figure A1). Dependence on both temperature and pressure are important. The experimental data are presented in Figure Al, along with curves that show the equations used to calculate the appropriate $K_{d}$ 's as a function of $T$ and $P$. The equations are:

$$
\begin{aligned}
& \log _{10} K_{d}(\mathrm{Ti}, \mathrm{Cpx} / \mathrm{L})=(10000+90 \mathrm{P}(\mathrm{kb})) / \mathrm{T}^{\circ} \mathrm{K}-7.5 \\
& \log _{10} \mathrm{~K}_{\mathrm{d}}(\mathrm{Na}, \mathrm{Cpx} / \mathrm{L})=\left(5100+73 \mathrm{P}(\mathrm{kb}) / \mathrm{T}^{\circ} \mathrm{K}-4.48\right.
\end{aligned}
$$

In the calculation of liquid compositions, the contribution of orthopyroxene to the bulk partition coefficient is taken as a constant $(.008$ for $\mathrm{Na}$ and .01 for $\mathrm{Ti})$, and the modal clinopyroxene is decreased as melting proceeds assuming that it makes up $60 \%$ of the melt fraction.

The advantage of this $K_{d}$ approach to calculating mantle melt compositions is that many of the problems associated with performing mantle melting experiments, such as analyzing small melt pockets and equilibrating multiple phases, can be circumvented. For example, $\mathrm{K}_{\mathrm{d}}$ 's can be determined from experimental run products that consist of large quantities of melt and a small number of phases. This makes a wider variety of experiments available to characterize the $K_{d}$ s. In Appendix $B$, several hundred olivine/liquid pairs are considered, which 


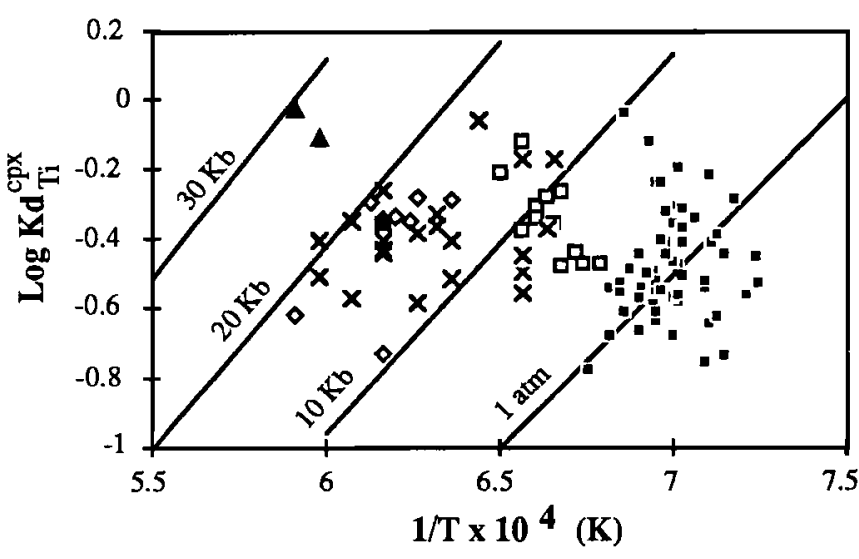

Fig. A1. Clinopyoxene/melt $\mathrm{K}_{\mathrm{d}}$ for $\mathrm{Ti}$ vs. inverse temperature for experiments on mafic systems. Data sources and symbols as in Figure 39. Although the temperature dependence is not well constrained, the absolute errors involved are small, because the range of temperature of the calculations is similar to that of the experimental data points, and the $\mathrm{K}_{d}$ 's vary over a small range. Since $\mathrm{Ti}$ is moderately incompatible in the mantle system, errors in liquid composition for moderate extents of melting are not strongly dependent on errors in the $K_{d}$ 's.

constitutes an order of magnitude more data than is available for other approaches to mantle melting calculations. Because these calculations are rigorous for any particular set of $K_{d}$ 's and melting function, they provide a useful test of other melting models that are based on empirical or qualitative reproduction of the relatively small experimental data set on multiply saturated liquids.

\section{APPEndix B: Olivine Liquid Partitioning as a FUNCTION OF TEMPERATURE, PRESSURE AND LIQUID COMPOSITION}

The dependence of the partition coefficients for $\mathrm{FeO}$ and $\mathrm{MgO}$ in olivine on pressure, temperature and composition is of major importance for the calculations presented in Appendix A. The behavior of the olivine $K_{d}$ 's for MORB compositions is fairly well known, at least up to $15 \mathrm{kbar}$ [e.g., Bender et al., 1978]. However, the mantle beneath ridges may begin to melt at higher pressures. In addition, these initial melts will have high alkali contents, which can have a significant effect on olivine partition coefficients [Roeder, 1974].

Ford et al. [1983] developed equations for the pressure-, temperature- and compositional-dependencies of olivine $K_{d}$ 's. Beatty [1992] recently investigated the temperatures of liquids in equilibrium with olivine, and developed equations for $K_{d}$ 's that are independent of temperature. These latter equations are strongly constrained by olivine stoichiometry [Langmuir and Hanson, 1981], and are not particularly useful for calculations of liquid compositions. However, the wealth of recent experimental data on a range of melt compositions can be used to refine the $\mathrm{K}_{\mathrm{d}}$ expressions developed by Ford et al. [1983].

In order to develop expressions for the olivine partition coefficients that are calibrated over the range of pressures and compositions likely for mantle melting, we have investigated over 250 experiments from the literature. These experiments span a range of pressures of up to $60 \mathrm{kbar}$, and a range of compositions including MORBs [Grove and Bryan, 1983; Grove et al., 1990; Bender et al., 1978; Walker et al., 1979; Tormey et al., 1987], highly alkaline basalts [Sack et al., 1987], lunar basalts [Grover et al., 1980] andesites [Grove et al., 1982], komatiites [Agee and Walker, 1990] and the ironand alkali-free CMAS system [Presnall et al., 1979]. Thus we have included an even wider range of compositions and pressures than are expected to occur in the melting regime.

Roeder and Emslie [1970] originally showed a linear relationship between $\log \mathrm{K}_{\mathrm{d}}$ and inverse temperature, which is what would be predicted from the thermodynamics of ideal solutions. A rough correlation between $\log K_{d}$ and inverse temperature is present in the large experimental data set, but use of this simple linear relationship between the $\log \left(K_{d}\right)$ and $1 / \mathrm{T}$ leads to substantial errors in some regions of compositional space (Figure B1). The average error for a fit based only on the temperature dependence for the $1 \mathrm{~atm}$ data is $15 \%$.

To investigate the compositional effects, we examined the data in small temperature and pressure windows to see if any obvious relationships were apparent. The spread in the data was clearly related to pressure and to variations in the alkali, $\mathrm{Si}$ and $\mathrm{Al}$ contents of the liquids. This relationship is apparent if one fits the $1 \mathrm{~atm}$ data with a linear $1 / T$ dependence, and then examines relationships between the residuals from the $1 / \mathrm{T}$ fit and the liquid compositions. The residuals vary linearly with a compositional term, which includes a sum of the $\mathrm{SiO}_{2}, \mathrm{Al}_{2} \mathrm{O}_{3}$, $\mathrm{Na}_{2} \mathrm{O}$ and $\mathrm{K}_{2} \mathrm{O}$ contents of the liquid composition (Figure $\mathrm{B} 2$ ). The constants in the term were determined by fitting the residuals to the compositional variables. No other compositional terms improve the fit to the residuals.

We then took the fit to the 1 atm data including the compositional dependencies and determined the residuals of the high pressure data from the 1 atm regression. A simple correlation exists between the residual from the 1 atm fit and the pressure of the experiment for geological compositions (Figure $\mathrm{B} 3 a$ ). Thus, in addition to the temperature and compositional dependencies, a pressure dependent term must be included. Some of the experiments were in simple systems,

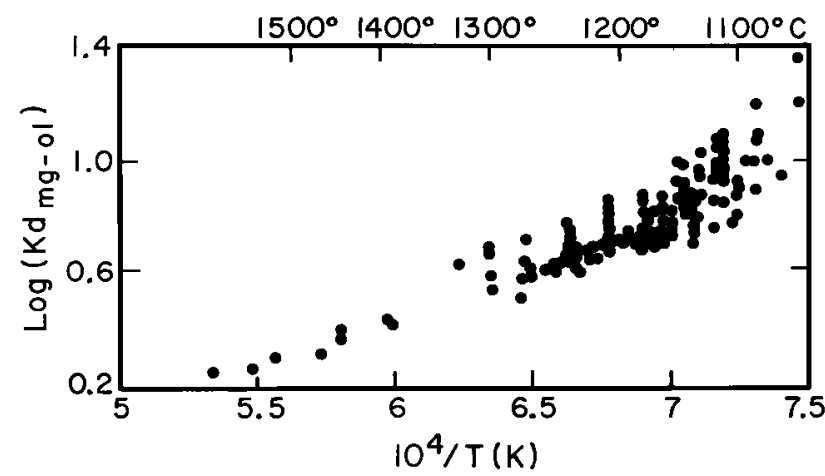

Fig. B1. The entire experimental data set for olivine/melt $\mathrm{K}_{\mathrm{d}} \mathrm{MgO}$ plotted against inverse temperature. Although there is a rough correlation, the scatter is large. 


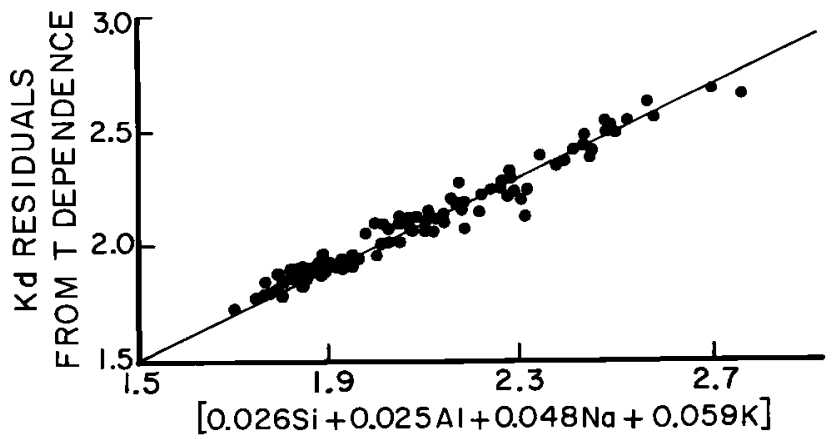

Fig. B2. Residuals of a linear fit between the $\mathrm{K}_{\mathrm{d}}$ 's for $\mathrm{MgO}$ and inverse temperature plotted against a linear combination of four elements in the liquid, for the $1 \mathrm{~atm}$ data alone. Compositional data are in cation mole percent.

however, with a much larger range of composition than occurs in the earth. From these experiments, it was clear that there was also a compositional term to the pressure dependence, due primarily to the amount of $\mathrm{Al}_{2} \mathrm{O}_{3}$, which probably changes its coordination in the liquid as pressure increases (Figure B3b). Thermodynamic considerations suggest that the $\log \left(\mathrm{K}_{\mathrm{d}}\right)$ should depend on $\mathrm{P} / \mathrm{T}$ rather than pressure alone. These aspects of the data suggest a multiple linear regression of the data with $\log \mathrm{K}_{\mathrm{d}}$ as the dependent variable and $1 / \mathrm{T}, \mathrm{P} / \mathrm{T}$, and $\mathrm{Na}_{2} \mathrm{O}, \mathrm{K}_{2} \mathrm{O}, \mathrm{SiO}_{2}$ and $\mathrm{Al}_{2} \mathrm{O}_{3}$ as the independent variables. Regression of the high

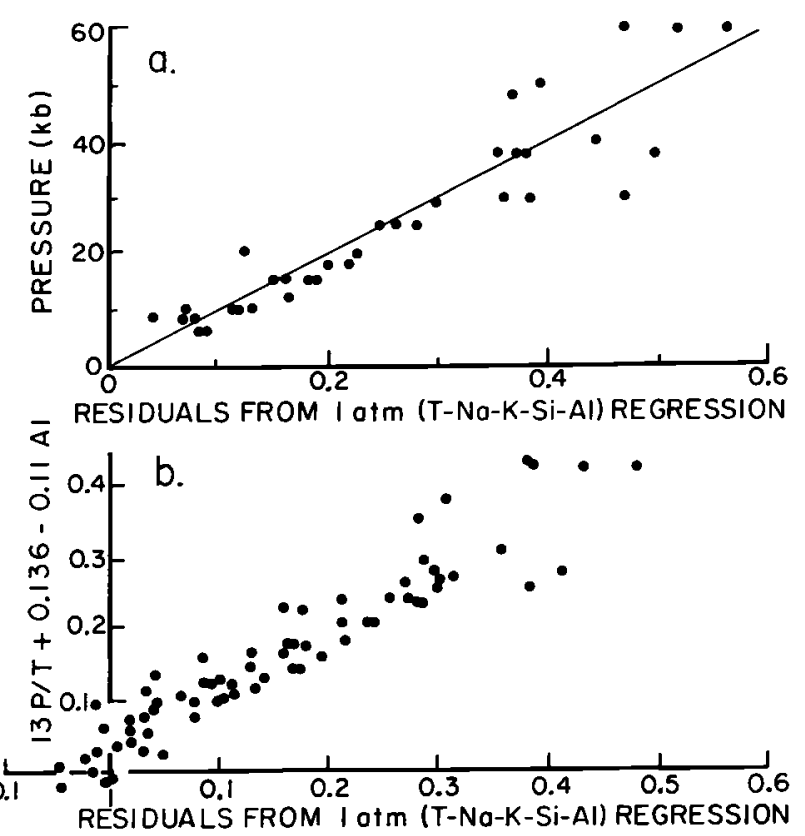

Fig. B3. a) The $1 \mathrm{~atm}$ expression for temperature and compositional dependence was applied to the high pressure data. Residuals from the 1 atm expression correlate linearly with the pressure of the experiment, except for those experiments in simple systems where there are large variations in $\mathrm{Al}$ content. b) Taking into account the $\mathrm{Al}$ effect and making the pressure term $\mathrm{P} / \mathrm{T}$, which is consistent with thermodynamic considerations, leads to a linear relationship with the residuals for the entire high pressure data set. pressure data shows that the Al dependence decreases as pressure increases. All terms in the equation are significant, and there are no additional compositional terms that improve the fit. The final equation is:

$$
\begin{gathered}
\operatorname{lnK} \mathrm{d}_{\mathrm{d}} \mathrm{Mg}= \\
4400 / \mathrm{T}+13 \mathrm{P} / \mathrm{T}+[.026 \mathrm{Si}+.025 \mathrm{Al}+.048 \mathrm{Na}+.059 \mathrm{~K}]- \\
.011 \mathrm{Al}(\mathrm{P}-.001) / \mathrm{P}+.136(\mathrm{P}-.001) / \mathrm{P}-3.231
\end{gathered}
$$

where the compositional terms are in cation mole percent and pressure is in kilobars. Average error of recovery of the $K_{d}$ 's is $4 \%$ for the entire data set. The error is evenly distributed among compositional groups; errors for the high pressure data alone are $4.2 \%$, for komatiites $4.5 \%$, and for high- $\mathrm{SiO}_{2}$ rocks, $5.5 \%$. Data from certain specific studies have systematically higher errors.

Although this equation covers a very broad range of compositions, it is not ideal for our purposes because it includes terms for $\mathrm{Si}$ and $\mathrm{Al}$, and we are unable at this time to calculate reliably the $\mathrm{Si}$ and $\mathrm{Al}$ contents of mantle melts. Much of the $\mathrm{Si}$ and $\mathrm{Al}$ dependence in equation $\mathrm{B} 1$ results from the data from simple systems such as CMAS, where the $\mathrm{Si}$ and $\mathrm{Al}$ variations are much larger than in natural data sets from real rocks. We carried out an additional regression of the data, therefore, leaving out the CMAS data. For this smaller yet geological applicable data set, $\mathrm{Na}$ and $\mathrm{K}$ alone account for most of the compositional dependence. Residuals from the $1 \mathrm{~atm} 1 / \mathrm{T}$ dependence correlate linearly with $\mathrm{Na}$ and $\mathrm{K}$ (Figure B4a). For
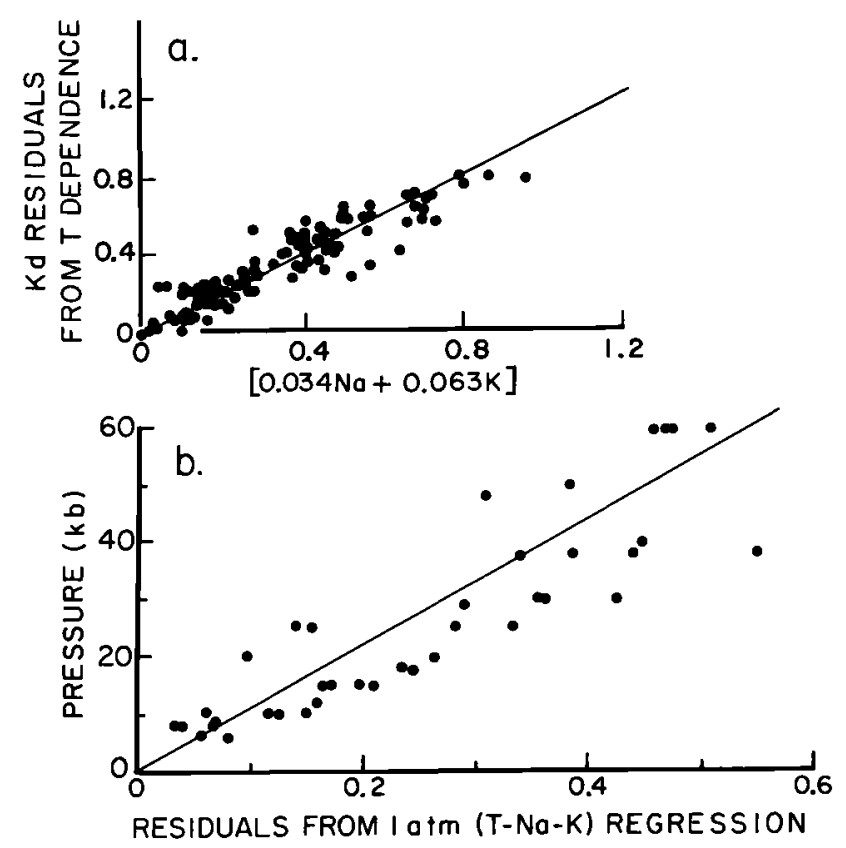

Fig. B4, a) Residuals of a linear fit between the $\mathrm{K}_{\mathrm{d}}$ 's for $\mathrm{MgO}$ and inverse temperature plotted against a linear combination of $\mathrm{Na}$ and $\mathrm{K}$ in the liquid (in cation mole percent). This relationship applies to geological compositions only, and not to the entire compositional range observed in simple systems. b) Linear relationship between residuals from the 1 atm temperature, $\mathrm{Na}$ and $\mathrm{K}$ fit and the pressure of the experiment. 
the high pressure data, residuals from the temperature and composition fit to the $1 \mathrm{~atm}$ data correlate linearly with pressure (Figure $\mathrm{B} 4 b$ ). The $\mathrm{K}_{\mathrm{d}}$ equation with only $\mathrm{Na}$ and $\mathrm{K}$ as the compositional variables is:

$\operatorname{LnK}_{d} \mathrm{Mg}=6921 / \mathrm{T}+.034 \mathrm{Na}+.063 \mathrm{~K}+.01154 \mathrm{P}-3.27$

This equation, which is used for the $\mathrm{Fe}$ and $\mathrm{Mg}$ modeling reported in the text, recovers the data with an average error of $5.3 \%$.

The magnitude of these errors needs to be considered in the context of interlaboratory bias, experimental errors, and the variability observed in the natural data that we are trying to explain. Consideration of analyses of similar samples by different microprobes indicates that interiaboratory biases can be significant. For example, the $\mathrm{MgO}$ contents determined by the Smithsonian microprobe [Melson et al., 1977] are systematically $6 \%$ lower than values determined at Lamont [Klein, 1989]. Thus errors of $5 \%$ in the $K_{d}$ 's may be as accurate as can be expected given that several different microprobes were used to analyze the experimental $\mathrm{n} n$ products, and that there are additional uncertainties in the reported temperatures and pressures of the experiments. The intal range in $\mathrm{Fe}_{8.0}$ that we are trying to understand is $50 \%$ of the mean $\mathrm{Fe}_{8.0}$ value, and thus is large relative to the errors for the $K_{d}$ expression. Furthermore, the relative variations calculat:d for the $\mathrm{FeO}$ and $\mathrm{MgO}$ contents of mantle melts will be mse precise than the absolute values, and it is the relative differences that largely constrain the models considered.

Finally, it should be noted that there is almost no experimental partitioning data on volatile-bearing systems. Since carbon and hydrogen are potentially important components in the mantle, particularl at low extents of melting, the effects of volatile species on partitioning at high pressures for the appropriate compositions is important future work.

\section{APPENDIX C: MODELING COMPLEX DIFFERENTIATION PROCESSES}

Much of the compositional variability observed in MORB can be attributed simply to low-pressure crystallization processes (see section 3.1). In addition, some aspects of the residual variability may result from more complex fractionation processes. These may include (a) magma evolution in periodically replenished magma chambers [O'Hara, 1977], where a magma chamber can be modeled simply as a holding tank with inputs and outputs; (b) in situ fractionation [Langmuir, 1989], where solidification takes place along the margins of a magma chamber in a temperature gradient that extends from solidus to liquidus; and (c) highpressure fractionation, which has been suggested to be an important factor in MORB evolution [e.g., Bender et al., 1977; Elthon et al., 1982]. To evaluate the importance of these fractionation processes in contributing to the diversity of MORB compositions, it is necessary to quantitatively examine their chemical effects.

\section{C.I Periodically Replenished Magma Chambers (PRMC's)}

There is abundant petrographic and geochemical evidence for magma mixing in MORB [e.g., Kuo and Kirkpatrick, 1982; Dungan and Rhodes, 1978] and common sense argues for replenishment of magma chambers when they exist. In the model of periodically replenished magma chambers [ $O^{\prime} H a r a$, 1977; O'Hara and Matthews, 1981], magmas undergo constant recharge, mixing, crystal fractionation and eruption, and melts evolve to steady state compositions if input and output parameters remain constant. The steady state is a cycle through two end-member compositions - one that occurs after replenishment with a new pulse of magma and mixing with the magma already in the chamber, and the other that occurs after crystallization in the chamber, just before the addition of the new magma pulse. O'Hara's original treatment dealt with compositions after crystallization and before mixing, but compositions after mixing and before crystallization may also be important.

The net chemical effects of periodically replenished magma chambers can be related simply to the liquid lines of descent for equilibrium and fractional crystallization in a closed system [Langmuir, 1980; 1989]. Steady-state liquid compositions after crystallization lie between equilibri.ım and fractional liquid lines of descent (Figure C1), while the steady state compositions after mixing lie on mixing ines between the end point on the liquid line of descent and the composition of the primitive magma entering the chamber (Figure C2). This is true for both major and trace elements.

This relationship to fractional and equilibrium liquid lines of descent means that closed system crysta:lization paths can be used to evaluate the range of compositions produced in a PRMC at steady-state. For example, Figure $\mathrm{C} 1$ shows the total calculated range in $\mathrm{FeO}$ and $\mathrm{Na}_{2} \mathrm{O}$ expected for liquids produced during crystallization in a steady-state periodically replenished magma chamber. The narrow width of this field down to low values of $\mathrm{MgO}$ shows that while this process may lead to complexity in the details of the fractionation trends, the effect is relatively small, and virtually negligible for high $\mathrm{MgO}$ compositions.

On the other hand, mixing along the liquid lines of descent can lead to compositions that differ significantly from the closed system path, particularly if there is mixing of a very high MgO magma with a very differentiated magma (Figure C2). This is possible in the "after mixing" step of the PRMC cycle. Although such mixtures have chemical compositions that differ substantially from the closed system LLD's, they also have recognizable chemical properties that are diagnostic. The mixed magma has higher $\mathrm{SiO}_{2}, \mathrm{FeO}, \mathrm{Na}_{2} \mathrm{O}, \mathrm{TiO}_{2}$ and incompatible trace elements, and lower $\mathrm{CaO}, \mathrm{Al}_{2} \mathrm{O}_{3}, \mathrm{CaO} / \mathrm{Al}_{2} \mathrm{O}_{3}$ and compatible trace elements than magma produced by closed system differentiation process. In order to create significant variability, however, mixing must occur between a very primitive and a very differentiated magma. Mixing between only a moderately evolved MORB and a typical MORB with 7$8 \% \mathrm{MgO}$, for example, will not produce large effects.

It is also important to note two characteristics of such mixed magmas. They have a single liquidus phase, olivine, and when 


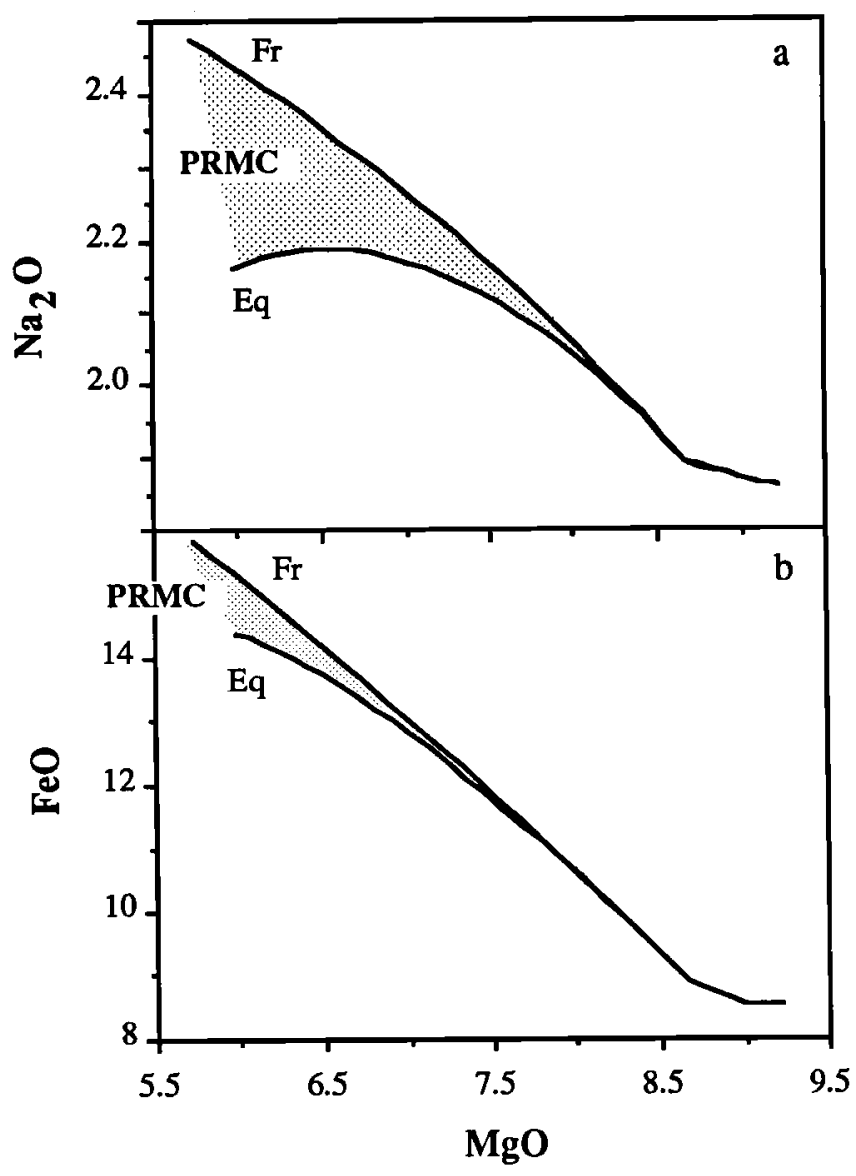

Fig. $\mathrm{Cl} . \mathrm{MgO}$ vs. $a) \mathrm{Na}_{2} \mathrm{O}$ and $b$ ) $\mathrm{FeO}$ for equilibrium and fractional Fig. C1. Mgover and Langmuir [1990] for a crystallization paths (LLios) It an be demonstrated [Langmuir, 1989] single starting compositon. It can be repished magma that the steady-state products of a periodically replenished magma chamber (PRMC) after the crystallization stage of the cycle lie between fractional and equilibrium crystallization, as indicated by the shading.

they cool they evolve quickly back towards the liquid line of descent of the parental magma. This is illustrated by LLD calculations in Figure C2. Therefore, to differ substantially from closed system evolution, these magmas must be caught immediately after mixing, prior to any subsequent cooling and differentiation. These characteristic properties are occasionally present in some MORB [see, e.g., Bender et al., 1984].

In conclusion, PRMC's lead to liquids that continually retum towards the closed system liquid line of descent. It is our intuition, based on the theory and calculations concerning PRMC's, that replenished magma chambers are not a dominant influence on the MORB chemical signal after fractionation correction. In the general case, the variations are quite similar to those produced by closed system crystallization. The one special case that can create significant variations produces specific compositional correlations and mineralogical characteristics that are recognizable, and not particularly common in our experience.
This is not at all to say, however, that mixing of magmas is not important. In fact, mixing of diverse magmas appears to be required to create the pooled melts that account for the global vector of compositional variation (see section 6.2).

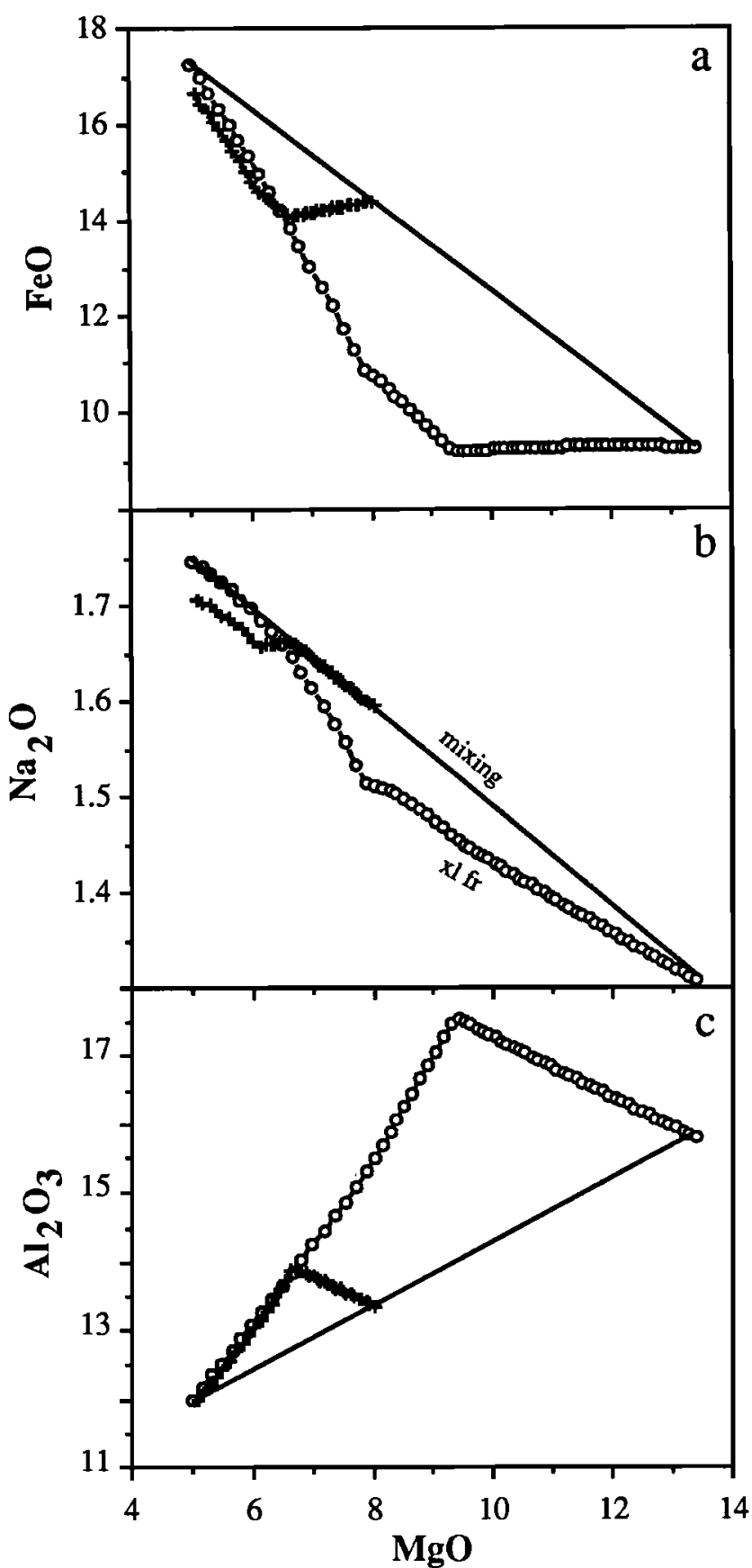

Fig. C2. $\mathrm{MgO}$ vs. $\mathrm{FeO}, \mathrm{Na}_{2} \mathrm{O}$ and $\mathrm{Al}_{2} \mathrm{O}_{3}$ for crystallization and mixing. The line formed of open circles ( $\mathrm{xl} \mathrm{fr}$ ) is a 1 atm LLD for a primitive basalt; the thin straight line is a mixing line between the primitive basalt and a derivative basalt with $5 \mathrm{wt} \% \mathrm{MgO}$; the line formed of the crosses shows the LLD for a mixed melt composition; all LLD's calculated using the method of Weaver and Langmuir [1990]. 


\section{C.2 In Situ Fractionation}

Another magma chamber process that may affect differentiation paths is in situ crystallization at the margins of a magma chamber. Magma chambers lose heat at their margins, and therefore solidify by crystallization in boundary layers along their margins. The magma in the interior of the chamber will evolve if residual melt from the boundary layer returns to the interior of the chamber and mixes with the magma that has not yet become part of the boundary solidification zone, a process that has become known as compositional convection [see, e.g., Sparks et al., 1984; Sparks, 1989]. The geochemical and petrological effects of this process have been considered by Langmuir [1989]. This form of differentiation differs from classical fractional crystallization because the evolution of the liquid in the chamber is controlled by addition of residual liquids from near the solidus rather than by the classical fractional crystallization which involves subtraction of minerals on the liquidus. The process of in situ crystallization differs from periodic replenishment because both mixing end-members evolve and change in composition, while for PRMC's, one endmember is both fixed and relatively primitive.

One of the characteristics of in situ crystallization is the removal of minerals that are not on the liquidus of the interior magma in the chamber. The composition of the returned liquid is controlled by minerals crystallizing at all levels in the boundary layer, ranging from liquidus to solidus. This means, for example, that a melt may show signs of clinopyroxene fractionation (decreasing $\mathrm{CaO}$ and $\mathrm{CaO} / \mathrm{Al}_{2} \mathrm{O}_{3}$ ), before clinopyroxene appears on the liquidus as a crystallizing phase.

Figure C3 illustrates this effect with an in situ fractionation trend where liquid is returned after $50 \%$ crystallization in a boundary layer. The normal trend for fractional crystallization

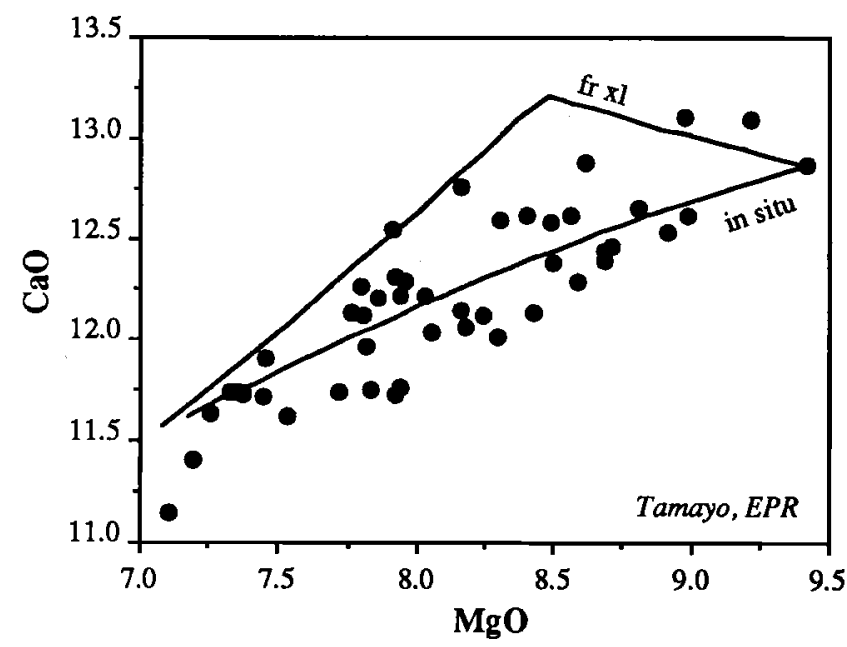

Fig. $\mathrm{C} 3 . \mathrm{CaO}$ vs. $\mathrm{MgO}$ for data from the Tamayo region of the East Pacific Rise (filled circles; Bender et al., 1984). Lines are calculated LLDs for simple fractional crystallization [Weaver and Langmuir, 1990 ], and in situ crystallization assuring retum of liquid after $50 \%$ crystallization in the boundary layer. does not show clinopyroxene crystallizing until the kink in the liquid line of descent at an $\mathrm{MgO}$ content of $8.5 \mathrm{wt} \%$. The in situ crystallization path, however, shows $\mathrm{CaO}$ decreasing immediately, due to retum of liquid from the boundary layer where clinopyroxene has crystallized. The in situ path appears to better reproduce the Tamayo data trend on this diagram.

The key parameter for the in situ model is the mean fraction of liquid that is returned to the chamber. For low mean melt fractions, the liquid line of descent may differ substantially from that of fractional crystallization. This can be understood from the simple reasoning using partition coefficients in section 2.2. When the melt fraction in the boundary layer becomes small, most elements reach their $1 / \mathrm{D}$ limit. Therefore moderately incompatible to compatible elements no longer change appreciably in concentration. Highly incompatible elements, with D's that approach zero, continue to increase in abundance. This means that the liquid returned to the magma chamber can be very enriched in incompatibles, and yet not have a particularly extreme major element composition. Thus in situ crystallization has the potential of creating large enrichments in incompatible elements, provided a large extent of crystallization in the boundary layer has occurred prior to return of the liquid to the chamber.

During melting of the mantle, clinopyroxene is the major mineral entering the melt up to about $20 \%$ melting, and for this reason it is often difficult to distinguish between melting, involving primarily the addition of clinopyroxene, and removal of clinopyroxene by crystallization. Of course, the F's involved are different and the two processes are likely to occur at markedly different pressures, but both processes produce the same general trends, including increases in $\mathrm{Na}_{2} \mathrm{O}$ and $\mathrm{FeO}$ and a decrease in $\mathrm{CaO} / \mathrm{Al}_{2} \mathrm{O}_{3}$, and therefore in a qualitative sense may be difficult to tell apart.

The mineral plagioclase, however, plays a critical role in identifying the effects of in situ crystallization. At low pressures plagioclase is a stable phase and its crystallization will impart a distinctive chemical signature to the liquid. Plagioclase is particularly important with respect to trace elements, because the element $\mathrm{Sr}$, incompatible during mantle melting processes, has a sufficiently high partition coefficient in plagioclase that its bulk distribution coefficient approaches 1 during crystallization. Therefore, any crystallization process involving plagioclase should be distinguishable from mantle melting processes. (Of course, plagioclase is also a possible mantle phase at very low pressures, but it melts out of the residue at low extents of melting, and appears not to play an important role as a residual mantle phase in MORB genesis. )

\section{C.3 High Pressure Fractionation}

While the evidence for low-pressure crystallization of MORB magmas is clear, some have argued that basaltic magmas also experience significant extents of high pressure fractionation prior to equilibration at low pressure. Proponents of ubiquitous high pressure fractionation cite as evidence the occurrence of highly magnesian pyroxenes in some ophiolite suites and occasionally in oceanic basalts [e.g., Elthon et al., 
1982; Elthon and Scarfe, 1984; c.f. Elthon, 1991], and the position of some MORB suites on pseudo-phase diagrams [e.g., Tormey et al., 1987].

Liquid line of descent programs that model crystallization at $1 \mathrm{~atm}$ have been successful due to the abundance of high quality experimental data at low pressure (see section 3.1). In theory, such liquid line of descent programs can be modified to model the effects of crystallization at high pressure; it is necessary only to know the pressure dependencies of the partition coefficients. Although both quality and quantity of experimental data at high pressure are much poorer than at 1 atm, the available data are nonetheless sufficient to begin quantitative evaluation of high pressure crystallization processes. We have incorporated pressure-dependencies of the $\mathrm{K}_{\mathrm{d}}$ 's for olivine, plagioclase and clinopyroxene into the liquid line of descent program of Weaver and Langmuir [1990; see Table $\mathrm{Cl}$ for $\mathrm{K}_{\mathrm{d}}$ functions used]. Due to the paucity of highpressure data, these results, although quantitative, are subject to greater uncertainties than the well-constrained calculations at $1 \mathrm{~atm}$.

Because of interlaboratory biases and the complexity of natural compositions, all LLD calculations are most reliable if they are tuned to the rocks under consideration. To evaluate the effects of high pressure crystallization, we have calibrated the LLD program to the FAMOUS compositions, which we will use as our test case for the high pressure crystallization model. The agreement of the calibration with high pressure experimental data on FAMOUS compositions is illustrated in Figure C4 where the phase appearances predicted by the high-pressure liquid line of descent program are compared with the experimentally determined phase appearances reported by Bender et al. [1978]. Note the overall consistency between the experimental and calculated appearances of olivine, plagioclase and clinopyroxene up to a pressure of $8 \mathrm{kbar}$. In detail, the calculated liquidus curves for olivine and plagioclase are $5.10^{\circ}$ lower than the experimentally determined liquidus

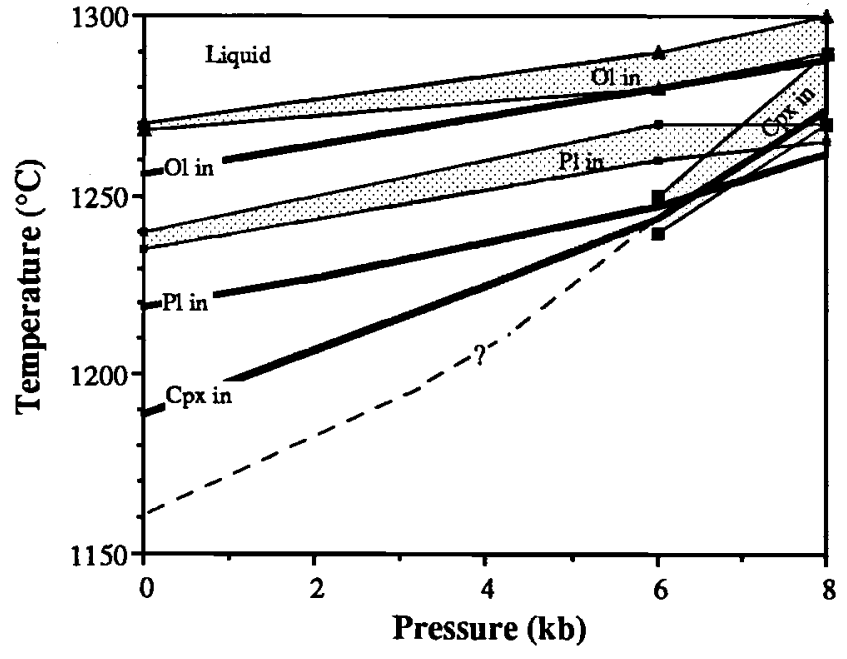

Fig. C4. Temperature vs. pressure diagram of phase appearances determined experimentally by Bender et al. [1978] for a sample from the FAMOUS region shown by the broad shaded bands representing the uncertainty in the experimental determinations. For example, the shaded band for plag-in lies between experiments in which there was no plagioclase, and experiments where plagioclase first appeared. The dashed line extending from the "clinopyroxene in" band at high pressure indicates great uncertainty in the position of this boundary due to nucleation problems in the low pressure experiments. Also shown (heavy solid lines) are the phase appearances calculated for the same melt composition using the liquid line of descent program of Weaver and Langmuir [1990], modified for crystallization at high pressure as described in the text.

curves. Nonetheless, at any one pressure, the experimentally determined and calculated temperature intervals for olivine + liquid stability are virtually identical. Although the appearance of clinopyroxene on the liquidus is not well constrained at low pressures in Bender et al.'s experimental data due to nucleation problems, the calculated appearance of clinopyroxene at $1 \mathrm{~atm}$ is in agreement with other experimental data for a similar

TABLE C1. Distribution Coefficient Equations with Pressure

\begin{tabular}{|c|c|c|}
\hline Phase & Component & Expression: $\log _{10} K_{d}=$ \\
\hline Olivine & $\begin{array}{l}\mathrm{MgO} \\
\mathrm{FeO}\end{array}$ & $\begin{array}{l}{\left[6921 / \mathrm{T}+3.4 \mathrm{Na}_{\mathrm{L}}+6.3 \mathrm{~K}_{\mathrm{L}}+0.00001154 \mathrm{P}-3.27\right] / 2.3} \\
\log _{10} \mathrm{~K}_{\mathrm{d}} \mathrm{Mg}-0.523\end{array}$ \\
\hline Plagioclase & $\begin{array}{l}\mathrm{CaAl}_{2} \mathrm{O}_{4} \\
\mathrm{NaAlO}_{2}\end{array}$ & $\begin{array}{l}2446 / T-1.122+0.2562 A n_{L} \\
{\left[3195+3283 A n_{L}+0.0506 \mathrm{P}\right] / T-\left[1.885 A n_{L}+2.3715\right]}\end{array}$ \\
\hline Clinopyroxene & $\begin{array}{l}\mathrm{MgO} \\
\mathrm{FeO} \\
\mathrm{CaSiO}_{3} \\
\mathrm{CaAl}_{2} \mathrm{O}_{4} \\
\mathrm{NaAlO}_{2} \\
\mathrm{TiO}_{2}\end{array}$ & $\begin{array}{l}{[3798+0.021 \mathrm{P}] / \mathrm{T}-2.28} \\
\log _{10} \mathrm{~K}_{\mathrm{d}} \mathrm{Mg}-0.6198 \\
{[1783+0.0038 \mathrm{P}] / \mathrm{T}-0.753} \\
{[2418+0.068 \mathrm{P}] / \mathrm{T}-2.3} \\
{[5087+0.073 \mathrm{P}] / \mathrm{T}-4.48} \\
{[1034+0.053 \mathrm{P}] / \mathrm{T}-1.27}\end{array}$ \\
\hline
\end{tabular}

$P$ in bars, $T$ in kelvins, compositional terms in mole fraction 
starting composition [Grove and Bryan, 1983]. Lastly, both the experimental and calculated data show a cross-over in the appearances of plagioclase and clinopyroxene on the liquidus between 6 and $7 \mathrm{kbar}$.

It is important to note that inconsistencies between the experimental data and the calculated liquid lines of descent do not necessarily imply inaccuracies in the calculated liquid line of descent. The liquid line of descent calculations are based on estimates of the distribution coefficients derived from numerous experiments and therefore minimize experimental problems, such as disequilibrium, that may have occurred in any one experiment. Furthermore, due to problems such as sodium or iron loss, the experimentally determined liquid lines of descent may not accurately reflect closed system fractionation, although the distribution coefficient determined from the experiment may accurately represent the partitioning between solid and the modified melt composition. Thus, a lessthan-perfect correspondence between experimental and calculated liquid lines of descent should not necessarily be attributed to problems with the calculation. On the other hand, the liquid line of descent program is as imperfect as its reproduction of the experimentally determined partition coefficients. For modeling of the subtle variations that exist within and between different magmatic suites, the bulk composition dependencies of the $K_{d}$ s become important, and caution should be exercised in using a generic liquid line of descent program to interpret such subtle variations.

\section{C.3.I General Results of High Pressure Fractionation}

As can be seen in the P-T diagram shown in Figure C4, the primary effect of crystallization at higher pressure is to increase the temperature of appearance of clinopyroxene
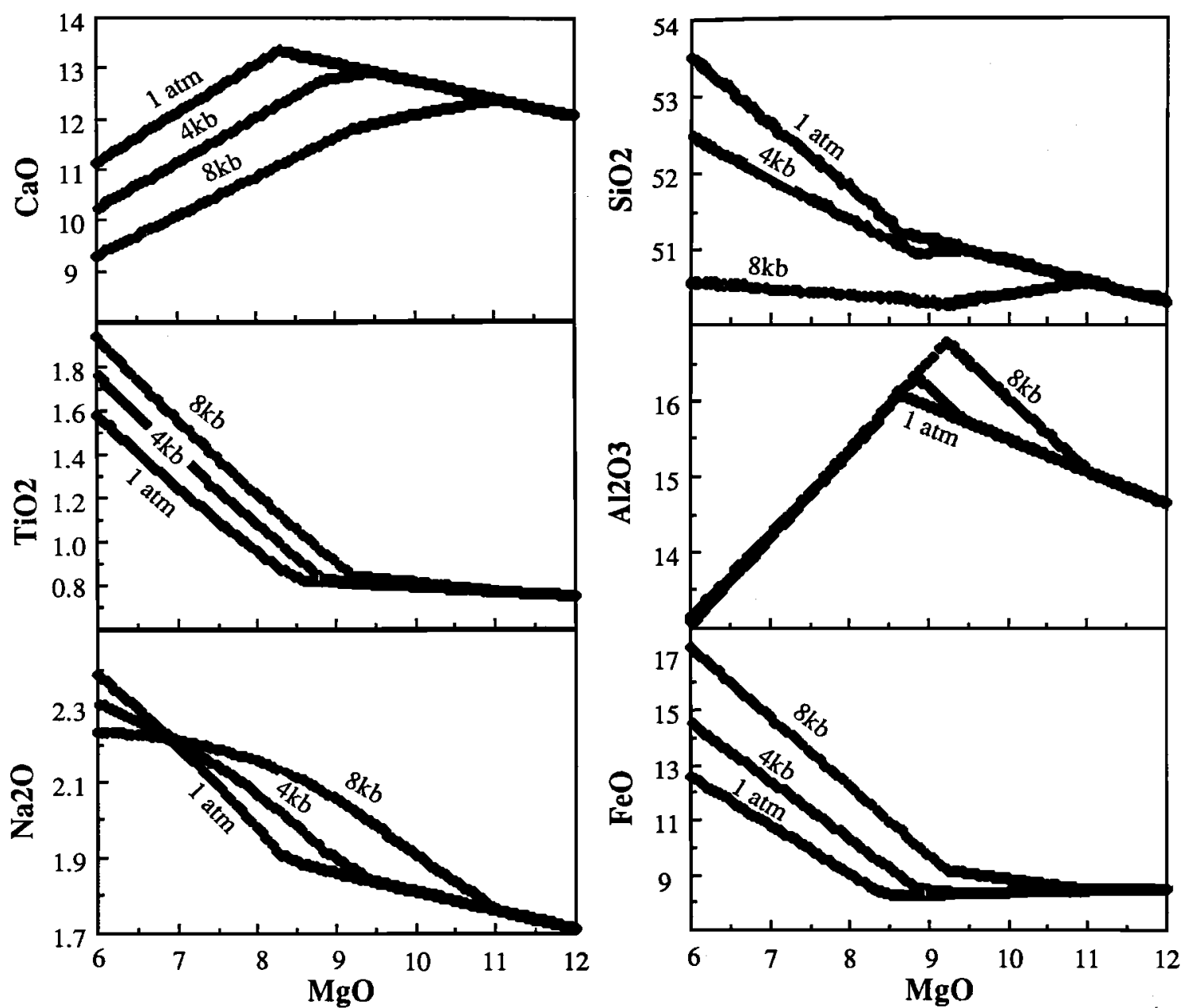

Fig. C5. Calculated liquid lines of descent fcr Model 1 crystallization at $1 \mathrm{~atm}, 4 \mathrm{kbar}$, and $8 \mathrm{kbar}$ of the melt composition presented in Table C2, using the program of Weaver and Langmuir [1990] modified for high-pressure crystallization as described in the text and shown in Table C.1. Compositions in figure are not exactly the same as those in the Table C2, because the compositions have leen corrected for interlaboratory bias so as to be comparable with the Melson data for the FAMOUS/AMAR region presented in Figures 22-23. These results are also shown in Figure 61 and compared to the FAMOUS/AMAR data. 
relative to olivine and plagioclase. In terms of compositional effects, liquid lines of descent on $\mathrm{MgO}$ variation diagrams show very different slopes when olivine crystallizes alone as compared to when plagioclase and clinopyroxene join the solid assemblage. Therefore the earlier appearance of these latter phases causes these changes of slope to sccur at higher $\mathrm{MgO}$ contents, which in turn causes the abundance of some oxides at $8 \mathrm{wt} \% \mathrm{MgO}$ to differ from those produced by differentiation at low pressure.

These effects are illustrated in Figure $\mathrm{C} 5$, where liquid lines of descent for a single high $\mathrm{MgO}$ parental composition are crystallized at different pressures. The starting composition, listed in Table C2 (starting "system" composition), was chosen by adding $2 \%$ olivine to a high- $\mathrm{MgO}$, low- $\mathrm{Na}$ sample composition from the FAMOUS/AMAR region. This composition was crystallized fractionally at pressures of $1 \mathrm{~atm}$, $4 \mathrm{kbar}$, and $8 \mathrm{kbar}$ (Figure C5) and the phase appearances and compositions are presented in Table $\mathrm{C} 2$. Relative to differentiation at $1 \mathrm{~atm}$, at $8 \mathrm{wt} \% \mathrm{MgO} \approx$ higher pressure of crystallization usually causes $\mathrm{Na}_{2} \mathrm{O}, \mathrm{FeO}, \mathrm{TiO}_{2}$ and $\mathrm{K}_{2} \mathrm{O}$ to be higher, and $\mathrm{CaO}, \mathrm{Al}_{2} \mathrm{O}_{3}$ and $\mathrm{SiO}_{2}$ to be lower. An important additional effect is that at higher presi,ure the crystallizing plagioclase is of a more sodic compositicn [e.g., Bender et al., 1978; Fram and Longhi, 1991], and thercfore the $8 \mathrm{kbar}$ liquid line of descent shows a marked leveling-oif of $\mathrm{Na}_{2} \mathrm{O}$ enrichment as fractionation proceeds.

\section{C.3.2 Models for High Pressure Fractionation}

There are numerous possible high-pressure crystallization scenarios. Consider first a fractionation model in which there is a single parental magma composition, different batches of which segregate from the mantle and fractionate at different pressures, followed by immediate transport to the surface and eruption. This is Model 1, and would lead to the results described above and shown in Figure C5 and Table C2.

In another possible model (Model 2), different batches of the same parental magma composition fractionate at some high pressure, but the various batches fractionate to different extents prior to transport to the surface, where they fractionate further to $8 \mathrm{wt} \% \mathrm{MgO}$, and erupt. For Model 2, we used the $8 \mathrm{kbar}$ liquid line of descent for the high pressure stage of crystallization, and at various stages of crystallization at 8 kbar, the residual liquid composition was "removed" and fractionated at $1 \mathrm{~atm}$. The results of this exercise are shown in Figure C6. Differences in the fracticnating assemblages between $1 \mathrm{~atm}$ and $8 \mathrm{kbar}$ are evident. For example, on the plot of $\mathrm{MgO}$ vs. $\mathrm{CaO}$, it is clear that at $8 \mathrm{kbar}$, clinopyroxene and olivine are stable at $>10 \mathrm{wt} \% \mathrm{MgO}$, leading to decreasing $\mathrm{CaO}$ with decreasing $\mathrm{MgO}$; in contrast, the same magma composition ( $>10 \mathrm{wt} \% \mathrm{MgO}$ ) at 1 atm shows increasing $\mathrm{CaO}$ with decreasing $\mathrm{MgO}$ due to the crystallization of olivine alone. The range in compositions produced by Model 2 is bracketed by the 1 atm and 8 kbar LLDs, as it was for Model 1,

TABLE C2. Model 1

\begin{tabular}{|c|c|c|c|c|c|c|c|c|c|c|c|c|c|c|c|c|c|}
\hline \multicolumn{6}{|c|}{ Pressure $=1 \mathrm{~atm}$} & \multicolumn{6}{|c|}{ Pressure $=4 \mathrm{kbar}$} & \multicolumn{6}{|c|}{ Pressure $=8 \mathrm{kbar}$} \\
\hline fraction: & System & $\begin{array}{l}\text { Liquid } \\
0.9993\end{array}$ & Plag & $\begin{array}{l}\text { Olivine } \\
0.0007\end{array}$ & $C P X$ & fraction: & System & $\begin{array}{l}\text { Liquid } \\
0.9999\end{array}$ & Plag & $\begin{array}{c}\text { Olivine } \\
0.0001\end{array}$ & $C P X$ & fraction: & System & $\begin{array}{l}\text { Liquid } \\
0.9989\end{array}$ & Plag & $\begin{array}{c}\text { Olivine } \\
0.0011\end{array}$ & $C P X$ \\
\hline $\mathrm{SiO} 2$ & 50.00 & 50.01 & & 40.86 & & $\mathrm{SiO} 2$ & 50.00 & 50.00 & & 40.86 & & $\mathrm{SiO}_{2}$ & 50.00 & 50.01 & & 40.85 & \\
\hline $\mathrm{TiO} 2$ & 0.75 & 0.75 & & 0.00 & & $\mathrm{TiO} 2$ & 0.75 & 0.75 & & 0.00 & & $\mathrm{TiO} 2$ & 0.75 & 0.75 & & 0.00 & \\
\hline $\mathrm{A} 12 \mathrm{O} 3$ & 14.65 & 14.66 & & 0.00 & & $\mathrm{AL} 2 \mathrm{O} 3$ & 14.65 & 14.65 & & 0.00 & & $\mathrm{~A}: 2 \mathrm{O} 3$ & 14.65 & 14.66 & & 0.00 & \\
\hline $\mathrm{FeO}$ & 8.30 & 8.30 & & 9.84 & & $\mathrm{FeO}$ & 8.30 & 8.30 & & 9.83 & & $\mathrm{FeO}$ & 8.30 & 8.30 & & 9.85 & \\
\hline $\mathrm{MgO}$ & 12.50 & 12.48 & & 49.31 & & $\mathrm{MgO}$ & 12.50 & 12.50 & & 49.32 & & $\mathrm{MgO}$ & 12.50 & 12.46 & & 49.30 & \\
\hline $\mathrm{CaO}$ & 12.05 & 12.06 & & 0.00 & & $\mathrm{CaO}$ & 12.05 & 12.05 & & 0.00 & & $\mathrm{CsO}$ & 12.05 & 12.06 & & 0.00 & \\
\hline $\mathrm{Na} 2 \mathrm{O}$ & 1.75 & 1.75 & & 0.00 & & $\mathrm{Na2O}$ & 1.75 & 1.75 & & 0.00 & & $\mathrm{Na2O}$ & 1.75 & 1.75 & & 0.00 & \\
\hline \multirow[t]{2}{*}{$T E M P=$} & 1291 & \multicolumn{3}{|c|}{ Frac. Liquid Remaining: } & 0.9993 & TEMP $=$ & 1308 & \multicolumn{3}{|c|}{ Frac. Liquid Remaining: } & 0.9999 & $T E M P=$ & 1324 & \multicolumn{3}{|c|}{ Frac. Liguid Remaining: } & 0.9989 \\
\hline & System & $\begin{array}{l}\text { Liquid } \\
0.9919\end{array}$ & Plag & $\begin{array}{c}\text { Olivine } \\
0.0027\end{array}$ & $C P X$ & & System & $\begin{array}{l}\text { Liquid } \\
0.9989\end{array}$ & Plag & Olivine & $\begin{array}{l}C P X \\
0,0001\end{array}$ & & System & Liquid & Plag & Olivine & $\begin{array}{ll}C P X \\
0 \times 032\end{array}$ \\
\hline $\begin{array}{l}\text { fraction: } \\
\text { SiO2 }\end{array}$ & 50.93 & $\begin{array}{r}0.9919 \\
50.97\end{array}$ & $\begin{array}{r}0.0054 \\
47.68\end{array}$ & $\begin{array}{r}0.0027 \\
40.32\end{array}$ & & $\begin{array}{l}\text { fraction: } \\
\mathrm{SiO} 2\end{array}$ & 50.67 & $\begin{array}{r}0.9989 \\
50.68\end{array}$ & & $\begin{array}{l}0.001 \\
40.49\end{array}$ & $\begin{array}{r}0.0001 \\
53.17\end{array}$ & $\begin{array}{l}\text { raction: } \\
\mathrm{SiO} 2\end{array}$ & 50.25 & $\begin{array}{r}0.9963 \\
5 \quad 50.25\end{array}$ & & $\begin{array}{r}0.0005 \\
40.72\end{array}$ & $\begin{array}{r}0.0032 \\
52.94\end{array}$ \\
\hline $\mathrm{TiO} 2$ & 0.82 & 0.83 & 0.00 & 0.00 & & $\mathrm{TiO} 2$ & 0.80 & 0.81 & & 0.00 & 0.29 & TiO2 & 0.77 & 0.77 & & 0.00 & 0.36 \\
\hline $\mathrm{A} 12 \mathrm{O} 3$ & 16.10 & 16.04 & 33.63 & 0.00 & & $\mathrm{~A} 1203$ & 15.71 & 15.73 & & 0.00 & 4.25 & $\mathrm{Al} 2 \mathrm{O} 3$ & 15.05 & 15.09 & & 0.00 & 5.16 \\
\hline FeO & 8.02 & 8.05 & 0.00 & 12.72 & & $\mathrm{~F} \infty \mathrm{O}$ & 8.12 & 8.12 & & 11.80 & 3.84 & $\mathrm{FeO}$ & 8.25 & 8.26 & & 10.54 & 3.54 \\
\hline $\mathrm{MgO}$ & 8.97 & 8.92 & 0.00 & 46.97 & & $\mathrm{MgO}$ & 9.89 & 9.85 & & 47.71 & 19.42 & $\mathrm{MgO}$ & 11.50 & 11.46 & & 48.73 & 20.48 \\
\hline $\mathrm{CaO}$ & 13.24 & 13.25 & 16.61 & 0.00 & & $\mathrm{CaO}$ & 12.92 & 12.93 & & 0.00 & 18.79 & $\mathrm{CaO}$ & 12.38 & 12.37 & & 0.00 & 17.27 \\
\hline $\mathrm{Na} 2 \mathrm{O}$ & 1.92 & 1.93 & 2.08 & 0.00 & & $\mathrm{Na2O}$ & 1.88 & 1.88 & & 0.00 & 0.23 & $\mathrm{Na} 2 \mathrm{O}$ & 1.80 & 1.80 & & 0.00 & 0.25 \\
\hline \multirow[t]{2}{*}{$T E M P=$} & 1199 & \multicolumn{3}{|c|}{ Frac. Liquid Remaining: } & 0.8929 & TEMP $=$ & 1240 & \multicolumn{3}{|c|}{ Frac. Liquid Remaining: } & 0.9238 & TEMP $=$ & 1299 & \multicolumn{3}{|c|}{ Frac. Liquid Remaining: } & 0.9668 \\
\hline & System & Liquid & Plag & Olivine & $C P X$ & & System & Liquid & Plag & Olivine & $C P X$ & & System & Liquid & Plag & Olivine & $C P X$ \\
\hline fraction: & & 0.9813 & 0.0102 & 0.0028 & 0.0057 & fraction: & & 0.9745 & 0.0132 & 0.003 & 0.0092 & fraction: & & 0.9948 & 0.0008 & 0.0002 & 0.0042 \\
\hline $\mathrm{SiO} 2$ & 51.25 & 51.30 & 47.92 & 40.16 & 53.39 & $\mathrm{SiO}_{2}$ & 50.64 & 50.67 & 48.68 & 40.28 & 52.95 & $\mathrm{SiO} 2$ & 49.96 & 49.95 & 49.66 & 40.25 & 52.20 \\
\hline $\mathrm{TiO} 2$ & 0.87 & 0.89 & 0.00 & 0.00 & 0.25 & $\mathrm{TiO}_{2}$ & 0.83 & 0.85 & 0.00 & 0.00 & 0.32 & $\mathrm{TiO} 2$ & 0.84 & 0.85 & 0.00 & 0.00 & 0.42 \\
\hline $\mathrm{A} 12 \mathrm{O} 3$ & 15.66 & 15.59 & 33.47 & 0.00 & 3.15 & $\mathrm{Al} 2 \mathrm{O} 3$ & 16.31 & 16.24 & 32.96 & 0.00 & 4.62 & A.12O3 & 16.75 & 16.78 & 32.29 & 0.00 & 6.54 \\
\hline $\mathrm{F}=\mathrm{O}$ & 8.27 & 8.36 & 0.00 & 13.52 & 4.20 & $\mathrm{FeO}$ & 8.28 & 8.41 & 0.00 & 12.92 & 4.26 & $\mathrm{FeO}$ & 8.93 & 8.95 & 0.00 & 13.06 & 4.54 \\
\hline $\mathrm{MgO}$ & 8.65 & 8.59 & 0.00 & 46.31 & 17.97 & $\mathrm{MgO}$ & 9.22 & 9.14 & 0.00 & 46.80 & 19.31 & $\mathrm{MgO}$ & 9.64 & 9.60 & 0.00 & 46.69 & 20.29 \\
\hline $\mathrm{CaO}$ & 13.34 & 13.30 & 16.43 & 0.00 & 20.85 & $\mathrm{CaO}$ & 12.76 & 12.70 & 15.83 & 0.00 & 18.28 & $\mathrm{CaO}$ & 11.82 & 11.80 & 15.06 & 0.00 & 15.65 \\
\hline $\mathrm{Na} 20$ & 1.95 & 1.96 & 2.19 & 0.00 & 0.20 & $\mathrm{Na} 2 \mathrm{O}$ & 1.96 & 1.97 & 2.54 & 0.00 & 0.26 & $\mathrm{Na} 2 \mathrm{O}$ & 2.07 & 2.07 & 2.99 & 0.00 & 0.36 \\
\hline$T E M P=$ & 1192 & Frac. Liqu & id Remair & ining: & 0.8314 & TEMP $=$ & 1223 & Frac. Liqu & uid Remai & & 0.8562 & $T E M P=$ & 1256 & Frac. Liqu & Remair & & 0.8162 \\
\hline
\end{tabular}



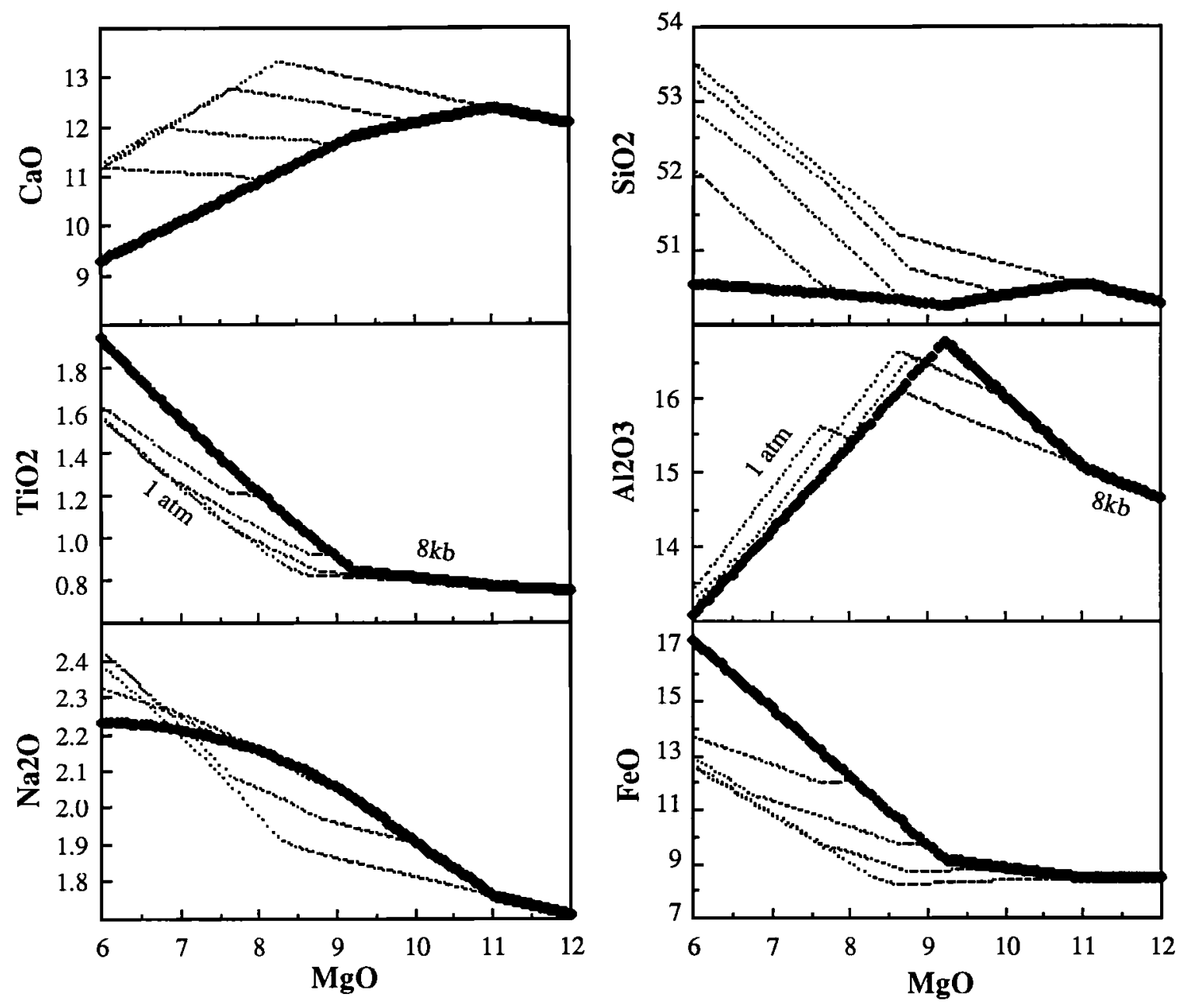

Fig. C6. Calculated liquid lines of descent for fractional crystallization of the parental magma composition shown in Table C2, using the liquid line of descent program of Weaver and Langmuir [1990], modified for high-pressure fractionation as described in the text and shown in Table C1. In this model (Model 2), crystallization begins at 8 kbar (solid curves), and at different stages of differentiation (different values of $\mathrm{MgO}$ ) batches of this evolving magma are transported to low pressure where they fractionate at $1 \mathrm{~atm}$ (dotted curves).

and therefore the total range in any one oxide is virtually the same as that produced by Model 1 above.

Other fractionation models for high pressure differentiation can be envisioned. For example, one could consider the case of polybaric fractionation in which a particular parental magma composition begins crystallizing at high pressure, and as it migrates toward the surface it fractionates at successively lower pressures. This model, however, produces only a single composition at the surface, rather than producing the whole range of compositions at $8 \mathrm{wt} \% \mathrm{MgO}$ such as would be necessary to account for the Atlantic-type local vector.

The results shown in Figure $\mathrm{C5}$ and $\mathrm{C} 6$ are based on experiments that investigated basalts from the FAMOUS area, and are primarily applicable to examining the effects of high pressure fractionation in samples of this parental magma composition. We have also examined, however, the available experimental data for a starting composition from the Kane region, which differs from the FAMOUS composition in having higher $\mathrm{Na}_{2} \mathrm{O}$ and $\mathrm{SiO}_{2}$ and lower $\mathrm{FeO}$. There are some differences in the pressure-dependencies derived from experiments for the two starting compositions, but there is agreement for the two different compositions in the overall trends of the effects of high pressure crystallization. Thus these calculations are able to give a reasonable estimate of the importance of high pressure fractionation for explaining the global and local MORB systematics (discussed in sections 6.2 and 6.3).

Acknowledgements. This paper results from over a decade of ongoing work, to which dozens of people have contributed in important ways, particularly John Bender. The immediate manuscript has benefited from numerous interactions at the short course and reviews by or discussion with Rodey Batiza, Donna Blackman, Dana Desonie, 
Bob Detrick, Kevin Johnson, Jason Phipps Mcrzan, Yao Ling Niu, Jennifer Reynolds and Katrin Wiese. Discussions with Ro Kinzler and Tim Grove provided impetus to investigate the role of high pressure fractionation thoroughly, and to address compositional dependence of the olivine partition coefficients. Thorough formal reviews by and discussion with Dave Christie, Jean-Guy Schilling and John Sinton aided very much in revising the manuscript. Charlene Straub worked long hours far beyond the call of duty in editing the manuscript and preparing it for publication. Joan Totten helped with the final preparation of the manuscript. Patty Catanzaro drafted many of the figures. This work has been supported by numerous grants from the National Science Foundation, particularly OCE 90-14925 (CHL) and OCE 90-16317 (EMK).

\section{REFERENCES}

Agee, C. B., and D. Walker, Aluminum partitioning between olivine and ultrabasic silicate liquid to $6 \mathrm{GPa}$, Contrib. Mineral. Petrol., $105,243-254,1990$.

Ahern, J. L., and D. L. Turcotte, Magma migration beneath an ocean ridge, Earth Planet. Sci. Lett., 45, 115-122, 1979.

Albarede, F., How deep do common basaltic magmas form and differentiate?, J. Geophys. Res., 97, 10,997-11,009, 1992

Albarede, F., and V. Tamagnan, Modelling the recent geochemical evolution of the Piton de la Foumaise volcano, Reunion Island, 1931-1986, J. Petrol., 29, 997-1030, 1988.

Allegre, C. J. and Y. Bottinga, Tholeiite, Alkali Basalt and Ascent Velocity, Nature, 252, 31-31, 1974

Allegre, C. J., and J. F. Minster, Quantitative models of trace element behavior in magmatic processes, Earth Planet. Sci. Lett., 38, 1-25, 1978.

Allegre, C. J., and D. L. Turcotte, Implications of a two-component marble-cake mantle, Nature, 323, 123-127, 1986.

Allegre, C. J., B. Hamelin, and B. Dupre, Statistical analysis of isotopic ratios in MORB: the mantle blob cluster model and the convective regime of the mantle, Earth Planet. Sci. Lett., 71, 71-84, 1984.

Bass, M. N., Variable abyssal basalt populations and their relation to sea-floor spreading rates, Earth Planet. Sci. Lett., 11, 18-22, 1971.

Batiza, R., Inverse relationship between $\mathrm{Sr}$ isotope diversity and rate of oceanic volcanism has implications for mantle heterogeneity, Nature, 309, 440-441, 1984.

Batiza, R., and D. Vanko, Petrology of young Pacific seamounts, $J$. Geophys. Res., 89, 11,235-11,260, 1984.

Batiza, R., W. G. Melson, and T. O'Heam, Simple magma supply geometry inferred beneath a segment of the Mid-Atlantic Ridge. Nature, 335, 428-431, 1988.

Batiza, R., Y. Niu, and W. C. Zayac, Chemistry of seamounts near the East-Pacific Rise: Implications for the geometry of sub-axial mantle flow, Geology, 18, 1122-1125, 1990.

Beatty, P., C. Ford, and D. Russel, Partition coefficients for olivinemelt and orthopyroxene-melt systems, Contrib. Mineral. Petrol., 109, 212-224, 1991

Bender, J. F., F. N. Hodges, and A. E. Bence, Petrogenesis of basalts from the project FAMOUS area: experimental study from 0 to 15 kbars, Earth Planet. Sci. Lett., 41, 277-302, 1978.

Bender, J. F., C. H. Langmuir, and G. N. Hanson, Petrogenesis of basalt glasses from the Tamayo region, East Pacific Rise, J. Petrol., 25, 213-254, 1984 .

Bottinga, Y. and C. J. Allegre, Thermal aspects of sea-floor spreading and the nature of the oceanic crust, Tectonophys. 18, 1-17, 1973.

Bougault, H., and M. Treuil, Mid-Atlantic Ridge: Zero-age geochemical variations between Azores and $22^{\circ} \mathrm{N}$, Nature, 286, 209-212, 1980

Brodholt, J. P., and R. Batiza, Global systematics of unaveraged MORB compositions: Comment on "Global correlations of ocean ridge basalt chemistry with axial depth and crustal thickness" by $\mathrm{E}$. M. Klein and C. H. Langmuir, J. Geophys. Res., 94, 4231-4240, 1989.

Bryan, W. B., and J. G. Moore, Compositional variations of young basalts in the Mid-Atlantic Ridge rift valley near lat. $36^{\circ} 49^{\prime} \mathrm{N}$, Geol.
Soc. Am. Bull., 88, 556-570, 1977.

Bryan, W. B., and H. J. B. Dick, Contrasted abyssal basalt liquidus trends: Evidence for mantle major element heterogeneity, Earth Planet. Sci. Lett., 58, 15-26, 1982.

Buck, W. R. and W. Su, Focussed mantle upwelling below mid-ocean ridges due to feedback between viscosity and melting, Geophys. Res. Lett., 16, 641-644, 1989.

Bunch, A. W. H., and B. L. N. Kennett, The crustal structure of the Reykjanes Ridge at $59^{\circ} 30^{\prime} \mathrm{N}$, Geophys. J. R. Astron. Soc., 61, 141$166,1980$.

Byerly, G. R., W. G. Melson, and P. R. Vogt, Rhyodacites, andesites, ferro-basalts and ocean tholeiites from the Galapagos spreading center, Earth Planet. Sci. Lett., 30, 215-221, 1976.

Carmichael, I. S. E., F. J. Tumer, and J. Verhoogen, Igneous Petrology, 739 pp., McGraw-Hill, New York, 1974.

Castillo, P. R., E. Klein, S. B. Shirey, J. F. Bender, R. Batiza, and C. Langmuir, Relationship of $\mathrm{Sr}-\mathrm{Nd}-\mathrm{Pb}$ isotopes of EPR and MAR basalt glasses to bathymetry and ridge segmentation, EOS, 69 , $1477,1988$.

Cawthom, R. G., Degrees of melting in mantle diapirs and the origin of ultrabasic liquids, Earth Planet. Sci. Lett., 27, 113-120, 1975.

Clague, D. A., F. A. Frey, G. Thompson, and S. Rindge, Minor and trace element geochemistry of volcanic rocks dredged from the Galapagos Spreading Center: Role of crystal fractionation and mantle heterogeneity, J. Geophys. Res., 86, 9469-9482, 1981.

Cohen, R. S., and R. K. O'Nions, The lead, neodymium and strontium isotopic structure of ocean ridge basalts, J. Petrol., 23, 229-324, 1982.

Condomines, M., C. H. Hemond, and C. J. Allegre, U-Th-Ra radioactive disequilibria and magmatic processes, Earth Planet. Sci. Lett., 90, 243-262, 1988.

Dick, H. J. B., Abyssal peridotites, very slow spreading ridges, and ocean ridge magmatism, in Magmatism in the Ocean Basins, edited by A. D. Saunders and M. J. Norry, pp. 71-105, Geol. Soc. London Spec. Pub. No. 42, 1989.

Dick, H. J. B., R. L. Fisher, and W. B. Bryan, Mineralogic variability of the uppermost mantle along mid-ocean ridges, Earth Planet. Sci. Lett., 69, 88-106, 1984.

Dixon, J. E., E. Stolper, and J. R. Delaney, Infrared spectroscopic measurements of $\mathrm{CO}_{2}$ and $\mathrm{H}_{2} \mathrm{O}$ in Juan de Fuca basaltic glasses, Earth Planet. Sci. Lett., 90, 87-104, 1988.

Dmitriev, L.V., A. V. Sobolev, A. V. Uchanov, T. V. Malysheva, and W. G. Melson, Primary differences in oxygen fugacity and depth of melting in the mantle source regions for oceanic basalts, Earth Planet. Sci. Lett., 70, 303-310, 1984

Dungan, M. A., and J. M. Rhodes, Residual glasses and melt inclusions in basalts from DSDP legs 45 and 46: Evidence for magma mixing, Contrib. Mineral Petrol., 67, 417-431, 1978.

Dupre, B., B. Lambret, D. Rousseau, and C. J. Allegre, Limitations on the scale of mantle heterogeneities under ocean ridges, Nature, 294, $552-554,1981$

Elliott, T. R., C. J. Hawkesworth, and K. Gronvold, Dynamic melting of the Iceland plume, Nature, 351, 201-206, 1991.

Elthon, D., Pressure of origin of primary mid-ocean ridge basalts, Magmatism in the Ocean Basins, edited by A. D. Sounders and M. J. Norry, pp. 125, Geol. Soc. Spec. Publ. London 42, 1989.

Elthon, D., and C. M. Scarfe, High-pressure phase equilibria of a highmagnesia basalt and the genesis of primary oceanic basalts, Am. Mineral., 69, 1-15, 1984.

Elthon, D., J. F. Casey, and S. Komor, Mineral chemistry of ultramafic cumulates for the North Arm Mountain massif of the Bay of Islands ophiolite: evidence for high pressure crystal fractionation of oceanic basalts, J. Geophys. Res., 87, 8717-8734, 1982.

Etoubleau, J., O. Corre, J. L. Joron, H. Bougault, and M. Treuil, Costa Rica Rift: variably depleted basalts in the same hole. In Init. Repts. DSDP 69 (eds. J. R. Cann et al.) pp. 765-773, 1983.

Evans, J. R., and Sacks, I. S., Deep structure of the Iceland plateau, $J$. Geophys. Res., 84, 6859-6866, 1979.

Falloon, T. J., and D. H. Green, Anhydrous partial melting of MORB pyrolite and other peridotite compositions at $10 \mathrm{~kb}$ : Implications 
for the origin of primitive MORB glasses, Mineral. and Petrol., 37, $181-219,1987$.

Falloon, T. J. and D. H. Green, Anhydrous partial melting of peridotite from 8 to $35 \mathrm{~kb}$ and the petrogenesis of MORB, J. Petrol. $S p$. Lithosphere Issue, 379-414, 1988.

Falloon, T. J., D. H. Green, C. J. Hatton, and K. L. Harris, Anhydrous partial melting of a fertile and depleted peridotite from 2 to $30 \mathrm{~kb}$ and application to basalt petrogenesis, J. Petrol., 29, 1257-1282, 1988.

Feigenson, M. D., A. W. Hofmann, and F. J. Spera, Case studies on the origin of basalt $\amalg$. The transition from tholeiitic to alkalic volcanism on Kohala volcano, Hawaii, Contrib. Mineral. Petrol. $84,390-405,1983$.

Fisk, M. R., J. G. Schilling, and H. Sigurdsson, An experimental investigation of Iceland and Reykjanes Ridge tholeiites, I. Phase relations, Contrib. Mineral. Petrol., 74, 316-374, 1980.

Fisk, M. R., A. E. Bence, and J.-G. Schilling, Major element chemistry of Galapagos Rift Zone magmas and their phenocrysts, Earth Planet. Sci. Lett., 61, 171-189, 1982

Ford, C. E., D. G. Russell, J. A. Craven, and M. R. Fisk, Olivine-liquid equilibria: temperature, pressure and composition dependence of the crystal/liquid cation partition coefficients for $\mathrm{Mg}, \mathrm{Fe} 2+, \mathrm{Ca}$ and $\mathrm{Mn}$, $J$. Petrol, 24, 256-265, 1983.

Fomari, D. J., M. R. Perfit, A. Malahoff, and R. Embley, Geochemical studies of abyssal lavas recovered by DSRV Alvin from Eastern Galapagos Rift, Inca Transform and Ecuador Rift. 1. Major element variations in natural glasses and spatial distribution of lavas, $J$. Geophys. Res., 88, 10,519-10,529, 1983.

Fox, P. J., and D. G. Gallo, A tectonic model for ridge-transform-ridge plate boundaries: implications for the structure of oceanic lithosphere, Tectonophysics, 104, 205-242, 1984.

Fram, M. S., and J. Longhi, Plagioclase/melt partitioning as a function of pressure, EOS, 72, 304, 1991

Frey, F. A., C.-Y. Suen, and H. W. Stockman, The Ronda high temperature peridotite: Geochemistry and petrogenesis, Geochim. Cosmochim. Acta, 49, 2469-2491, 1985.

Frey, F. A., W. S. Wise, M. O. Garcia, H. West, S.-T. Kwon, and A. Kennedy, Evolution of Mauna Kea volcano, Hawaii: Petrologic and geochemical constraints on postshicld volcanism, J. Geophys. Res., 95, 1271-1300, 1990.

Fryer, P., J. M. Sinton, and J. A. Philpotts, Basaltic glasses from the Mariana Trough, Initial Rep. Deep Sea Drill. Proj., 60, 601-609, 1981.

Fryer, P., B. Taylor, C. H. Langmuir, and A. G. Hochstaedter, Petrology and geochemistry of lavas from the Sumisu and Torishima backarc rifts, Earth and Planet. Sci. Lett., 100, 161-178, 1990.

Fujii, T., and C. M. Scarfe, Composition of liquids coexisting with spinel lherzolite at $10 \mathrm{kbar}$ and the genesis of MORBs, Contrib. Mineral. Petrol., 90, 18-28, 1985.

Galer, S. J. G., and R. K. O'Nions, Magmagenesis and the mapping of chemical and isotopic variations in the mantle, Chem. Geol., 56, 45-61, 1986.

Gast, P. W., Trace element fractionation and the origin of tholeiitic and alkaline magma types, Geochim. Cosmochim. Acta, 32, 1057 1086, 1968.

Ghiorso, M., I. S. Carmichael, M. L. Rivers, and R. O. Sack, The Gibbs Free Energy of mixing of natural silicate liquids; an expanded regular solution approximation for the calculation of magmatic intensive variables, Contrib. Mineral. Petrol., 84, 107-145, 1983.

Goldstein, S. J., M. T. Murrell, and D. R. Janecky, Th and U isotopic systematics of basalts from the Juan de Fuca and Gorda Ridges by mass spectrometry, Earth Planet. Sci. Lett., 96, 134-146, 1989.

Goldstein, S. J., M. T. Murrell, D. R. Janecky, J. R. Delaney, and D. A. Clague, Geochronology and petrogenesis of MORB from the Juan de Fuca and Gorda Ridges by ${ }^{238} \mathrm{U}-{ }^{230} \mathrm{Th}$ disequilibrium, Earth Plant. Sci. Lett., 107, 25-41, 1991. (Erratum, 109, 255-272, 1992)

Green, D. H., Composition of basaltic magmas as indicators of conditions of origin: application to oceanic volcanism, Phil. Trans. Roy. Soc. Lond., 268, 707-725, 1971.

Green, D. H., and A. E. Ringwood, The genesis of basaltic magmas, Contrib. Mineral. Petrol., 15, 103-190, 1967.
Grove, T. L., and W. B. Bryan, Fractionation of pyroxene-phyric MORB at low pressure: an experimental study, Contrib. Mineral. Petrol., 84, 293-309, 1983.

Grove, T. L., D. C. Gerlach, and T. W. Sando, Origin of calc-alkaline series lavas at Medicine Lake volcano by fractionation, assimilation and mixing, Contrib. Mineral. Petrol., 80, 160-182, 1982.

Grove, T. L., R. J. Kinzler, and W. B. Bryan, Natural and experimental phase relations of lavas from Serocki volcano, Proc. ODP, Sci. Results, 106/109, 9-17, 1990

Grover, J. E., D. H. Lindsley, and A. E. Bence, Experimental phase relations of olivine vitrophyres from breccia 14321: the temperature- and pressure-dependence of $\mathrm{Fe}-\mathrm{Mg}$ partitioning for olivine and liquid in a highlands melt-rock, Proc. Lunar Plant. Sci. Conf., 11, 179-196, 1980.

Hamelin, B., B. Dupre, and C. J. Allegre, Lead-strontium isotopic variations along the East Pacific Rise and the Mid-Atlantic Ridge: a comparative study, Earth Planet. Sci. Lett., 67, 340-350, 1984.

Hanan, B. B., R. H. Kingsley, and J. G. Schilling, Pb isotope evidence in the south Atlantic for migrating ridge-hotspot interactions, Nature, 322, 137-144, 1986

Hanson, G. N., Evolution of the suboceanic mantle, Geol. Soc. London, 134, 235-253, 1977.

Hanson, G. N., and C. H. Langmuir, Modelling of major elements in mantle systems using trace element approaches, Geochim. Cosmochim. Acta, 42, 725-741, 1978.

Hart, S. R., and A. Zindler, In search of bulk Earth composition, Chem. Geol., S7, 247-267, 1986.

Hekinian, R., and G. Thompson, Comparative geochemistry of volcanics from rift valleys, transforms and aseismic ridges, Contrib. Mineral. Petrol., 57, 145-62, 1976.

Hekinian, $R$, and D. Walker, Diversity and spatial zonation of volcanic rocks from the East Pacific Rise near $21^{\circ} \mathrm{N}$, Contrib. Mineral. Petrol., 96, 265, 1987.

Hekinian, R., G. Thompson, and D. Bideau, Axial and off-axial heterogeneity of basaltic rocks from the East Pacific Rise at $12^{\circ} 35^{\prime} \mathrm{N}-12^{\circ} 51^{\prime} \mathrm{N}$ and $11^{\circ} 26^{\prime} \mathrm{N}-11^{\circ} 30^{\prime} \mathrm{N}, J$. Geophys. Res., 94, $17,437-17,463,1989$.

Hofmann, A. W., Chemical differentiation of the Earth: the relationship between mantle, continental crust, and oceanic crust, Earth and Planet. Sci. Lett., 90, 297-314, 1988.

Humphris, S. E., G. Thompson, J.-G. Schilling, and R. H. Kingsley, Petrological and geochemical variations along the Mid-Altantic Ridge between $46^{\circ} \mathrm{S}$ and $32^{\circ} \mathrm{S}$ : Influence of the Tristan de Cunha mantle plume, Geochim. Cosmochim. Acta, 49, 1445-1464, 1985.

IHO/IOC/CHS. GEBCO - General Bathymetric Chart of the Ocean (5th Edition). Intemat'1 Hydrographic Organization/Intergovernmental Oceanographic Commission, Canadian Hydrographic Service, Ottawa, Canada, 74 p., 1984.

Irving, A. J., A review of experimental studies of crystal/liquid trace element partitioning, Geochim. Cosmochim. Acta, 42, 743-770, 1978.

Jackson, H. R., and I. Reid, Crustal refraction and seismic reflection results from FRAM 1, EOS, 61, 276, 1980.

Jakobsson, S. P., J. Jonsson, and F. Shido, Petrology of the western Reykjanes Peninsula, Iceland, J. Petrol., 19, 669-705, 1978.

Jagoutz, E., H. Palme, H. Baddenhausen, K. Blum, M. Cendales, G. Dreibus, S. Spettel, V. Lorenz, and H. Wanke, The abundances of major, minor and trace elements in the Earth's primitive mantle as derived from primitive ultramafic nodules, Proc. Lunar Planet. Sci. Conf., 10th, 2031-2050, 1979.

Jaques, A. L., and D. H. Green, Anhydrous melting of peridotite at 0-15 $\mathrm{kb}$ pressure and genesis of tholeiitic basalts, Contrib. Mineral. Petrol., 73, 287-310, 1980.

Jochum, K. P., A. W. Hofmann, E. Ito, H. M. Seufert, and W. M. White, $K, U$ and $T h$ in mid-ocean ridge basalt glasses and heat production, $\mathrm{K} / \mathrm{U}$ and $\mathrm{K} / \mathrm{Rb}$ in the mantle, Nature, 306, 431-436, 1983.

Johnson, K. T. M., H. J. B. Dick, and N. Shimizu, Melting in the oceanic upper mantle: an ion microprobe study of diopsides in abyssal peridotites, J. Geophys. Res., 95, 2661-2678, 1990. 
Johnson, K. T. M., and H. J. B. Dick, Open system melting and temporal and spatial variation of peridotite and basalt at the Atlantis II Fracture Zone, J. Geophys. Res. 97, 9219-9241, 1992.

Karson, J. A., et al., Along-axis variations in seafloor spreading in the MARK area, Nature, 328, 681-685, 1987.

Karsten, J. L., J. R. Delaney, J. M. Rhodes, and R. A. Liias, Spatial and temporal evolution of magmatic systems beneath the endeavor segment, Juan de Fuca Ridge: tectonic and petrologic constraints, $J$. Geophys. Res., 95, 19235-19256, 1990.

Keleman, P. B., Reaction between ultramafic rock and fractionating basaltic magma I. Phase relations, the origin of calc-alkaline magma series, and the formation of discordant dunite, J. Petrol., 31, 51-98, 1990.

Kinzler, R. J., and T. L. Grove, Primary magmas of mid-ocean ridge basalts. I. Experiments and methods, J. Geophys. Res., in press, $1992 a$.

Kinzler, R. J., and T. L. Grove, Primary magmas of mid-ocean ridge basalts. II. Applications, J. Geophys. Res., in press, $1992 b$.

Klein, E. M., Geochemistry of ocean ridge basalts: Mantle processes revealed by major element, trace element and isotopic variations, Ph.D. Thesis, Columbia University, New York, 1989.

Klein, E. M., and C. H. Langmuir, Global correlations of ocean ridge basalt chemistry with axial depth and crustal thickness, J. Geophys. Res., 92, 8089-8115, 1987.

Klein, E. M., and C. H. Langmuir, Local versus global variations in ocean ridge basalt composition: a reply, J. Geophys. Res., 94 4241-4252, 1989.

Klein, E. M., C. H. Langmuir, and H. Staudigel, Geochemistry of basalts from the Southeast Indian Ridge, $115^{\circ} \mathrm{N}-138^{\circ} \mathrm{E}$, J. Geophys. Res., 96, 2089-2108, 1991.

Klein, E. M., C. H. Langmuir, J. Bender, R. Batiza, and A. Zindler, Nd and $\mathrm{Sr}$ isotopic composition of volcanic glasses from the East Pacific Rise ( $\left.8^{\circ} \mathrm{N}-12^{\circ} \mathrm{N}\right)$, EOS, 67, 1255, 1986.

Kuo, L. C., and R. J. Kirkpatrick, Pre-eruption history of phyric basalts from DSDP Legs 45 and 46: evidence from morphology and zoning patterns in plagioclase, Contrib. Mineral. Petrol. 79, 13-27, 1982 .

Langmuir, C. H., A major element approach to basalts, PhD Thesis, SUNY, Stony Brook, NY, 1980.

Langmuir, C. H., Petrology data base, vol II and III, in East Pacific Rise Data Synthesis Final Report, edited by S. Tighe, Joint Oceanographic Institutes Inc., Washington, D.C., 1988.

Langmuir, C. H, Geochemical consequences of in situ crystallization, Nature, 340, 199-205, 1989.

Langmuir, C. H., Major element systematics of ocean island basalts, Caltech Plume Symposium, Pasadena, CA, 1991.

Langmuir, C. H., and J. F. Bender, The geochemistry of oceanic basalts in the vicinity of transform faults: Observations and implications, Earth Planet. Sci. Lett., 69, 107-127, 1984.

Langmuir, C. H., and G. N. Hanson, An evaluation of major element heterogeneity in the mantle sources of basalts, Phil. Trans. $R$. Soc. Lond., 297, 383-407, 1980.

Langmuir, C. H., and G. N. Hanson, Calculating mineral-melt equilibria with stoichiometry, mass balance, and single-component distribution coefficients, in Thermodynamics of Minerals and Melts Advances in Physical Geochemistry, Vol. 1, edited by R. C. Newton, A. Navrotsky, and B. J. Wood, pp. 247-271, SpringerVerlag, New York, 1981 .

Langmuir, C. H., J. F. Bender, and R. Batiza, Petrological and tectonic segmentation of the East Pacific Rise, $5^{\circ} 30^{\prime}-14^{\circ} 30^{\prime} \mathrm{N}$, Nature, 332, 422-429, 1986.

Langmuir, C. H., J. F. Bender, A. E. Bence, G. N. Hanson, and S. R.Taylor, Petrogenesis of basalts from the FAMOUS area: MidAtlantic Ridge, Earth Planet. Sci. Lett., 36, 133-156, 1977.

LaTourrett, T. Z., and D. S. Burnett, Experimental determination of U and Th partitioning between clinopyroxene and natural basaltic silicate liquid, Earth Planet. Sci. Lett., 100, 227-244, 1992.

LeDouaran, S. L., and J. Francheteau, Axial depth anomalies from 10 to $50^{\circ}$ north along the Mid-Atlantic Ridge: correlation with other mantle properties, Earth Planet. Sci. Lett., 54, 29-47, 1981.
LeRoex, A. P., H. J. B Dick, and R. L. Fisher, Petrology and Geochemistry of MORB from $25^{\circ} \mathrm{E}$ to $46^{\circ} \mathrm{E}$ along the Southwest Indian Ridge: Evidence for contrasting styles of mantle enrichment, J. Petrol., 30, 947-986, 1989.

Lin, J., G. M. Purdy, H. Schouten, J.-C. Sempere, and C. Zervas, Evidence from gravity data for focused magmatic accretion along the Mid-Atlantic Ridge, Nature, 344, 627-632, 1990.

Longhi, J., Comparative liquidus equilibria of hypersthene-normative basalts at low pressure, Am. Mineral., 76, 785-800, 1991.

Longhi, J., Liquidus equilibria and solid solution in the system $\mathrm{CaAl}_{2} \mathrm{Si}_{2} \mathrm{O}_{8}-\mathrm{Mg}_{2} \mathrm{SiO}_{4}-\mathrm{CaSiO}_{3}-\mathrm{SiO}_{2}$ at low pressure, Am. J. Sci., 287, 265-331, 1987.

Maaloe, S., and K. Aoki, The major element composition of the upper mantle estimated from the composition of lherzolites, Contrib. Mineral. Petrol., 63, 161-173, 1977.

Macdonald, K. C., P. J. Fox, L. J. Perram, M. F. Eisen, R. M. Haymon, S. P. Miller, S. M. Carbotte, M.-H. Cormier, and A. N. Shor, A new view of the mid-ocean ridge from the behavior of ridge-axis discontinuities, Nature, 335, 217-225, 1988.

McKenzie, D., The generation and compaction of partially molten rock, J. Petrol., 25, 713-765, 1984.

McKenzie, D., ${ }^{230} \mathrm{Th}-{ }^{238} \mathrm{U}$ disequilibrium and the melting processes beneath ridge axes, Earth Planet. Sci. Lett., 72, 149-157, 1985 a.

McKenzie, D., The extraction of magma from the crust and mantle, Earth Planet. Sci. Lett., 74, 81-91, $1985 b$.

McKenzie, D., and M. J. Bickle, The volume and composition of melt generated by extension of the lithosphere, J. Petrol., 29, 625-679, 1988 .

McKenzie, D., and R. K. O'Nions, Partial melt distributions from inversion of rare earth element concentrations, J. Petrol., 32, 1021 $1091,1991$.

Melson, W. G., and T. O'Hearn, Basaltic glass erupted along the MidAtlantic Ridge between $0-37^{\circ} \mathrm{N}$ : Relationships between composition and latitude, in Deep Sea Drilling Results in the Atlantic Ocean: Ocean Crust, Maurice Ewing Ser., vol. 2, edited by M. Talwani, C. G. Harrison, and D. E. Hayes, pp. 249-261, AGU, Washington, D.C., 1979.

Melson, W. G., G. R. Byerly, J. A. Nelson, T. O'Heam, T. L. Wright, and T. Vallier, A catalogue of the major element chemistry of abyssal volcanic glasses, Smithsonian Contrib. Earth Sci., 19, 31-60, 1977.

Michael, P. J., The concentration, behavior and storage of $\mathrm{H}_{2} \mathrm{O}$ in the suboceanic upper mantle: Implications for mantle metasomatism, Geochim. Cosmochim. Acta, 52, 555-566, 1988.

Michael, P. J., and E. Bonatti, Peridotite composition from the North Atlantic: Regional and tectonic variations and implications for partial melting, Earth Planet. Sci. Lett., 73, 91-104, 1985.

Michael, P. J., R. L. Chase, and J. F. Allen, Petrologic and geologic variations along the southem Explorer Ridge, northeast Pacific Ocean, J. Geophys. Res., 94, 13,895-13,918, 1989.

Miyashiro, A., F. Shido, and M. Ewing, Diversity and origin of abyssal tholeiite from the Mid-Atlantic Ridge near $24^{\circ}$ and $30^{\circ} \mathrm{N}$, Contrib. Mineral. Petrol., 23, 38-52, 1969.

Morel, J. M., and R. Hekinian, Compositional variations of volcanics along segments of recent spreading ridges, Contrib. Mineral. Petrol., 72, 425-436, 1980.

Muir, I. D., C. E. Tilley, and J. H. Scoon, Basalts from the northern part of the rift zone of the Mid-Atlantic Ridge, J. Petrol., 5, 269$301,1964$.

Mysen, B. O., and I. Kushiro, Compositional variations of coexisting phases with degree of melting of peridotite in the upper mantle, Am. Mineral., 62, 843-856, 1977.

Natland, J. G., and W. G. Melson, Comparisons of basaltic glasses from the East Pacific Rise and Siqueiros Fracture Zone, near $9^{\circ} \mathrm{N}$, Init. Reports, DSDP, 54, 705-23, 1980.

Natland, J. H., Partial melting of a lithologically heterogeneous mantle: inferences from crystallization histories of magnesian abyssal tholeiites from the Siqueiros Fracture Zone, in Magmatism in the Ocean Basins, edited by A. D. Saunders and M. J. Norry, pp. 41-70, Geol. Soc. Sp. Publ. No. 42, 1989. 
Nelson, D. R., A. R. Chivas, B. W. Chappell, and M. T. McCulloch, Geochemical and isotopic systematics in carbonatites and implications for the evloution of ocean-island sources, Geochim. Cosmochim. Acta, 52, 1-17, 1988.

Neumann, E.-R., and J.-G. Schilling, Petrology of basalts from the Mohns-Knipovich Ridge: The Norwegian-Greenland Sea, Contrib. Mineral. Petrol., 85, 209-223, 1984.

Nielsen, R. L., EQUIL: A program for the modeling of low pressure differentiation processes in natural mafic magma bodies, Computers and Geosci., 11, 531-546, 1985.

Nielsen, R. L., Phase equilibria constraints on liquid lines of descent generated by paired assimilation and fractional crystallization: trace elements and $\mathrm{Sr}$ and $\mathrm{Nd}$ isotopes, J. Geophys. Res., 94, 787-794, 1989.

Nielsen, R. L., The theory and application of a model of open magma system processes, in Modern Methods of Petrology: Reviews in Mineralogy, 24, edited by J. Nicholls and J. K. Russel, pp. 65-106, Mineralogical Society of America, 1990.

Nielsen, R. L., and M. J. Drake, Pyroxene-melt equilibria, Geochim. Cosmochim. Acta, 43, 1259-1272, 1979.

Nielsen, R. L., and M. A. Dungan, Low pressure mineral-melt equilibria in natural anhydrous mafic systems, Contrib. Mineral. Petrol., 84, 310-326, 1983

Niu, Y., and R. Batiza, An empirical method for calculating melt compositions produced beneath mid-ocean ridges: Application for axis and off-axis (seamounts) melting, J. Geophys. Res., 96, 21,753-21,777, 1991 .

O'Hara, M. J. and R. E. Matthews, Geochemical evolution in an advancing periodically replenished, periodically tapped, continuously fractionated magma chamber, J. Geol. Soc. London, $138,237-277,1981$

O'Hara, M. J., Are any ocean floor basalts primary magma?, Nature, $220,683-686,1968 a$.

$\mathrm{O}$ 'Hara, M. J., The bearing of phase equilibria studies in synthetic and natural systems on the origin and evolution of basic and ultrabasic rocks, Earth Sci. Rev., 4, 69-133, 1968 b.

O'Hara, M. J., Geochemical evolution during fractional crystallization of a periodically refilled magma chamber, Nature, 266, 503-507, 1977.

O'Hara, M. J., Importance of the "shape" of the melting regime during partial melting of the mantle, Nature, 314, 58-62, 1985.

O'Hara, M. J., Primary magmas and the origin of basalts, Scot. J. Geol., 1, 19-40, 1965.

Onuma, N., H. Higuchi, H. Wakita, and H. Nagasawa, Trace element partition between two pyroxenes and the host lava, Earth Planet. Sci. Lett., 5, 47-51, 1968.

Oxburgh, E. R., Heat flow and magma genesis, in Physics of Magmatic Processes, edited by R. B. Hargraves, pp. 161-199, Princeton University Press, Princeton, NJ, 1980.

Perfit, M. R., D. J. Fornari, A. Malahoff, and R. W. Embley, Geochemical studies of abyssal lavas recovered by DSRV Alvin from Eastem Galapagos Rift, Inca Transform, and Ecuador Rift 3. Trace element abundances and petrogenesis, J. Geophys. Res., 88, $10,551-10,572,1983$

Philpotts, J. A., The law of constant rejection, Geochim. Cosmochim. Acta, 42, 909-920, 1978

Phipps Morgan, J., Melt migration beneath mid-ocean spreading centers, Geophys. Res. Lett., 14, 1238-1241, 1987.

Plank, T., and C. H. Langmuir, Effects of the melting regime on the composition of the oceanic crust, J. Geophys. Res., 97, 19,749$19770,1992$.

Presnall, D. C., The geometric analysis of partial fusion, Am. J. Sci., 267, 1178-1194, 1969.

Presnall, D. C., J. R. Dixon, T. H. O'Donnell, and S. A. Dixon, Generation of mid-ocean ridge tholeiites, J. Petrol., 20, 3-35, 1979.

Presnall, D. C. and J. D. Hoover, Composition and depth of origin of primary mid-ocean ridge basalts, Contrib. Mineral. Petrol., 87, 170178, 1984.

Price, R. C., A. K. Kennedy, M. Riggs-Sneeringer, and F.A. Frey, Geochemistry of basalts from the Indian Ocean triple junction:
Implications for the generation and evolution of Indian Ocean ridge basalts, Earth Planet. Sci. Lett., 78, 379-396, 1986.

Prinzhofer, A., E. Lewin, and C. J. Allégre, Stochastic melting of the marble cake mantle: evidence from local study of the East Pacific Rise at $12^{\circ} 50$ N, Earth Planet. Science Lett., 92, 189-106, 1989.

Reid, I., and H. R. Jackson, Oceanic spreading rate and crustal thickness, Marine Geophys. Res., 5, 165-172, 1981.

Reinitz, I., and K. K. Turekian, ${ }^{230} \mathrm{Th} /{ }^{38} \mathrm{U}$ and ${ }^{226} \mathrm{Ra} /{ }^{30} \mathrm{Th}$ fractionation in young basaltic glasses from the East Pacific Rise, Earth and Planet. Sci. Lett. 94, 199-207, 1989.

Reisberg, L. C., The isotopic and geochemical systematics of the Ronda Ultramafic Complex of southem Spain, PhD. Thesis, Columbia University, N. Y., N. Y., 1988.

Reisberg, L., and A. Zindler, Extreme isotopic variations in the upper mantle: evidence from Ronda, Earth Planet. Sci. Lett., 81, 29-45, $1986 / 87$.

Reynolds, J. R., C. H. Langmuir, J. F. Bender, K. A. Kastens, and W. B. F. Ryan, Spatial and temporal variability in the geochemistry of basalts from the East Pacific Rise, Nature, 359, 493-499, 1992.

Reynolds, J. R., and C. H. Langmuir, Petrological systematics of the spreading cell south of the Kane Transform: implications for models of ocean crust formation, J. Geophys. Res., in revision, 1992.

Richter, F. M., Simple models for trace element fractionation during melt segregation, Earth Planet. Sci. Lett., 77, 333-344, 1986.

Roeder, P. L., Activity of iron and olivine solubility in basaltic melts, Earth Planet. Sci. Lett., 23, 397-410, 1974.

Roeder, P. L., and R. F. Emslie, Olivine-liquid equilibrium, Contrib. Mineral. Petrol., 29, 275-289, 1970.

Rubin, K. H., and J. D. Macdougall, ${ }^{226} \mathrm{Ra}$ excesses in mid-ocean ridge basalts and mantle melting, Nature, 336, 158-161, 1988.

Rubin, K. H., and J. D. Macdougall, Dating of neovolcanic MORB using $\left({ }^{226} \mathrm{Ra} /{ }^{230} \mathrm{Th}\right)$ disequilibrium, Earth and Planet. Sci. Lett., 101 , 313-322, 1990.

Sack, R. O., D. Walker, and I. S. E. Carmichael, Experimental petrology of alkalic lavas: constraints on cotectics of multiple saturation in natural basic liquids, Contrib. Mineral. Petrol., 96, 1$23,1987$.

Salters, V. J. M., and N. Shimizu, World-wide occurrence of HFSEdepleted mantle, Geochim. Cosmochim. Acta, 52, 2177-2182, 1988.

Salters, V. J. M., and S. R. Hart, The hafnium paradox and the role of garnet in the source of mid-ocean ridge basalts, Nature, 342, 420 $422,1989$.

Sarda, P., and D. Graham, Mid-ocean ridge popping rocks: implication for degassing at ridges, Earth Planet. Sci. Lett., 97, 268, 1990.

Schilling, J-G., Rare earth fractionation in Hawaii volcanic rocks, Ph.D. Thesis, Mass. Inst. Technology, 1966.

Schilling, J-G., Sea-floor evolution: rare-earth evidence, Phil. Trans. Roy. Soc. London, A268, 663-706, 1971.

Schilling, J-G., Iceland mantle plume: Geochemical study of Reykjanes Ridge, Nature, 242, 565-571, 1973.

Schilling, J-G., Azores mantle blob: Rare earth evidence, Earth Planet. Sci. Lett., 25, 103-115, 1975.

Schilling, J-G., Upper mantle heterogeneities and dynamics, Nature $314,62-67,1985$.

Schilling, J-G., Geochemical and isotopic variation along the MidAtlantic Ridge axis from $79^{\circ} \mathrm{N}$ to $0^{\circ} \mathrm{N}$, in The Geology of North America, Vol. M, The Western North Atlantic Region, edited by $P$. R. Vogt and B. E. Tucholke, Geological Society of America, 1986.

Schilling, J-G., and H. Sigurdsson, Thermal minima along the axis of Mid-Atlantic Ridge, Nature, 282, 370-5, 1979.

Schilling, J-G, R. N. Anderson, and P. Vogt, Rare earth, Fe and Ti variations along the Galapagos spreading centre, and their relationship to the Galapagos mantle plume, Nature, 261, 108-113, 1976.

Schilling, J-G., M. B. Bergeron, and R. Evans, Halogens in the mantle beneath the N. Atlantic, Phil. Trans. Royal Soc. London, A297, $147-178,1980$.

Schilling, J-G., R. H. Kingsley and J. D. Devine, Galapagos hot spotspreading center system 1 . Spatial petrological and geochemical 
variations $\left(83^{\circ} \mathrm{W}-101^{\circ} \mathrm{W}\right), J$. Geophys. Res., 87, 5593-5610, 1982.

Schilling, J.-G., H. Sigurdsson, A. N. Davis, and R. N. Hey, Easter microplate evolution, Nature, 317, 325-331, 1985.

Schilling, J-G, M. Zajak, R. Evans, T. Johnson, W. White, J. D. Devine and R. Kingsley, Petrologic and geochemical variations along the Mid-Atlantic Ridge from $29^{\circ} \mathrm{N}$ to $73^{\circ} \mathrm{N}$, Am. J. Sci., 283 , 510-586, 1983.

Schouten, H., K. D. Klitgord, and J. A. Whitehead, Segmentation of mid-ocean ridges, Nature, 317, 225-229, 1985.

Scott, D. R., and D. J. Stevenson, A self consistent model of melting, magma migration and buoyancy driven circulation beneath midocean ridges, J. Geophys. Res., 94, 2973-2988, 1989.

Shaw, D. M., Trace element fractionation during anatexis, Geochim. Cosmochim. Acta, 34, 237-243, 1970.

Shibata, T., G. Thompson, and F. A. Frey, Tholeiitic and alkali basalts from the mid-Atlantic Ridge at $43^{\circ} \mathrm{N}$, Contrib. Mineral. Petrol., 70 , 127-141, 1979.

Shirey, S. B., J. F. Bender, and C. H. Langmuir, Three-component isotopic heterogeneity near the Oceanographer transform, MidAtlantic Ridge, Nature, 325, 217-223, 1987.

Sigurdsson, H., First-order major element variation in basaltic glasses from the Mid-Atlantic Ridge: $29^{\circ} \mathrm{N}$ to $73^{\circ} \mathrm{N}, J$. Geophys. Res., 86, 9483-9502, 1981.

Sinton, J. M. and R. S. Detrick, Mid-ocean ridge magma chambers, $J$. Geophys, Res., 97, 197-216, 1992.

Sinton, J. M., D. S. Wilson, D. M. Christie, R. N. Hey, and J. R. Delaney, Petrologic consequences of rift propagation on oceanic spreading centers, Earth Planet. Sci. Lett., 62, 193-207, 1983.

Sinton, J. M., S. M. Smaglik, and J. J. Mahoney, Magmatic processes at superfast spreading mid-ocean ridges: glass compositional variations along the East Pacific Rise $13^{\circ}-23^{\circ} \mathrm{S}, J$. Geophys. Res. $96,6133-6155,1991$.

Sleep, N. H., Segregation of magma from a mostly crystalline mush, Geol. Soc. Am. Bull., 85, 1225-1232, 1974.

Small, C., and D. T. Sandwell, An abrupt change in ridge axis gravity with spreading rate, J. Geophys. Res., 94, 17383-17392, 1989.

Sparks, R. S. J., In situ differentiation, Nature, 340, 187, 1989.

Sparks, R. S. J., H. E. Huppert, and J. S. Tumer, Fluid dynamics of involved magma chambers, Phil. Trans. R. Soc., London, A310 511-534, 1984.

Spiegelman, M., and D. McKenzie, Simple 2-D models for melt extraction at mid-ocean ridges and island arcs, Earth Planet. Sci. Lett., 83, 137-152, 1987.

Stakes, D. S., J. W. Shervais, and C. A. Hopson, The volcanic-tectonic cycle of the FAMOUS and AMAR Valleys, Mid-Atlantic Ridge $\left(36^{\circ} 47^{\circ} \mathrm{N}\right)$ : Evidence from basalt glass and phenocryst compositional variations for a steady state magma chamber beneath the valley midsections, AMAR 3, J. Geophys. Res., 89, 6995-7028, 1984.

Stolper, E., A phase diagram for mid-ocean ridge basalts: Preliminary results and implications for petrogenesis, Contrib. Mineral. Petrol. 74, 13-27, 1980.

Stroup, J. B., and P. J. Fox, Geologic investigations in the Cayman Trough: Evidence for thin oceanic crust along Mid-Cayman Rise, $J$. Geol., 89, 395-420, 1981.

Sun, S.-S., Lead isotopic study of young volcanic rocks from midocean ridges, ocean islands and island arcs, Phil. Trans. R. Soc. London, A297, 409-446, 1980.

Sun, S.-S. and G. N. Hanson, Origin of Ross Island basanitoids and limitations upon the heterogeneity of mantle sources for alkali basalts and nephelinites, Contrib. Mineral. Petrol. 52, 77-106, 1975.

Sun, S.-S., R. W. Nesbitt, and A. Y. Sharaskin, Geochemical characteristics of mid-ocean ridge basalts, Earth Planet. Lett., 44, 119-138, 1979.

Sun, S.-S., M. Tatsumoto, and J.-G. Schilling, Mantle plume mixing along the Reykjanes Ridge axis: Lead isotope evidence, Science, 190, 143-147, 1975.
Takahashi, E., and I. Kushiro, Melting of a dry peridotite at high pressures and basalt magma genesis, Am. Mineral., 68, 859-879, 1983.

Takahashi, E., Melting of a dry peridotite KLB-1 up to $14 \mathrm{GPa}$ : implication on the origin of peridotitic upper mantle, $J$. Geophys. Res., 91, 9367-9382, 1986.

Thompson, G., W. B. Bryan, and W .G. Melson, Geological and geophysical investigation of the Mid-Cayman Rise spreading center: Geochemical variation and petrogenesis of basalt glasses, $J$. Geol., 88, 41-55, 1980.

Thompson, G., W. B. Bryan, R. Ballard, K. Hamuro, and W. G. Melson, Axial processes along a segment of the East Pacific Rise, $10^{\circ}-12^{\circ} \mathrm{N}$, Nature, $318,429-433,1985$.

Thompson, R. N., M. A. Morrison, A. P. Dickin, and G. L. Hendry, Continental flood basalts Arachnids rule OK?, in Continental Flood Basalts and Mantle Xenoliths, edited by C. J. Hawkesworh and M. J. Norry, pp. 158-185, Shiva, Cheshire, 1983.

Tormey, D. R., T. L. Grove, and W. B. Bryan, Experimental petrology of normal MORB near the Kane Fracture Zone, $22^{\circ}-25^{\circ} \mathrm{N}$, mid. Atlantic ridge, Contrib. Mineral. Petrol., 94, 121-139, 1987.

Verma, S. P., and J.-G. Schilling, Galapagos hot spot center system, $2,{ }^{87} \mathrm{Sr} /{ }^{86} \mathrm{Sr}$ and large ion lithophile element variations $\left(85^{\circ} \mathrm{W}\right.$ $101^{\circ}$ W), J. Geophys. Res., 87, 10,838-10,856, 1982.

Vogt, P. R., Portrait of a plate boundary: the Mid-Atlantic Ridge axis from the equator to Siberia, in The Western North Atlantic Region, The Geology of North America, vol. M, edited by P. R. Vogt and B. E. Tucholke, plate 8A, Geol. Soc. Am., Boulder, CO., 1986.

Watson, S., and D. McKenzie, Melt generation by plumes: A study of Hawaiian volcanism, J. Petrol., 32, 501-537, 1991.

Walker, D., T. Shibata, and S. E. DeLong, Abyssal tholeiites from the Oceanographer Fraction Zone. II. Phase equilibria and mixing, Contrib. Mineral. Petrol., 70, 111-125, 1979.

Weaver, J., and C. Langmuir, Calculation of phase equilibrium in mineral-melt systems, Computers and Geosciences, 16, 1-19, 1990.

White, W. M., and W. B. Bryan, Sr-isotope, K, Rb, Cs, Sr, Ba, and rare-earth geochemistry of basalts from the FAMOUS area, Geol. Soc. Am. Bull., 88, 571-576, 1977.

White, W. M., A. W. Hofmann, and H. Puchelt, Isotope geochemistry of Pacific mid-ocean ridge basalt, J. Geophys. Res., 92, 4881-4893, 1987.

Whitehead, J. A., H. J. A. Dick, and H. Schouten, A mechanism for magmatic accretion under spreading centres, Nature, 312, 146-148, 1984.

Williams, R. W., and J. B. Gill, Effects of partial melting on the uranium decay series, Geochim. Cosmochim. Acta, 53, 1607-1919, 1989.

Wood, D. A., A variably veined suboceanic upper mantle -- Genetic significance for mid-ocean ridge basalts from geochemical evidence, Geology, 7, 499-503, 1979.

Wyllie, P. J., Discussion of recent papers on carbonated peridotite, bearing on mantle metasomatism and magmatism, Earth Planet. Sci. Lett., 82, 391-397, 1987.

Yoder, H. S., Generation of Basaltic Magma, pp. 209, National Academy of Sciences, Washington, 1976.

Zindler, A., and S. Har, Chemical Geodynamics, Annu. Rev. Earth Planet. Sci., 14, 493-571, 1986.

Zindler, A., H. Staudigel, and R. Batiza, Isotope and trace element geochemistry of young Pacific seamounts: Implications for the scale of upper mantle heterogeneity, Earth Planet. Sci. Lett., 70, 175-195, 1984 .

C. Langmuir and T. Plank, Lamont-Doherty Geological Observatory of Columbia University, Palisades, NY 10964

E. Klein, Department of Geology, Duke University, Durham, NC 27706 